STRUCTURING UNSTRUCTURED CLINICAL NARRATIVES IN OPENMRS WITH MEDICAL CONCEPT EXTRACTION

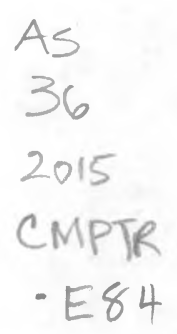

A thesis submitted to the faculty of

San Francisco State University

In partial fulfillment of

the requirements for

the Degree

Master of Science

$\ln$

Computer Science

by

Ryan Michael Eshleman

San Francisco, California

August 2015 
Copyright by

Ryan Michael Eshleman

2015 


\section{CERTIFICATION OF APPROVAL}

I certify that I have read "Structuring Unstructured Clinical Narratives in OpenMRS with Medical Concept Extraction" by Ryan Eshelmen, and that in my opinion this work meets the criteria for approving a project submitted in partial fulfillment of the requirements for the degree: Master of Science of Computer Science at San Francisco State University.

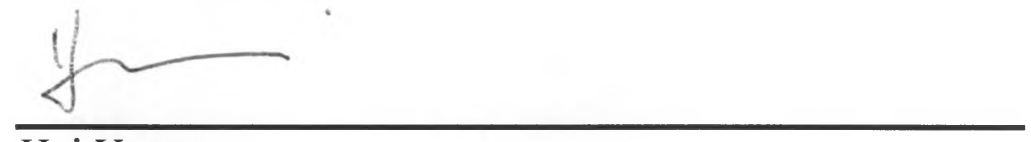

Hui Yang,

Associate Professor of Computer Science

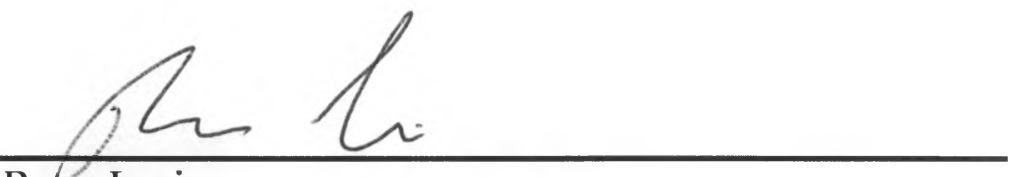

Berry Levine,

Professor of Computer Science

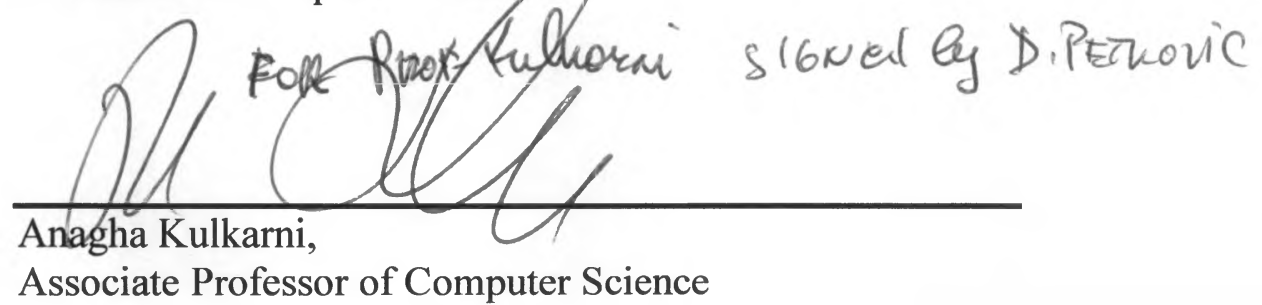




\title{
Structuring Unstructured Clinical Narratives in OpenMRS with Medical Concept Extraction
}

\author{
Ryan Eshlemen \\ San Francisco State University \\ 2015
}

We have developed an extension to the open source Electronic Medical Record System OpenMRS that leverages Named Entity Recognition (NER) to deliver concise, semantic-type driven, interactive summaries of clinical notes. To that end, we performed an extensive empirical evaluation of four NER systems using textual clinical narratives and full biomedical journal articles. The four NER systems under evaluation are the National Library of Medicine's MetaMap, Apache cTAKES, University of Michigan's MGrep, and Arizona State University's BANNER. We studied several ensemble approaches built upon the above four NER systems to exploit their collaborative strengths. Evaluations are performed on the hand annotated patient discharge summaries from the Informatics for Integrating Biology and the Bedside group (I2B2). We also evaluate these NER systems using the CRAFT dataset.

Based on the evaluation results, we have developed a BANNER-based module for OpenMRS to recognize semantic concepts including problems, tests, and treatments in clinical notes. This module works with OpenMRS version 2.x. An API is also developed that allows future developers to build on top of the NER capabilities. We have also developed a companion web application to train the BANNER model based on data from the OpenMRS database which allows the user to iteratively tune the NER model for optimal performance.

I certify that the Abstract is a correct representation of the content of this thesis.
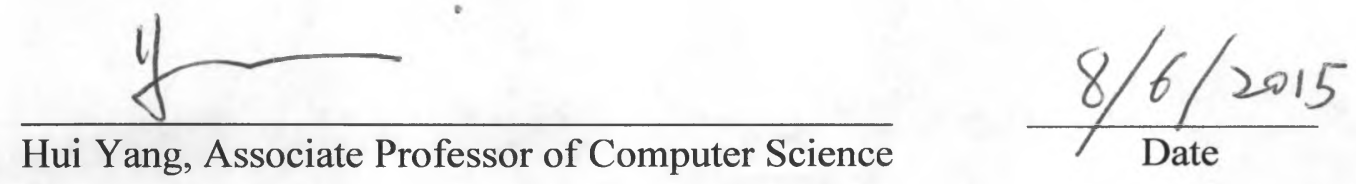


\section{ACKNOWLEDGEMENTS}

I am indebted to my advising committee for all of the opportunities and guidance that they have provide during my time at SFSU. Without their support and invaluable insight this project would not have been possible and my graduate studies would not have been

nearly as rich and meaningful. Thanks to: Professor Hui Yang for, among many many things, introducing me to Natural Language Processing and its applications; Professor Barry Levine for the opportunity to put my passions to work in the community by connecting me with OpenMRS and for leading by example; Professor Anagha Kulkarni, whose instruction laid a lot of the groundwork for my understanding of text processing. Thank you to my friends and family, especially to my Mother who was willing to bug her medical colleagues with questions on our behalf. To everyone, thank you. 
TABLE OF CONTENTS

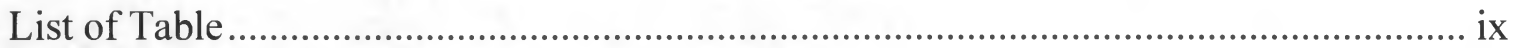

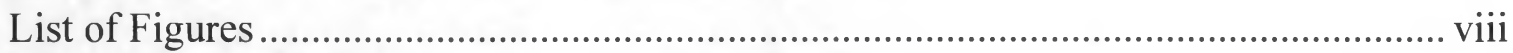

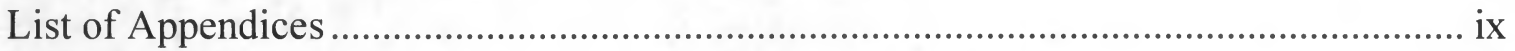

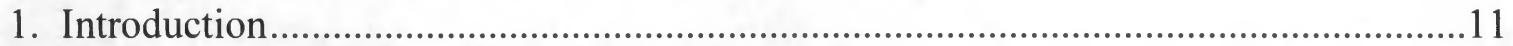

2. EMRS: Background and Related Work .......................................................... 18

2.1 Open Source EMRs ................................................................................. 18

2.2 Applications of NLP in Point of Care Tools..............................................23

3. Named Entity Recognition Systems ..................................................................26

3.1 Apache Clinical Text Analysis and Knowledge Extraction System.............27

3.2 MetaMap, The National Library of Medicine ............................................34

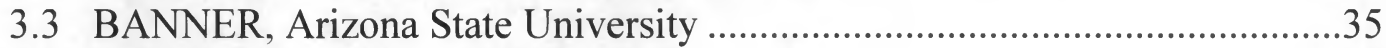

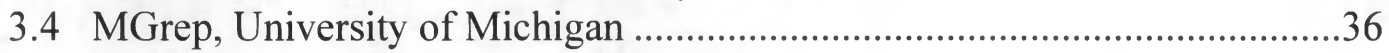

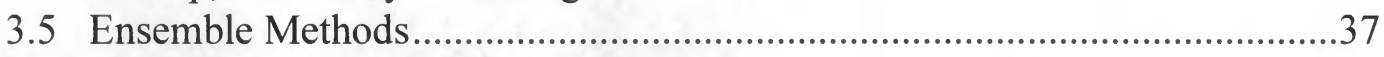

4. Named Entity Recognition Systems Evaluations ...................................................39

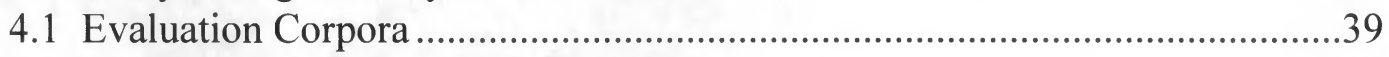

4.2 Evaluation Criteria and Metrics ...............................................................41

4.3 Technical Challenges in Evaluating Multiple Systems ................................42

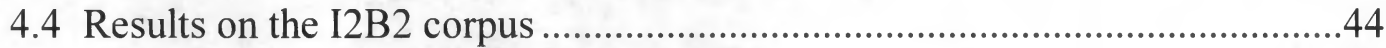

4.4.1 Results for Individual Systems .............................................44

4.4.2 Results for Ensemble Systems ..................................................47

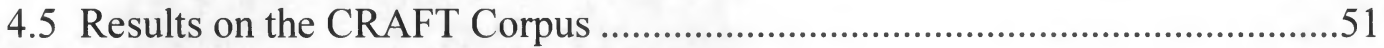

4.6 BANNER Learning Curve and Training Time .........................................53

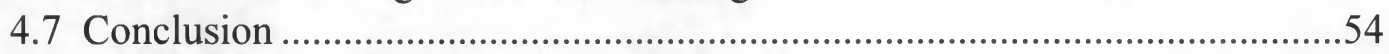

5. Implementation of Clinical Notes Module .........................................................56

5.1 OpenMRS Visit Notes Analysis Module..................................................56

5.1.1 Training the Default BANNER Model ..........................................57

5.1.2 Module Data Model ...................................................................60

5.1.3 NER Algorithm and Implementation........................................63

5.1.4 User Interface....................................................................66

5.2 Visit Notes Analysis and Trainer Application .........................................69

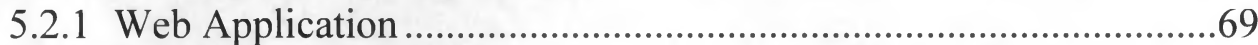

5.2.2 Example Use Case ...................................................................73

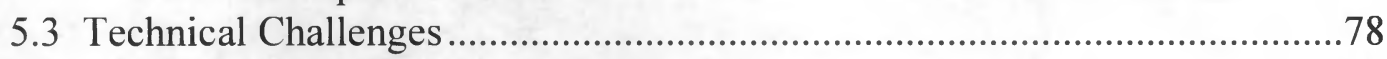

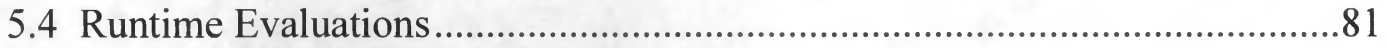

5.5 Feedback from the Community ….................................................... 83

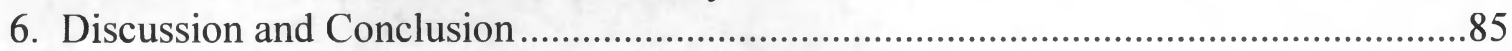




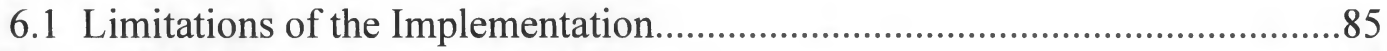

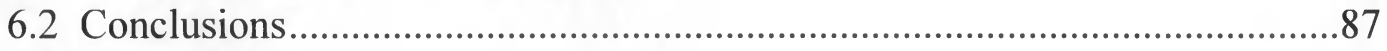

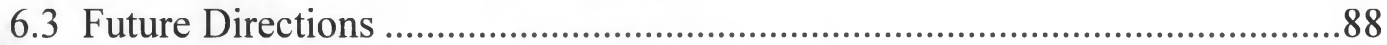

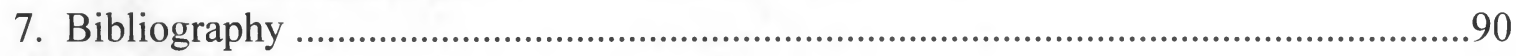

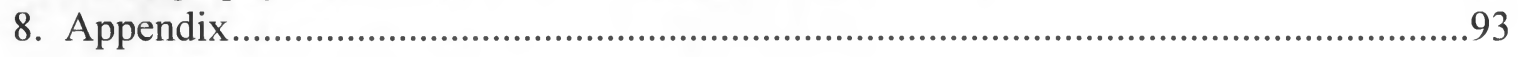




\section{LIST OF TABLES}

Table

Page

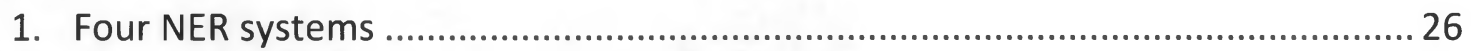

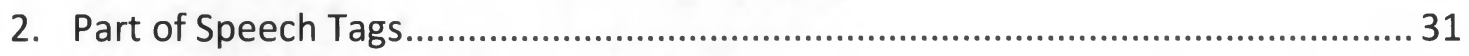

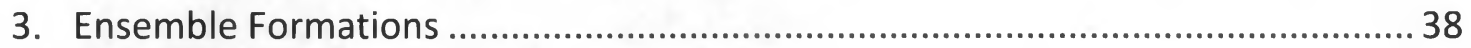

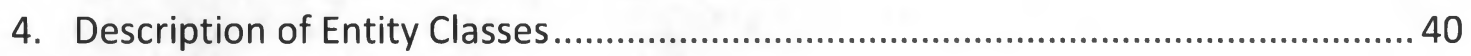

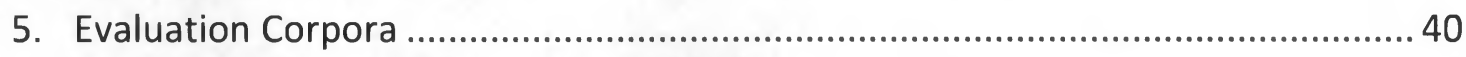

6. Results, No Type Matching, Exact Match...................................................... 44

7. Results, No Type Matching, Single Boundary ................................................. 44

8. Results, No Type Matching, Any Overlap....................................................... 45

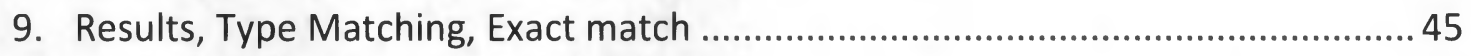

10. Results, Type Matching, Single Boundary ................................................ 45

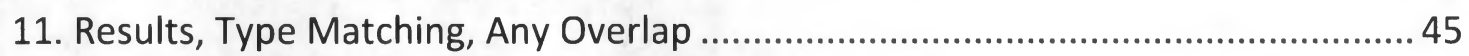

12. Ensemble Results, No Type Matching, Exact Match ......................................... 49

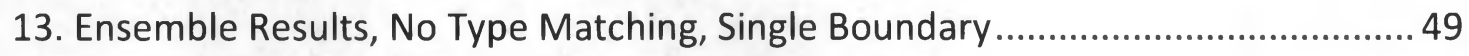

14. Ensemble Results, No Type Matching, Any Overlap ........................................ 49

15. Ensemble Results, Type Matching, Exact match............................................... 50

16. Ensemble Results, Type Matching, Single Boundary ....................................... 50

17. Ensemble Results, Type Matching, Any Overlap............................................... 50

18. OpenMRS Concept Class to Entity Type Mappings.......................................... 63 
Figures

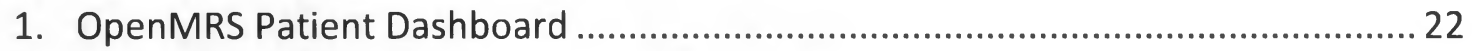

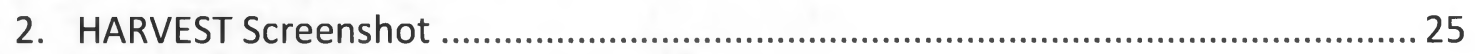

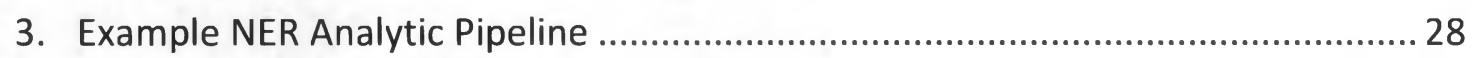

4. Evaluation Breakdown by Type ................................................................. 46

5. Evaluation Results on CRAFT Data Set ....................................................... 52

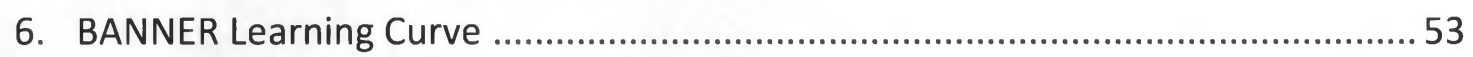

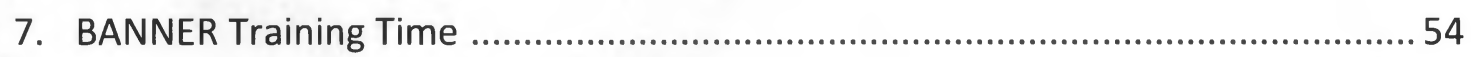

8. Procedure for Training a BANNER model ........................................................5

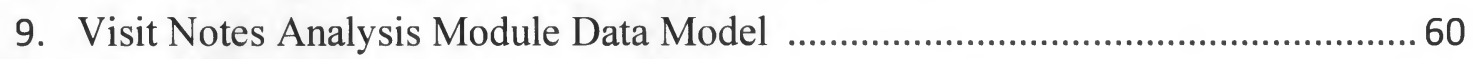

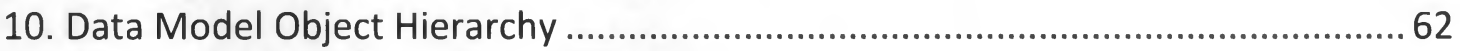

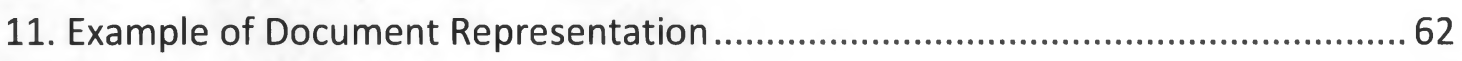

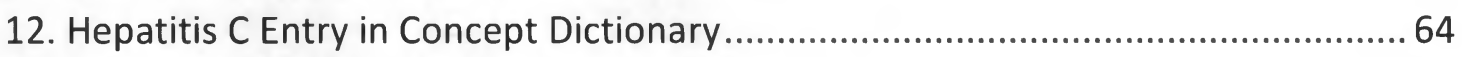

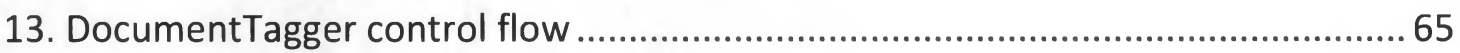

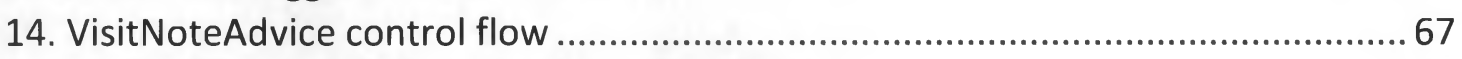

15. Screenshot from Visit Notes Analysis module .................................................6 68

16. Data Flow between Visit Notes Analysis module and Companion Application ..... 70

17. Visit Note Annotator and Trainer screenshot ................................................ 71

18. Visit Note Annotator and Trainer architecture.................................................. 73

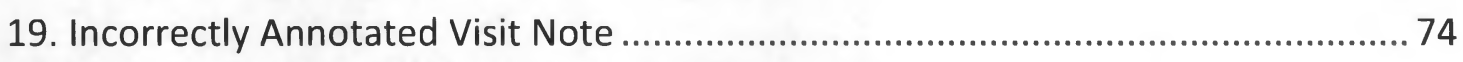

20. Visit Notes loaded into Annotator application .............................................. 76

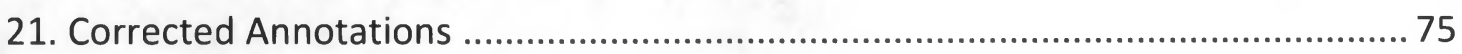

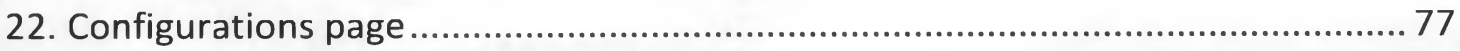

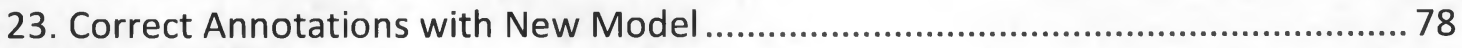

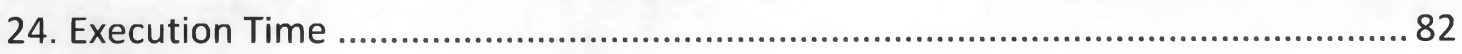

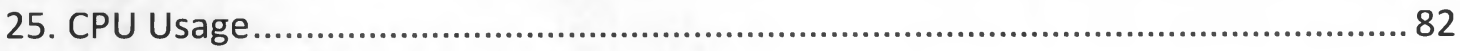

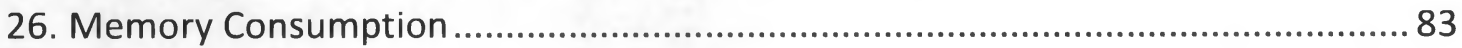




\section{LIST OF APPENDICES}

\section{Appendix}

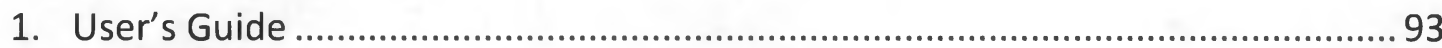

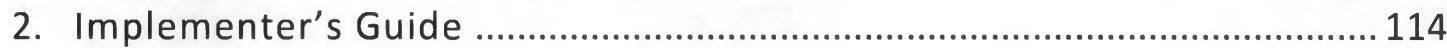

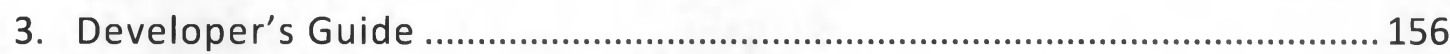

4. Review of Graphical Models for Named Entity Recognition .................... 182 


\section{Chapter 1}

\section{Introduction}

In this thesis project we make an effort to bridge the gap between the current research in the field of Natural Language Processing of bio-medical texts and the analysis of textual data in Electronic Medical Record systems. Recent years have seen considerable progress in the processing of bio-medical texts and the end-goal of much of the research is to improve the overall quality of patient care and patient outcomes. However, applications of this research have not kept pace and clinicians at the point of care with patients often are not seeing the benefits of this research. With this in mind, we developed a module for the Open Source Medical Record System OpenMRS that leverages current research in NLP to offer better cognitive support for clinicians while they review textual patient information.

In today's fast paced clinical setting, in order to achieve satisfying patient outcomes, healthcare providers must equip themselves to efficiently and effectively access and process patient's records. The ability of a health care provider to properly care for the patient depends heavily on their understanding of the patient's current and past medical history. While the advent of the Electronic Medical Record (EMR) has dramatically improved the provider's access to patient information, the EMR often imposes constraints 
on the structure that the patient data can take and does not provide strong support for unstructured data such as text from clinical notes.

One study found that unstructured data accounts for 80 percent of the patient data contained in a medical organization [1]. This means that 80 percent of the information that a care provider needs in order to provide proper care is not in the format that EMRs are designed to support. A study conducted in [3] identified patient notes as a "primary source of information" when reviewing patient history. This asymmetry between the proportion of data for which the EMR is designed (encoded data) and the format of actual patient data (free text) can be very problematic. At best, it can lead to an increase in the time a clinician spends reviewing a patient's records because the relevant data points are mixed with the irrelevant data points. This decrease in efficiency can in turn lead to longer wait times for patients, decreased daily patient throughput, and increased cost for both the patient and the practice. At worst, the data asymmetry can lead to the provider overlooking important data due to either time constraints or information overload [2][49][50]. If the overlooked data happens to be a drug allergy or current drug regimen, the oversight could potentially have severe consequences. Because of this, any improvement in the EMR's ability to process and present unstructured information to the care provider has the potential to improve patient care.

A first step in extracting information from unstructured clinical notes is identifying the location within the text of key data points. For example, finding where in the text the clinician makes a reference to a diagnosis or prescription. Named Entity Recognition 
(NER) is the task of identifying elements within unstructured text that fall within predefined conceptual categories. This task is at a higher level than simply identifying nouns within a text, because a named entity is understood to have more significance than a nonnamed entity. In chapter 3 , we will discuss this in more depth.

NER is an active area of research in the Natural Language Processing (NLP) community and the efforts of the community have produced many techniques and tools that may prove valuable in addressing the problems with processing unstructured textual data. Many of these techniques have been documented and published in the research literature and open source implementations have been distributed for open use within the community [29][30][31][32].

These NER tools, however, tend to be tuned for performance in a specific application domain. Because of the distinctions in style, language usage and vocabulary across domains (e.g., biomedical journal articles vs. clinical notes vs legal documents), a tool that performs well in one domain may not perform well in another domain. Therefore, in order to effectively use one of these tools in a real world application, one must first identify among the many existing tools a proper one or a combination of multiple tools, and this task is neither trivial nor avoidable.

Additionally, while the community has developed tools that show strong performance on a number of NLP tasks, the impact of these achievements tends to remain within the confines of the research community and it remains a challenge to translate the 
progress into real world use cases and improve the overall capabilities of EMRs in use today.

Among the EMRs in use today is the free, enterprise scale, open source EMR OpenMRS [4]. OpenMRS is implemented on five continents globally and was developed with the goal of improving health care delivery to under-resourced areas of the world.

Our objectives for this project include: 1) Survey the current research in Named Entity Recognition of bio-medical text to identify candidate systems for further consideration. 2) Based on this survey, perform an extensive empirical evaluation of the most promising NER systems and combinations of systems on a test bed of textual clinical notes to identify the system most suited to our project. 3) Use the findings from objective two to develop a module for OpenMRS to structure and present the content of clinical text in such a way as to promote better and faster comprehension by clinicians and, by so doing, free up their time and attention to be better spent on other aspects of patient care.

We undertook this project in two phases. In the first phase we addressed objectives 1 and 2. Based on recent literature, we identified four state of the art NER systems for further evaluation. Those systems include: 1) MetaMap [6] from the US National Library of Medicine. 2) Apache cTAKES [5], developed originally at the Mayo Clinic. 3) BANNER [11], developed at the Arizona State University, and 4) MGrep [12] from the University of Michigan. The evaluations of these systems were performed on hand annotated patient discharge summaries distributed by the I2B 2 group. To perform these 
evaluations, we were required to develop our own evaluation framework. The NER systems are discussed in more depth in Chapter 3.

Informed by the results of these evaluations, we entered the second phase of the project. In this phase, we developed a new module that extends OpenMRS with NER functionality. The Visit Notes Analysis module uses an NER system to extract concepts from textual patient notes in the semantic categories of: Problem, Test, and Treatment. Table 4 in section 4.1 elaborates on these entity classes. The extracted concepts are used to provide snapshot summaries of patient notes that promote fast synthesis of patient history as well as the ability to easily locate information of interest via an intuitive interactive interface.

Additionally, the NER system that we chose as a result of phase 1 allows the developer to train new NER models to perform the NER task. To extend this option to the OpenMRS module, we developed a companion web application, the Visit Note Annotator and Trainer application. This application runs outside of OpenMRS and allows the user to train a new NER model on data from OpenMRS as it becomes available. Because OpenMRS is implemented around the world, regional dialects of the same language may vary drastically and the ability to retrain a model can compensate for these differences. This application provides an interface for the user to review and correct previous annotations. These corrections can then be used to train the new model to improve the performance of the OpenMRS module. 
We encountered several challenges while completing this project. An initial challenge in undertaking evaluations of the NER systems was the heterogeneity of input and output protocols for the set of systems. Although the NLP community has proposed several standards for recording textual annotations that would allow for interoperability of NLP systems, no two systems we evaluated implemented the same standard. This meant that we were required to develop an extensible evaluation framework to accommodate the various protocols. There were also many development challenges associated with integrating the chosen NER system into the OpenMRS platform. These challenges included becoming familiar with the OpenMRS system design and the Java Spring web application framework.

By completing this project, we have made three main contributions. First, we have undertaken an extensive empirical evaluation of four leading Named Entity Recognition systems on a public body of clinical narrative text. The results from these evaluations were fundamental in subsequent design decisions for our module and similar results could not be found in the literature. Therefore, these results will also serve to inform future developers and researchers when they pursue similar projects.

The second contribution is the pair of applications themselves. The Visit Notes Analysis module and the Visit Notes Annotator and Trainer application pair provide a new and valuable way for clinicians to review and navigate the textual Visit Notes recorded about a patient. The incorporation of the Visit Notes Annotator and Trainer application makes our system adaptable and flexible when confronted with regional language 
differences. The pair of applications also serves as initial milestone in the incorporation of NLP research into OpenMRS.

Finally, we contribute an Application Programing Interface that allows developers to easily access the NER functionality in our module. This means that the possible uses of NER in OpenMRS are not limited to just those implemented in our module. Future developers can build on top of our work to make new and better user of the NER capabilities.

The remainder of this document is structured as follows: Chapter 2 describes several Open Source EMRs including OpenMRS as well as several applications of NLP techniques in EMRs and other biomedical domains. Chapter 3 discusses the four different biomedical NER systems under evaluation including their underlying algorithms and target domains as well as various ensemble formations of those systems. Chapter 4 elaborates the evaluation process used to compare the performance of the various NER systems and ensembles under investigation. This includes descriptions of the data sets, evaluation criteria, configurations as well as the evaluation results. Chapter 5, provides an overview of OpenMRS and the two components of our system: the OpenMRS Module and Companion Training Application. Resource usage and system performance metrics will also be discussed. Chapter 6 concludes this thesis by identifying the limitations of our implementation, providing concluding remarks and suggesting future directions for this project. 


\section{Chapter 2}

\section{EMRS: Background and Related Work}

In this section, we provide background on Electronic Medical Record systems (EMRS), their primary functions in medical practice and several EMRS that are currently available. We will also describe several ways in which EMRs have been used to provide higher level support to clinicians as well as discuss applications of NLP within EMRs that have been proposed by the research community. This section will orient the reader to the field in which our work has been undertaken.

\subsection{Open Source EMRs}

An Electronic Medical Record (EMR) system is a patient data repository that supports digital record keeping of patient data within a medical practice. EMRs generally support the point of care needs of a practice such as retrieving, updating, and processing patient records. These tasks can include viewing patient medical history, recording new diagnoses and observations, placing lab orders and ordering prescriptions. Information about these tasks is recorded and maintained for future reference. Thus, an EMR attempts to maintain a complete and accurate record of a patient's interactions with the medical 
facility. As mentioned in the introduction, an EMR tends to enforce a very rigid structure on the patient data that it supports; that is, patient data is entered as encoded concepts to support efficient retrieval. This is often driven by an underlying relational database schema that defines, in advance, every data-field that can be entered. Some EMRs take this prescriptive approach even further and define every data point as a concept and define all relationships and interaction allowed between these concepts. OpenMRS is a proponent of this approach, and we will discuss it further in Chapter 5. These constraints make it difficult to support unstructured data that does not easily fall into the prescribed structures.

As the benefits of transitioning from paper records to EMRs have become clear over the past several decades, more EMR options have been made available on a variety of platforms and at a range of cost. Many enterprise scale EMR systems are available from private vendors such as Epic [44] and Practice Fusion[43]. Benefits from purchasing a proprietary system include technical support and continued development of the system, so long as the vendor remains in business. In proprietary systems, however, the vendor tends to be the sole party responsible for feature development which limits the customization options available to the end users. Also, the cost associated with private vendors often puts them out of reach of smaller or more rural practices, as well as practices in lesser developed countries. Open source EMRs provide an alternative. These EMRs are distributed freely with the source code which allows end users to customize it to their needs, given the proper technical expertise. While many open source projects have an active community of 
developers and implementers, there is no guarantee that the end user has access to technical support or continued development.

There are many free open source EMRs available that provide various services and functionality to practitioners. VistA [19] is an EMR developed by US Department of Veterans Affairs to support its network of VA Hospitals throughout the US. It is one of the oldest and most time-tested systems with wide implementation in the US and internationally which has proven its robustness and reliability. A consequence of its longevity and history, however, is a dated user interface that can lead to usability problems. Furthermore, an architecture implemented in the Massachusetts General Hospital Utility Multi-Programming System (MUMPS) [20] environment can lead to low maintainability and various customization issues which mitigates some of the benefits of open source EMRs.

OpenEMR [21] is another widely adopted open source EMR with over 4,000 downloads per month. The system boasts a US Office of the National Coordinator certification that indicates that it follows interoperability standards. Like VistA, OpenEMR suffers from a dated UI and architecture, but to a lesser degree. It is implemented with PHP/MySQL.

OpenMRS was designed with modularity and customization in mind. It was built as a medical informatics core platform on which to build additional functionality, in the form of modules. An OpenMRS installation generally comes with a core set of modules 
to provide basic features, the installation can then be extended with a number of modules from a growing and community driven module library. OpenMRS is implemented in countries around the world, including Kenya, South Africa, Rwanda, India, China, Haiti, Pakistan and the Philippines [33]. The AMPATH [28] implementation in Kenya serves over 50,000 HIV patients. Figure 1 shows a standard OpenMRS 2.2 Patient Dashboard. 


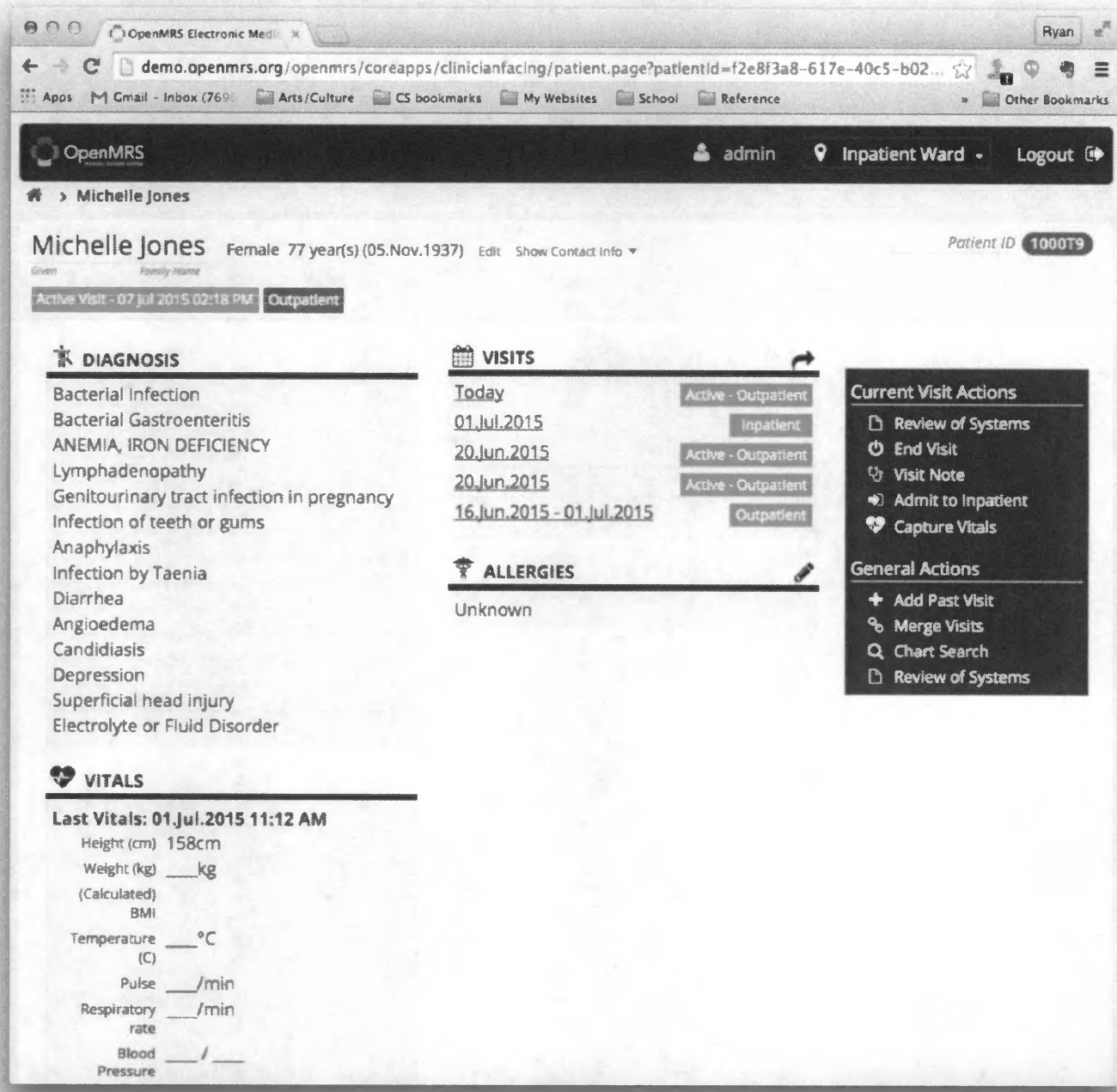

OpenMRS is built on the Java Spring web application framework [34], and is accordingly accessed by the end user through a web browser. At the heart of the system is the OpenMRS Data Model that is accessed through a service layer which leverages the Hibernate Object Relational Mapping system [35]. OpenMRS's use of modern, industry 
standard technologies, design patterns and practices, makes it ideal for the development of our module.

\subsection{Applications of NLP in Point of Care Tools}

The benefits of using an EMR go beyond fast access to patient records. EMRs can provide higher level cognitive support to the practitioners such as triggering notifications or providing reminders after certain events are recorded. Clinical Decision Support (CDS) systems have assisted practitioners for several decades with tools to improve patient care such as automatically suggesting tests after specific therapies have been undertaken [22]. An obvious direction for CDS systems to take would be to apply natural language processing to provide another layer of cognitive support to the practitioners. Although the development of algorithms and systems to perform natural language processing tasks on medical text is an active area of research, means of translating that research into tools for use at the point of care is still an open question. It has been suggested that the closed source nature of proprietary EMRs prevalent today, has contributed to the lack of NLP usage today.

Demner-Fushman et al. recently reviewed many potential applications of NLP in CDS and other EMR functions [24]. These include semi-automatic coding of admit diagnoses and ICD-9 codes [25] as well as automatically extracting interpretations from lung scan reports and monitoring diagnostic performance by radiologists [26]. These 
applications, however, are still being researched and implementations of the research in practice have not been widely reported.

Hirsch et al. described a longitudinal, problem oriented, patient history summarization tool-HARVEST — that operates on top of existing EMR systems [23]. HARVEST aggregates data from clinical notes as well as patient visits (for example, ICD9 billing codes) into the patient summaries, figure 2 shows a screenshot. Its user interface consists of three components: a timeline that provides a graphical view of patient history showing diagnoses and other events, a word cloud depicting problems with font size determined by a computed salience weight, and clinical note navigator. The application provides an interactive visualization of 'problems' identified in the patient history and textual patient notes. Our work resembles HARVEST in many ways. One main difference is that our software system is open source and focuses on structuring clinical notes. We also expand the set of entity classes of interest to include 'tests' and 'treatments.' 
Figure 2: HARIEST Screenshot.

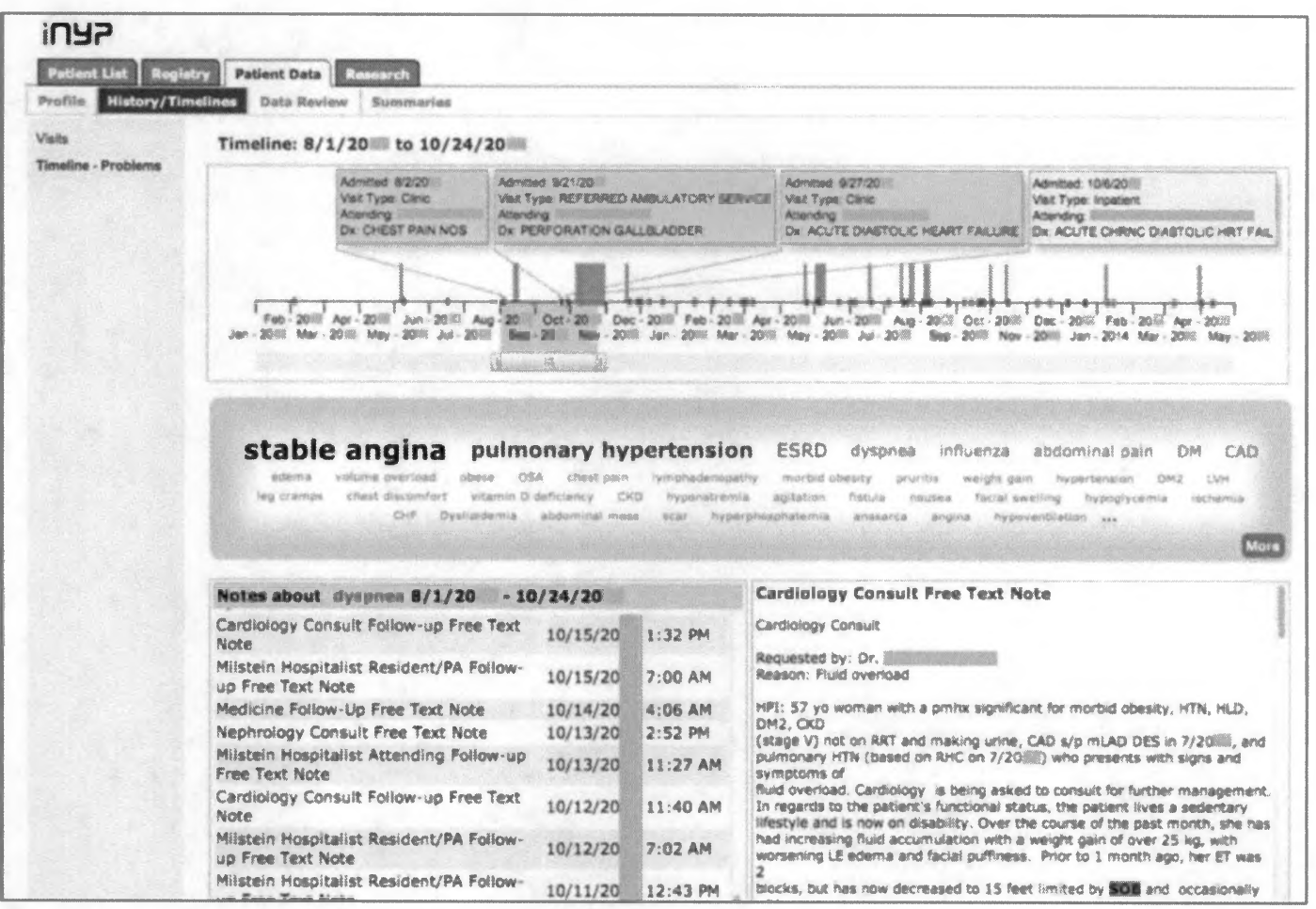




\section{Chapter 3}

\section{Named Entity Recognition Systems}

In order to implement our Visit Notes analysis module, we had a range of NER systems to choose from. We were not required to design our own as NER is a relatively mature field with many open source implementations available. To find the proper system for our module, we conducted a survey of the literature of current NER systems to identify a set of systems for further evaluation. In choosing systems, we considered the reported performance and their target text domain. Table 1 presents an overview of our survey. In this section, we will discuss details about the four NER systems that we evaluated as well as how we constructed ensembles from these systems in an attempt to enhance their performance.

Table 1: Four NER systems: target application domains and highest reported precision/recall.

System Max Precision Max Recall Target Domain

\begin{tabular}{lccl}
\hline cTAKES [5] & .89 & .76 & $\begin{array}{l}\text { Clinical } \\
\text { Narratives }\end{array}$ \\
MetaMap [6] & .85 & .78 & $\begin{array}{l}\text { Biomedical } \\
\text { literature }\end{array}$ \\
BANNER [11] & .85 & .79 & $\begin{array}{l}\text { Biomedical } \\
\text { literature }\end{array}$ \\
MGrep [12] & .88 & .68 & Variable
\end{tabular}




\subsection{Apache Clinical Text Analysis and Knowledge Extraction System (cTAKES)}

The Apache Clinical Text Analysis and Knowledge Extraction System (cTAKES) [5] was developed at the Mayo Clinic in Rochester, Minnesota with the express intent of analyzing clinical narrative text and extracting clinically relevant information. This information includes mappings from text to a lexicon as well as identifying clinical events, relations and negations.

cTAKES implements a six-part analytical pipeline common NER systems. At least three out of the four systems we examine (cTakes, Metamap, and BANNER) implement some variation of this design. Each component of the pipeline contributes metadata in to the text under analysis. In general, subsequent stages in the pipeline perform more sophisticated analysis based on the results of the previous stages. Figure 3 shows a general NER pipeline. While the architecture employed by the NER systems is similar, each stage allows the systems to diverge based on specific implementation decisions. An understanding of this pipeline and system-specific details will provide insight into the performance differences observed during our evaluations (See Chapter 4).

Next, we will describe the general characteristics of this pipeline, and then discuss the specific features employed by cTakes. 
Figure 3: Example NER Analytic Pipeline

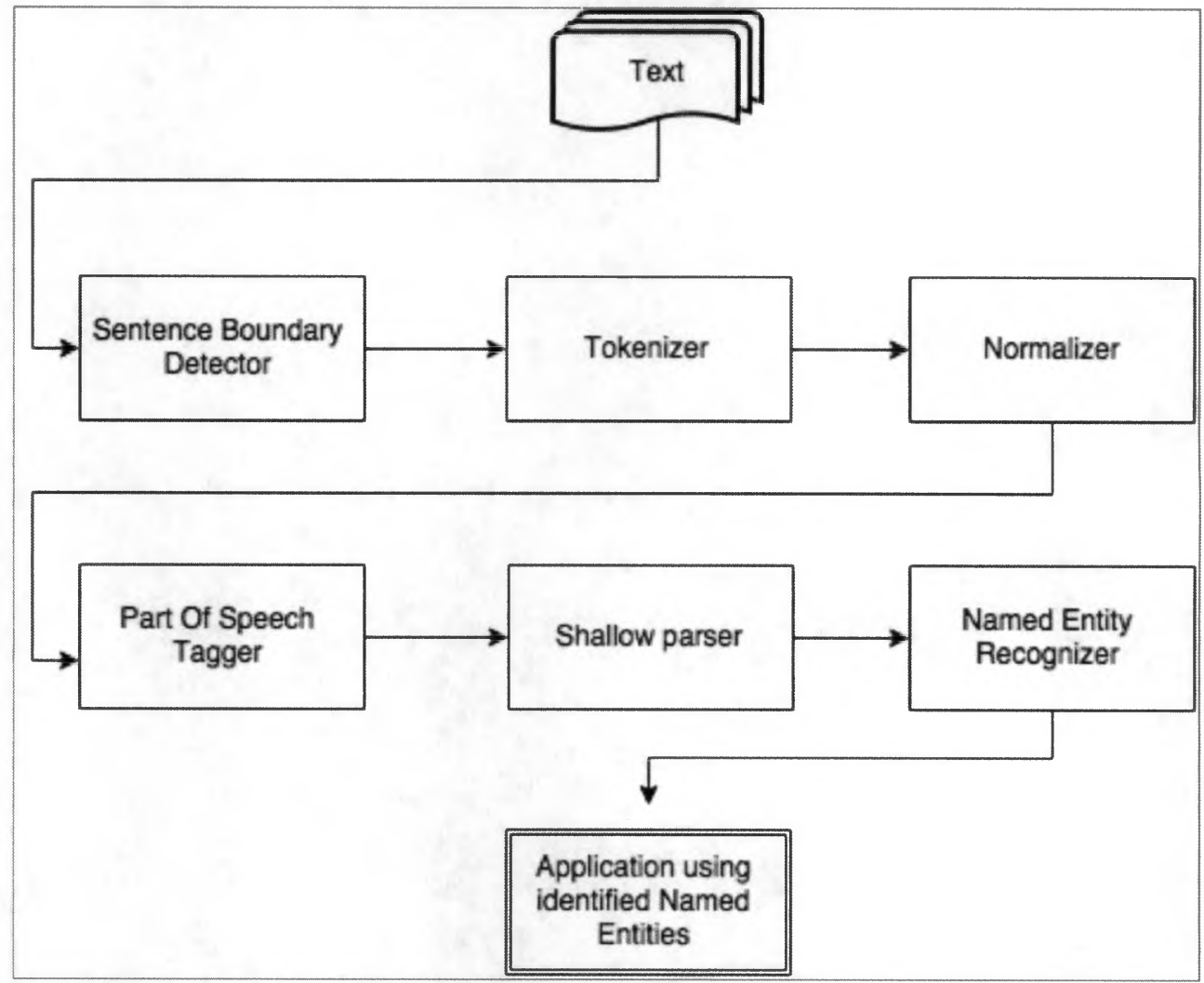

The first stage of the pipeline is the sentence boundary detector which, as the name suggests, segments the input text into sentences by identifying the boundaries. A sentence boundary detector can achieve relatively high accuracy by simply finding common sentence-terminating punctuation marks such as ".,,"!", and "?". This however will not result in segmentation that is $100 \%$ accurate. For example, when an abbreviation such as "Dr." is present in the text, the period will be misidentified as a sentence boundary. Because of this, sentence boundary detectors often employ a machine Learning classifier, such as a 
Maximum Entropy classifier [48] to predict whether or not a punctuation mark indicates the termination of a sentence.

After the Sentence Boundary detection step comes the second step --tokenization-in the pipeline. Tokenization is the process of segmenting a sentence into a stream of individual tokens. A token is generally a word, number, or punctuation mark. For example, the sentence "Dr. Blue prescribed $7.5 \mathrm{mg}$ of penicillin on 5/5/2015." would be properly tokenized into a stream of ten tokens as follows: | Dr. | Blue | prescribed $|7.5| \mathrm{mg} \mid$ of $\mid$ penicillin $\mid$ on $|5 / 5 / 2015| . \mid$. Note that the character '.' is include in the tokens "Dr." and "7.5", however it is its own token at the end of the sentence. A naïve whitespace tokenizer could be developed that separates all tokens based on whitespace and assigns non alphanumeric characters to their own token, for example '.' and ' $/$ '. From the example sentence above, we can see why this naïve tokenizer would not generate the desired output. The string "7.5" would be tokenized into $|7| .|5|$. Similar problems arise with the strings "Dr." and "5/5/2015." Because of this, tokenizers are often augmented with token merging rules that account for common punctuation patterns. A sample rule that would address the date format observed above would state: "merge any string composed of number characters separated by the character ' $/$ ", which recognizes a date string. This rules can often be easily expressed using regular expressions or finite state machines.

The third component of our example pipeline performs the task of token normalization. It is common that two tokens may not contain identical character sequences, yet refer to the same concept. For a concrete example, consider the following three tokens: 
"prescribe," "prescribes", and "prescribed". It is easy to see that each of these tokens are different morphologies of the same term; the verb "to prescribe." The presence of multiple tokens with the same meaning can degenerate the overall performance of the system, therefore normalization attempts to reduce all morphologies or variations of a token into a canonical form. "Stemming" is one normalization technique that attempts to reduce words with a common linguistic root into a single reduced version of the word [45]. This stem does not necessarily need to be the exact linguistic root, so long as words with the common root map to the same stem. Stemming is often performed by executing a number of suffix truncation rules. An adequate stemming result for the three terms above, would be the string "prescribe." Two common stemming algorithms include the Krovetz stemming algorithm [34] and the Porter stemming algorithm [35].

Lemmatization is another normalization technique which attempts to identify linguistic word roots through more refined linguistic analysis [46]. It is often more accurate and more resource intensive than than rule based stemming. For example, a suffix truncation stemmer would not reduce the words "better" and "good" to the same form, however a proper lemmatization would.

Equivalence classing of terms is another token normalization technique. An equivalence class is a set of terms with the same meaning. To perform normalization, any term that is a member of an equivalence class is replaced with a canonical form associated with that class. Equivalence classing can become very important when a token has a large number of synonyms, for example brand names for the same drug. The drug "aspirin" may 
be referred to by any number of the brand names. An equivalence class with a canonical form of "aspirin" might contain the terms: "aspirin", "Caprin", "Zorpin", "Aspergum" and, "Bayer" among many others.

The next step in the pipeline is the Part of Speech (POS) tagger. A POS tagger assigns a syntactic part of speech to each token in the stream. Example parts of speech include noun, proper noun, verb, adverb, adjective, preposition etc. Table 2 shows the part of speech tags for our example sentence.

Table 2: Part of speech tags for example sentence.

\begin{tabular}{|l|l|}
\hline Token & Part Of Speech \\
\hline Dr. & Proper Noun \\
\hline Blue & Proper Noun \\
\hline prescribed & Verb, past participle \\
\hline $\mathbf{7 . 5}$ & Cardinal number \\
\hline mg & Noun \\
\hline of & Preposition \\
\hline penicillin & Noun \\
\hline on & preposition \\
\hline $\mathbf{5 / 5 / 2 0 1 5}$ & Noun \\
\hline
\end{tabular}


Following the POS tagger in the pipeline is the Shallow Parser, also referred to as a Noun Phrase Chunker. The Shallow Parser groups tokens together to form higher level structures, such as phrases. Words are grouped by their function in the sentence, for example sequences of nouns and adjectives might be grouped into noun phrases, verbs and adverbs may be grouped into verb phrases. The sentence "The patient took the capsule of aspirin" contains two noun phrases: "The patient" and "the capsule of aspirin." These noun phrases often serve as targets for further analysis, as we will see.

The last component of the example pipeline identifies named entities within the sentence. Named Entities are defined at a higher level of abstraction than syntactic elements such as noun, verb, and noun phrase. In the sentence "The man walked into Starbucks Coffee," while both "man" and "Starbucks Coffee" are nouns, only the latter would be correctly identified as a Named Entity, potentially of the category "Place." In the sentence "The patient took the capsule of aspirin." One would identify "the capsule of aspirin" as a named entity of medicine, however would not identify "the patient".

Two major approaches to the final step in the pipeline, the actual Named Entity Recognition, are discussed in the literature. The first approach performs Noun Phrase Chunking on the input text and the resulting phrases are used for a dictionary lookup of known Named Entities. This technique requires a-priori knowledge of possible entities that will be encountered. It generally results in higher precision but lower recall. The second approach relaxes this requirement by applying machine learning algorithms such as Conditional Random Fields (CRF). Specifically, CRF is based on a graphical modeling of 
the text [27]. It identifies sequences of tokens that resemble previously identified named entities. With this approach, noun phrase chunking becomes an optional step. In Appendix 5, we provide background on graphical models for NER. CRFs have shown strong performance in the NER task and the evaluations that we perform are consistent with this trend.

The implementation of cTAKES generally follows the pipeline shown in Figure 3. Specifically, cTAKES implements its pipeline as follows: 1) Sentence boundary detector is implemented as an extension of the OpenNLP Maximum Entropy sentence boundary detector, 2) Tokenization is performed by a naïve whitespace and punctuation tokenization step followed by a subsequent merging step that combines tokens into dates, fractions, measurements etc. 3) Normalization is performed by a wrapper around the National Library of Medicine's SPECIALIST lexical tools [36], 4) Part of Speech tagging is performed by the OpenNLP Part of Speech tagger with a specialized model provided by cTAKES, 5) Shallow parsing is also performed by OpenNLP, also with a model provided by the cTAKES developers trained on Mayo Clinic EMR data. 6) Named entity recognition is performed by a dictionary lookup within the resulting noun phrase window. This dictionary is composed of a subset of the Unified Medical Language System (UMLS) that includes SNOMED CT and RxNORM terms. The original target corpus for cTAKES comes from the Mayo Clinic EMR system, which made it the only candidate NER system designed specifically to target Clinical Notes. It is open source, implemented in Java, and supported by the Unstructured Information Management Architecture (UIMA) [28]. 


\subsection{MetaMap, The National Library of Medicine}

The National Library of Medicine developed MetaMap as a tool to provide mappings from terms in biomedical text to concepts that appear in the UMLS MetaThesaurus [47]. Unlike cTAKES, the target domain is not specifically clinical notes and was designed to have general functionality across related biomedical domains, including medical literature.

MetaMap's architecture is similar in principle to cTAKES up until the final stage where MetaMap adds an additional step. For the first components of the pipeline MetaMap makes use of the tools distributed with SPECIALIST lexical tool set. Part of Speech tagging is performed by the Xerox part-of-speech tagger found in SPECIALIST [37]. Normalization is performed by SPECIALIST, as with cTAKES) as well as shallow parsing which is carried out by the SPECIALIST minimal commitment parser. It is at this stage that MetaMap adds an additional step to the pipeline. MetaMap performs Lexical Variant Generation on each term within the noun phrases identified by the shallow parser. This step is an inversion of equivalence class normalization, instead of replacing a term by a canonical form determined by its equivalence class, all members of the class are now associated with the noun phrase. This step broadens the base of possible dictionary matches during the dictionary lookup phase. The dictionary lookup phase uses the UMLS Metathesaurus to identify matches. The output of this process is in the form of possible 
dictionary matches to each noun-phrase ranked by an internal, linguistically inspired ranking scheme.

\subsection{BANNER, Arizona State University}

BANNER departs from the noun-phrase dictionary lookup strategy used by both cTAKES and MetaMap. The foundation of BANNER is the graphical model-based sequence tagging algorithm Conditional Random Fields (CRF). See appendix 5 for more information on CRFs. The stages of the pipeline leading up to the CRF are similar to the initial stages of cTAKES and MetaMap. The BANNER system analyzes text at the sentence level, so Sentence Boundary Detection must be performed in advance by the user, as a preliminary step. Tokenization is performed by a simple whitespace and punctuation tokenizer with no secondary merging step. The Part of speech tagging is provided by a Hepple tagger that comes with the Dragon NLP toolkit [38]. Normalization is performed by the Dragon toolkit's lemmatizer. A number of additional token-level features are also extracted such as suffixes, prefixes, and character n-grams. The use of a CRF does not preclude the inclusion of dictionary lookup based features to provide to the algorithm. However, the developers indicate that the decision to leave this step out is an attempt to promote the generality of the system. The authors observed that including dictionary match features "often have a deleterious effect on recognizing entities not in the dictionary."

Unlike cTAKES and MetaMap, BANNER is not distributed with a trained model to carry out the entity tagging. In BANNER's case, this is a CRF Model, whereas for 
cTAKES and MetaMap, it is a shallow parser model. This design choice is consistent with the developer's preference for a general system that can be tuned to a specific domain. In practice, this means that BANNER must be trained on a corpus of training data that resembles the target data before it can be used for NER tagging. This fact sets BANNER apart from the other systems we've examined so far. cTAKES and MetaMap are both designed to be used off the shelf with little to no tuning by the user and they do not require the preliminary training step.

Another key difference between BANNER and the previously discussed systems, is that BANNER's dictionary-free structure is not designed to provide a mapping from textual mentions to a lexicon. Both MetaMap and cTAKES identify concepts in a text and then establish the link to the outside lexicon, UMLS Metathesaurus in both cases. BANNER does not attempt to provide any such link.

\subsection{MGrep, University of Michigan}

MGrep [12] is a system from the University of Michigan that is noted in the literature for its superior performance compared to MetaMap[39]. MGrep's internal mechanisms are the least transparent of the systems under review here. In correspondence with the authors, they explained that the source code has not been distributed because they have not yet published their research on the project. MGrep is distributed as a binary executable that performs dictionary to text mappings. It relies on a comprehensive lexicon 
to do this mapping. For our evaluations, we provided it with the entire UMLS Metathesaurus.

\subsection{Ensemble Methods}

We combined the four NER systems in different formations to make ensemble NER taggers. This was motivated by the idea that because of the differences in design and philosophy behind the development of the systems, each system would present different strengths and weaknesses. With this in mind, we devised several ensemble methods, five of which are outlined in table 3 .

The ensembles we explored fall into five categories as follows: (1) Equal Voting, where all labels by all members in the ensemble are included in the final result. (2) Two out of Three voting, where a label is included if two of the three members identified it. (3) Three out of Four voting, similar to category 2, but with 4 members and an inclusion threshold of 3 votes. (4) Unanimous Voting, where all members of ensemble must agree on a label. (5) Subordinate Voting, where all the votes from a Master System are included, and the subordinate systems vote on any additional labels, either unanimously, or by majority. Subordinate Voting can be considered a meta-ensemble method because an ensemble method is combined with another system. 
Table 3: This table describes six ensemble formations of the four NER systems under evaluation.

\section{Ensemble Name Description}

BN-MM-ev BANNER and MetaMap equal voting. All labels from each system included in final result.

BN-MM-MG-CT-ev

Equal voting by all four systems. All labels by all systems included in results.

MM-MG-CT-Unan

Only labels unanimously agreed upon by MetaMap, MGrep, and CTakes are uncluded.

BN-sub-1

BANNER subordinate voting. All BANNER labels included. labels unanimously aggreed upon by MetaMap and MGrep also included.

BN-sub-2

All BANNER annotations included. Additional labels included by majority vote between MetaMap, cTAKES, and MGrep

BN-Sub-3

All BANNER Annotations included, only annotations agreed upon unanimously by MetaMap, MGrep, and CTakes also included.

In the following chapter we undertake performance evaluations of the four NER systems and the ensemble configurations described above. The results of these evaluations inform the subsequent development of our Visit Notes Analysis Module. 


\section{Chapter 4}

\section{Named Entity Recognition Systems Evaluations}

In this chapter we describe the evaluation process used to test the performance of the four NER systems and the various ensembles. This includes details about the two evaluation corpora and the metrics that we used to measure the performance. We then present the results of these evaluations. We use these results to choose the proper NER system for use in our Visit Notes Analysis module.

\subsection{Evaluation Corpora}

The four NER systems under evaluation were tested against two biomedical datasets. The first dataset was provided by the Integrating Biology by the Bedside group (I2B2) [13], consisting of 425 de-identified patient discharge summaries that originated as transcriptions of clinician dictations. The summaries were annotated by hand for mentions of entities in the classes of Problem, Treatment and Test. Table 4 provides a short description of these entity classes. Full entity class definitions can be found in [37]. 
Table 4: Description of entity classes

Type

\section{Description}

Problem

Treatment

Test
Phrases that contain observations made by patients or clinicians about the patient's body or mind that are thought to be abnormal or caused by a disease.

Phrases that describe procedures, interventions, and substances given to a patient in an effort to resolve a medical problem

Phrases that describe procedures, panels, and measures that are done to a patient or a body fluid or sample in order to discover, rule out, or find more information about a medical problem.

The second dataset is the Colorado Richly Annotated Full Text Corpus (CRAFT) [14]. CRAFT consists of 67 full text biomedical journal articles from the Pubmed Open Access Subset [40] annotated for mentions of entities from the following seven different biomedical ontologies: Chemical Entities of Biological Interest, Cell Ontology, Entrez Gene, Gene Ontology, NCBI Taxonomy, Protein Ontology, and Sequence Ontology. See Table 5 for more details of these two data sets.

Table 5: Details on the corpora used in the evaluations

\begin{tabular}{lllll}
\hline Corpus & \# Docs & \# Sentences & \# entities & Entity Types \\
\hline I2B2 & 425 & $\sim 43,000$ & $\sim 47,000$ & $\begin{array}{l}\text { Problems, } \\
\text { Treatment, Test }\end{array}$ \\
CRAFT & 67 & $\sim 21,000$ & $\sim 100,000$ & $\begin{array}{l}7 \text { biomedical } \\
\text { ontologies }\end{array}$ \\
\hline
\end{tabular}


A few notable differences between these datasets is the density of concept occurrences. The CRAFT dataset has about half the number of sentences, but double the number of total entities. Also, in CRAFT, the entity sets were not disjoint, meaning that a span of text may have multiple annotations. This was not the case with I2B2. These observations point towards CRAFT being a more complex data set and more difficult to process by NER Systems. Our results later in this chapter reflect this as well.

\subsection{Evaluation Criteria and Metrics}

We tested the output of each NER system against three span-based annotationmatching criteria: Exact Match, Single Boundary, and Any Overlap. Given a gold standard annotation of, "medial 1 cm mass above her knee." An annotation identifying "mass above her knee" as an entity would match under the Single Boundary and Any Overlap criteria, whereas the entity "mass" would only match under the Any Overlap category.

In addition to span-based matching, we also evaluated concept class correctness. If the text "blood pressure" was identified as a "problem" in the gold standard annotation, but the NER system identified it as a "test," then it does not satisfy the concept class correctness criterion.

The standard performance metrics of Precision, Recall, and F1 were recorded based on the above-mentioned matching criteria. Formulas for the measures are provided below: 


$$
\begin{aligned}
\text { Precision } & =\frac{\text { True Positive }}{\text { True Positive }+ \text { False Positive }} \\
\text { Recall } & =\frac{\text { True Positive }}{\text { True Positive }+ \text { False Negative }} \\
F 1 & =2 \frac{\text { Precision } * \text { Recall }}{\text { Precision }+ \text { Recall }}
\end{aligned}
$$

To ensure fair comparison, testing on the I2B2 corpus for all the NER systems was carried out over 395 of the 425 documents, where the remaining 30 documents were used to train the BANNER CRF model. Details on the BANNER learning curve are provided in 4.6. Performance on individual entity classes was also recorded.

Because BANNER is not distributed with a trained model and must be trained per use case, we have included two additional measurements: training time per number of documents, and the learning curve that shows precision, recall, and f1 scores for the test and training set plotted against the size of the training set.

\subsection{Technical Challenges in Evaluating Multiple Systems}

The project required us to evaluate the performance of four different systems against a common body of test data. Given four different systems, we were faced with four different input/output protocols. The NLP community has identified the need for a 
common protocol for encoding textual annotations and the benefits that would come with seamless interchange and comparison of annotations from independent systems are numerous. Many text annotation protocols have been developed and proposed as standards such as the Unstructured Information Management Architecture (UIMA), Resource Description Framework in Attributes (RDFa) [41], and General Architecture for Text Engineering (GATE) [42]. However, there has been no consensus from the community on a common protocol to adopt. Consequently, no two NER systems that we evaluated conformed to the same protocol.

The I2B2 test corpus provides the text and annotations of the text in separate files. The annotations are in a semi-human readable format with one annotation per line of the form: 'c="hyperdense kidneys" $28: 1128: 12|| t=$ "problem." ' This can be read as: The $11^{\text {th }}$ and $12^{\text {th }}$ tokens on line 28 of the document contain the string "hyperdense kidneys" which is identified as a concept of type "problem." cTAKES is built on the UIMA system and therefore, its output conforms to the standard UIMA XML Schema. MetaMap provides two output formats relevant to our project. One format is a string based, human readable output and the other is an XML output in a MetaMap specific schema. There is also another format designed to be consumed by Prolog systems. MGrep has only one output option. it provides all of its Text-Dictionary mappings as a tab separated value (TSV) list. BANNER's annotations are performed programmatically through the API and therefore no default output format was provided and we were required to define our own. We decided to define an output format for BANNER that was consistent with the I2B2 output. 
In order to unify the protocols and move forward with the evaluation and ensemble construction, we developed an extensible, unified evaluation framework in Java. This framework provides a common interface through which to compare heterogeneous annotation results. In order to incorporate a new data format, or new NER system into the framework, the user only needs to provide a Java Class that can read annotations in the required format and that implements an abstract super Class. This way, the evaluator is easily extensible and can be modified to include new formats with minimal effort.

\subsection{Results on the I2B2 corpus}

\subsubsection{Results for Individual Systems}

The following series of tables details the performance measurements of the four systems across the six different categories on the I2B2 dataset. The largest value in each column is shown in bold.

Table 6: Results, No Type Matching, Exact Match

\begin{tabular}{|l|l|l|l|}
\hline & Precision & Recall & F1 \\
\hline cTAKES & .26 & .33 & .29 \\
\hline MetaMap & .42 & .25 & .31 \\
\hline BANNER & $\mathbf{. 7 7}$ & $\mathbf{. 5 9}$ & $\mathbf{. 6 7}$ \\
\hline MGrep & .36 & .26 & .31 \\
\hline
\end{tabular}

Table 7: Results, No Type Matching, Single Boundary

\begin{tabular}{|l|l|l|l|}
\hline & Precision & Recall & F1 \\
\hline cTAKES & .45 & .58 & .50 \\
\hline MetaMap & .76 & .45 & .56 \\
\hline BANNER & .92 & .70 & .79 \\
\hline MGrep & .64 & .47 & .54 \\
\hline \multicolumn{4}{|c|}{44}
\end{tabular}


Table 8: Results, No Type Matching, Any Overlap

\begin{tabular}{|l|l|l|l|}
\hline & Precision & Recall & F1 \\
\hline cTAKES & .59 & .61 & .60 \\
\hline MetaMap & .82 & .48 & .60 \\
\hline BANNER & $\mathbf{. 9 3}$ &. $\mathbf{7 1}$ & $\mathbf{. 8 0}$ \\
\hline MGrep & .80 & .49 & .61 \\
\hline
\end{tabular}

Table 9: Results, Type Matching, Exact Match

\begin{tabular}{|l|l|l|l|}
\hline & Precision & Recall & F1 \\
\hline cTAKES & .22 & .28 & .25 \\
\hline MetaMap & .39 & .23 & .29 \\
\hline BANNER &. $\mathbf{7 1}$ & $\mathbf{. 5 5}$ & $\mathbf{. 6 2}$ \\
\hline MGrep & .32 & .24 & .27 \\
\hline
\end{tabular}

Table 10: Results, Type Matching, Single Boundary

\begin{tabular}{|l|l|l|l|}
\hline & Precision & Recall & F1 \\
\hline cTAKES & .37 & .47 & .41 \\
\hline MetaMap & .70 & .41 & .52 \\
\hline BANNER & $\mathbf{. 8 3}$ & $\mathbf{. 6 4}$ &. $\mathbf{. 7 2}$ \\
\hline MGrep & .55 & .41 & .47 \\
\hline
\end{tabular}

Table 11: Results, Type Matching, Any Overlap

\begin{tabular}{|l|l|l|l|}
\hline & Precision & Recall & F1 \\
\hline cTAKES & .44 & .49 & .46 \\
\hline MetaMap & .75 & .44 & .55 \\
\hline BANNER & $\mathbf{. 8 4}$ & $\mathbf{. 6 4}$ &. $\mathbf{7 5}$ \\
\hline MGrep & .66 & .42 & .52 \\
\hline
\end{tabular}

From the above tables, it is clear that BANNER showed the best performance across all measures and categories. cTAKES consistently had the second best recall and MetaMap consistently had the second best precision. MGrep had middle of the road performance. It often had better recall than MetaMap and better precision than cTAKES. 


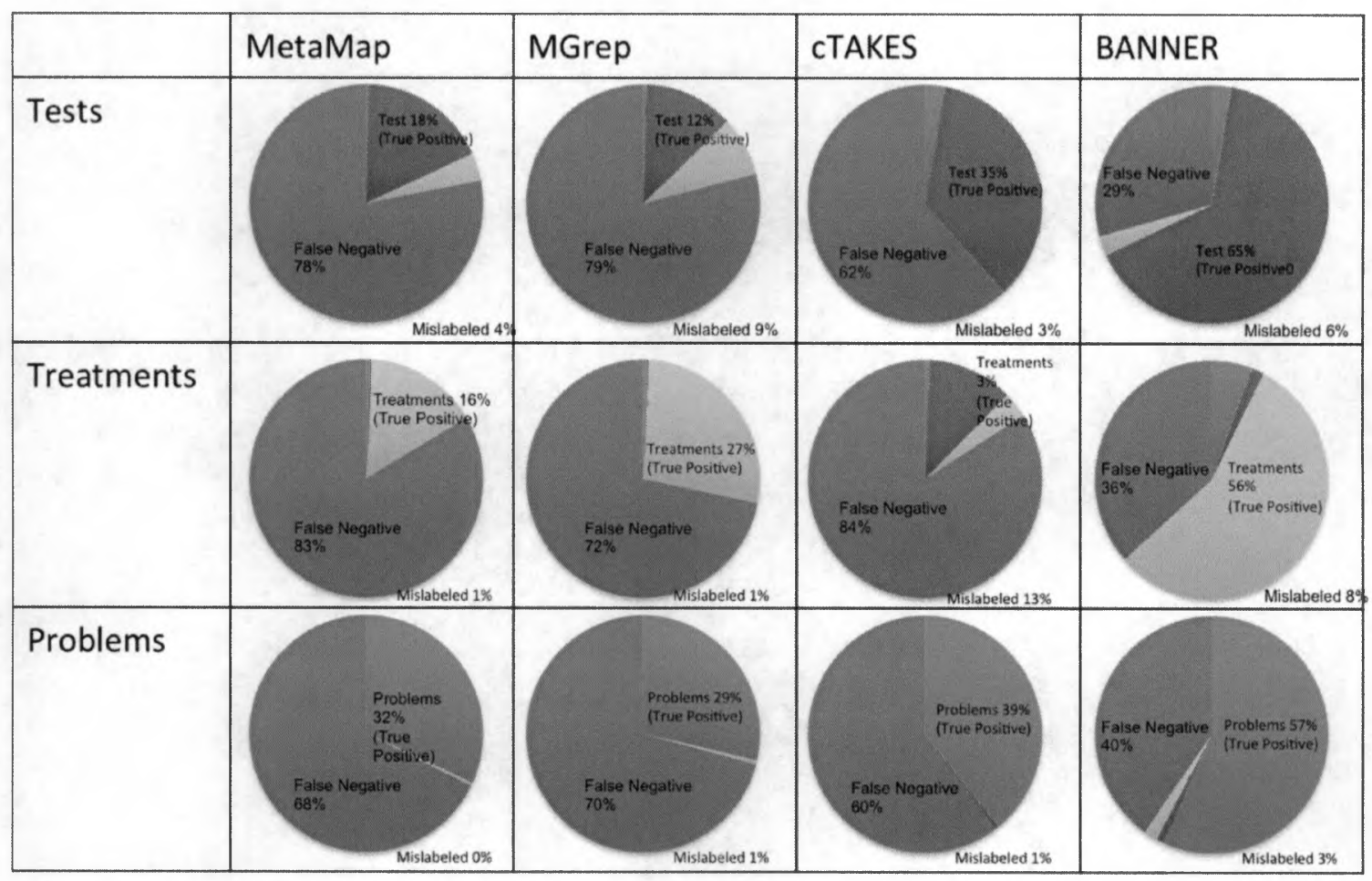

To look further into the performance characteristics of each system, the annotations in the I2B2 dataset were broken down into different groups according to their concept types (i.e., test, problem and treatment). For each of these groups, we evaluated the distribution of annotations provided by the NER systems. The pie chart in the "Tests" row and "MetaMap" column shows the distribution of labels MetaMap identified for the entities labeled "Test" in the gold standard. Here, $18 \%$ of the gold standard annotations were correctly identified, 4\% were incorrectly labeled as "Treatments," and 78\% were not identified at all, which makes them False Negatives. Figure 4 shows the results of this 
analysis. These charts serve as a more granular depiction of the recall performance of each system.

Charts of this kind help us identify strengths and weaknesses of recall for each system. Not surprisingly, BANNER performed the best in each category, as it did have the best overall recall. CTAKES consistently had the second best recall value, which is reflected in the recall breakdown for Problems and Tests, but the chart showing cTAKES's recall of entities in the Treatment category show that it does a poor job labeling them. In fact, it only correctly identifies $3 \%$ and mislabels $13 \%$ of them as tests. MGrep often made the opposite error and showed misclassified Test entities as Treatment entities, showing a $9 \%$ mislabeling rate. MetaMap tended to make a similar mistake as well, however with a $4 \%$ mislabeling rate.

\subsubsection{Results for Ensemble Systems}

The large number of combinations of voting schemes and NER systems preclude us from providing all of the results, so we present some of the highlights of these evaluations. Tables 10 through 15 show the results of the ensemble evaluations. Table 3, (printed here again from Chapter 3) details the different ensembles. 
Table 3 [reprinted from Chapter 3] This table describes six ensemble formations of the four NER systems under evaluation.

\begin{tabular}{ll}
\hline Ensemble Name & Description \\
\hline BN-MM-ev & $\begin{array}{l}\text { BANNER and MetaMap equal voting. All labels } \\
\text { from each system included in final result. }\end{array}$ \\
\hline BN-MM-MG-CT-ev & $\begin{array}{l}\text { Equal voting by all four systems. All labels by all } \\
\text { systems included in results. }\end{array}$ \\
MM-MG-CT-Unan & $\begin{array}{l}\text { Only labels unanimously agreed upon by MetaMap, } \\
\text { MGrep, and CTakes are uncluded. }\end{array}$ \\
\hline BN-sub-1 & $\begin{array}{l}\text { BANNER subordinate voting. All BANNER labels } \\
\text { included. labels unanimously aggreed upon by } \\
\text { MetaMap and MGrep also included. }\end{array}$ \\
\hline
\end{tabular}

BN-sub-2

All BANNER annotations included. Additional labels included by majority vote between MetaMap, cTAKES, and MGrep

BN-Sub-3

All BANNER Annotations included, only annotations agreed upon unanimously by MetaMap, MGrep, and CTakes also included. 
Table 12: No Type Matching, Exact Match

\begin{tabular}{|l|l|l|l|}
\hline Ensemble Type & Precision & Recall & F1 \\
\hline BN-MM-ev & .52 & .71 & .60 \\
\hline BN_MM_MG_CT & .29 &. $\mathbf{7 6}$ & .41 \\
\hline MM_MG_CT_Unan & .57 & .14 & .22 \\
\hline BN-Sub-1 & .55 & .68 & .61 \\
\hline BN-Sub-2 & .41 & .71 & .51 \\
\hline BN-Sub-3 & $\mathbf{. 6 9}$ & .67 & $\mathbf{. 6 8}$ \\
\hline
\end{tabular}

Table 13: No Type Matching, Single Boundary

\begin{tabular}{|l|l|l|l|}
\hline Ensemble Type & Precision & Recall & F1 \\
\hline BN-MM-ev & .61 & .85 & .71 \\
\hline BN_MM_MG_CT & .34 & $\mathbf{. 9 0}$ & .49 \\
\hline MM_MG_CT_Unan & $\mathbf{. 8 7}$ & .29 & .44 \\
\hline BN-Sub-1 & .59 & .82 & .69 \\
\hline BN-Sub-2 & .46 & .85 & .59 \\
\hline BN-Sub-3 & .77 & .81 &. $\mathbf{7 9}$ \\
\hline
\end{tabular}

Table 14: No Type Matching, Any Overlap

\begin{tabular}{|l|l|l|l|}
\hline Ensemble Type & Precision & Recall & F1 \\
\hline BN-MM-ev & .83 & .85 & .84 \\
\hline BN_MM_MG_CT & .58 & .91 & .71 \\
\hline MM_MG_CT_Unan & .93 & .31 & .46 \\
\hline BN-Sub-1 & .88 & .83 & .85 \\
\hline BN-Sub-2 & .80 & .86 & .83 \\
\hline BN-Sub-3 & .91 & .82 & .86 \\
\hline
\end{tabular}


Table 15: Type Matching, Exact Match

\begin{tabular}{|l|l|l|l|}
\hline Ensemble Type & Precision & Recall & F1 \\
\hline BN-MM-ev & .48 & .66 & .56 \\
\hline BN_MM_MG_CT & .27 & .71 & .39 \\
\hline MM_MG_CT_Unan & .58 & .11 & .19 \\
\hline BN-Sub-1 & .52 & .63 & .57 \\
\hline BN-Sub-2 & .43 & .66 & .52 \\
\hline BN-Sub-3 & .66 & .62 & .64 \\
\hline
\end{tabular}

Table 16: Type Matching, Single Boundary

\begin{tabular}{|l|l|l|l|}
\hline Ensemble Type & Precision & Recall & F1 \\
\hline BN-MM-ev & .57 & .79 & .67 \\
\hline BN_MM_MG_CT & .32 & $\mathbf{. 8 5}$ & .46 \\
\hline MM_MG_CT_Unan & $\mathbf{. 8 9}$ & .23 & .36 \\
\hline BN-Sub-1 & .56 & .76 & .64 \\
\hline BN-Sub-2 & .47 & .78 & .59 \\
\hline BN-Sub-3 & .74 & .73 & .74 \\
\hline
\end{tabular}

Table 17: Type Matching, Any Overlap

\begin{tabular}{|l|l|l|l|}
\hline Ensemble Type & Precision & Recall & F1 \\
\hline BN-MM-ev & .74 & .80 & .77 \\
\hline BN_MM_MG_CT & .45 & $\mathbf{. 8 6}$ & .59 \\
\hline MM_MG_CT_Unan & $\mathbf{. 9 3}$ & .24 & .38 \\
\hline BN-Sub-1 & .77 & .76 & .77 \\
\hline BN-Sub-2 & .72 & .79 & .76 \\
\hline BN-Sub-3 & .83 & .74 &. $\mathbf{7 8}$ \\
\hline
\end{tabular}


From the above results, we can make the following observations. Intuitively, the ensemble that required MetaMap, MGrep, and cTAKES to agree unanimously showed the highest precision in four of the six categories. This is likely because it has one of the most restrictive inclusion thresholds. Similarly, the ensemble with the least restrictive inclusion threshold, that which includes all labels from all systems, shows the highest recall values across the board.

In all the categories, the highest F1 score was achieved by the ensemble that included the unanimous voting results of MetaMap, MGrep, and cTAKES along with all labels provided by BANNER. This ensemble effectively combines the system with the best overall performance, with the system that has the best precision. When comparing the ensemble performance against that of BANNER alone, we find that BANNER alone scores a higher F1 measure in 4 of 6 categories, the two categories it does not perform best are the two with the least restrictive boundary matching criterion.

\subsection{Results on the CRAFT Corpus}

We evaulated MetaMap, MGrep and BANNER on the CRAFT dataset using the same matching criteria as with the I2B2 set. We did not evaluate cTAKES because its output entity class labels of Problem, Treatment, and Test could not be easily or adequately mapped to the CRAFT entity classes. Because the BANNER model needs to be trained per use case, we trained a model using the CRAFT corpus, however the demanding training process of BANNER coupled with the density and variety of annotations in the CRAFT 
set limited the number of documents we could use for training. We reached the limits of our resources at training on 9 CRAFT documents. We evaluated BANNER with this 9document model, as well as the model previously trained on the I2B2 corpus. Figure 5 shows the results of these evaluations.

Figure 5: Results on CRAFT Data Set

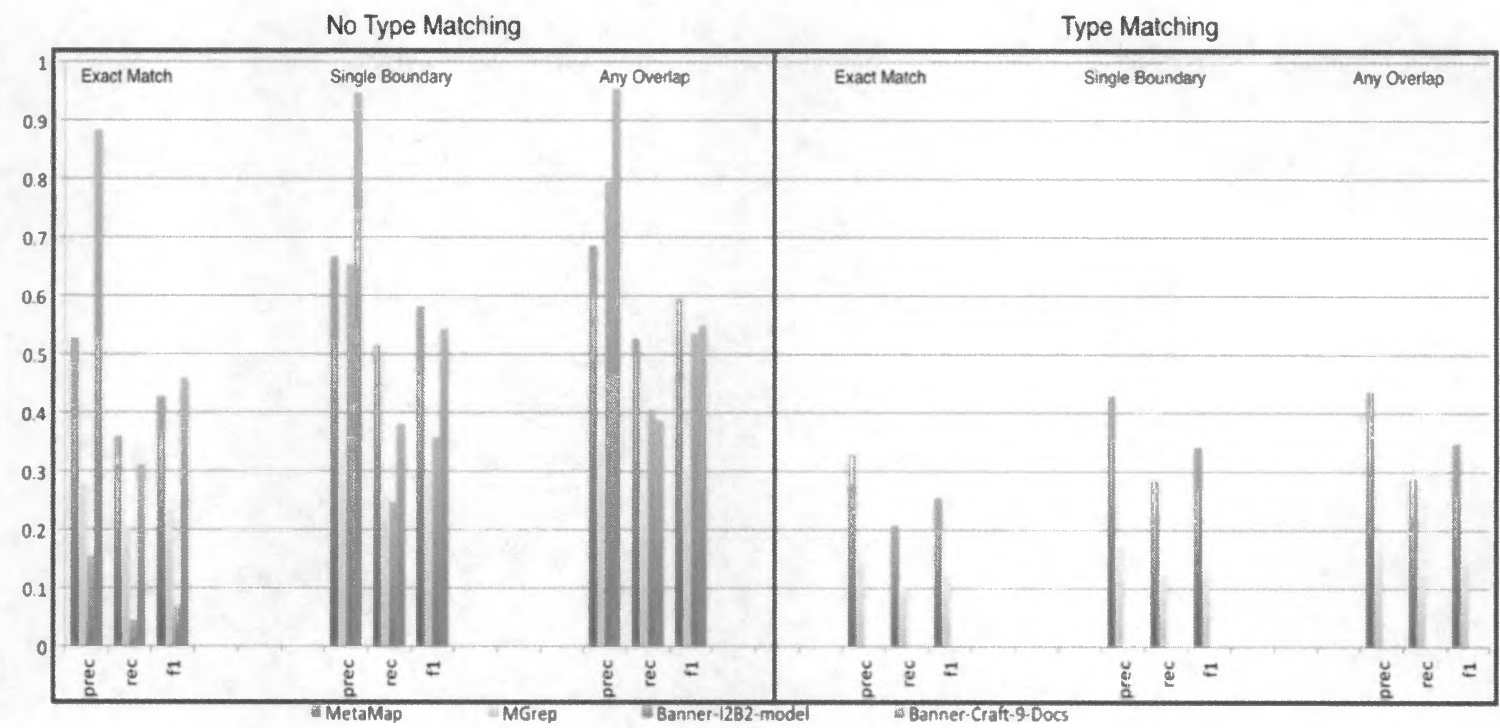

The results show that the MetaMap has the best overall F1 score in all categories except for Exact Match with No Type Matching. and consistently strong performance relative to the other systems in with regard to precision and recall. BANNER trained on nine CRAFT documents shows the best precision when type matching is not required. When type matching is a requirement, both BANNER models show very poor performance. So poor, in fact, that the bars are not visible in the chart. 


\subsection{BANNER Learning Curve and Training Time}

We plotted the learning curve of BANNER's performance on I2B2 data to observe the effect of training set size on performance. Figure 6 shows these results. As expected, BANNER's performance on the test set improves as the training set increases and seems to be converging with the training set at some asymptote. This figure gives us confidence that we would be able to improve BANNER's performance on the CRAFT dataset if we had the resources to train it on a larger set. Figure 7 plots the time it takes to train a new BANNER model on I2B2 data.

Figure 6: BANNER learning curve, shows the precision, recall and Fl scores on the training set and the test set as the size of the training set is increased.

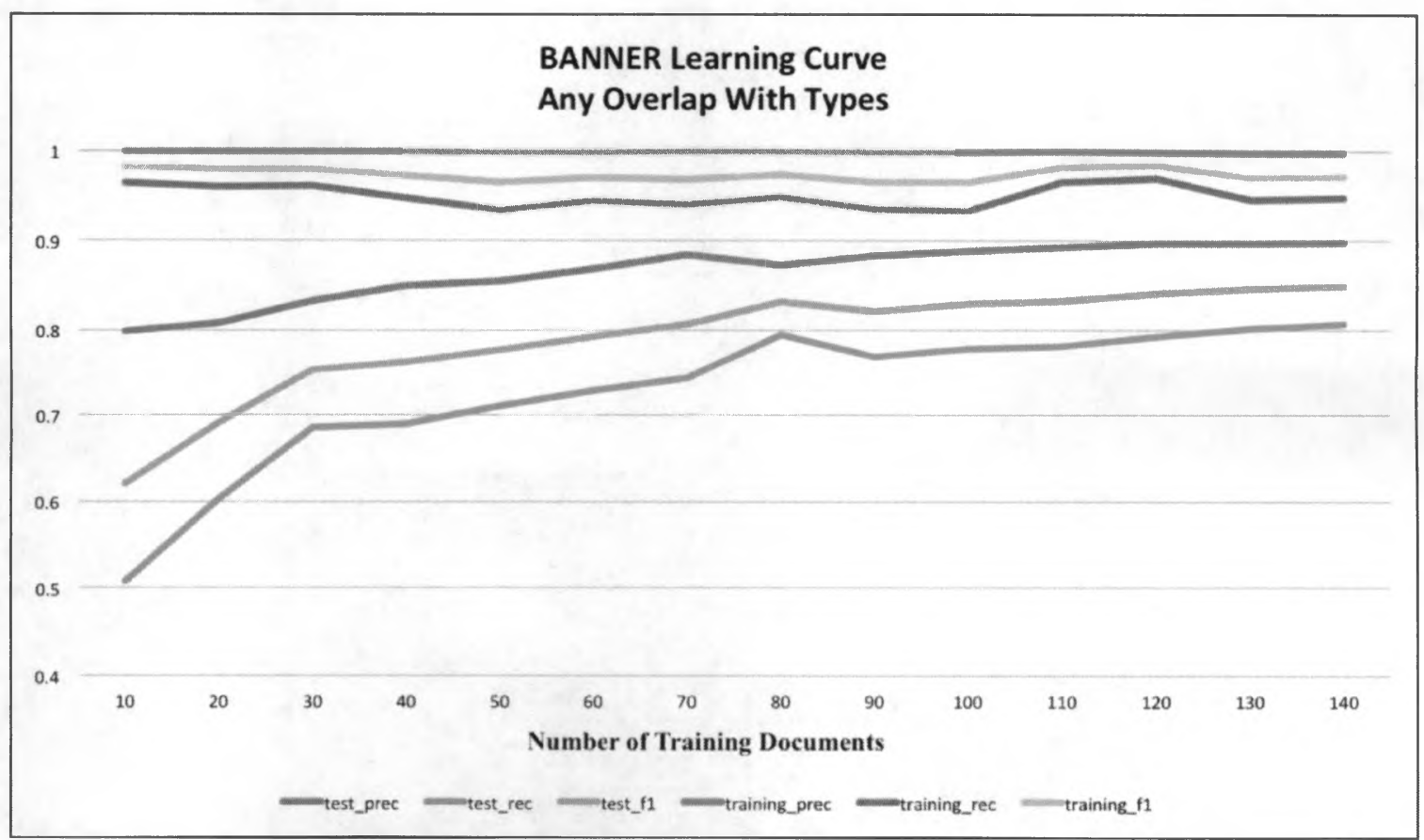




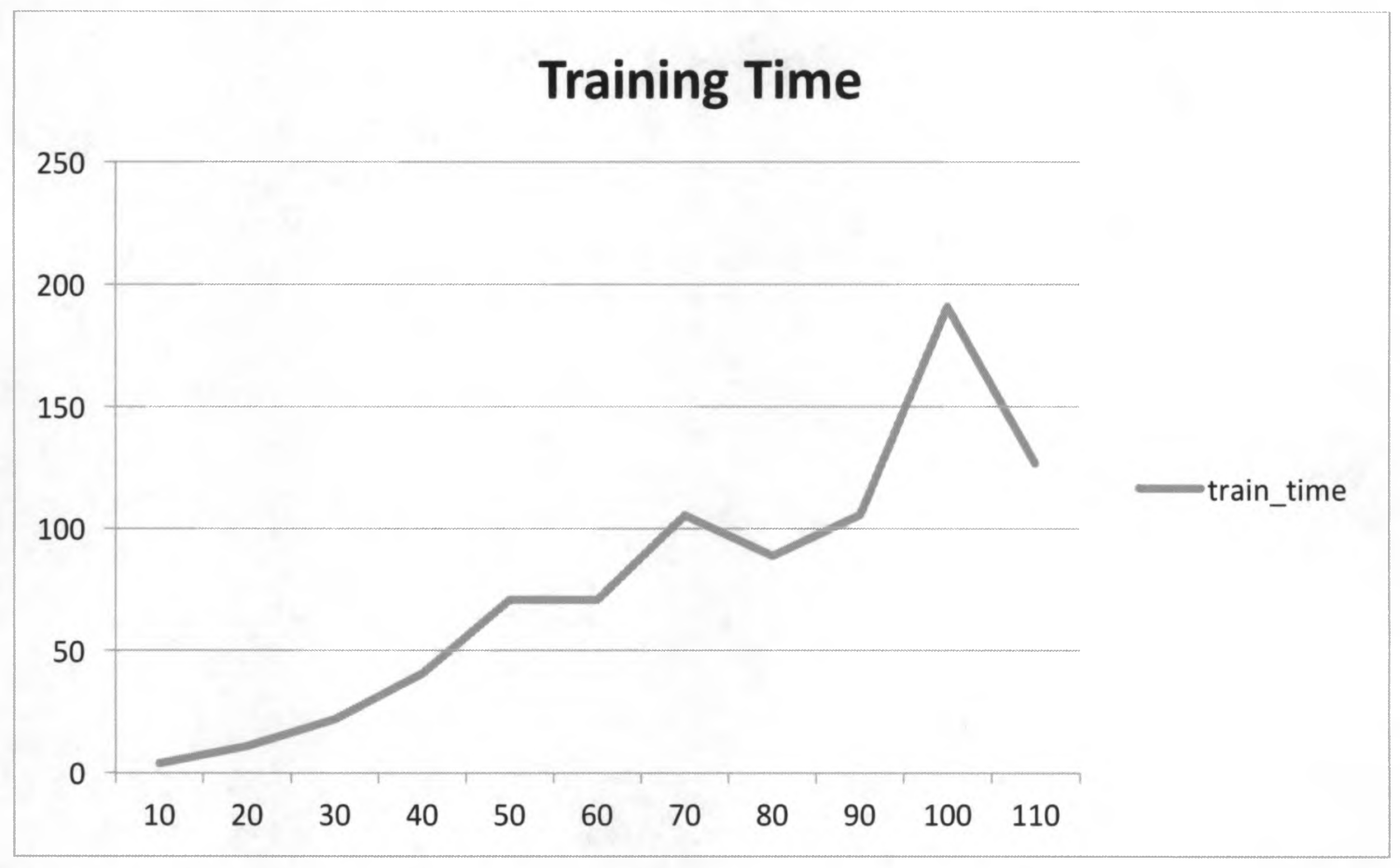

\subsection{Conclusions}

The developers of BANNER indicate that many of their design choices were guided by a preference for generality over specificity, which resulted in the exclusion of dictionary-based features. Also, the fact that BANNER is not distributed with a trained model which forces the user to train his or her own means that it can, in principle, be more easily adapted and tuned to a specific domain than cTAKES or MetaMap. Because cTAKES and MetaMap are open source, it is not out of the question to provide new models to these systems trained on a new target corpus, and provide a new dictionary with terms that are likely to be found in the target. However, these tasks are quite laborious and 
expensive. Thus we have chosen to proceed with BANNER as our NER system of choice in the implementation stages of this project.

One problem with BANNER's training requirement is the necessity of an adequate training set. It is this observation that motivated our development of a web based model training application to allow the end user to iteratively train new BANNER models as new data is generated. This application is described in Chapter 5 as well as the Implementer's Guide appendix entry.

In the next chapter we describe the implementation of the Visit Notes Analysis OpenMRS module built on top of BANNER and the companion Visit Notes Annotator and Trainer application that allows the user to train a new BANNER model. We will discuss major design decisions and architectural components of the applications. 


\section{Chapter 5}

\section{Implementation of Clinical Notes Module}

In this chapter, we describe the major implementation strategies utilized to realize the clinical notes-processing module in OpenMRS. This module consists of two main components: (1) an NER pipeline for the processing of clinical notes with an associated data model, as well as an intuitive user interface to visualize and navigate the results of the NER pipeline; and (2) a web application used for retraining the underlying BANNER model using new and hand-corrected texts. These two components work together to provide optimal performance for the OpenMRS users.

\subsection{OpenMRS Visit Notes Analysis Module}

The OpenMRS core system is designed to support a modular architecture. The core system provides a service layer that serves as the gateway to the underlying data model. All functionality is composed of modules that are built on top of and extend this service layer.

The Concept Dictionary is the fundamental building block of every OpenMRS implementation [15]. It contains names, codes and attributes for every data point or 
observation made in the system. Concepts include medical tests, drugs, results, symptoms, etc. The base OpenMRS implementation generally ships with a default Concept Dictionary that provides mappings to international standards such as ICD-10 [16], SNOMED CT [17], and RxNORM [18]. The dictionary can be customized for each OpenMRS implementation. Concepts can be further grouped into concept classes, which we leverage in our module. An exemplar concept class is 'Diagnosis' which contains concepts such as 'asthma,' 'gallstones,' and 'rickets.' Another example is the class 'Test' which contains concepts such as 'Arm X-Ray' and 'Ultrasound.'

By following OpenMRS's modular design philosophy, we provide an NER API to perform the text analysis in addition to providing an extension to the service layer API that allows for persistence and retrieval of the results.

\subsubsection{Training the Default BANNER Model}

The Visit Notes Analysis module ships with a default model used by BANNER to perform the NER tagging. To train the model, we used 130 annotated documents from the I2B 2 corpus. Annotated sentences from all documents are essentially concatenated into a large documenting for the algorithm to learn from. Figure 8 shows this process at a high level. 


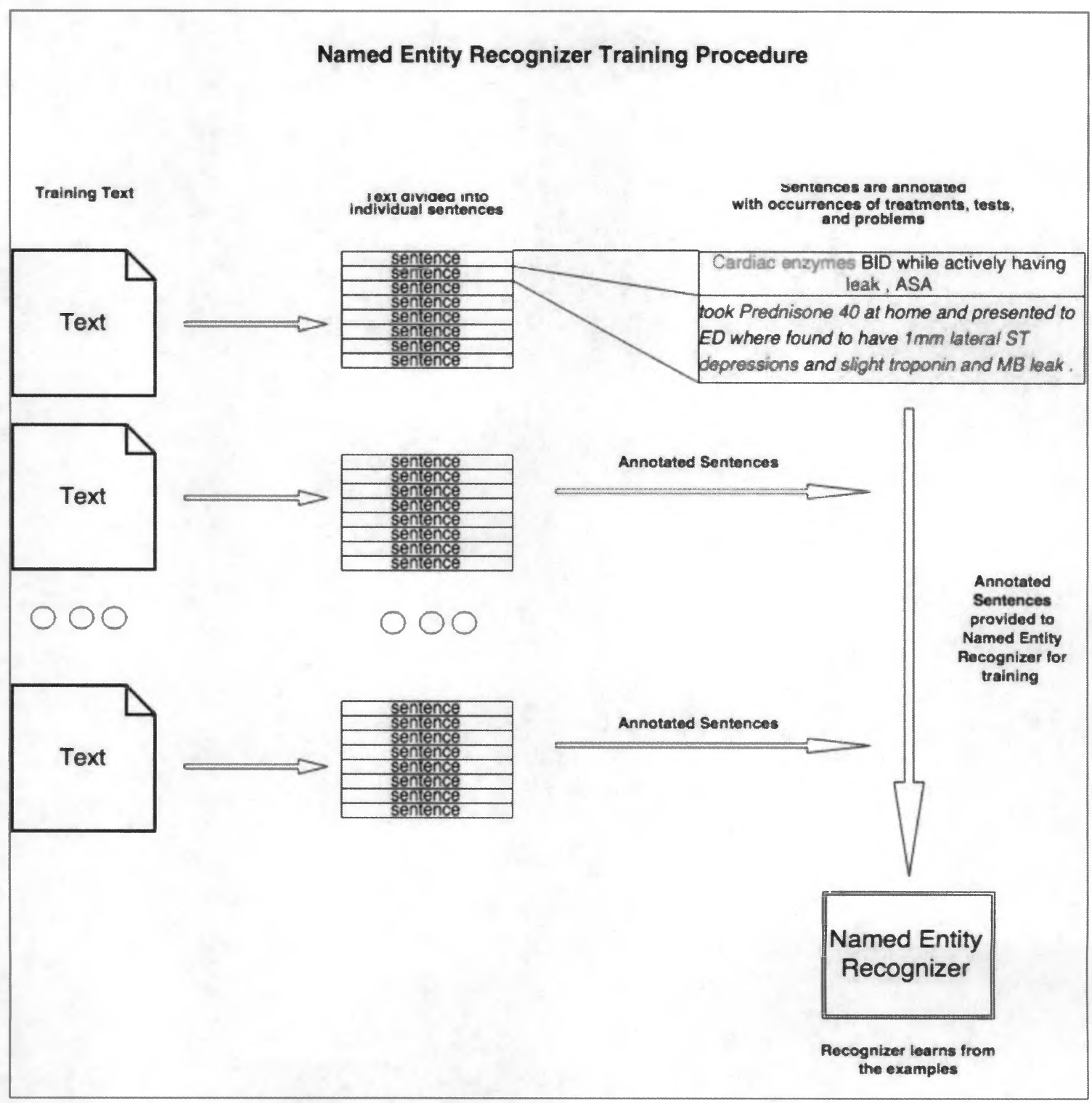

The BANNER library provides an API to carry out the training. Code Listing 1 shows the procedure for initializing a BANNER Sentence object with some text and then annotating it with one annotation. 
Code Listing 1: BANNER API for training a new model

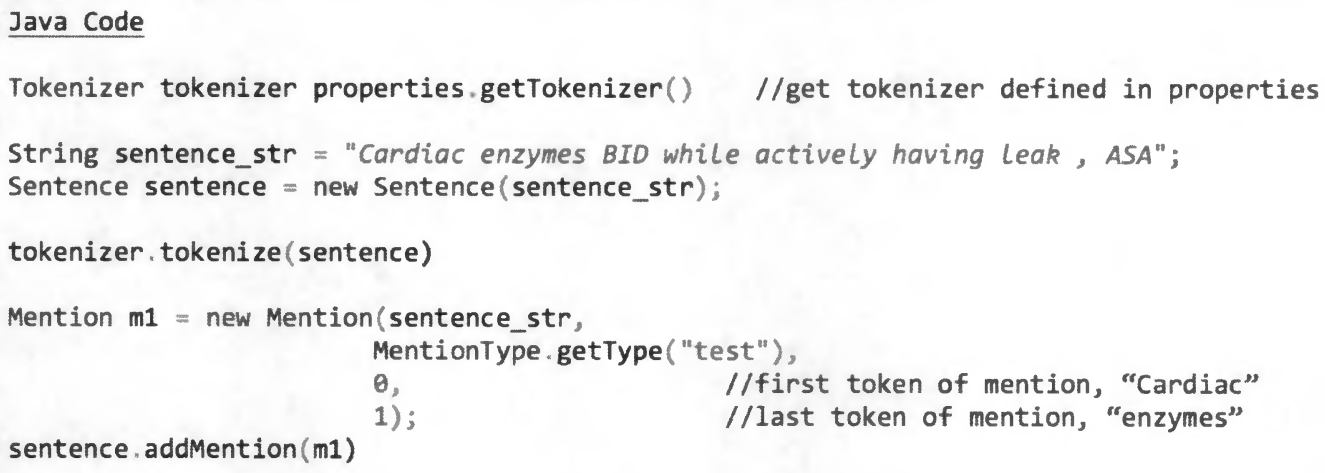

Code Listing 2: BANNER API continued, carrying out training with CRFTagger.train() emthod

CRFTagger tagger $=$ CRFTagger train(sentences, $\ldots$ [properties specified in BANNER docs])

When all documents have been rendered as Sentence objects, they are passed to a training method that uses the training data to compute a new model. Code Listing 2 shows this. The return value is a CRFTagger objects that can perform the CRF tagging of a string of text. It is this object that we use in the Visit Notes Analysis module as part of the NER Algorithm described in section 5.1.3 We also provide a Visit Notes Annotator and Trainer application for training a new model on Visit Notes data from OpenMRS, this process is described in section 5.2.2. 


\subsubsection{Module Data Model}

This module adds four database tables to OpenMRS. The data stored in these tables is accessible via the service layer through the service class NLPService. The User Interface of the module is built on top of the NLPService. Figure 9 shows the extensions our module makes to the OpenMRS data model. A description of the data model is to follow.

Figure 9: Visit Notes Analysis Module Data Model accessible through the NLPService class

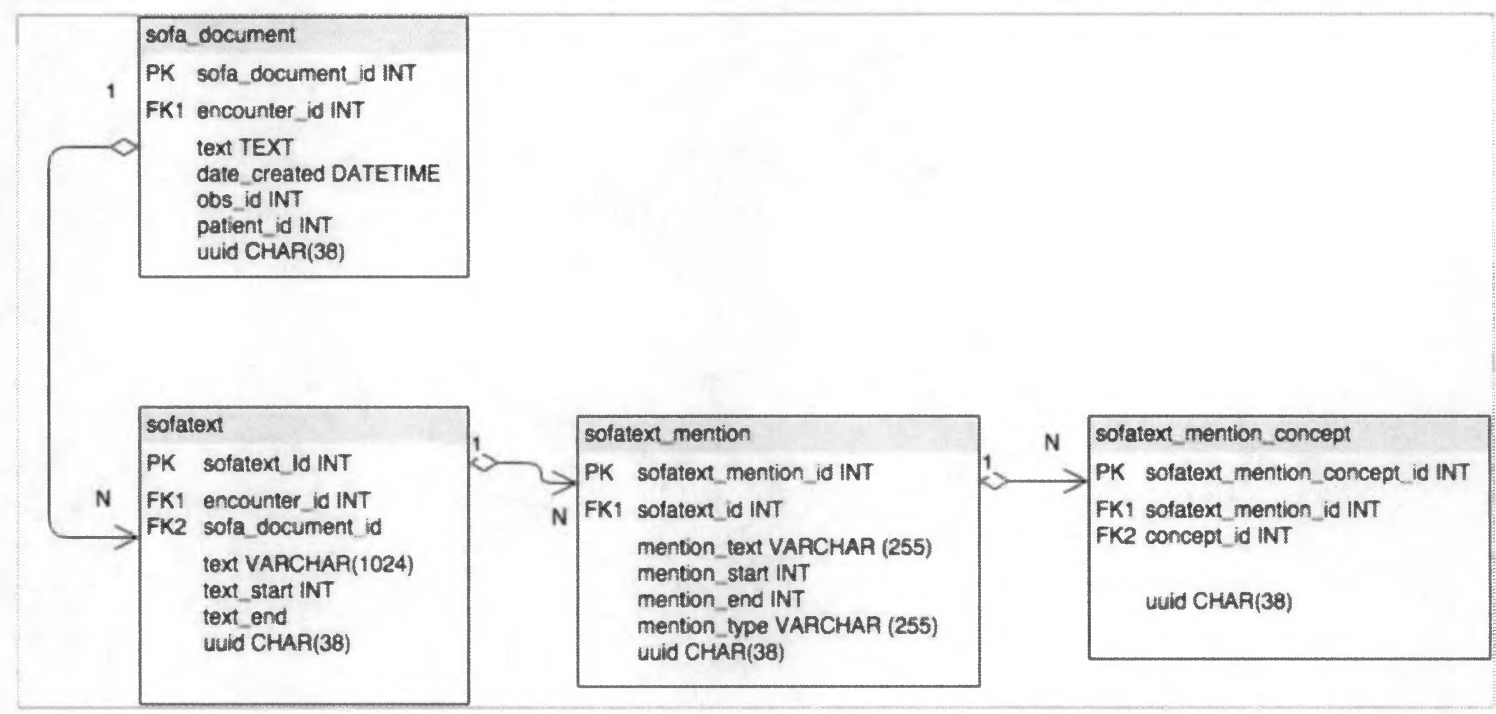

An entry in the sofa document ('sofa' is shorthand for Subject of Analysis) table is the root of a hierarchical data structure that contains all the information generated throughout the process of analyzing a visit note. One sofa_document represents one visit note. During the analysis, the sofa_document is segmented into sentences with a punctuation based sentence segmentation algorithm. These sentences are represented by entries in the sofatext table and contain the text of the sentence as well as indexes that 
indicate where the sentence is located in the original document. The Named Entity Recognition is performed at the sentence, or sofatext, level, which results in a list of entities, or mentions. These results are stored in the sofatext_mention table that maintains information about the location of the mention in the sofatext as well as the mention type and the text of the mention. Finally, if the mention was identified as a result of the first Concept Dictionary matching step (see section 5.1.2), then there is an OpenMRS concept that corresponds to the mention, this relationship is stored in the sofatext_mention_concept table.

The Hibernate database interface provides Object-Relational Mappings between the persistent data and objects in use at runtime in an OpenMRS deployment. Accordingly, the SofaDocument Java objects at runtime reflect the same hierarchical structure of the database model. Figure 10 illustrates the relationships between the four primary data classes: SofaDocument, SofaText, SofaTextMention, and SofaTextMentionConcept. Figure 11 shows a concrete example of how a document and its annotations are represented using the Object Relational Mapped objects. Here, the SofaDocument is composed of two sentences, so the text is segmented into two SofaText objects. These SofaText objects each contain two SofaTextMention objects because the NER Algorithm identified two entities in each. Only "Hepatitis C cirrhosis" was identified using the OpenMRS Concept dictionary. Therefore, only the SofaTextMention object representing it has a reference to a SofaTextMentionConcept. 
Figure 10: Data Model Object Hierarchy

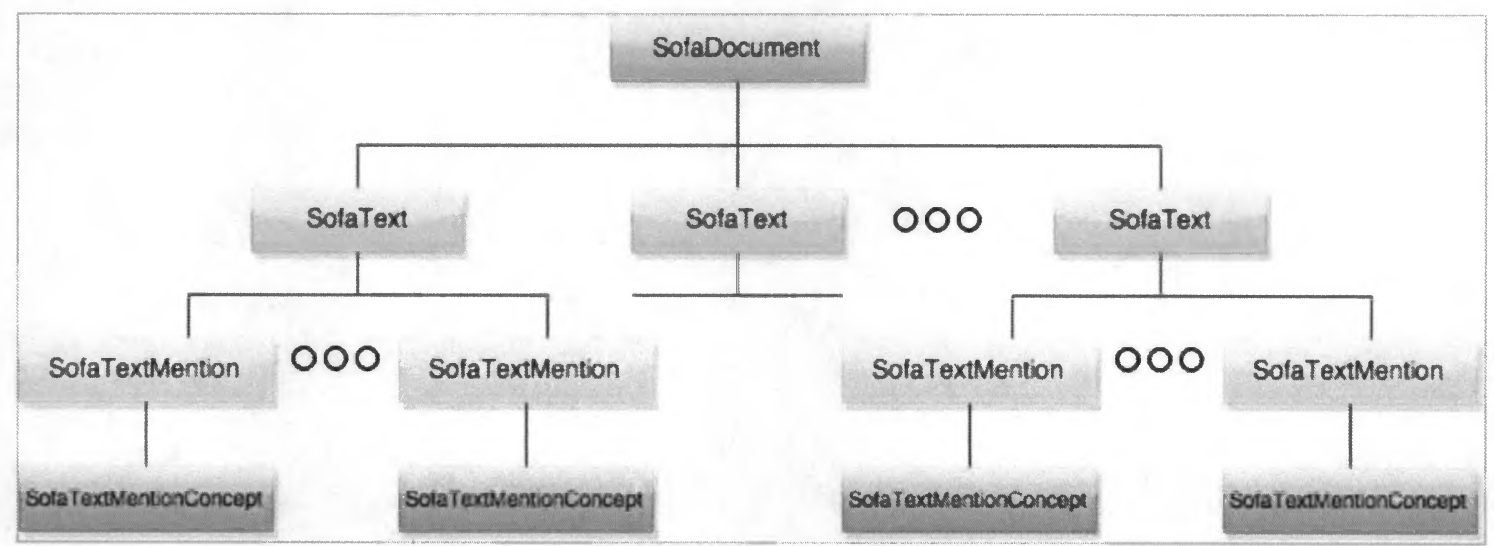

Figure 11: Example of document representation

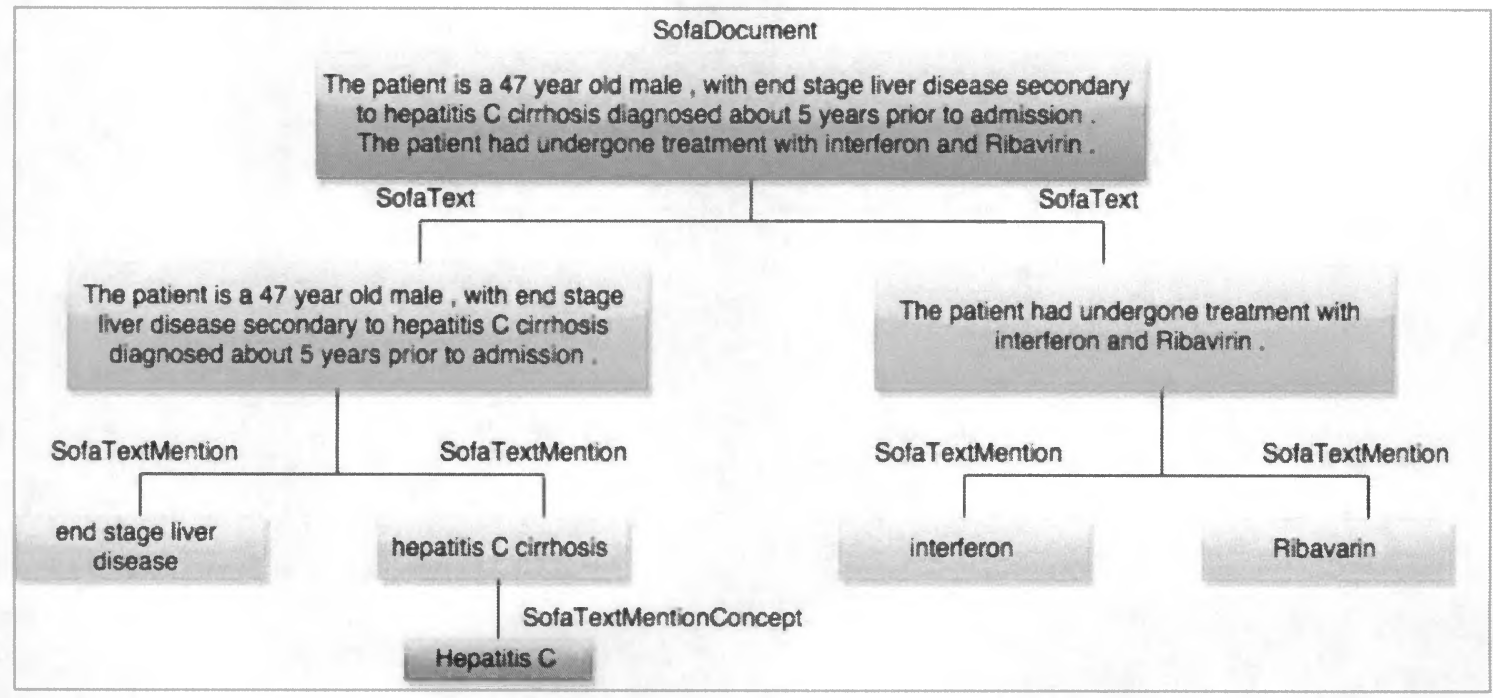




\subsubsection{NER Algorithm and Implementation}

The NER algorithm that is exposed through the API is composed of a two-step analysis sequence that ties together implementation-specific concept classifications and the BANNER analysis. Specifically, the first step performs string-based matching using OpenMRS's concept dictionary and the second step uses the BANNER CRF algorithm.

For the first step there must be a mapping between OpenMRS Concept Classes and the entity types our system identifies. Because our module tags entities into three distinct entity types but an OpenMRS implementation often has many more concept classes, we allow the user to provide a many-to-one mapping from OpenMRS concept classes to entity type. See Table 18 for the default concept class mapping adopted in our module. Given a mapping, our module will use simple string-based matching to find any mentions of OpenMRS concepts and map them to the corresponding class used in our module (e.g., "Problem.").

Table 18: OpenMRS Concept Class to Entity Type Mappings

\begin{tabular}{ll}
\hline Entity Type & OpenMRS Concept Classes \\
\hline Problem & Diagnosis \\
& Symptom \\
& Symptom/finding \\
Treatment & Drug \\
Test & Procedure \\
\hline
\end{tabular}


Using Figure 9 as an example, the concept "Hepatitis C" exists in the OpenMRS Concept Dictionary and has been placed in the class "Diagnosis." See figure 10. Per the Entity Type mapping above, when "Hepatitis C" is identified in the text, it will be mapped to the entity type Problem.

While performing this matching step, the algorithm looks at all Concepts in the different classes as well as all synonyms associated with those concepts. This is important because a concept may have many synonyms, for example the concept "Aspirin" may have many synonyms such as "Bayer," "Aspergum", and "Caprin" that are listed in the Concept Dictionary. Moreover, synonym lists may include words in many languages that refer to a Concept. This means that, if an OpenMRS deployment has Swahili Synonyms for many concepts in the dictionary, our algorithm will be able to find these Swahili terms.

Figure 12: Hepatitis C Entry in Concept Dictionary

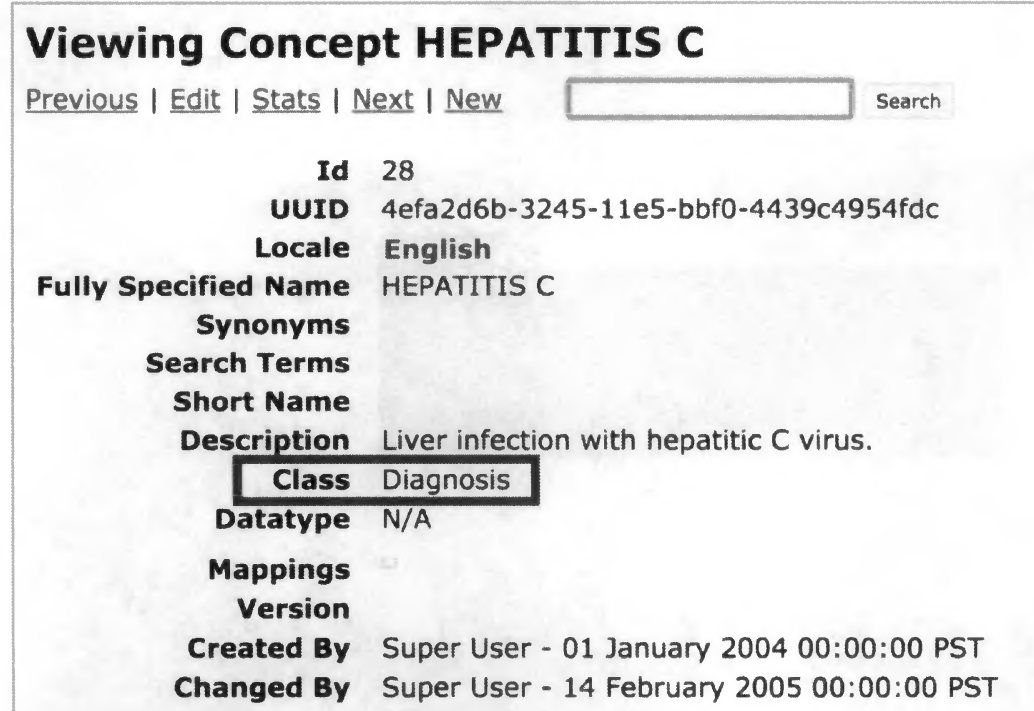


Following the Concept Dictionary based matching, we execute the dictionary agnostic NER algorithm in BANNER. The BANNER CRF allows us to identify entities that may not be explicitly described in the Concept Dictionary. In the case of a collision between entities identified in the two steps, an entity found in the first step takes precedence due to its direct link to the OpenMRS Concept Dictionary. Figure 13 shows the control flow for tagging a document.

Figure 13: Document Tagger control flow

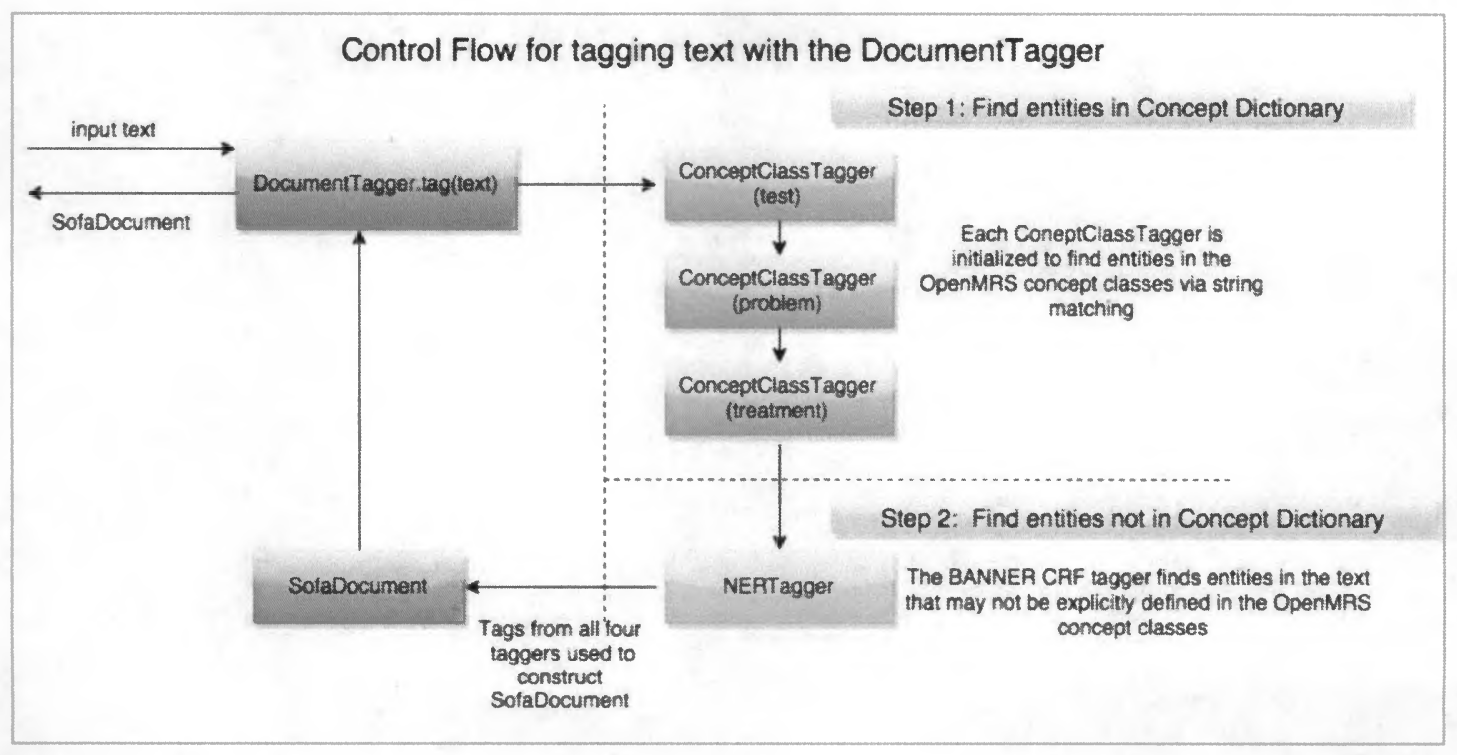

An instance of the DocumentTagger contains the method ".tag()" which takes a String as an argument and returns a fully instantiated SofaDocument object that contains all the annotations found by all four entity taggers. Code Listing 3 shows an example of the DocumentTagger tagging a text and returning a SofaDocument object. This object is then used to print out all the 'problem' entities. 


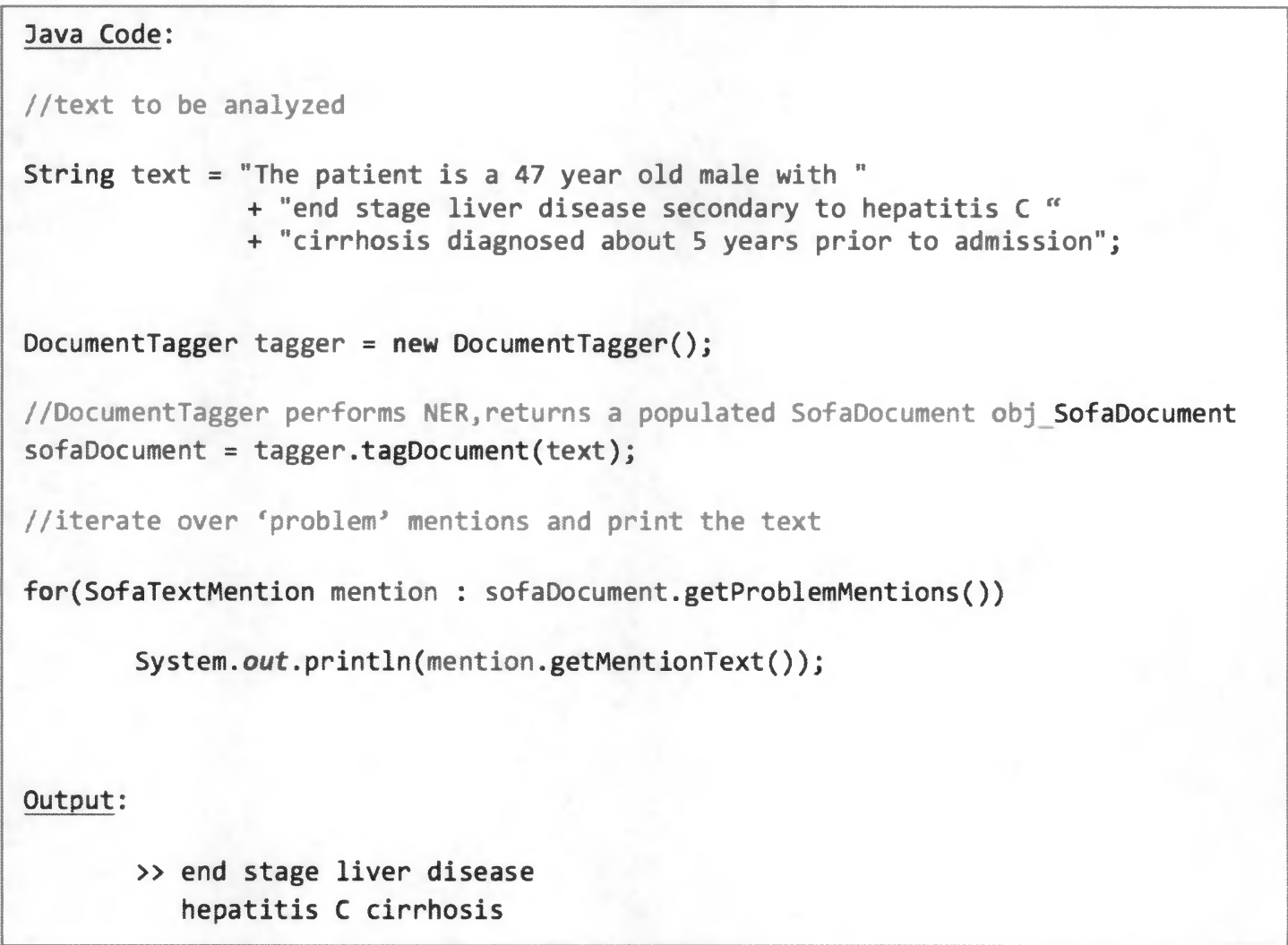

Our module captures and analyzes "Visit Notes," as they are recorded, via the Aspect Oriented Programming (AOP) support provided by the Spring framework [36]. The results of the analysis are persisted in the database for later visualization and retrieval. Figure 14 shows how VisitNoteAdvice uses AOP to interrupt the control flow when the user submits a new visit note. The user completes the Visit Note form and clicks the "save" button. The "save" function uses the OpenMRS EncounterService to save the information in the Encounter. Before the method EncounterService.saveEncounter() is executed, 
control is passed to our VisitNoteAdvice object which analyses the text and stores it in the database before returning the control.

Figure 14: VisitNoteAdvice control flow interrupts the submission of a visit note to perform analysis

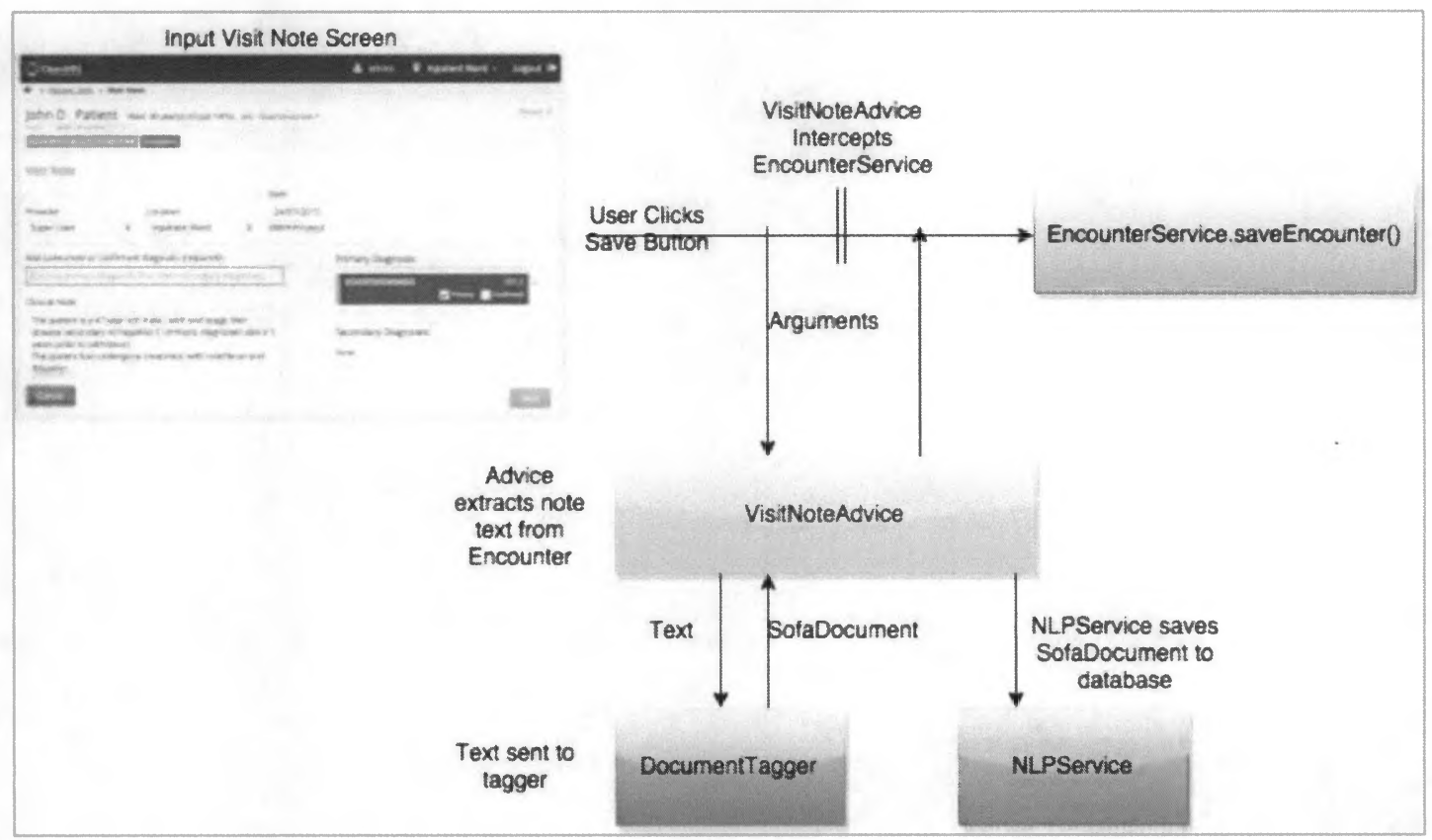

\subsubsection{User Interface}

The module provides an interactive user interface for browsing and visualizing Visit Notes for individual patients and the entities contained in them, see Figure 13. The UI consists of four major components: Word Cloud (top section of figure), Visit NoteEntity List (lower left section), Document Rendering (lower right section), and Search History (just below top section). The Word Cloud presents the most frequently identified entities in the patient's Visit Notes. Words are color coded by Entity Type and font size determined by entity frequency. A mouse click on an entity in the Word Cloud will filter 
the Document-Entity Browser for documents containing that entity, and highlight the entity. The Visit Note-Entity List shows a list of the Visit Notes and the entities contained within them. The Document-Entity Browser also allows the user to filter by Entity Type.

Figure 15: Screenshot from Visit Notes Analysis Module after the user clicks on the word 'Demerol' in the word cloud. A clinical note is rendered with all concepts colored by type.

Word Cloud

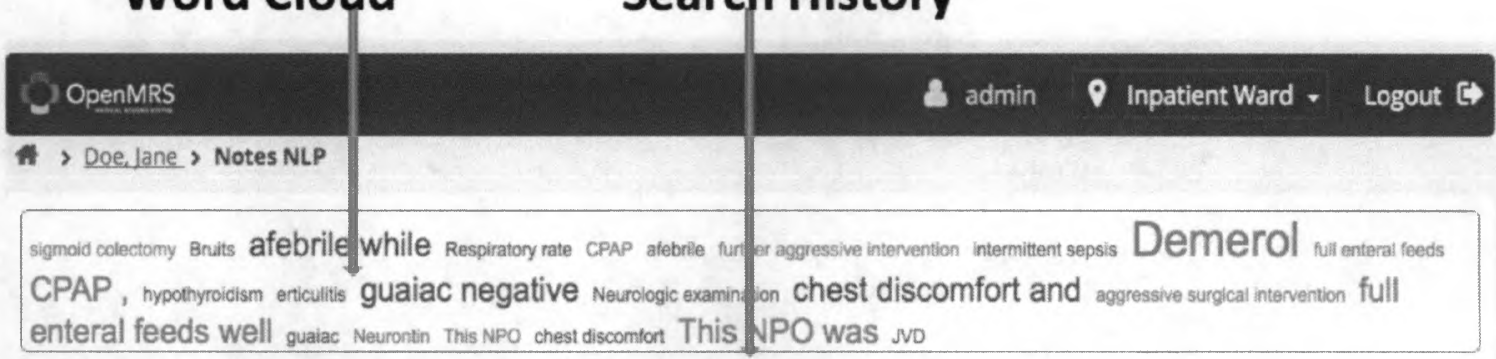

Search History: > afebrile while > chest discomfort and > intermittent sepsis > Demerol

clear history

KEY: test treatment problem

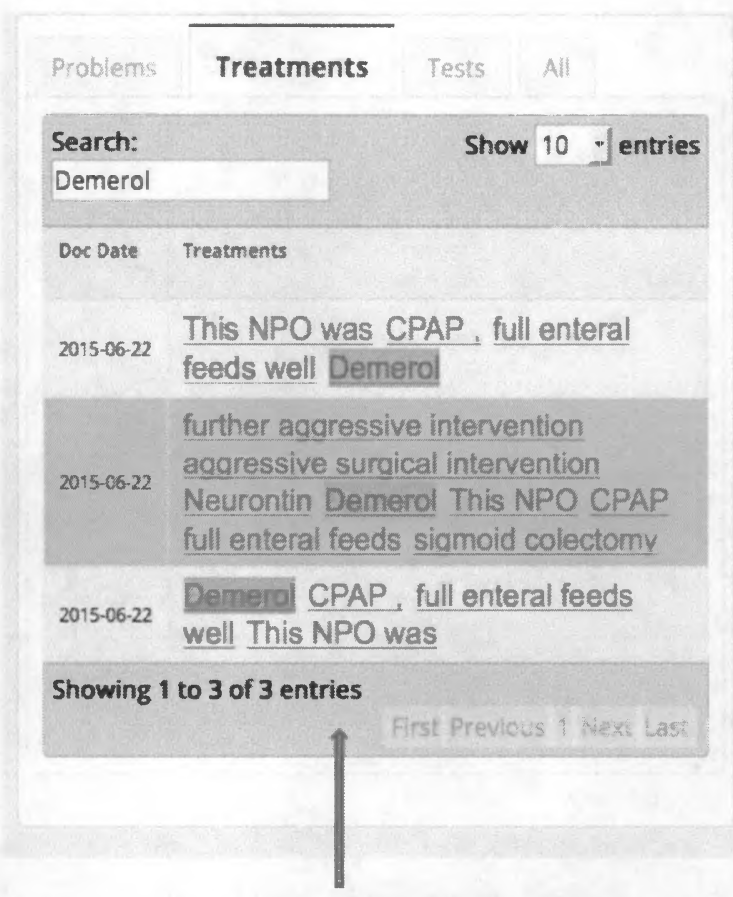

Visit Note - Entity List

\begin{abstract}
DATE: 2015-06-22
Neck: Supple,-JVD, - Bruits

Override added on $12 / 08 / 05$ by :

Sex :

Was most likely due to multiple drugs administered during the episode including Demerol.36-781 Respiratory rate 18

By the end of June, it became clear that Mr.Mass would require aggressive surgical intervention in order to eradicate his intra-abdominal bursts of intermittent sepsis : however, long discussions with the patient and his wife, who was his health-care proxy, revealed that they felt that no further aggressive intervention be attempted. FOLLOW UP APPOINTMENT (S):

****** END OF DISCHARGE ORDERS $* \star * * * *$

HOSPITAL COURSE AND TREATMENT :

SUMMARY OF HOSPITAL COURSE BY SYSTEMS

Admission Date :

Report Status :

The patient had no further e isodes of chest discomfort and remained afebrile whil in house.10.5eizure history on Neurontin .Disp : $\$ 50$ Table $(\mathbf{s}) *$ F She was slow to advance on enteral feeds and gradua / $/$ attained full enteral volume on -.......
\end{abstract}

\section{Document Rendering}

Clicking on an entity in this browser will render the visit note in the Document-

Rendering, highlight the entity of interest, and scroll the note appropriately so that the 
entity is visible. The Navigation History maintains a bread-crumb trail of previous entities examined to quickly retrace the path of the user's navigation history through the data.

One exemplar use case is shown in Figure 13. The user navigates to the module's main page and is presented with the word cloud and the Document-Entity Viewer that shows entities found in the patient's documents (in this case, the "Treatment" tab has been selected to view all treatment entities). The user clicks on "Demerol" in the Word Cloud and all documents containing the entity "Demerol" are shown in the Document-Entity Browser. The user then chooses a document to view by clicking on "Demerol" in the document-entity of interest and the document is rendered in the Document-Rendering, centered on the occurrence of "Demerol." The user can also use the search box to directly search the entity list for a desired entity.

\subsection{Visit Notes Analysis and Trainer Application \\ 5.2.1 Web Application}

The OpenMRS module is distributed with a generic BANNER model trained on the I2B2 corpus. This model showed strong performance when used to tag documents in the I2B2 corpus, but textual and linguistic differences between I2B2 documents and Visit Notes encountered in the field might degrade the performance of the default model. Therefore, we have developed a companion web application that allows the user to train a new BANNER model with Visit Notes recorded and annotated in the OpenMRS module. This Training Application provides an interface for the user to amend and add annotations 
to a Visit Note corpus and then use this newly corrected corpus to train a new BANNER model.

By design, the application sits outside of the OpenMRS system and has only indirect access to the database. The application accesses the visit notes and annotations through an endpoint exposed by the OpenMRS module. Figure 16 displays the data flow between OpenMRS and the Training Application.

Figure 16: Data flow between Visit Notes Analysis module and Visit Note Annotator and Training Application

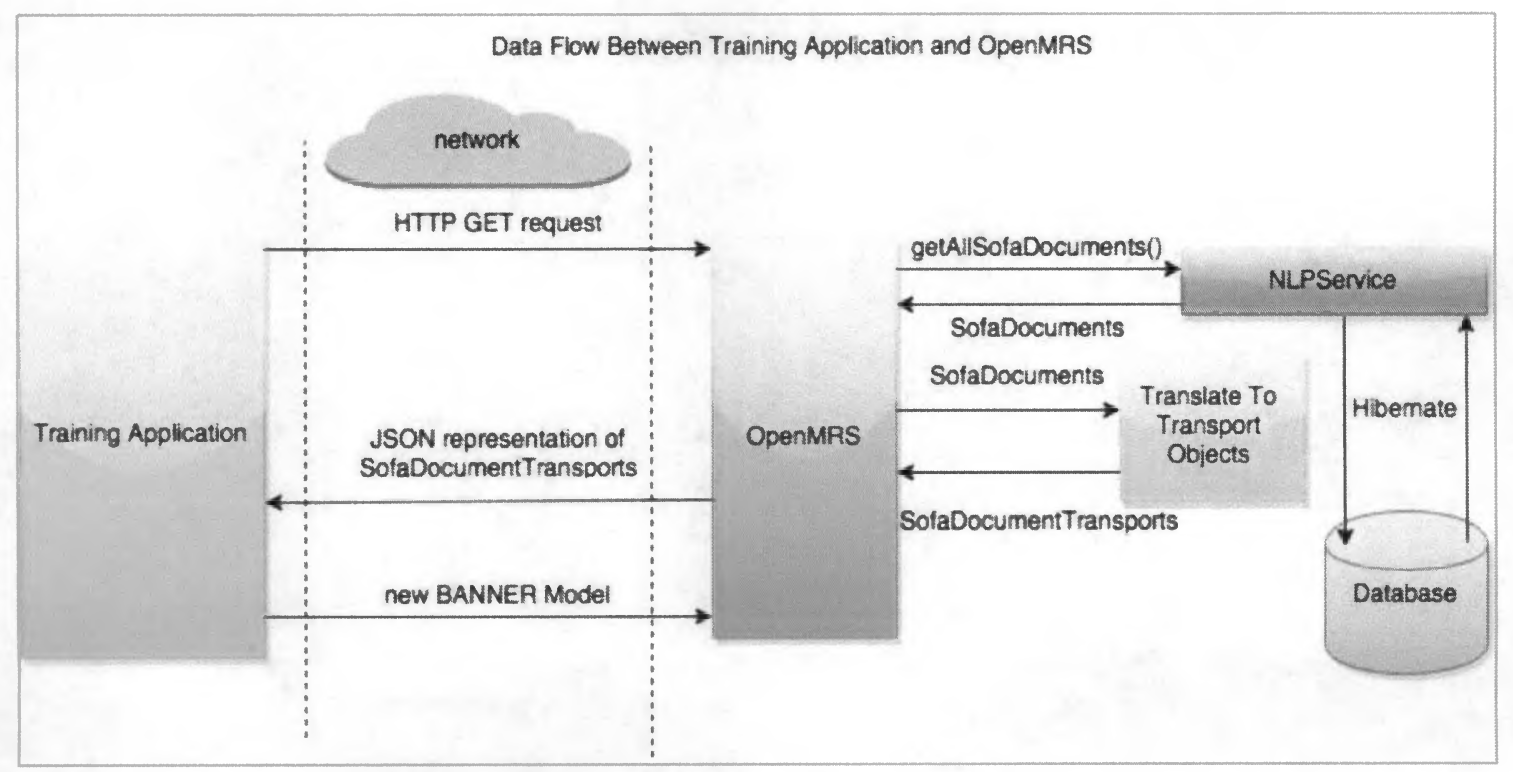

To train a new model, the user navigates to the training web application, figure 17, and provides a URL for the OpenMRS implementation. Our OpenMRS module provides a ReST [38] endpoint to facilitate the transfer of Visit Note data. Visit Notes and corresponding annotations are then communicated to the training application by OpenMRS. The application provides an interface that allows the user to review the 
annotations and correct any mistakes the current model may have made. After the annotations have been corrected, they are provided to BANNER to generate a new model. The new model is then downloaded by the user and subsequently uploaded into the OpenMRS module. The module can maintain several models that can be used interchangeably by the user. Figure 17 shows a screenshot of the application in use.

Figure 17: I'isit Note Annotator and Trainer application

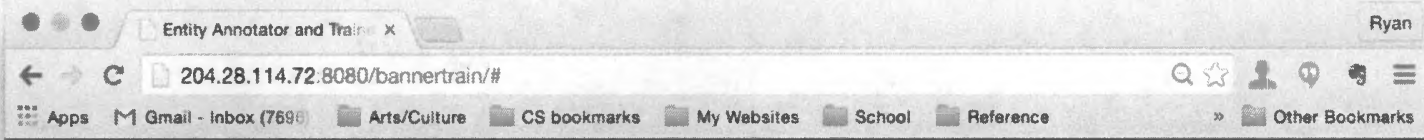

Visit Note List

show 10 : entries

Search:

Docid Date Mame

1. 07/24/2015 Pationt. Jokn 15:08:15

07/26/2015 Patient, John

12:03:42 100-8

07/28/2015 Patient, John

18:33:00 100-8

4 07/28/2015 Patient, John

\begin{tabular}{lll}
\hline & 18.35 .48 & $100-8$ \\
\hline Docid & Date & Name
\end{tabular}

Showing 1 to 4 of 4 entries

Provious 1 Next

Probian Teat Treatment Modified Save Bulld

HISTORY OF PRESENT ILLNESS : The patient is a 47 year old male, with and atage herer disease secondary to hepatitis $\mathrm{C}$ cirnhosis diagnosed about 5 years prior to adrission. The patient had undergone treatment with interferon and Plibavirn. He mad been admitted to the St. Margaret 's Conter for Women \& Intants multiple times eariy in 2013 for menapwinent of encephalopathy and ascites. The patient had been discharged from the St. Margaset is Center for Wornen I Infants on 2013-05-06, but was readmitted on 2013-05-09 when noted to have worsening renal function.

Change Log

Show 10 : ontries Search:

From To

worsening worsening nonal function tanalifunction undo

From To

Showing 1 to 1 of 1 entries

Previous I Nayst 
The application architecture follows the Mediator Model View Controller design pattern. This design pattern has four components. The View is responsible for rendering the data to the user through different components of the User Interface. The Model stores and updates data about the application's state, the controllers manipulate the data, and communication between objects in the application is brokered by a Mediator object. The use of a Mediator has a decoupling effect on the system and reduces dependencies between classes. The Model is a list of SofaDocumentTransport objects, which are a simplification of the SofaDocument object used in the OpenMRS module. There are two views with corresponding controllers. Each view is front-end HTML component that communicate with its corresponding controller via AJAX requests. The Document List view is controlled by the DocumentListController object. The DocumentViewer view is controlled by the DocumentViewerController object. Controllers do not update the Model directly, but do so through the Mediator. Figure 18 illustrates the underlying system architecture. The application is implemented as a Java web application that is deployed in an Apache Tomcat servlet container. 


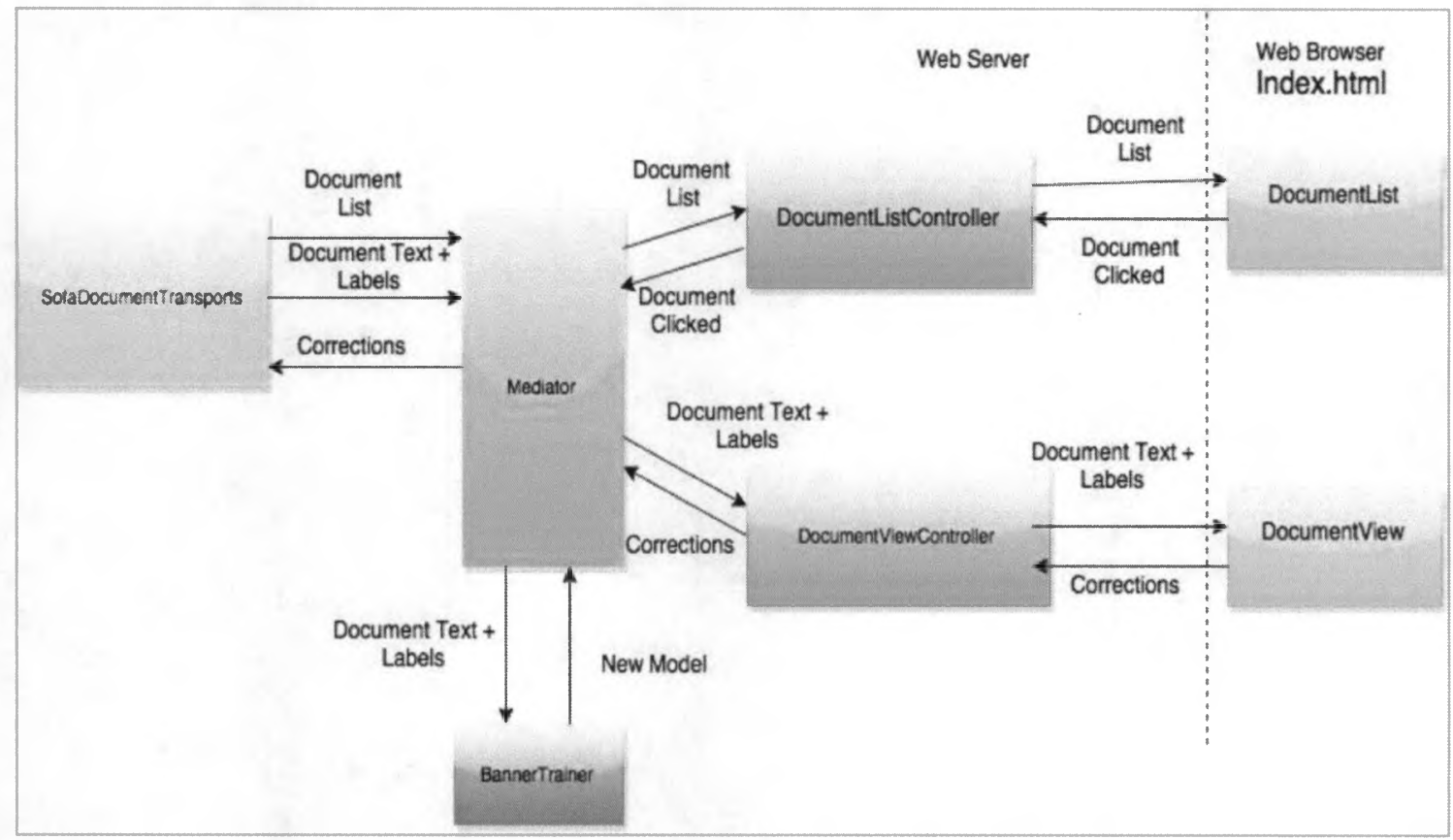

\subsubsection{Example Use Case}

To illustrate how the OpenMRS module and Language Model Trainer application work together, consider the following use case. A user is examining the visit notes for patient John D. Patient. The user notices that one or more words in the visit note are not tagged correctly, figure 19. "Prednisone" is correctly identified as a treatment, but "Neoral", and "CellCept" should have been identified as treatments, but were not, neither was "physical therapy". "liver function tests" should have been recognized as a test, but it was not. 


\section{DATE: 2015-07-26}

He was advanced to a regular house diet later on postop day 3 .The patient was advanced per protocol to an immunosuppressive regimen of prednisone Neoral and CellCept. The patient 's mental status remained essentially clear throughout the entire postoperative period. The patient started ambulating with the assistance of physical therapy following transfer to the surgical floor. At the time of discharge , the patient was independent, ambulating, and functioning well .The patient 's appetite improved significantly, and at the time of discharge the patient was on a regular diet with no tube feed supplements deemed necessary. The patient 's liver function tests all improved appropriately by the time of discharge. The patient 's surgical incision was also healing well by the time of discharge with no evidence of infection .

A user can navigate to the Language Model Training application, load the annotated visit notes, and correct the errors that were observed in the OpenMRS module. In figure 20 , the documents are loaded and we can see that the mistakes match those found in the OpenMRS module. Figure 21 shows the corrections to the annotations made by the user. These corrections are reflected both on the document rendering as well as the Change Log. 


\section{OpenMRS Visit Note Analysis Module Companion}

\section{Visit Note Annotator and Trainer}

\section{host.domain:port populate}

\begin{tabular}{|c|c|c|}
\hline \multirow[b]{2}{*}{ DociD } & \multicolumn{2}{|c|}{10 Search: } \\
\hline & Date & $\begin{array}{l}\text { Name } \\
\text { MAN }\end{array}$ \\
\hline 1 & $\begin{array}{l}07 / 24 / 2015 \\
15: 08: 15\end{array}$ & $\begin{array}{l}\text { Patient, John } \\
100-8\end{array}$ \\
\hline 2 & $\begin{array}{l}07 / 26 / 2015 \\
12: 03: 42\end{array}$ & $\begin{array}{l}\text { Patient, John } \\
100-8\end{array}$ \\
\hline 3 & $\begin{array}{l}07 / 26 / 2015 \\
18: 33: 00\end{array}$ & $\begin{array}{l}\text { Patient, John } \\
100-8\end{array}$ \\
\hline 4 & $\begin{array}{l}07 / 26 / 2015 \\
18: 35: 46\end{array}$ & $\begin{array}{l}\text { Patient, John } \\
100-8\end{array}$ \\
\hline
\end{tabular}

Problem Test Treatment Moditied Save Build

He was advanced to a regular house diet later on postop day 3 . The patient was advanced per protocol to an immunosuppressive regimen of prednisone, Neoral , and CeilCept. The patient 's mental status remained essentially clear throughout the entire postoperative period

The patient started ambulating with the assistance of physical therapy following transfer to the surgical floor. At the time of discharge, the patient was independent, ambulating, and functioning well The patient 's appetite improved significantly, and at the time of discharge the patient was on a regular diet with no tube feed supplements deemed necessary. The patient 's liver function tests all improved appropriately by the time of discharge. The patient 's surgical incision was also healing well by the time of discharge with no evidence of infection
Change Log

Show 10 ; entries Search:

From To

\begin{tabular}{c} 
No data available in table \\
\hline From To \\
Showing 0 to 0 of 0 entries \\
Previous Next \\
\hline
\end{tabular}

Figure 21: Annotations are corrected.

\section{Problem Test Treatment Modified Save Build}

He was advanced to a regular house diet later on postop day 3 .The patient was advanced per protocol to an immunosuppressive regimen of prednisone, Neoral, and CellCept. The patient 's mental status remained essentially clear throughout the entire postoperative period .The patient started ambulating with the assistance of physical therapy following transfer to the surgical floor. At the time of discharge, the patient was independent, ambulating, and functioning well. The patient 's appetite improved significantly, and at the time of discharge the patient was on a regular diet with no tube feed supplements deemed necessary. The patient 's liver function tests all improved appropriately by the time of discharge. The patient 's surgical incision was also healing well by the time of discharge with no evidence of infection

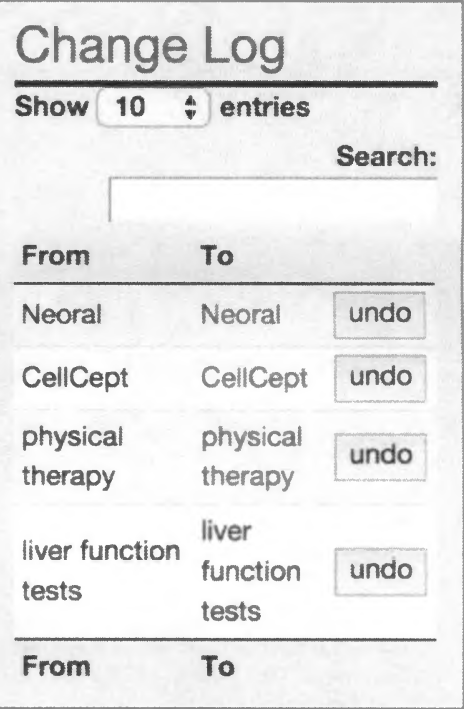


When the user is satisfied with the changes made to the text, they can save the changes by clicking on the save button. Once the user is satisfied with all the annotations of all the documents they select the "Build" button and the text and annotations are used to train a new BANNER Language Model. The user is able to download this model from the training application and upload it to the OpenMRS implementation via a configurations page, figure 22 . 


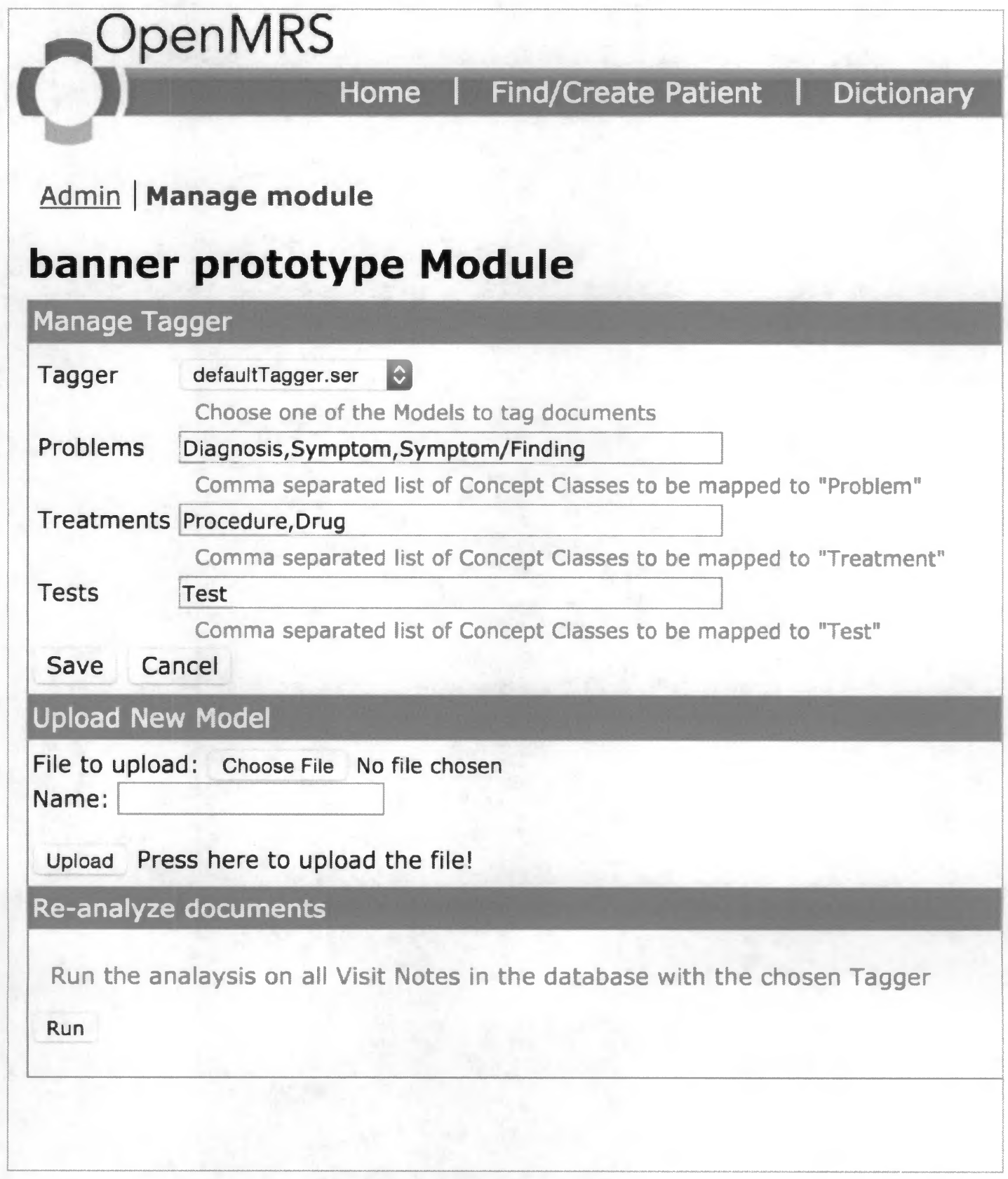

After the user uploads the new model, he or she can now select it for use as the current tagger. This tagger will be used to analyze all subsequent visit notes. The user also has the option to retro-actively apply this new tagger on previously analyzed visit notes. 
This new tagger was able to successfully find the correct entities in the text. Figure 23 shows the results of the new analysis.

Figure 23: Correct annotations with new model.

DATE: 2015-07-26

He was advanced to a regular house diet later on postop day 3 .The patient was advanced per protocol to an immunosuppressive regimen of prednisone , Neoral , and CellCept .The patient 's mental status remained essentially clear throughout the entire postoperative period. The patient started ambulating with the assistance of physical therapy following transfer to the surgical floor .At the time of discharge , the patient was independent, ambulating , and functioning well .The patient 's appetite improved significantly, and at the time of discharge the patient was on a regular diet with no tube feed supplements deemed necessary. The patient's liver function tests all improved appropriately by the time of discharge. The patient 's surgical incision was also healing well by the time of discharge with no evidence of infection .

\subsection{Technical Challenges}

As an Open Source project, OpenMRS suffered from some common problems with open source software. One of the larger hurdles was lack of up-to-date documentation. While many implementation details and common issues are discussed on the public wiki site, subjects are often under-developed or hard to find. However, OpenMRS does have the benefit of enthusiastic developer and implementer communities with active mailing 
lists and chat channels. On several occasions, issues that we ran into that were not easily resolved through the online documentation, were resolved by posting a question to the various forums.

During the implementation of the module and Language Model Trainer application several technical challenges had to be overcome. One challenge was caused by the way Java, Spring, and Maven organize the resource files for an OpenMRS module. Any miscellaneous non-java source file, such as text or configuration files, need to be declared as resources in the Maven pom.xml file. These resources can subsequently be accessed through the ClassPathResource class. This however posed a problem for the BANNER library, which was often required to read from local configuration files while instantiating internal objects. The location of these configuration files are hard coded internally and dependencies could not locate the necessary files. To overcome this issue, we decided to ship our module with two serialized objects. The two objects are a BANNER CRFTagger object and a Lemmatiser object. Both of these classes read from a configuration file during instantiation, which makes them difficult to instantiate within the module itself. Accordingly, we instantiated the objects outside of OpenMRS, serialized the object, and include the serialized object file as a resource within the OpenMRS module. At runtime, the serialized objects are deserialized and read into memory as initialized Java objects.

The Maven templating feature caused minor issues with the serializationdeserialization process. OpenMRS makes use of the Maven templating engine which reads through local resource files and replaces designated tags with pre specified values. 
However, when Maven executes its templating function on binary files, it often makes unnecessary and detrimental changes to the file that end up corrupting it. As a consequence, Maven often corrupted the serialized object files during the template process. The solution to this problem was to use the pom.xml file to explicitly exclude the binary files from being processed by the maven templating engine.

Development of the Language Model Trainer application required us to pay closer attention to characteristics of the Tomcat Servlet Container whose intricacies were handled for us during the OpenMRS module development. Specifically, we had to consider how Tomcat makes use of multi-threading to execute servlet requests. Tomcat was designed to service many clients simultaneous, and to do so, it maintains several server threads to allow multiple HTTP requests to be handled concurrently. While this means faster return time for the client, it requires special care by the developer. Multithreading of servlets means that no two calls to the same servlet are guaranteed to hit the same thread. In our application, the user interface is used to make changes to the underlying data model through the two different controllers. These controllers are implemented as servlets. Any changes made to the data model must not be maintained locally in a servlet because any local changes are not accessible by other threads. This means that data updates must be stored outside of individual server thread.

To solve this problem, we adopted the Mediator Model View Controller design pattern. There is only one instance of the mediator and one instance of the model, however, there can be many instances of each controller, due to the multi-threading of servlets. 
When the user interacts with the view, and makes a change to the underlying model, the change is passed from the view, to the controller. The controller passes the data to the mediator that updates the underlying model. Whenever a controller needs to update the view, it has to request the data from the mediator. This design ensures that the data in the controllers and views are consistent and up to date.

\subsection{Runtime Evaluations}

In order to consider the effect of our module on a running OpenMRS implementation, we examined the execution times and system resource usage under a load of 1000 random clinical notes generated by a random combination of sentences from the I2B2 corpus. Measurements were made on a late 2011 Macbook Pro with a $2.2 \mathrm{GHz}$ intel core 17 processor and $16 \mathrm{~GB}$ of memory and OpenMRS was running out of the Jetty servlet container on java 1.7. Figure 24 shows that the execution time is linear in the number of documents and that in a running OpenMRS system, a document takes under one second to tag. Figures 25 and 26 show CPU usage and Memory consumption respectively. Execution began just after 12:00 and continues through about 12:07 on the X-axis. 
Figure 24: Execution time for tagging documents in a live OpenMRS installation

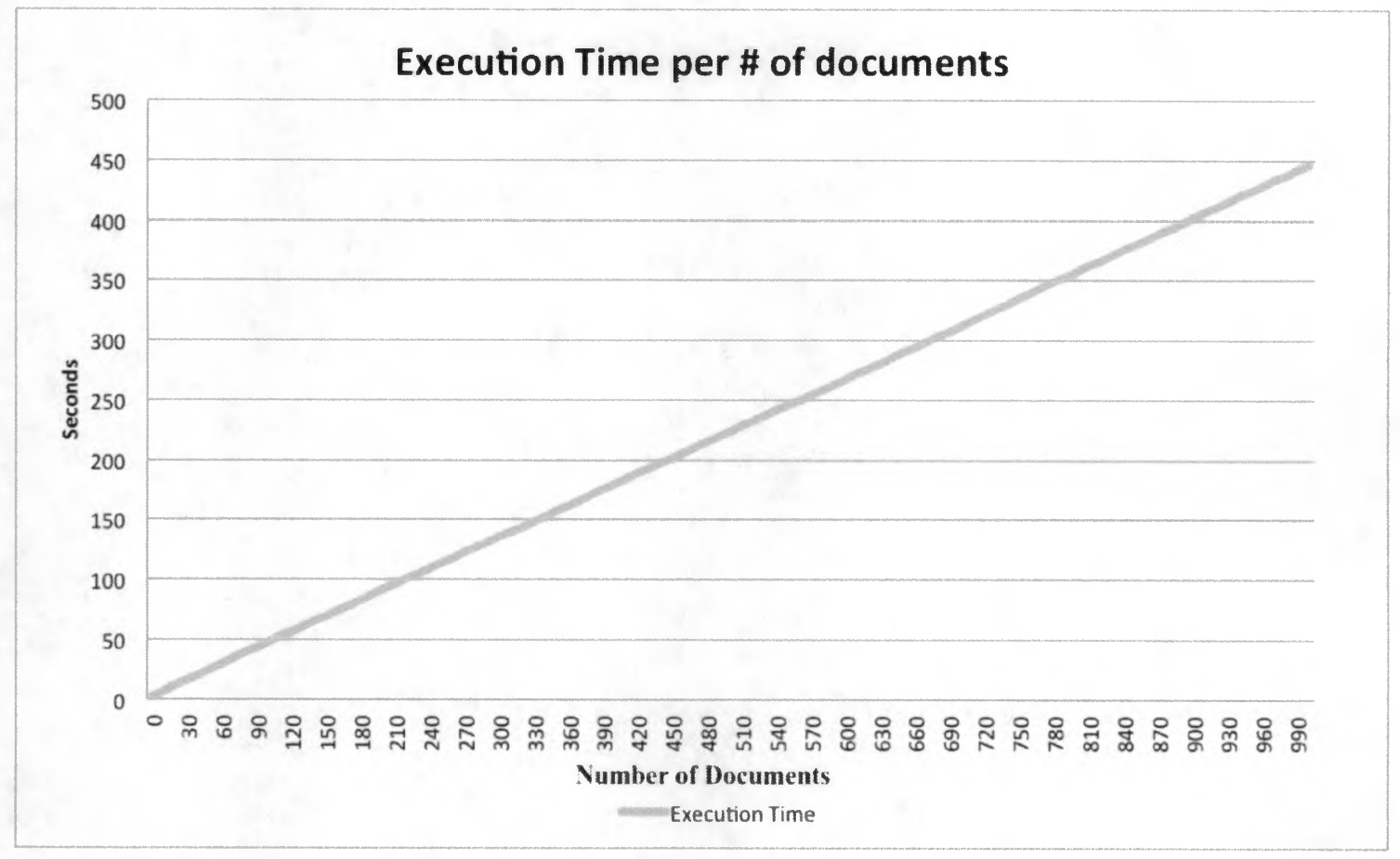

Figure 25: CPU usage of module during document processing.

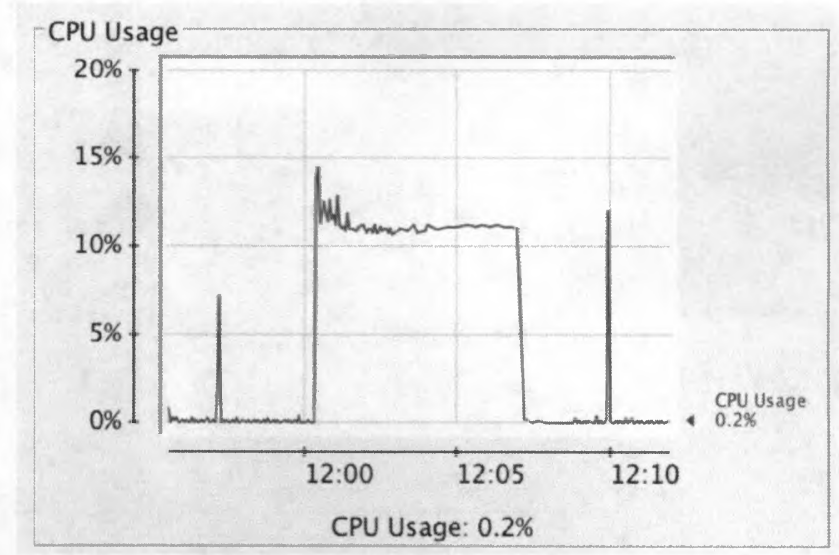


Figure 26: Memory consumption during document processing.

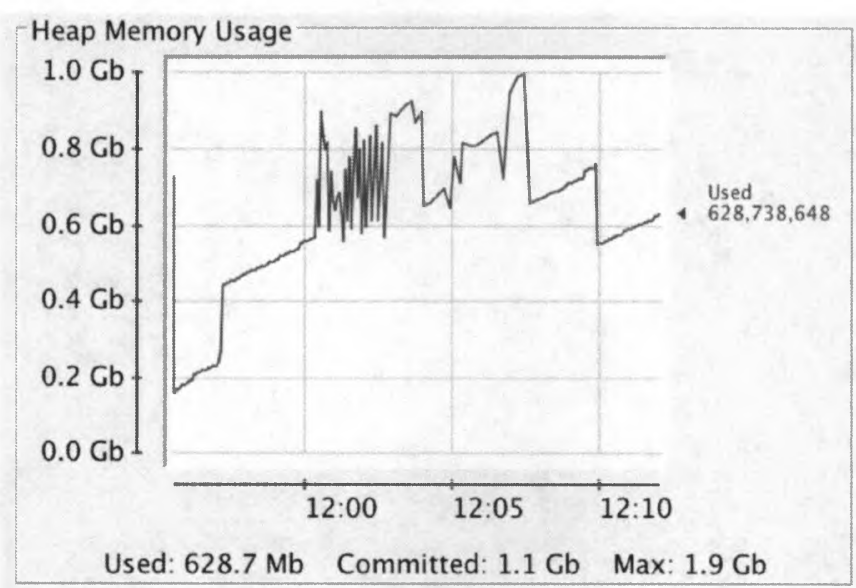

\subsection{Feedback from the Community}

During the development of our module, we reached out to the medical community for feedback and insight into our project. Many clinicians identified the need for quick and succinct patient summaries. One nurse Practitioner responded that

In todays "see a patient every 5 minutes" [atmosphere], I think this would be quite useful for the practitioner to get a quick review of hx [health history] and cc [chief complaint] before entering the room.

Several stakeholders expressed a need for a robust search function to allow a practitioner to quickly find the information of interest. One physician went as far as to say that it can take several hours of research to satisfy an information need about a patient without support for searching a patient's records. With this in mind, we provide the user with a search box that lets them search through the identified entities for a given patient. OpenMRS 2.x 
provides additional support for searching the entire contents of a patient's record with the Chart Search module. 


\section{Chapter 6}

\section{Discussion and Conclusion}

In this concluding chapter we will recap the steps undertaken in order to include Named Entity Recognition technology in the open source EMR OpenMRS. The results of these steps made several limitations clear, both limitations of our own implementation as well as limitations inherent in the state of the art of NLP and NER. We will discuss these limitations, describe future directions and finally provide some concluding remarks.

\subsection{Limitations of the Implementation}

Our tool is built using the open source NER system BANNER and the evaluations that lead up to the decision to use BANNER identified some limitations of the current Biomedical NER systems in general. The best F1 scores that we observed during our evaluations were in the mid $.80 \mathrm{~s}$ ( .84 for single system, .86 for an ensemble system). These scores indicate a good, but not perfect, ability to identify Named Entities within text. We have provided a companion Language Model Training application with the intent of ameliorating this issue and allow the user to tune a BANNER model for better performance at a specific location. However, the performance evaluations clearly show that confidence in the entities identified by the system should not be overstated. The tools that we provide 
should be used to help guide practitioners in their review of visit notes, and is by no means meant to replace the need for thorough review.

In addition to the limitations inherent in the current NER systems we evaluated, our evaluation methods did introduce bias. Our model for the medical language used in practice to write clinical narratives was solely informed by the I2B2 training corpus. This corpus was the basis of our evaluations, and the basis of the training process for BANNER. The evaluations should be trusted to the extent that I2B2 reflects language clinical narratives at large. We were unfortunately unable to obtain sample clinical narratives from other institutions and other locations to compare the I2B2 notes. Some notable characteristics of the I2B2 corpus that will not likely be constant across clinical narratives in the field are: 1) For the most part, I2B2 showed proper use of English grammar which was likely a result of an inherent post-processing step by the transcriptionist. 2) The Notes showed very few typos. 3) Notes came pre-segmented at the sentence level. 4) They showed consistent use of abbreviations 5) They showed consistent document structure with headings between sections. With the exception of possibly the fifth characteristic, differences in clinical notes in the field will likely affect the performance of each NER system. We built the companion trainer application with the hopes of addressing this issue.

An additional limitation is with regard to the User Interface. We have provided a user interface to interact with the results of the NER analysis, but there is much room for the development of a better, more intuitive UI. Our UI can become cluttered when documents with a large number of entities are present. Even with these limitations, the 
Visit Notes Analysis module provides a new and valuable perspective of the content of Visit Notes and has the potential to promote more efficient patient information synthesis.

\subsection{Conclusions}

While there has been steady progress in Natural Language Processing research in the bio-medical domain, efforts to translate these achievements into point of care tools to assist medical practitioners on day-to-day tasks have not kept pace.

With this in mind, we have conducted an extensive empirical evaluation of four Named Entity Recognition systems. These systems include: 1) MetaMap from the US National Library of Medicine. 2) Apache cTAKES, developed originally at the Mayo Clinic. 3) BANNER, developed at Arizona State University, and 4) MGrep from the University of Michigan. We also evaluated ensemble configurations of these systems. Through these evaluations we found that the Conditional Random Fields based NER system BANNER showed the best performance on the I2B2 corpus. These evaluations were necessary in order for us to choose the appropriate NER system and this information was not previously available in the research literature. Therefore, our results will serve to aid future developers and researchers undertaking similar tasks.

Informed by these evaluations, we used BANNER in the development of a Point of Care, patient note summary and navigation tool for the open source EMR, OpenMRS. This module extracts Named Entities that fall within three semantic categories, Problem, 
Treatment, and Test. These entities are then used to provide Visit Note summaries and a tool to assist clinicians in navigating the content of the text. We have also exposed an API for future OpenMRS developers to build on top of our work.

We also developed a companion web application that allows the user to train NER models on new data recorded in OpenMRS, thus making our system flexible and adaptable to language usage in different locations.

To conclude, we present this OpenMRS Visit Notes Analysis Module and API, and the Visit Notes Annotator and Training application as well as the NER system evaluations undertaken to support them, as at once, a set of tools to extract real information value from clinical narratives as well as a step towards bridging the gap between the output of the research community and the needs of practitioners in the field.

\subsection{Future Directions}

There are many directions this project could take when we receive more feedback from end users in the field. This will require users to engage and start interacting with the technology so they that they can further identify weaknesses and new possible features. One example direction, that was mentioned in the limitation section, is the need for a more intuitive User Interface. Enhancements of this type would be a result of observing how people are using the system and what kinds of insights they find most valuable. 
Additionally, we have provided support for some rudimentary reporting, such as a data dump of notes and annotations, as well as a report on the frequency of each entity identified. This functionality will give the users an idea of the reporting capabilities and data available in the system. As reporting requirements become more clear, the reporting mechanism can be enhanced to satisfy them.

We have exposed the underlying API in OpenMRS that carries out the NER functions of the module. We hope that future developers will see new and unexpected uses for the NER capabilities that we have provided to the OpenMRS ecosystem and that the API will provide the support for developers to implement their new ideas. 


\section{Bibliography}

[1] C. Moore, "Diving into Data,"InfoWorld (October 25,2002), http://www.infoworld.com/article/02/10/25/ 021028feundata_1.html.

[2] Singh H, Spitzmueller C, Petersen NJ, et al. Information overload and missed test results in electronic health record- based settings JAMA Intern Med. 2013;173:702-704.

[3] Hirsch, Jamie S., et al. "HARVEST, a longitudinal patient record summarizer." Journal of the American Medical Informatics Association $22.2(2015): 263-274$.

[4] http://openmrs.org/

[5] Savova, Guergana K., et al. "Mayo clinical Text Analysis and Knowledge Extraction System (cTAKES): architecture, component evaluation and applications." Journal of the American Medical Informatics Association 17.5 (2010): 507-513

[6] Aronson, Alan R., and François-Michel Lang. "An overview of MetaMap: historical perspective and recent advances." Journal of the American Medical Informatics Association 17.3 (2010): 229-236.

[7] Settles, Burr. "ABNER: an open source tool for automatically tagging genes, proteins and other entity names in text." Bioinformatics 21.14 (2005): 3191-3192.

[8] McCallum, Andrew K. "\{MALLET: A Machine Learning for Language Toolkit $\}. "$ (2002).

[9] Kim,J. et al. (2003) GENIA corpus - a semantically annotated corpus for bio-textmining. Bioinformatics, 19(Suppl. 1), i180-i182.

[10] Yeh,A., Hirschman,L., Morgan,A. and Colosimo,M. (2004) Task 1A: gene-related name mention finding evaluation. In Proceedings of the Critical Assessment of Information Extraction Systems in Biology (BioCreAtIvE) Workshop, Grenada, Spain.

[11] Leaman, Robert, and Graciela Gonzalez. "BANNER: an executable survey of advances in biomedical named entity recognition." Pacific Symposium on Biocomputing. Vol. 13. 2008.

[12] Dai, M., N. H. Shah, and W. Xuan. "An efficient solution for mapping free text to ontology terms. AMIA Summit on Translational Bioinformatics." San Francisco CA (2008).

[13] Uzuner Ö., Goldstein I, Luo Y, Kohane I. "Identifying patient smoking status from medical discharge records". $J$ Am Med Inform Assoc. 2008; 15(1)15-24.www.jamia.org/cgi/content/short/15/1/14.

[14] Bada, M., Eckert, M., Evans, D., Garcia, K., Shipley, K., Sitnikov, D., Baumgartner Jr., W. A., Cohen, K. B., Verspoor, K., Blake, J. A., and Hunter, L. E. Concept Annotation in the CRAFT Corpus. BMC Bioinformatics. 2012 Jul 9;13:161. doi: 10.1186/14712105-13-161. [PubMed:22776079]

[15] https://wiki.openmrs.org/display/docs/Concept+Dictionary+Basics

[16] http://www. who.int/classifications/icd/en/

[17] http://www.nlm.nih.gov/research/umls/Snomed/snomed main.html

[18] http://www.nlm.nih.gov/research/umls/rxnorm/

[19] http://www.osehra.org/content/distributions-vista

[20] http://sourceforge.net/projects/mumps/

[21] http://www.open-emr.org/

[22] Prokosch HU, McDonald CJ. The effect of computer reminders on the quality of care and resource use. In: Prokosch HU, Dudeck J, editors. Hospital information systems: design and development characteristics; impact and future architecture. Elsevier Science; 1995. p. $221-240$ 
[23] Hirsch, Jamie S., et al. "HARVEST, a longitudinal patient record summarizer."Journal of the American Medical Informatics Association 22.2 (2015): 263-274.

[24] Demner-Fushman, Dina, Wendy W. Chapman, and Clement J. McDonald. "What Can Natural Language Processing Do for Clinical Decision Support?"Journal of biomedical informatics 42.5 (2009): 760-772. PMC. Web. 29 June 2015.

[25] Haug PJ, Christensen L, Gundersen M, Clemons B, Koehler S, Bauer K. A natural language parsing system for encoding admitting diagnoses. Proc AMIA Annu Fall Symp 1997:814-8.

[26] Friedlin FJ, McDonald CJ. A software tool for removing patient identifying information from clinical documents. J Am Med Inform Assoc 2008;15(5):601-10.

[27] Lafferty, J., McCallum, A., Pereira, F. (2001). "Conditional random fields: Probabilistic models for seamenting and labeling sequence data". Proc. 18th International Conf. on Machine Learning. Morgan Kaufmann. pp. 282-289.

[28] Ferrucci, David, and Adam Lally. "UIMA: an architectural approach to unstructured information processing in the corporate research environment."Natural Language Engineering 10.3-4 (2004): 327-348.

[29] https://gate.ac.uk/

[30] http://nlp.stanford.edu/software/CRF-NER.shtml

[31] http://opennlp.apache.org/

[32] http://mallet.cs.umass.edu/

[33] https://atlas.openmrs.org/

[34] Krovetz, Robert. "Viewing morphology as an inference process." Proceedings of the 16th annual international ACM SIGIR conference on Research and development in information retrieval. ACM, 1993.

[35] Porter, Martin F. "An algorithm for suffix stripping." Program 14.3 (1980): 130-137.

[36] http://www.ncbi.nlm.nih.gov/books/NBK9680/\#ch06.I68 The SPECIALIST Lexical Tools

[37] Cutting D, Kupiec J, Pedersen J and Sibun P. A practical part-of-speech tagger. Proc Third Conference on Applied Natural Language Processing, 1992.

[38] Zhou, X.; X. Zhang; and X. Hu. (2007) Dragon Toolkit: Incorporating Auto-learned Semantic Knowledge into Large-Scale Text Retrieval and Mining. Proceedings of the 19 IEEE International Conference on Tools with Artificial Intelligence (ICTAI).

[39] Shah, Nigam H., et al. "Comparison of concept recognizers for building the Open Biomedical Annotator." BMC bioinformatics 10.Suppl 9 (2009): S14. APA

[40] http://www.ncbi.nlm.nih.gov/pmc/tools/openftlist/

[41] Miller, Eric. "An introduction to the resource description framework." Bulletin of the American Society for Information Science and Technology 25.1 (1998): 15-19.

[42] Cunningham, Hamish. "GATE, a general architecture for text engineering." Computers and the Humanities 36.2 (2002): $223-254$.

[43] http://www.epic.com/

[44] http://www.practicefusion.com

[45] Lovins, Julie B. Development of a stemming algorithm. MIT Information Processing Group, Electronic Systems Laboratory, 1968.

[46] Liu, Haibin, et al. "BioLemmatizer: a lemmatization tool for morphological processing of biomedical text." J. Biomedical Semantics 3.3 (2012): 17.

[47] UMLS® Reference Manual [Internet]. Bethesda (MD): National Library of Medicine (US); 2009 Sep-. 2, Metathesaurus. Available from: http:/www.ncbi.nlm.nih.gov/books/NBK9684/ 
[48] Reynar, Jeffrey C., and Adwait Ratnaparkhi. "A maximum entropy approach to identifying sentence boundaries." Proceedings of the fifth conference on Applied natural language processing. Association for Computational Linguistics, 1997.

[49] Hall, Amanda, and Graham Walton. "Information overload within the health care system: a literature review." Health Information \& Libraries Journal 21.2 (2004): 102-108.

[50] Wrenn, Jesse O., et al. "Quantifying clinical narrative redundancy in an electronic health record." Journal of the American Medical Informatics Association 17.1 (2010): 49-53. 
Appendix 1:

Visit Notes Analysis Module

User's Guide 

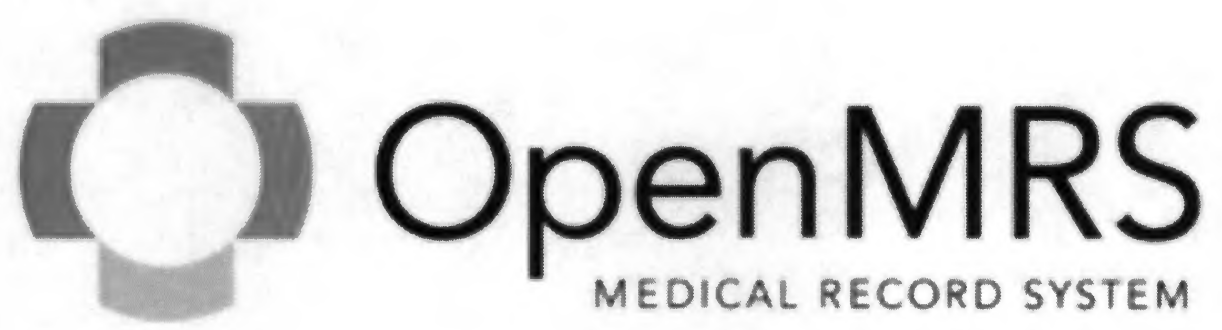

\title{
Visit Notes Analysis Module
}

\section{User's Guide}

\author{
Ryan Eshleman
}

Advisors: Dr. Barry Levine 


\section{Dr. Hui Yang}

Table of Contents:

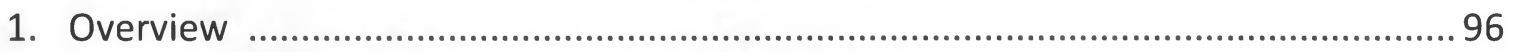

1.1. Named Entity Recognition of Visit Notes ........................................................... 96

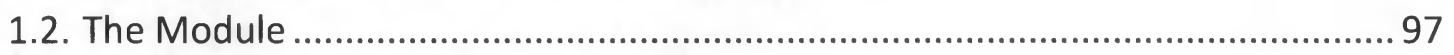

1.3. The Named Entity Recognition Algorithm .......................................................... 98

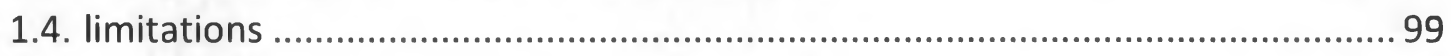

2. Visit Note Analysis User Interface ...................................................................... 100

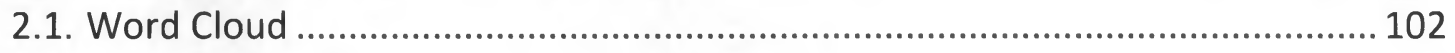

2.2. Search History .................................................................................................. 104

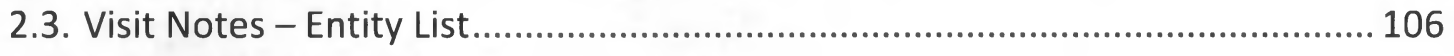

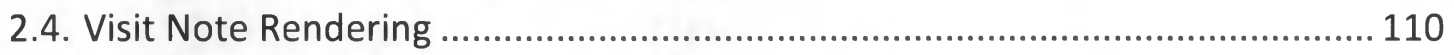

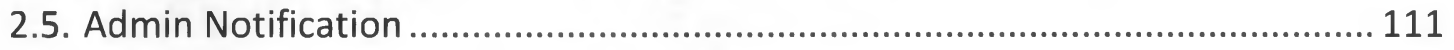




\section{Overview}

"Text search is only useful if you already know what you are looking for."

- Ancient Proverb

1.1 Named Entity Recognition of Visit Notes.

Figure 1: Sample Visit Note with Named Entities recognized

The patient is a 47 year old male, with end stage liver disease secondary to hepatitis $C$ cirrhosis diagnosed about 5 years prior to admission. The patient had undergone treatment with interferon and Ribavirin . He had been admitted to the St.Margaret 's Center for Women \& Infants multiple times early in 2013 for management of encephalopathy and ascites. The patient had been discharged from the St.Margaret 's Center for Women \& Infants on 2013-05-06, but was readmitted on 2013-05-09 when noted to have worsening renal function.

OpenMRS and Electronic Medical Record Systems in general tend to be great at processing structured data, but they often struggle to provide adequate support for processing and analysis of unstructured data, such as text recorded in Visit Notes. OpenMRS is ahead of the curve with its Chart Search module that allows 
the user to perform text search over all the patient data, including Visit Notes. A problem however, is that text search is only useful if the user knows in advance what they are looking for.

This module is designed to tag and extract salient information from Visit Notes. This information is in the form of Named Entities, which can be understood as element of the text that fall within pre-defined conceptual categories. Named Entities are defined at a higher level of abstraction than syntactic elements such as noun, verb, and adjective. In the sentence "The man walked into Starbucks Coffee," while both "man" and "Starbucks Coffee" are nouns, only the latter would be correctly identified as a Named Entity, potentially of the category "Place." With this definition in mind, the module is designed to recognize bio-medical entities that fall within one of the following three categories: Problem, Treatment, and Test. Figure 1 shows an example of problems and treatments identified by our module.

\subsection{The Module}

This module is designed to recognize bio-medical entities that fall within one of the following three categories: Problem, Treatment, and Test. It recognizes these entities in Visit Notes as they are being recorded by the clinician. After these entities have been identified, they are used to provide snapshot summaries of the information held within the larger text. This allows a practitioner to quickly summarize the content of the text and locate the information of interest. As a result, this can allow the practitioner to spend more time on other tasks, instead of reviewing patient histories. Figure 2 shows the user interface that allows for navigation of Entities within Visit Notes. A breakdown of the UI components is found in section 2 . 
Figure 2: Visit Notes Analysis UI

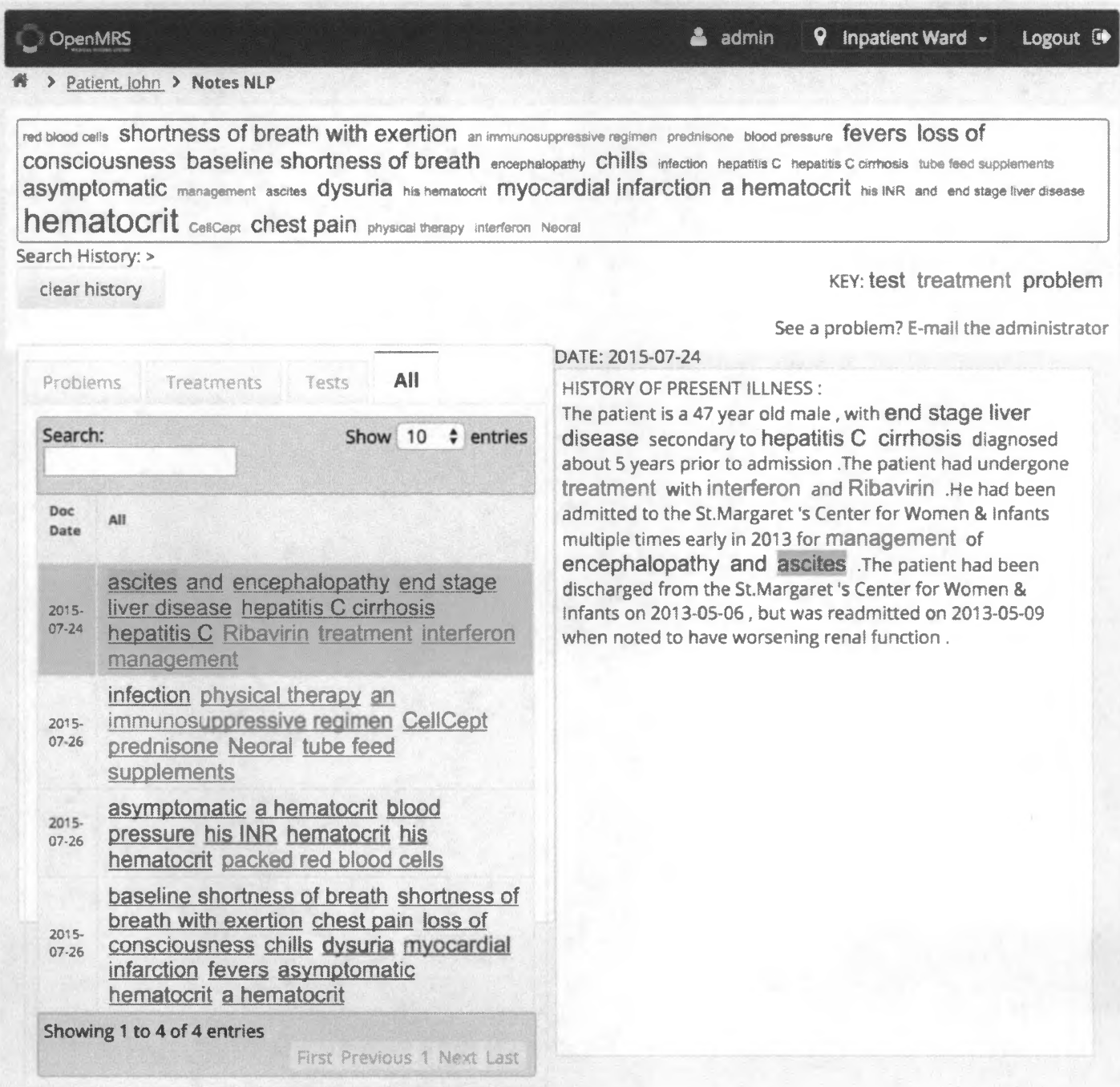

\subsection{The Named Entity Recognition Algorithm}

In our module, Named Entity Recognition is carried out in two steps. The first step uses the concept dictionary present in the OpenMRS implementation to identify spans of text in the visit note that match concepts. The module uses all names and synonyms associated with a concept to perform matching. This means that the module has support 
for identifying concepts in any language whose synonyms are recorded in the Concept Dictionary. This first step is performed by a simple String matching algorithm. The second step is agnostic of the local Concept Dictionary and uses the machine learning algorithm Conditional Random Fields (CRF) to find spans of text that represent Named Entities, but may not have matched a Concept. For example, if a new medicine mentioned in a Visit Note that does not yet have an entry in the Concept Dictionary, then the CRF may identify it as a treatment.

\subsection{Limitations}

During the development of this module, we performed evaluations of several NER algorithms in order to choose the best one to use here. During these evaluations it became clear that the current state of NER is not perfect. This module uses the BANNER NER system and our measurements showed that of the entities it finds, about $80 \%$ are correct. Also, it tends to find about $70 \%$ of the entities in the text. While this performance is good, it indicates that the results of this module should not be relied upon for perfect accuracy, and should be used as a tool to assist in analyzing Visit Notes. 


\section{Visit Note Analysis User Interface}

To navigate to the Visit Note Analysis Interface, go to the patient dashboard for the desired patient. The interface is accessible through the General Actions list as "Visit Notes Analysis." Click this item to get to the interface for Visit Notes associated with this patient. Figure 3 shows the location of the Action on the Patient Dashboard

Figure 3: Finding the Module:

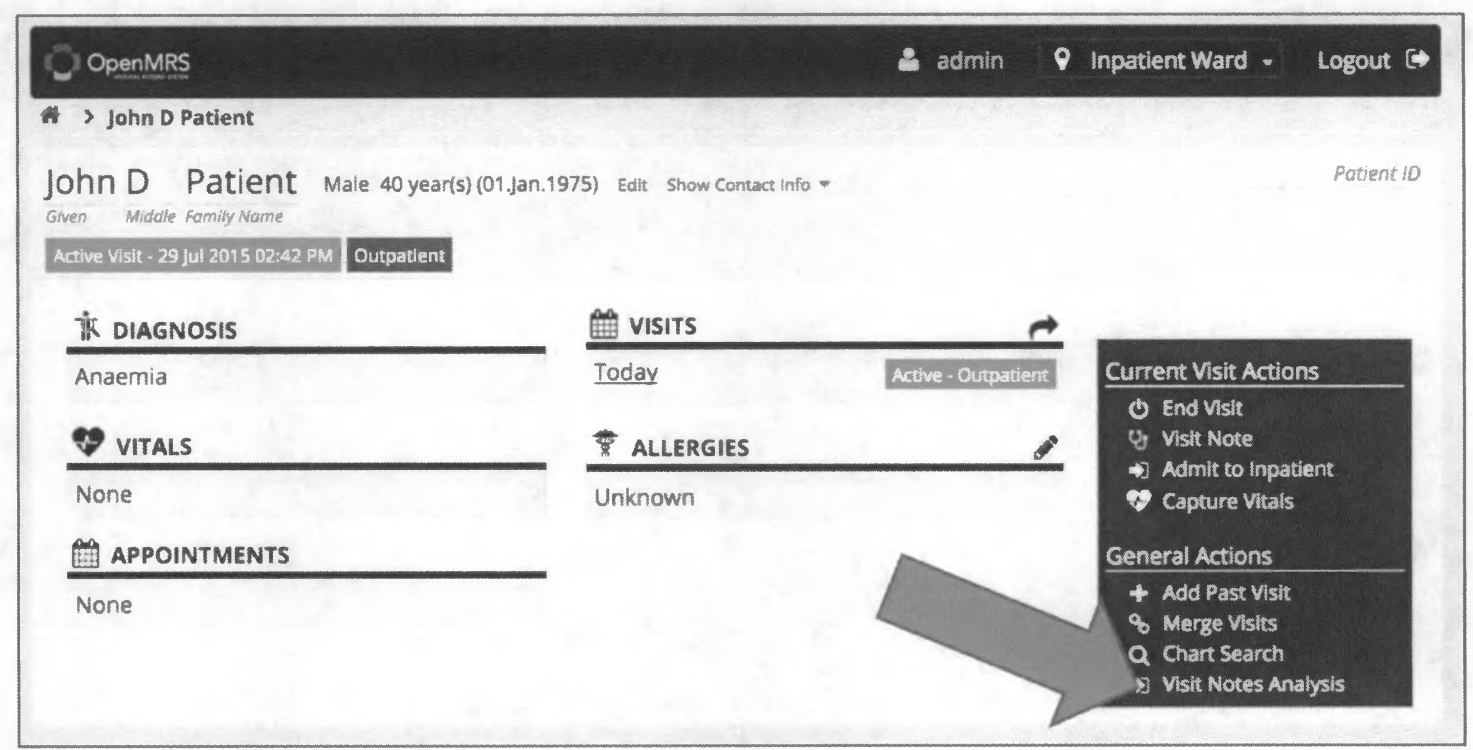

The user interface is broken down into Four major components as labeled on Figure 4
1. Word Cloud
2. Search History
3. Visit Note List
4. Visit Note Rendering
5. Admin Notification 


\section{Search History 1. Word Cloud} Notification

\begin{tabular}{|c|c|c|c|}
\hline OpenMRS & & 9 admin 9 Inpatient Ward & Logout C\$ \\
\hline * > Patient, lohn > No & 5 NLP & & \\
\hline $\begin{array}{l}\text { red blood cells shortnes: } \\
\text { consciousness ba } \\
\text { asymptomatic mana } \\
\text { hematocrit cell }\end{array}$ & $\begin{array}{l}\text { of breath with exertion an immunosuppol } \\
\text { eline shortness of breath encophaiope } \\
\text { nent ascites dysuria his hematocrit myoca } \\
\text { chest pain physicat therapy interferon }\end{array}$ & $\begin{array}{l}\text { sive regimen prednisone blood pressure fevers loss } \\
\text { chills infection hepatitis } \mathrm{C} \text { hepatitis } \mathrm{C} \text { cirnosis twbe fee } \\
\text { lial infarction a hematocrit his INR and end }\end{array}$ & $\begin{array}{l}\text { piements } \\
\text { o liver disease }\end{array}$ \\
\hline Search History: > & $\zeta$ & & \\
\hline
\end{tabular}

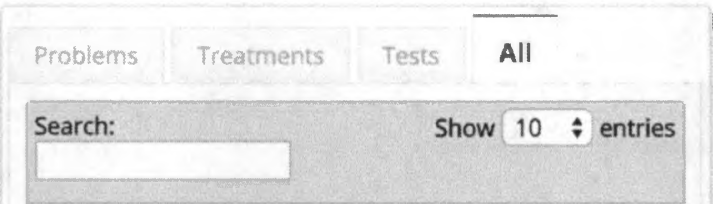

Doc All
Date

ascites and encephalopathy end stage

2015. liver disease hepatitis $\mathrm{C}$ cirrhosis

07.24 hepatitis $C$ Ribavirin treatment interferon management

infection physical therapy an

2015. immunosuppressive reaimen CellCept

07-26 prednisone Neoral tube feed supplements

2015. asymptomatic a hematocrit blood

2015. pressure his INR hematocrit his hematocrit packed red blood cells

baseline shortness of breath shortness of breath with exertion chest pain loss of

2015. consciousness chills dvsuria myocardial infarction fevers asymptomatic hematocrit a hematocrit

Showing 1 to 4 of 4 entries

DATE: 2015-07-24 HISTORY OF PRESENT ILLNESS :

The patient is a 47 year old male, with end stage liver disease secondary to hepatitis $\mathrm{C}$ cirrhosis diagnosed about 5 years prior to admission. The patient had undergone treatment with interferon and Ribavirin. He had been admitted to the St.Margaret 's Center for Women \& Infants multiple times early in 2013 for management of encephalopathy and ascites. The patient had been discharged from the St.Margaret 's Center for Women \& Infants on 2013-05-06, but was readmitted on 2013-05-09 when noted to have worsening renal function.

\section{Visit Note - Entity List}




\subsection{Word Cloud}

The word cloud shows a visualization of the top 30 most frequent entities that appear in the Visit Notes associated with the patient. The relative size of each entity corresponds to the relative frequency of the entity. An entity with a large font size appears more frequently than an entity with a smaller font size. Figure 5 shows an example word cloud.

Figure 5: Word Cloud

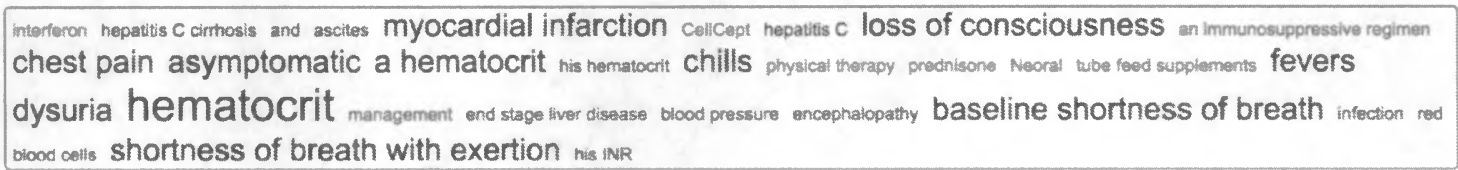

Words are color coded by entity type. Problems in red, treatments in green, and tests in blue. The word cloud is interactive, When an entity is clicked on, the Visit Notes shown in the Visit Notes List are filtered for notes that contain that selected entity. For example, figure 6 shows the document list after "hematocrit" is clicked on in the Word Cloud. The 2 documents containing the entity "hematocrit" are shown. Selecting an item from the Word Cloud also adds an element to the Search History. This is shown in figure 7 below. 
Figure 6: Visit Note - Entity List after "hematocrit" selected in word cloud

\begin{tabular}{|c|c|c|c|c|c|}
\hline \multicolumn{2}{|c|}{ Problems } & Treatments & Tests & All & \\
\hline \multicolumn{3}{|c|}{ Search: } & Show & 10 & * entries \\
\hline \multicolumn{6}{|c|}{ hematocrit } \\
\hline $\begin{array}{l}\text { Doc } \\
\text { Date }\end{array}$ & \multicolumn{5}{|c|}{ Tests } \\
\hline $\begin{array}{l}2015- \\
07-26\end{array}$ & \multicolumn{5}{|c|}{$\begin{array}{l}\frac{\text { red blood cells }}{\text { blood pressure }} \\
\text { hematocrit }\end{array}$} \\
\hline $\begin{array}{l}2015- \\
07-26\end{array}$ & \multicolumn{5}{|c|}{ hematocrit a hematocrit } \\
\hline \multicolumn{6}{|c|}{$\begin{array}{l}\text { Showing } 1 \text { to } 2 \text { of } 2 \text { entries } \\
\text { (filtered from } 4 \text { total entries) }\end{array}$} \\
\hline & & & \multicolumn{3}{|c|}{ First Previous 1 Next Last } \\
\hline
\end{tabular}




\subsection{Search History}

The search history provides a breadcrumb of the entities searched by the user, allowing them to retrace their cognitive steps. In the above example, when the user selected "hematrocrit" from the word cloud, "hematocrit" was also appended to the end of the search history as the below figure shows.

Figure 7: Search History after hematocrit selected

\begin{tabular}{|c|c|}
\hline $\begin{array}{l}\text { interferon hepattis } \mathrm{C} \text { cirnosis and ascites myocardial infarction calcept hepatits c loss of conscious } \\
\text { chest pain asymptomatic a hematocrit his hematocrit chills physical therapy prednisone Neoral tubo } \\
\text { dysuria hematocrit management and stage liver disease blood pressure encephatopathy baseline shortn. } \\
\text { blood cells shortness of breath with exertion his INR }\end{array}$ & $\begin{array}{l}\text { immunosuppressive regimen } \\
\text { ements fevers }\end{array}$ \\
\hline \multicolumn{2}{|c|}{ Search History: > hepatitis C cirrhosis > encephalopathy > end stage liver disease > encephalopathy > ascites > hematocrit } \\
\hline clear history & $\begin{array}{r}\text { KEY: test treatment } \\
\text { problem }\end{array}$ \\
\hline
\end{tabular}

When the user clicks on "hematocrit" in the Search History, the Visit Notes List is filtered for Visit Notes that contain "hematocrit" just as after selecting hematocrit from the word cloud. Figure 6 above shows these results.

An Entity is also added to the "Search History" if it is searched for in the Entity Search Box part of the Visit Note List or if an entity is clicked on in the entity list. More details on the Entity Search Box and entity list are found in the next section.

The user can clear the search history by clicking on the "clear history" button. 
Figure 8: The user has clicked the "clear history" button, and the search history breadcrumb has been reset.

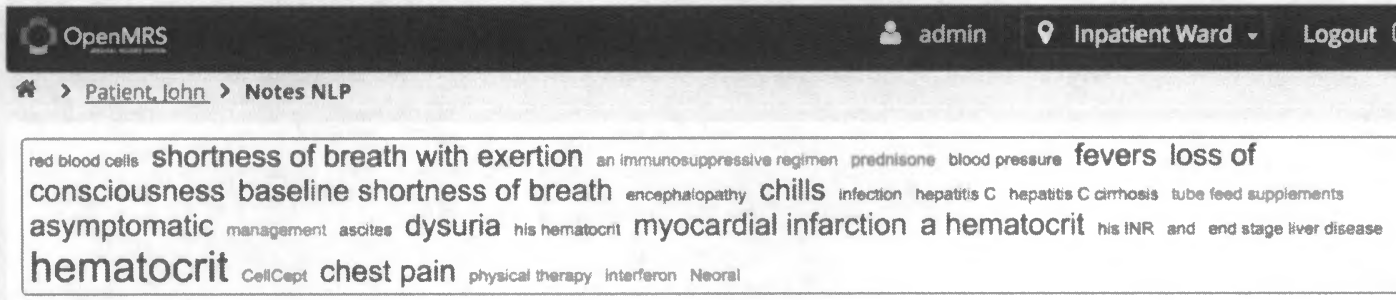

Search History: >

clear history

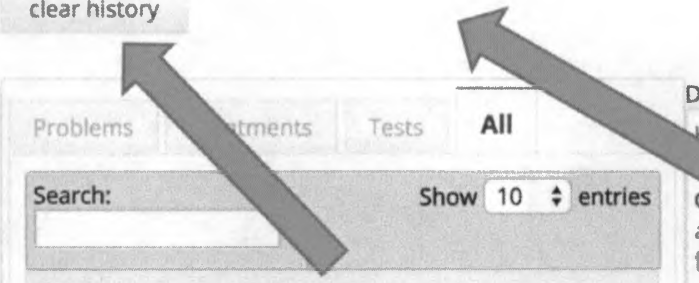

Dot All

ascites and encephalopathy end stage

2015. liver disease hepatitis $\mathrm{C}$ cirrhosis

07.24 hepatitis C Ribavirin treatment interferon management

infection physical therapy an

2015. immunosuppressive regimen CellCept

07-26 prednisone Neoral tube feed supplements

2015.

asymptomatic a hematocrit blood

$07-26$

hematocrit packed

baseline shortness of breath shortness of breath with exertion chest pain loss of

$2015-$
$07-26$ consciousness chills dysuria myocardial infarction fevers asymptomatic hematocrit a hematocrit

Showing 1 to 4 of 4 entries
KEY: test treatment problem

See a problem? E-mail the administrator DATE: $2015-07-24$ HISTORY OF PRESENT ILLNESS :

e patient is a 47 year old male, with end stage liver disease secondary to hepatitis $C$ cirrhosis diagnosed about 5 years prior to admission. The patient had undergone treatment with interferon and Ribavirin. He had been admitted to the St.Margaret 's Center for Women \& Infants multiple times early in 2013 for management of encephalopathy and ascites. The patient had been discharged from the St.Margaret 's Center for Women \& Infants on 2013-05-06, but was readmitted on 2013-05-09 when noted to have worsening renal function. 


\subsection{Visit Notes - Entity List}

Figure 9: user searches "infection," Visit Note - Entity List is filtered. "Infection" is added to search history

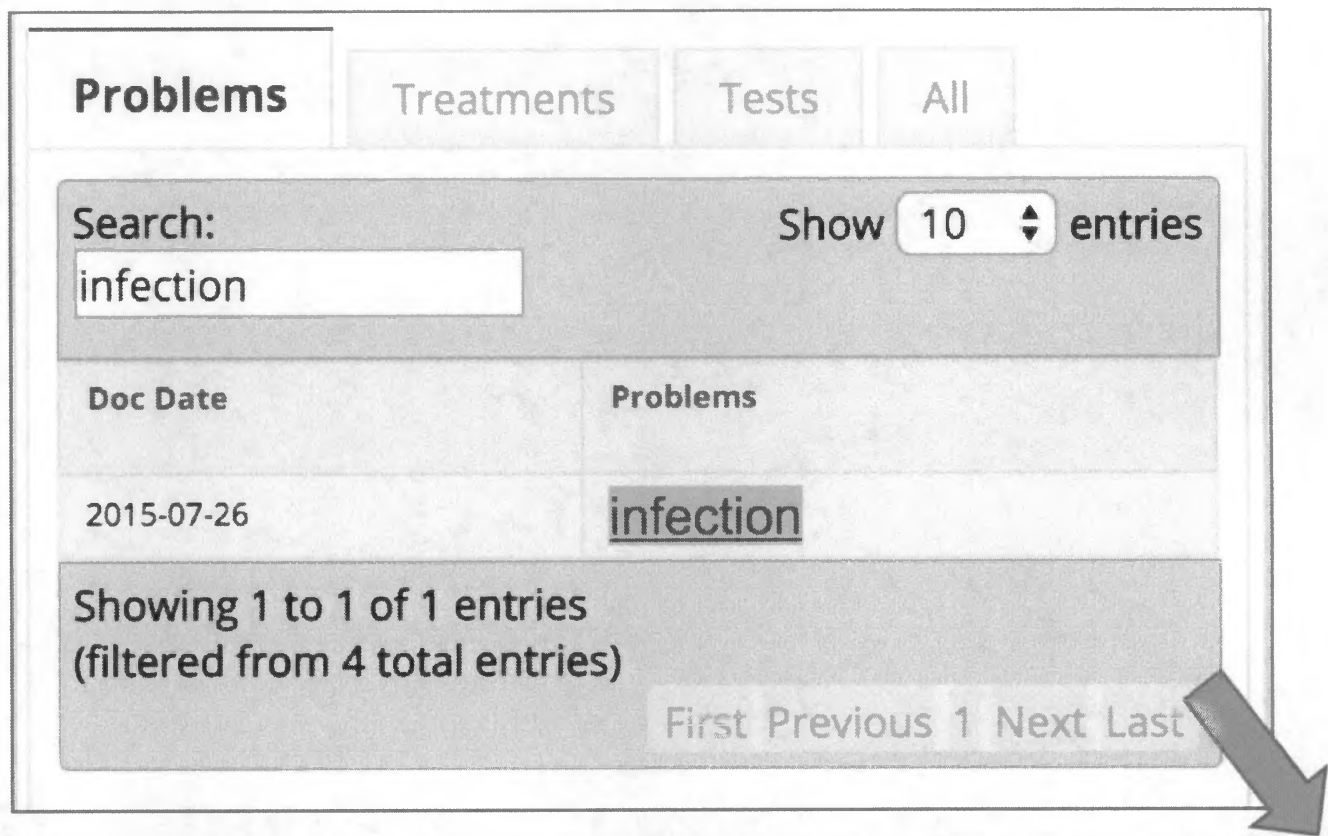

Search History: > hepatitis C cirrhosis > encephalopathy > end stage liver disease > encephalopathy > ascites > hematocrit > infection

The Visit Notes List provides several ways to filter and interact with the Visit Notes and Entities found in them. The Visit Notes List has three main components, see figure 10.

1. Entity Type Filter tabs

2. Search Filter

3. Visit Note - Entity list 
Figure 10: Visit Note - Entity List Ul components

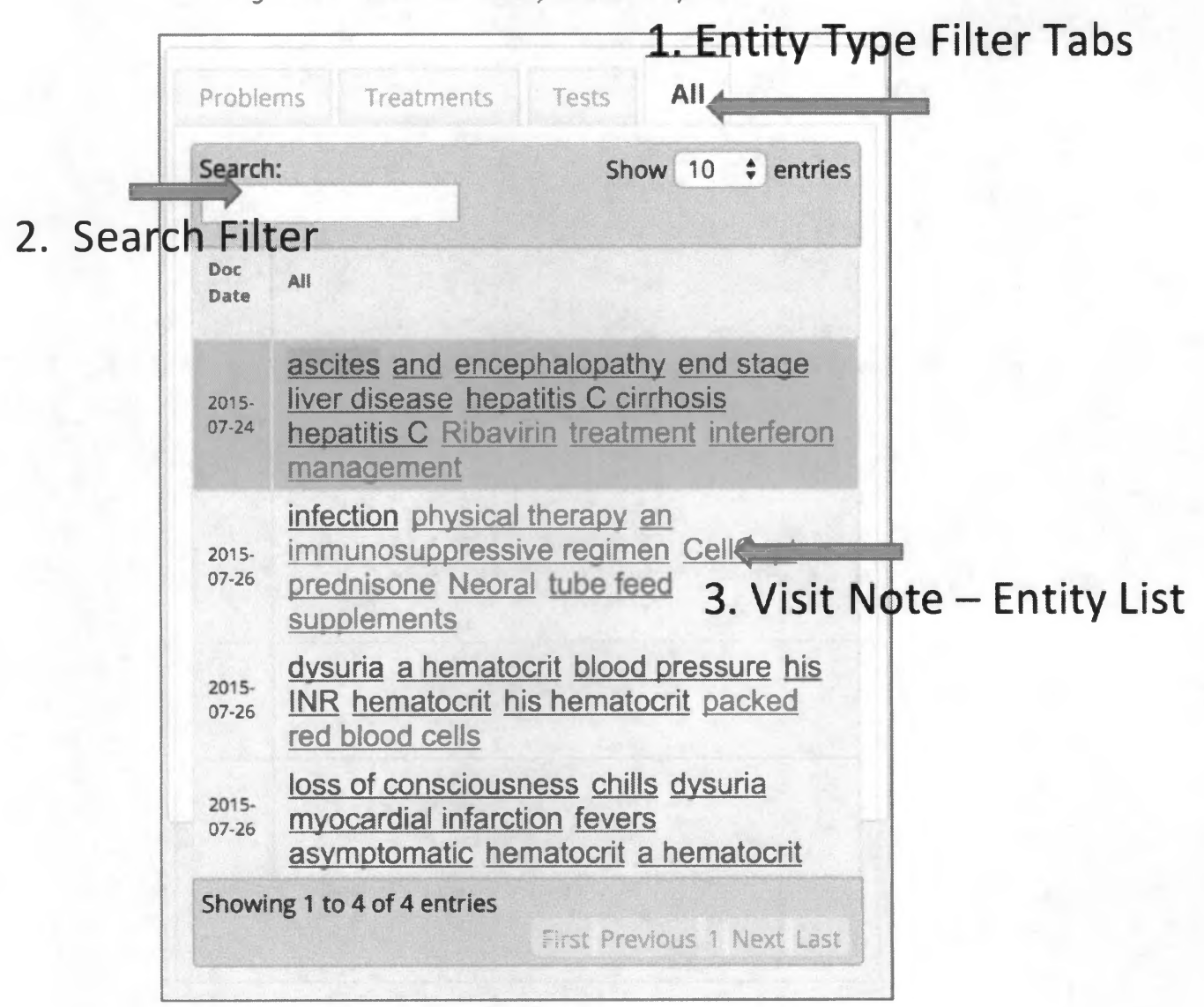

1. Entity Type Filter Tabs allow you to filter the Visit Note- Entity list for entities of one specific type by selecting its tab. Figure 11 shows the documents filtered for "Problem" entities. These filters also affect which entities are highlighted in the Visit Note Rendering. 
Figure 11: Visit Note - Entity List filtered for "Problems"

\begin{tabular}{|c|c|c|}
\hline Problems & Treatments & Tests \\
\hline \multicolumn{2}{|c|}{ Search: } & \multirow{2}{*}{ Show 10 : entries } \\
\hline $\begin{array}{l}\text { Doc } \\
\text { Date }\end{array}$ & Problems & \\
\hline $\begin{array}{l}2015 . \\
07-24\end{array}$ & \multicolumn{2}{|c|}{$\begin{array}{l}\text { ascites and encephalopathy end stage } \\
\text { liver disease hepatitis C cirrhosis } \\
\text { hepatitis C }\end{array}$} \\
\hline $\begin{array}{l}2015- \\
07-26\end{array}$ & \multicolumn{2}{|l|}{ infection } \\
\hline $\begin{array}{l}2015 . \\
07-26\end{array}$ & \multicolumn{2}{|c|}{$\begin{array}{l}\text { fevers loss of consciousness myocardial } \\
\text { infarction chest pain dysuria chills } \\
\text { shortness of breath with exertion baseline } \\
\text { shortness of breath asymptomatic }\end{array}$} \\
\hline $\begin{array}{l}2015- \\
07-26\end{array}$ & \multicolumn{2}{|c|}{$\begin{array}{l}\text { baseline shortness of breath shortness of } \\
\text { breath with exertion chest pain loss of } \\
\text { consciousness chills dysuria myocardial } \\
\text { infarction fevers asvmptomatic }\end{array}$} \\
\hline \multicolumn{3}{|c|}{ Showing 1 to 4 of 4 entries } \\
\hline & & First Previous 1 Next Last \\
\hline
\end{tabular}

1. The Search Filter lets the user search the entity list for specific entities. Only documents with entities that match the search text are displayed. Search is performed incrementally which means that when a user types the first 3 letters of "infection" then "myocardial infarction" will also be matched. Figure 12 shows this. 
Figure 12: Visit Note - Entity List as user types "inf"

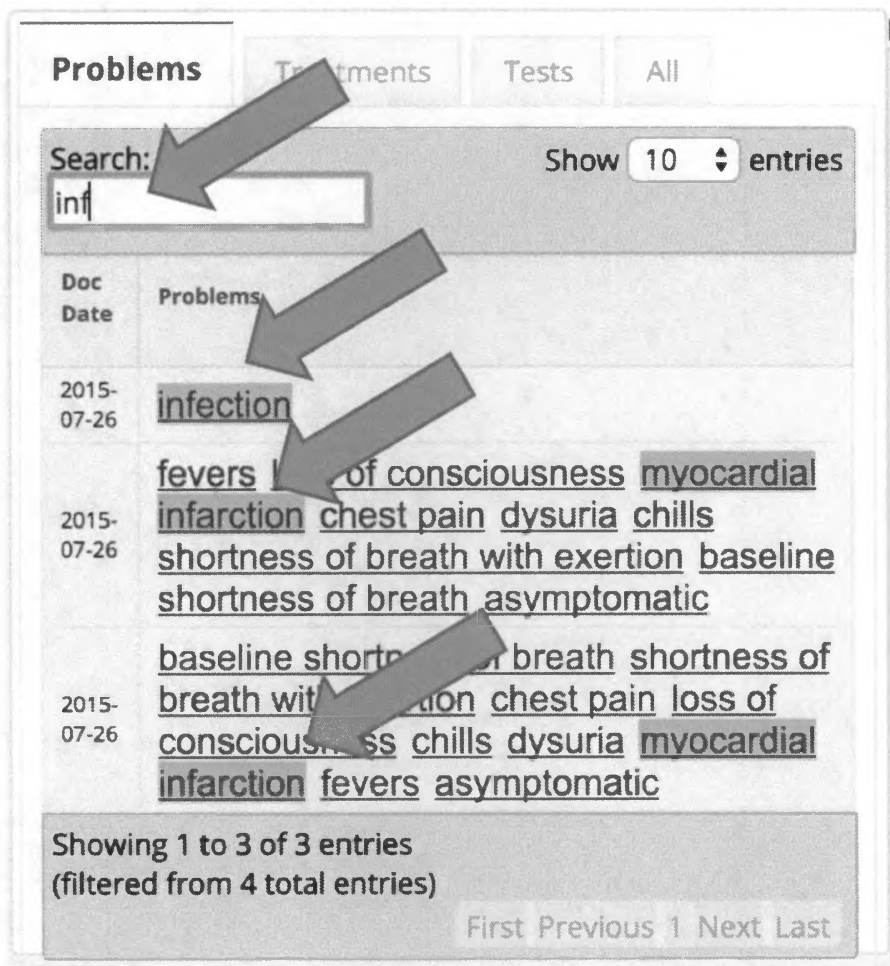

2. The Visit Note - Entity List is a table with 2 columns. The first column is the date of the Visit Note and the second is a list of Entities found in the note. When the user selects an entity from the Entity List, the corresponding visit note is rendered in the Visit Note Rendering. The selected entity is highlighted in the Visit Note and the text of the note is scrolled, if necessary, so that the selected entity is visible. Figure 13 shows the result when the user selects "encephalopathy" from the Visit Note List. 
Figure 13: Visit Note - Entity List and Document Rendering after "encephalopathy" is clicked.

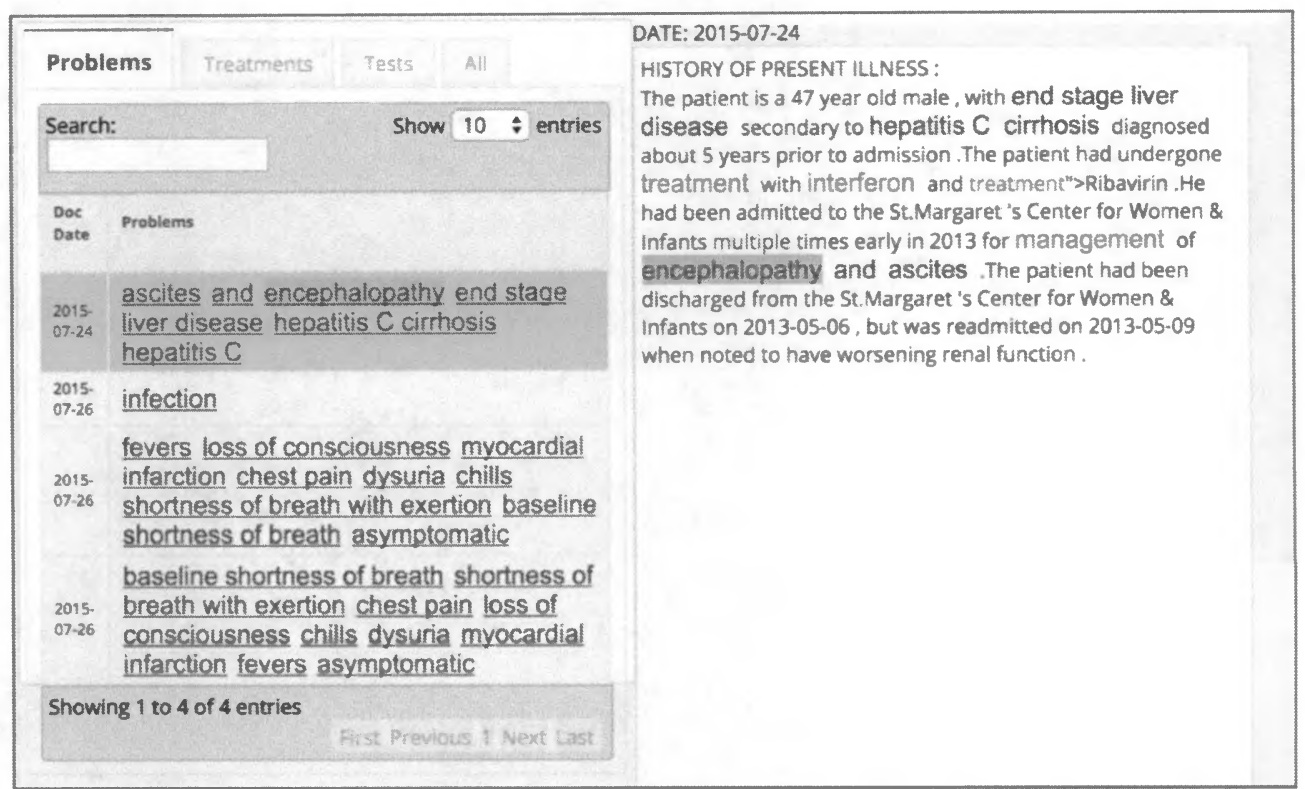

\subsection{Visit Note Rendering}

The Visit Note Rendering shows the text of a Visit Note with the identified entities color coded by entity type and enlarged. Also, the entity of interest is highlighted. Figure 14 shows the document rendering after the user selects the entity "ascites" from the Visit Note - Entity List. The document is rendered and "ascites" is highlighted. 


DATE: 2015-07-24
HISTORY OF PRESENT ILLNESS :
The patient is a 47 year old male, with end stage liver
disease secondary to hepatitis C cirrhosis diagnosed
about 5 years prior to admission .The patient had undergone
treatment with interferon and Ribavirin .He had been
admitted to the St.Margaret 's Center for Women \& Infants
multiple times early in 2013 for management of
encephalopathy and ascites .The patient had been
discharged from the St.Margaret 's Center for Women \&
Infants on 2013-05-06, but was readmitted on 2013-05-09
when noted to have worsening renal function.

\subsection{Admin Notification}

he admin notification button can be used to alert the admin of incorrectly tagged documents. If the user notices an error that the module has made, clicking on the link will prepare an e-mail with information about the currently visible Visit Note. The user will include a brief description of the problem and send it to the admin for future tuning of the algorithm. 
Figure 15: Notify the admin

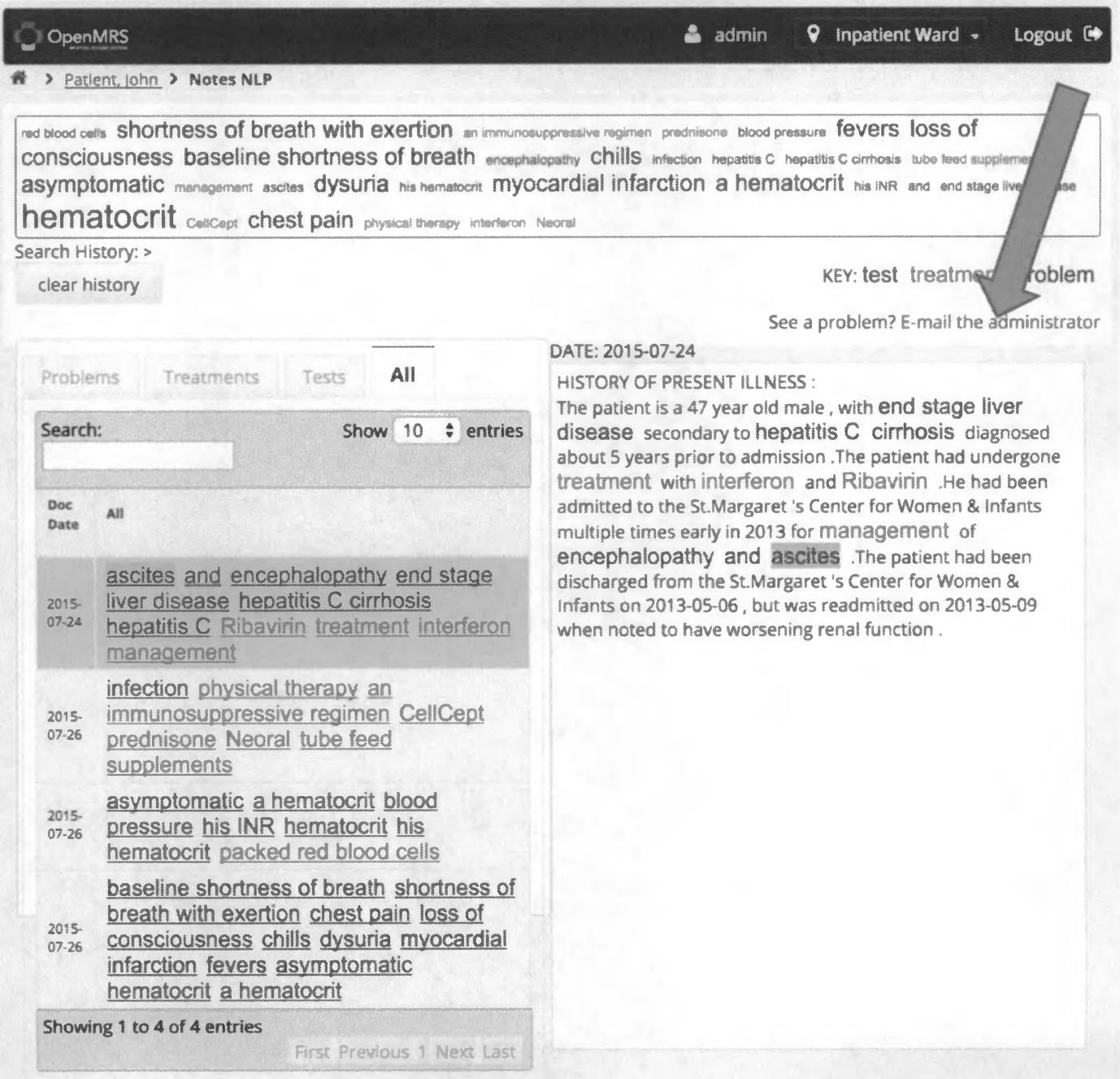


Appendix 2:

Visit Notes Analysis Module

Implementer's Guide 

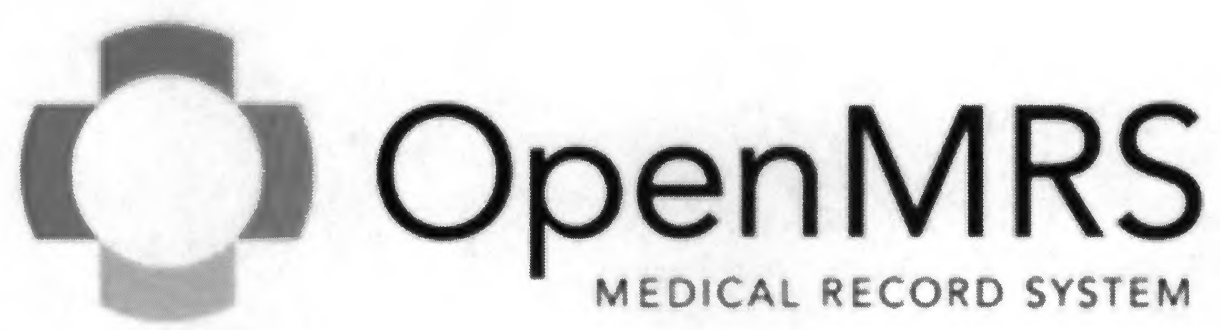

\section{Visit Notes Analysis Module Implementer's Guide}

Ryan Eshleman

Advisors: Dr. Barry Levine

Dr. Hui Yang 
Table of Contents:

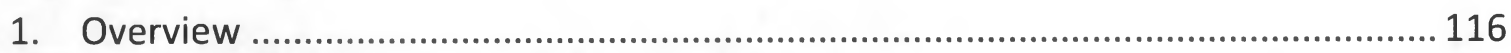

1.1. Visit Notes Analysis Module Overview.................................................... 116

1.2. Visit Note Annotator and Trainer Overview................................................ 117

1.2.1. Why Train a new model.............................................................. 118

1.2.2. Example Use Case................................................................... 118

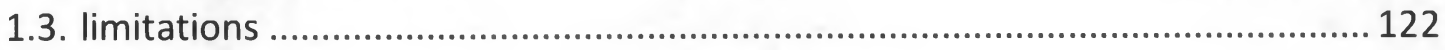

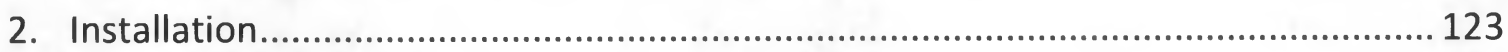

2.1. Module Installation ............................................................................ 123

2.2. Visit Note Annotator and Trainer installation ............................................ 124

3. Module Configuration and Maintenance ...................................................... 128

3.1. Defining Concept Class - Entity Type Mappings ...................................... 128

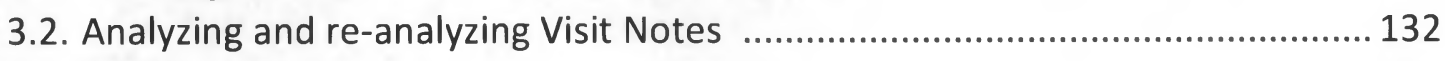

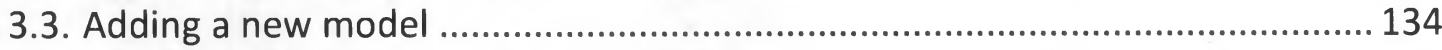

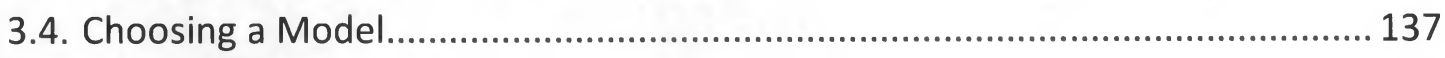

3.5. Running Reports ................................................................................. 139

4. Visit Note Annotator and Trainer User Interface ......................................... 143

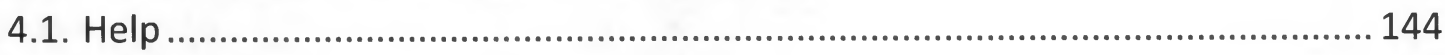

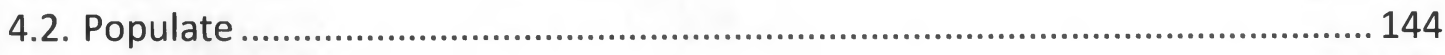

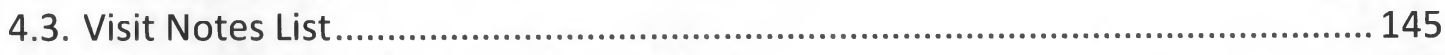

4.4. Visit Note Annotation Editor ............................................................... 145

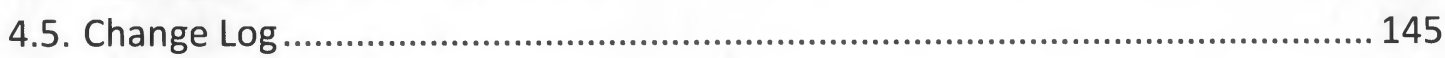

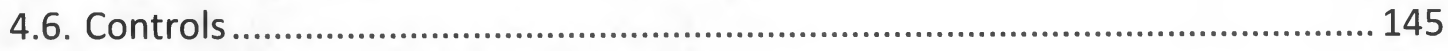

5. Visit Note Annotator and Trainer Usage .................................................... 145

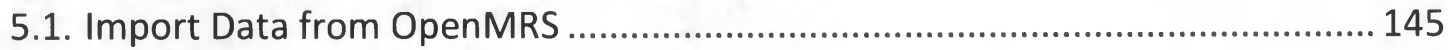

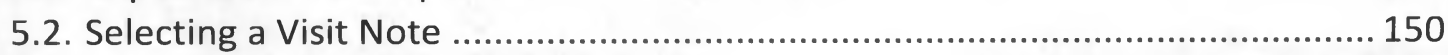

5.3. Editing Visit Note Annotations ............................................................ 150

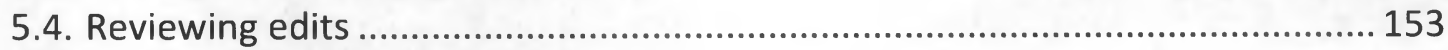

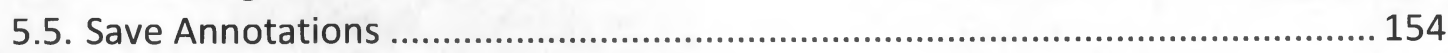

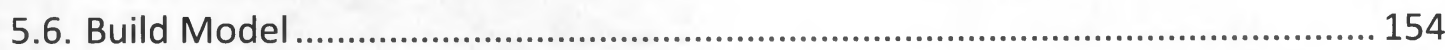

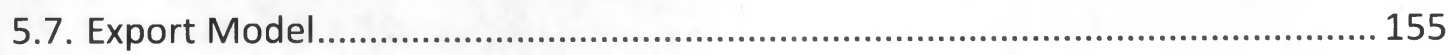




\section{Overview}

This document provides implementation details for the Visit Notes Analysis Module as well as the companion web application, the Visit Note Annotator and Trainer. These two pieces work together to allow the optimal performance of the system. Figure 1 shows the circular workflow between the two applications. The Visit Note Annotator and Trainer is used to improve the performance of the Visit Notes Analysis module. However, it is worth noting that while these applications can improve the performance of the system, it is not $100 \%$ accurate. More details on this are provided in section 1.3.

Figure 2: Visit Notes Analysis and Visit Notes Annotator and Trainer application workflow.

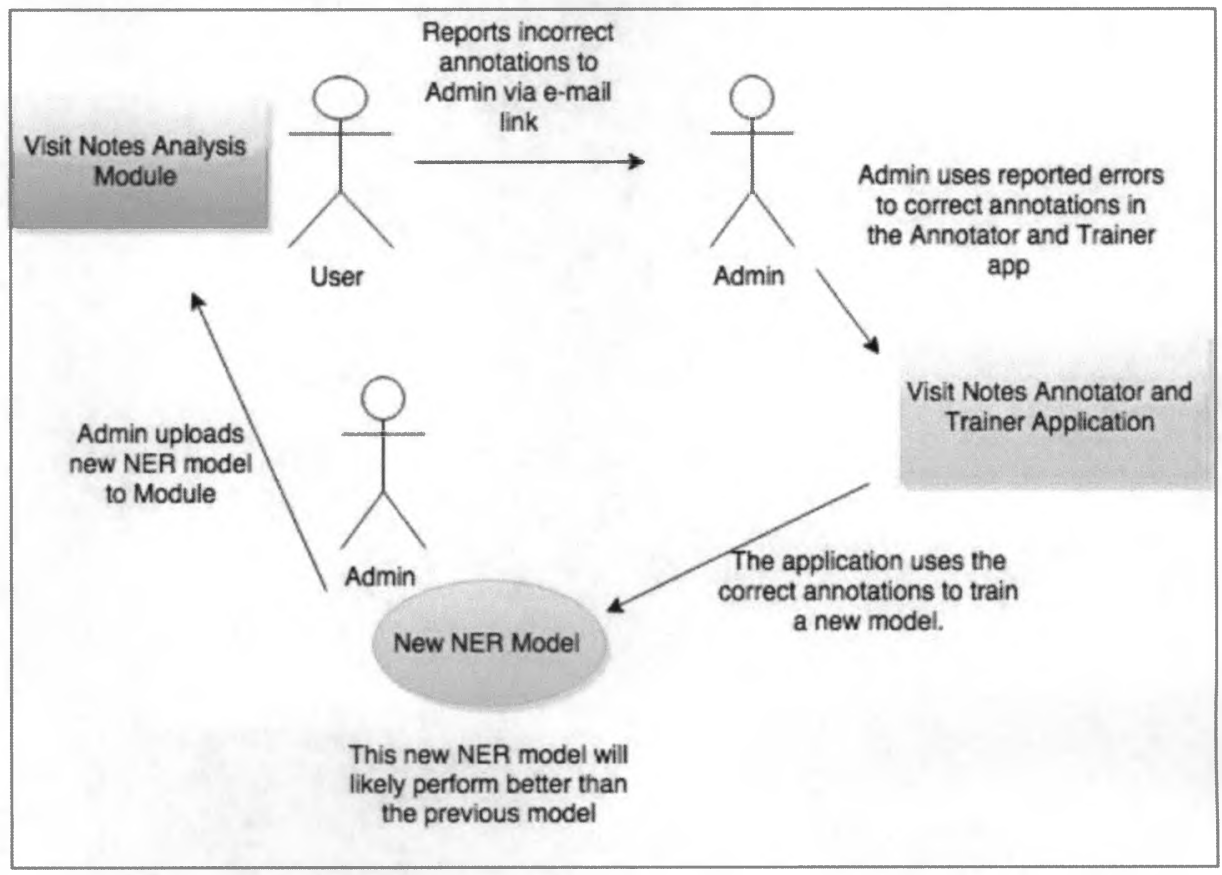

\subsection{Visit notes Analysis Module Overview}

The Visit Notes Analysis Module performs Named Entity Recognition (NER) on Visit Note text to identify where in the text a problem, treatment or test is mentioned. The underlying algorithm for identifying these entities has many moving parts, and this document will serve as a guide to configuring the NER functions for the best performance. 


\subsection{Visit Note Annotator and Trainer Overview}

The Name Entity Recognition algorithm used to identify medical concepts in the text of Visit Notes relies in part on a machine learning algorithm called Conditional Random Fields, implemented in BANNER (http://banner.sourceforge.net/). Like many machine learning algorithms, in order to show strong performance, it must first be trained on sample data. In our case this sample data consists of Visit Notes where entities of the types "Problem," "Treatment," and "Test" have been annotated. The text area in Figure 2 shows an example of annotated data. The process of providing correctly annotated data

Figure 2: The Visit Note Annotator and Trainer application

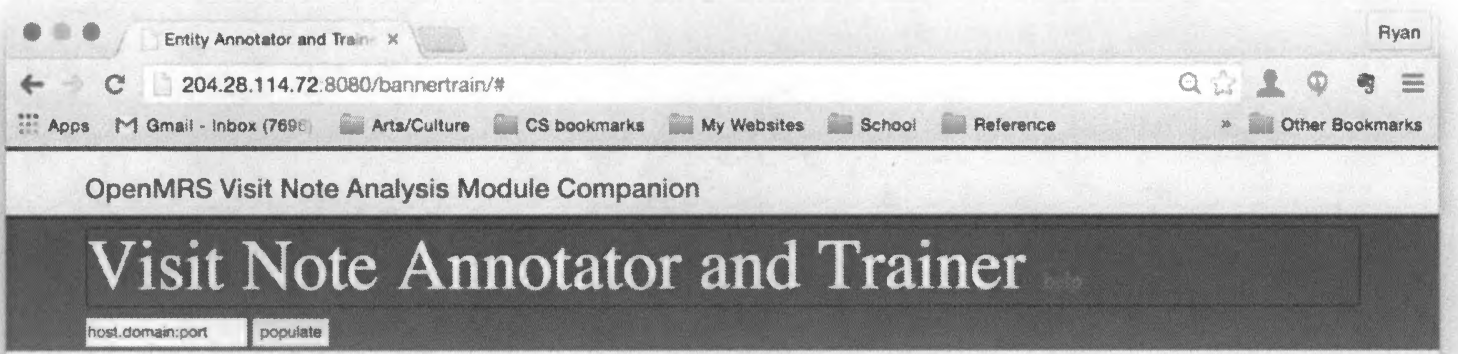

\begin{tabular}{|c|c|c|}
\hline \multicolumn{3}{|c|}{ Show 10 : entries } \\
\hline \multicolumn{3}{|c|}{ Search: } \\
\hline Docto & Date & $\begin{array}{l}\text { Name } \\
\text { MRN }\end{array}$ \\
\hline 1 & $\begin{array}{l}07 / 24 / 2015 \\
15: 08: 15\end{array}$ & $\begin{array}{l}\text { Patient, John } \\
\text { 100-8 }\end{array}$ \\
\hline 2 & $\begin{array}{l}07 / 26 / 2015 \\
12: 03: 42\end{array}$ & $\begin{array}{l}\text { Patient, John } \\
100-8\end{array}$ \\
\hline 3 & $\begin{array}{l}07 / 28 / 2015 \\
18: 33: 00\end{array}$ & $\begin{array}{l}\text { Patient, John } \\
\text { 100-8 }\end{array}$ \\
\hline 4 & $\begin{array}{l}\text { 07/28/2015 } \\
\text { 18:35:46 }\end{array}$ & $\begin{array}{l}\text { Patiert, John } \\
100-8\end{array}$ \\
\hline Dock & Date & $\begin{array}{l}\text { Name } \\
\text { MRN }\end{array}$ \\
\hline \multicolumn{3}{|c|}{ Showing 1 to 4 of 4 entres } \\
\hline & Prowous & 1 Next \\
\hline
\end{tabular}

\begin{tabular}{|c|c|c|c|c|}
\hline \multirow{5}{*}{ 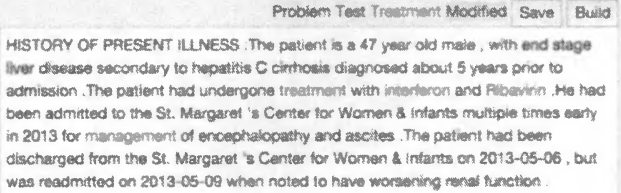 } & \multicolumn{4}{|c|}{ Change Log } \\
\hline & \multicolumn{4}{|c|}{ Show 10 : entries } \\
\hline & \multicolumn{4}{|l|}{ Search: } \\
\hline & & \multirow{2}{*}{\multicolumn{2}{|c|}{$\begin{array}{l}\text { worsening } \\
\text { renail Anction }\end{array}$}} & \\
\hline & $\begin{array}{l}\text { worsenting } \\
\text { renal function }\end{array}$ & & & undo \\
\hline & From & \multicolumn{2}{|l|}{ To } & \\
\hline & \multicolumn{3}{|c|}{ Showing 1 to 1 of 1 entries } & \\
\hline & & \multicolumn{2}{|l|}{ Prewious } & Nent \\
\hline
\end{tabular}


to the algorithm for it to learn from is called training a model. The algorithm builds a model of what entities of the different types look like, and uses this model to identify entities in new text.

\subsubsection{Why Train a new model?}

The default model shipped with the Visit Notes Analysis module is trained on a public set of annotated clinical notes provided by 12B2 (i2b2.org). This model showed strong performance when used to tag documents in the 12B2 corpus, but textual and linguistic differences between 12B2 documents and Visit Notes encountered in the field might degrade the performance of the default model and cause it to overlook entities or misidentify them.

We have provided the Visit Note Annotator and Training application to allow the OpenMRS administrators to use Visit Notes captured in their database to train a new model in the hopes that this model will improve performance of the system. Henceforth, when discussing the Trainer, the user will refer to the administrator responsible for training.

\subsubsection{Example Use Case}

To illustrate how the OpenMRS module and Language Model Trainer application work together, consider the following use case. A user is examining the visit notes for patient John D. Patient. Figure 3 shows the annotations made to the Visit Note. An annotation is indicated by different text colors. Red for problem, green for treatment and blue for test. Here, "prednisone" is green, indicating it is a treatment. The user notices that one or more words in the visit note are not tagged correctly. "Prednisone" is correctly identified as a treatment, but "Neoral", and "CellCept" should have been identified as treatments, but were not, neither was "physical therapy". "liver function tests" should have been recognized as a test, but it was not. 
Figure 3: Annotations by a model that performs poorly on this text. Many entities are not recognized.

\section{DATE: 2015-07-26}

He was advanced to a regular house diet later on postop day 3 .The patient was advanced per protocol to an immunosuppressive regimen of prednisone Neoral, and CellCept. The patient 's mental status remained essentially clear throughout the entire postoperative period. The patient started ambulating with the assistance of physical therapy following transfer to the surgical floor. At the time of discharge , the patient was independent, ambulating, and functioning well .The patient 's appetite improved significantly, and at the time of discharge the patient was on a regular diet with no tube feed supplements deemed necessary .The patient 's liver function tests all improved appropriately by the time of discharge. The patient 's surgical incision was also healing well by the time of discharge with no evidence of infection.

A user (e.g. clinician using OpenMRS) can send an e-mail to the admin to report the incorrect annotations. An example tamplate e-mail is shown in figure 4. It provides the admin with the document number, the date and time the note was recorded, the patient Medical Record Number and the text of the Visit Note. This info will allow the admin to find and make corrections in the next steps. 
Figure 4: Template email to admin to report errors.

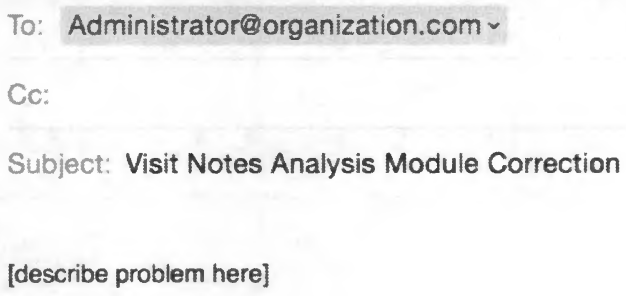

The patient is a 47 year old male, with end stage liver disease secondary to hepatitis $C$ cirrhosis diagnosed about 5 years prior to admission. The patient had undergone treatment with Ribavirin. He had been admitted to the St.Margaret 's Center for Women \& Infants multiple times early in 2013 for management of encephalopathy and ascites. The patient had been discharged from the St.Margaret 's Center for Women \& Infants on 2013-05-06, but was readmitted on 2013-05-09 when noted to have worsening renal function I

The Admin can then navigate to the Visit Note Annotator and Trainer application, load the annotated visit notes, and correct the errors that were observed in the OpenMRS module. In figure 5, the documents are loaded and we can see that the mistakes match those found in the OpenMRS module. Figure 6 shows the corrections to the annotations made by the admin. These corrections are reflected both on the document rendering as well as the Change Log. 


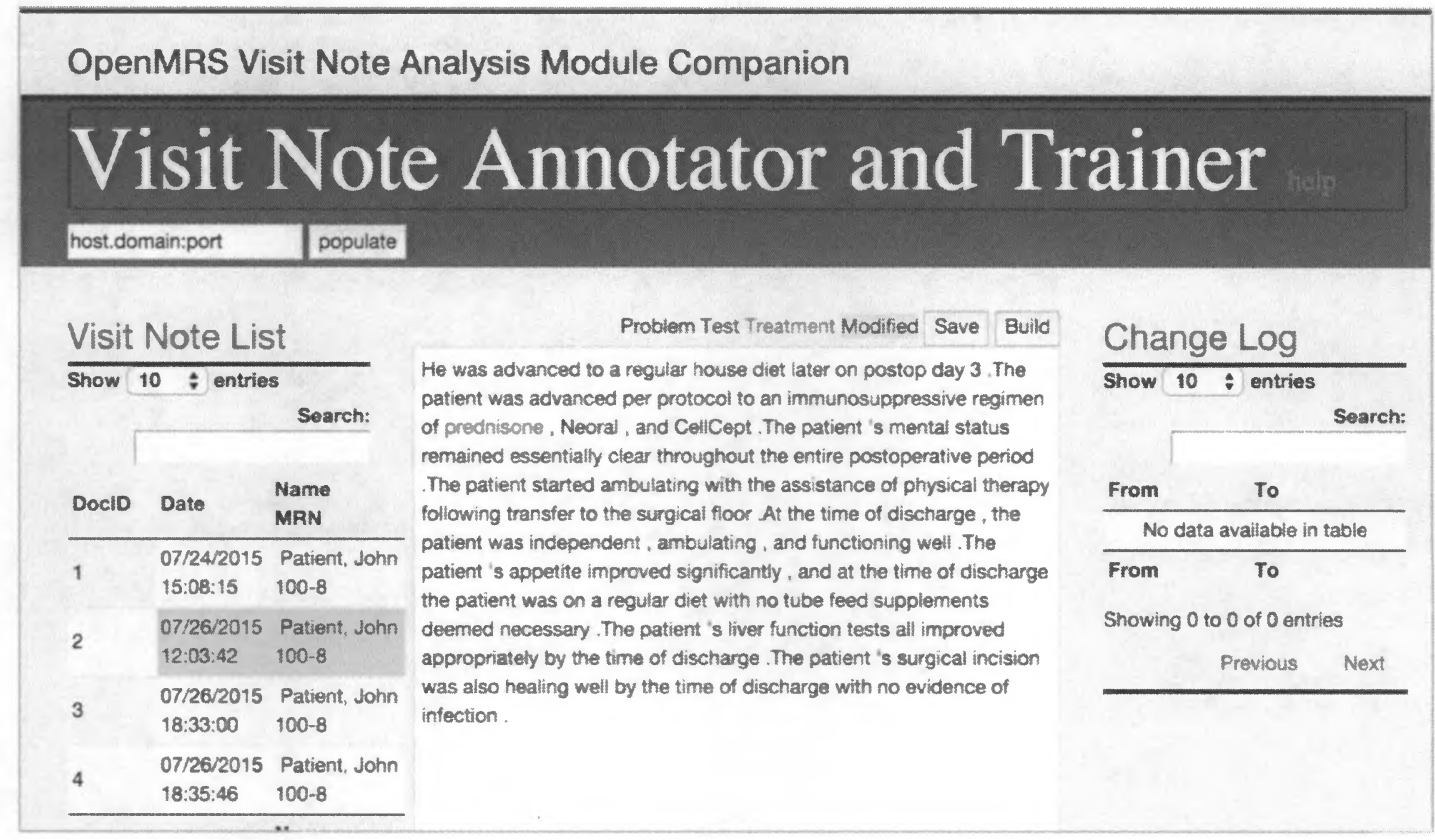

Figure 6: Admin corrects the annotations

\begin{tabular}{|c|c|c|c|c|}
\hline \multirow{2}{*}{\multicolumn{2}{|c|}{$\begin{array}{l}\text { Problem Test Treatment Modified Save } \text { Build } \\
\text { He was advanced to a regular house diet later on postop day } 3 \text {.The } \\
\text { patient was advanced per protocol to an immunosuppressive regimen } \\
\text { of prednisone, Neoral, and CellCept.The patient 's mental status } \\
\text { remained essentially clear throughout the entire postoperative period }\end{array}$}} & \multicolumn{3}{|c|}{ Change Log } \\
\hline & & Show 10 & entries & Search: \\
\hline \multirow{5}{*}{\multicolumn{2}{|c|}{$\begin{array}{l}\text { The patient started ambulating with the assistance of physical therapy } \\
\text { following transfer to the surgical floor.At the time of discharge, the } \\
\text { patient was independent, ambulating, and functioning well. The } \\
\text { patient 's appetite improved significantly, and at the time of discharge } \\
\text { the patient was on a regular diet with no tube feed supplements } \\
\text { deemed necessary. The patient 's liver function tests all improved } \\
\text { appropriately by the time of discharge. The patient 's surgical incision } \\
\text { was also healing well by the time of discharge with no evidence of } \\
\text { infection. }\end{array}$}} & Neoral & Neoral & undo \\
\hline & & CellCept & CellCept & undo \\
\hline & & $\begin{array}{l}\text { physical } \\
\text { therapy }\end{array}$ & $\begin{array}{l}\text { physical } \\
\text { therapy }\end{array}$ & undo \\
\hline & & $\begin{array}{l}\text { liver function } \\
\text { tests }\end{array}$ & $\begin{array}{l}\text { liver } \\
\text { function } \\
\text { tests }\end{array}$ & undo \\
\hline & & From & To & \\
\hline
\end{tabular}

When the admin is satisfied with the document annotations, the admin selects the "Build" button and the text and annotations are used to train a new BANNER Language Model. The admin is able to download this model from the training application and upload it to the OpenMRS implementation via a configurations page. 
This new model is used to re-analyze the text and it is able to successfully find the correct entities in the text. Figure 7 shows the results of the new analysis.

In this way, the OpenMRS can improve their language model as they encounter errors.

Figure 7: The new model re-analyzed the text and correctly identified the entities.

DATE: $2015-07-26$

He was advanced to a regular house diet later on postop day 3 .The patient was advanced per protocol to an

immunosuppressive regimen of prednisone Neoral , and CellCept .The patient 's mental status rem red essentially clear $t$ aghout the entire postoperative Aariod .The patient started bulating with the assistance of physical therapy

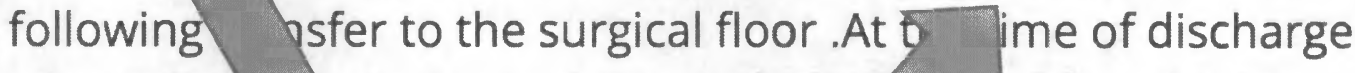
, the patien was independent, ambulat and functioning well. The patient 's appetite improved ificantly, and at the time of discharge the patipnt was on ay egular diet with no tube feed supp/ cremed necessary. The patied 's liver function tests all improved appropriately of discharge. The patient 's surgical incision was also healing well by the time of discharge with no evidence of infection .

\subsection{Limitations}

During the development of this module, we performed evaluations of several NER algorithms in order to choose the best one to use here. During these evaluations it became clear that the current state of NER is not perfect. This module uses the BANNER NER system and our measurements showed that of the entities it finds, about $80 \%$ are correct. Also, it tends to find about $70 \%$ of the entities in the text. While this performance is good, it indicates that the results of this module should not be relied upon for perfect accuracy, and should be used as a tool to assist in analyzing Visit Notes. 


\section{Installation}

\subsection{Visit Notes Analysis Module Installation \\ Requirements:}

Jdk 1.7

Requires OpenMRS versions 2.x, core 1.11.x

[Support for $1.9 x$ is not available yet.]

\section{Installation}

Installation of the module follows the standard OpenMRS module installation procedures.

NOTE: installing this module requires you to restart your OpenMRS installation

1. Navigate to OpenMRS Administration Page

2. Select the "Manage Modules" Link

3. Upload the .omod file through the "add or upgrade module" button. Note: the .omod file is rather large $\sim 48 \mathrm{MB}$ so the upload may take some time

4. Restart your OpenMRS installation. 
Figure 8: Upload the module via OpenMRS's Manage Modules page

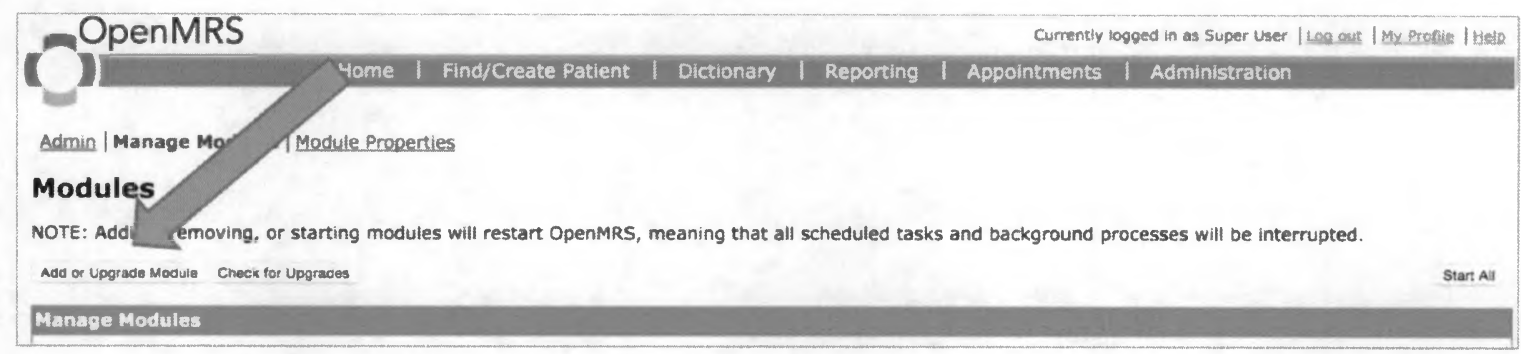

\subsection{Visit Notes Annotator and Trainer installation}

This application has been tested in the following environment:

Java Servlet Container: Apache Tomcat 6 servlet container

JVM: Java 1.7

OS:

Ubuntu Linux 14.04

For current Tomcat installation instructions, please refer to one of the many Tomcat installation guides available on the web. For example:

https://help.ubuntu.com/Its/serverguide/tomcat.html

Note: be sure to install the Tomcat administration pages webapps. 


\section{Installing the application via Tomcat Application Manager}

1. Navigate and login to the Tomcat Application Manager, this is often at [serverURL]:[port]/manager/html.

Figure 9: Tomcat Application Manager page

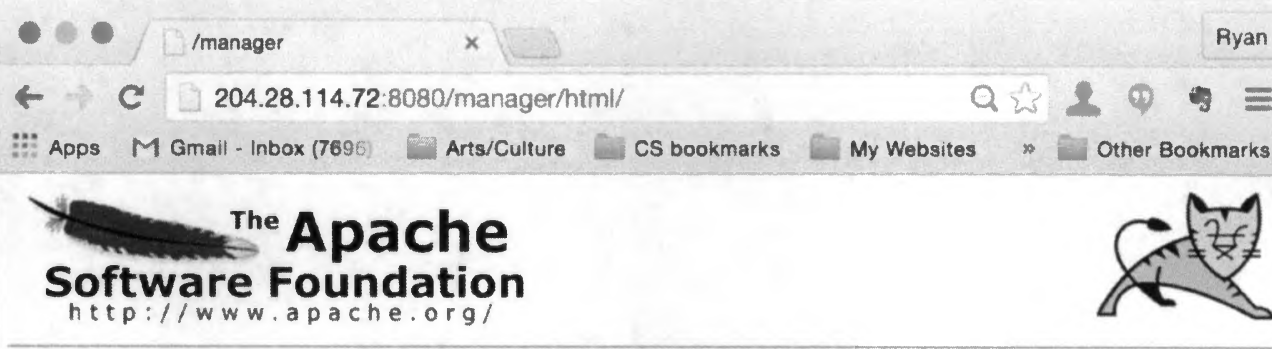

\section{Tomcat Web Application Manager}

\begin{tabular}{|c|c|c|c|}
\hline Message: & & & \\
\hline \multicolumn{4}{|l|}{ Manager } \\
\hline List Applications & HTML Manager Help & Manager Help & Server Status \\
\hline
\end{tabular}

\begin{tabular}{|c|c|c|c|c|}
\hline \multicolumn{5}{|c|}{ Applications } \\
\hline Path & Display Name & Running & Sessions & Commands \\
\hline \multirow[b]{2}{*}{ ! } & & \multirow[b]{2}{*}{ true } & \multirow[b]{2}{*}{$\underline{0}$} & Start Stop Reload Undeploy \\
\hline & & & & Exore sevsions with idle $\geq 30$ minutes \\
\hline \multirow[b]{2}{*}{ Imanacer } & \multirow[b]{2}{*}{ Tomcat Manager Application } & \multirow[b]{2}{*}{ true } & \multirow[b]{2}{*}{1} & Start Stop Reload Undeploy \\
\hline & & & & Expire sessions with idie $\geq 30$ \\
\hline \multirow[b]{2}{*}{ lopenmrs } & \multirow[b]{2}{*}{ OpenMAS } & \multirow[b]{2}{*}{ true } & \multirow[b]{2}{*}{1} & Stan Stop Reload Undeplor \\
\hline & & & & Exare sessions with idle $\geq 30$ \\
\hline
\end{tabular}

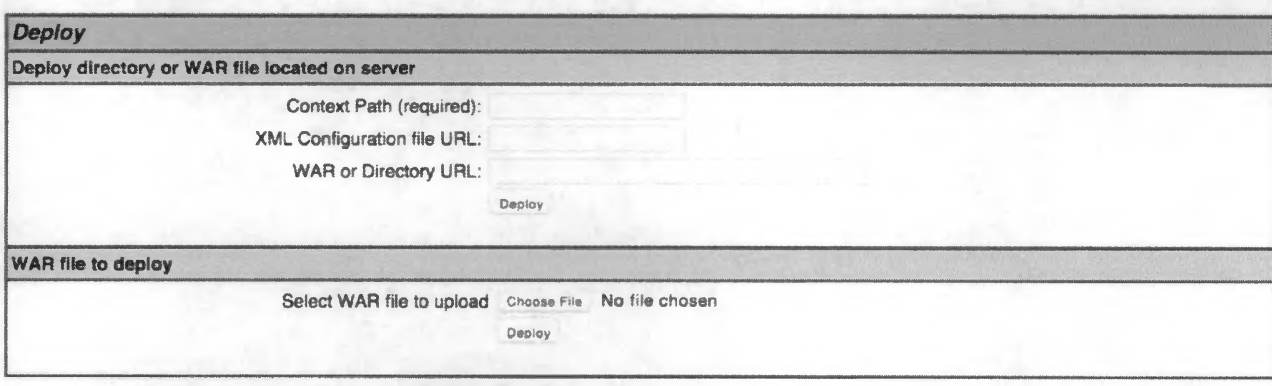


Fiqure 10: upload the war file

\begin{tabular}{|c|c|c|}
\hline Deploy & & \\
\hline Deploy directory or & le located on server & \\
\hline & Context Path (required): & \\
\hline & XML Configuration file URL: & \\
\hline & WAR or Directory URL: & \\
\hline & & Depioy \\
\hline WAR file to deploy & & \\
\hline & Select WAR file to upload & Choose File bannertrain war \\
\hline
\end{tabular}

2. upload the bannertrain.war file via the "WAR file to deploy" pane

3. Click "Deploy"

4. After the files finishes uploading to the server (may take several minutes, depending on connection). The application will be visible in the "Applications" pane, figure 11.

Fiaure 11: The abolication is runnina and accessible at the indicated path

\begin{tabular}{|c|c|c|c|c|}
\hline \multicolumn{5}{|c|}{ Applications } \\
\hline Path & Display Name & Running & Sessions & Commands \\
\hline \multirow[b]{2}{*}{$l$} & & \multirow[b]{2}{*}{ true } & \multirow[b]{2}{*}{$\underline{0}$} & Start Stop Reload Undeploy \\
\hline & & & & Expire sessions with idle $\geq 30$ \\
\hline \multirow[b]{2}{*}{ Bbannertrain } & \multirow[b]{2}{*}{ bannertrain } & \multirow[b]{2}{*}{ true } & \multirow[b]{2}{*}{$\underline{0}$} & Start Stop Reload Undeploy \\
\hline & & & & Expire sessions with idle $\geq 30$ \\
\hline \multirow[b]{2}{*}{ Imanager } & \multirow{2}{*}{ Tomcat Manager Application } & \multirow[b]{2}{*}{ true } & \multirow[b]{2}{*}{1} & Start Stop Reload Undeploy \\
\hline & & & & Expire sessions with idle $\geq 30 \quad$ minutes \\
\hline \multirow[b]{2}{*}{ lopenmrs } & \multirow[b]{2}{*}{ OpenMRS } & \multirow[b]{2}{*}{ true } & \multirow[b]{2}{*}{1} & Start Stop Reload Undeploy \\
\hline & & & & Expire sessions with idle $\geq 30$ \\
\hline
\end{tabular}

5. Clicking through the link will bring you to the deployed web app, figure 12 . 
Fiqure 12: Visit Note Annotator and Trainer application with no Visit Notes loaded

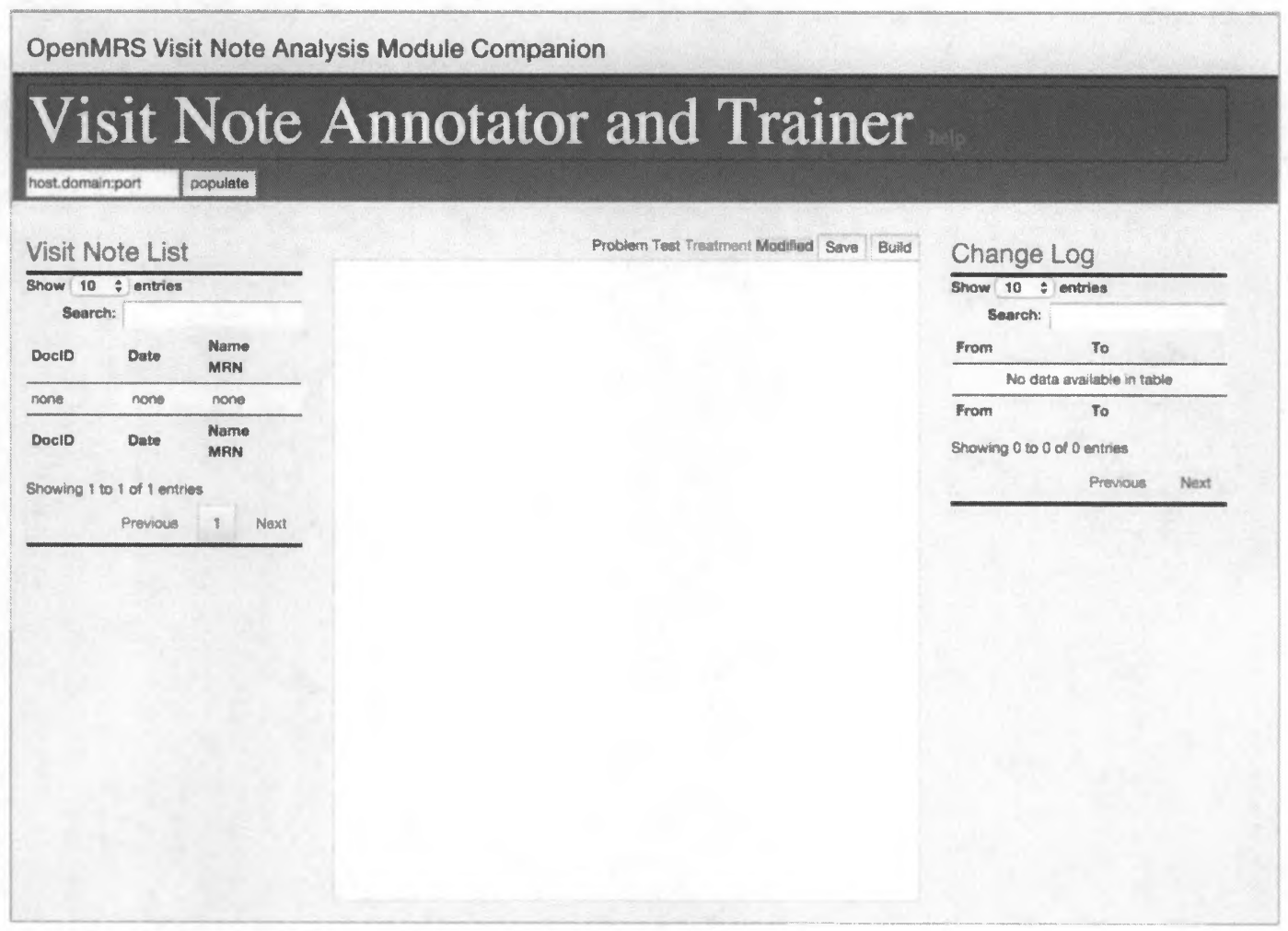




\section{Module Configurations and Maintenance}

\subsection{Defining Concept Class - Entity Mappings}

The first step of the Named Entity Recognition algorithm uses the Concept Dictionary to perform string matching against concepts in the dictionary. Because our module tags entities into three distinct entity types but an OpenMRS implementation often has many more concept classes, we allow the user to provide a many-to-one mapping from OpenMRS concept classes to entity type.

To set this mapping:

1. Navigate to the "Manage Module" page from the OpenMRS Administration page 
Fiqure 13: OpenMRS Administration paqe

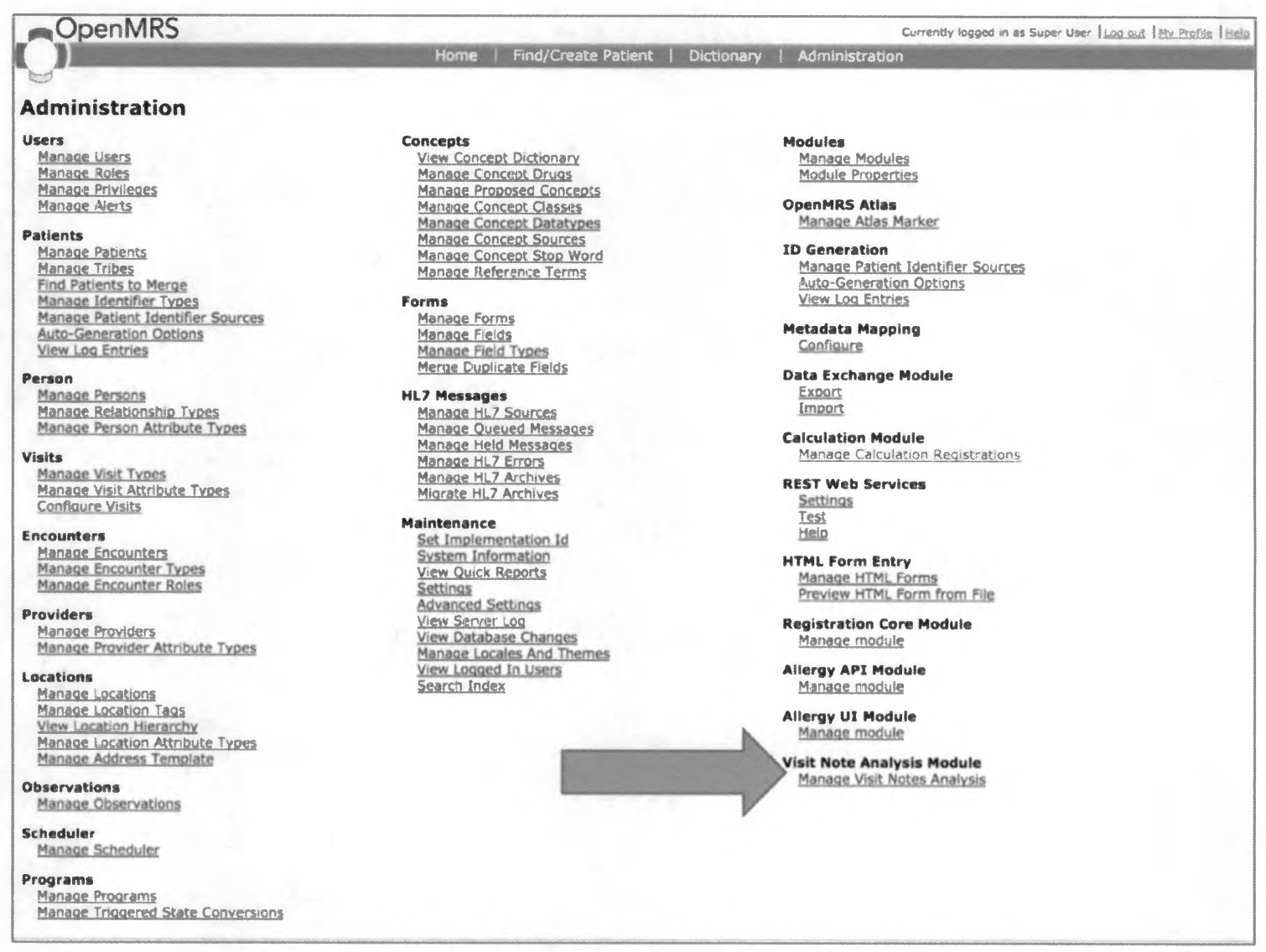

2. Locate the input fields labeled "Problems", "Treatments", and "Tests." The values should be a comma separated list of the Concept Classes that match to each Entity class. A list of concept classes can be found at the "Manage Concept Classes" link from the Administration page. The "Manage Tagger" sections maintains configurations related to the NER tagger. 


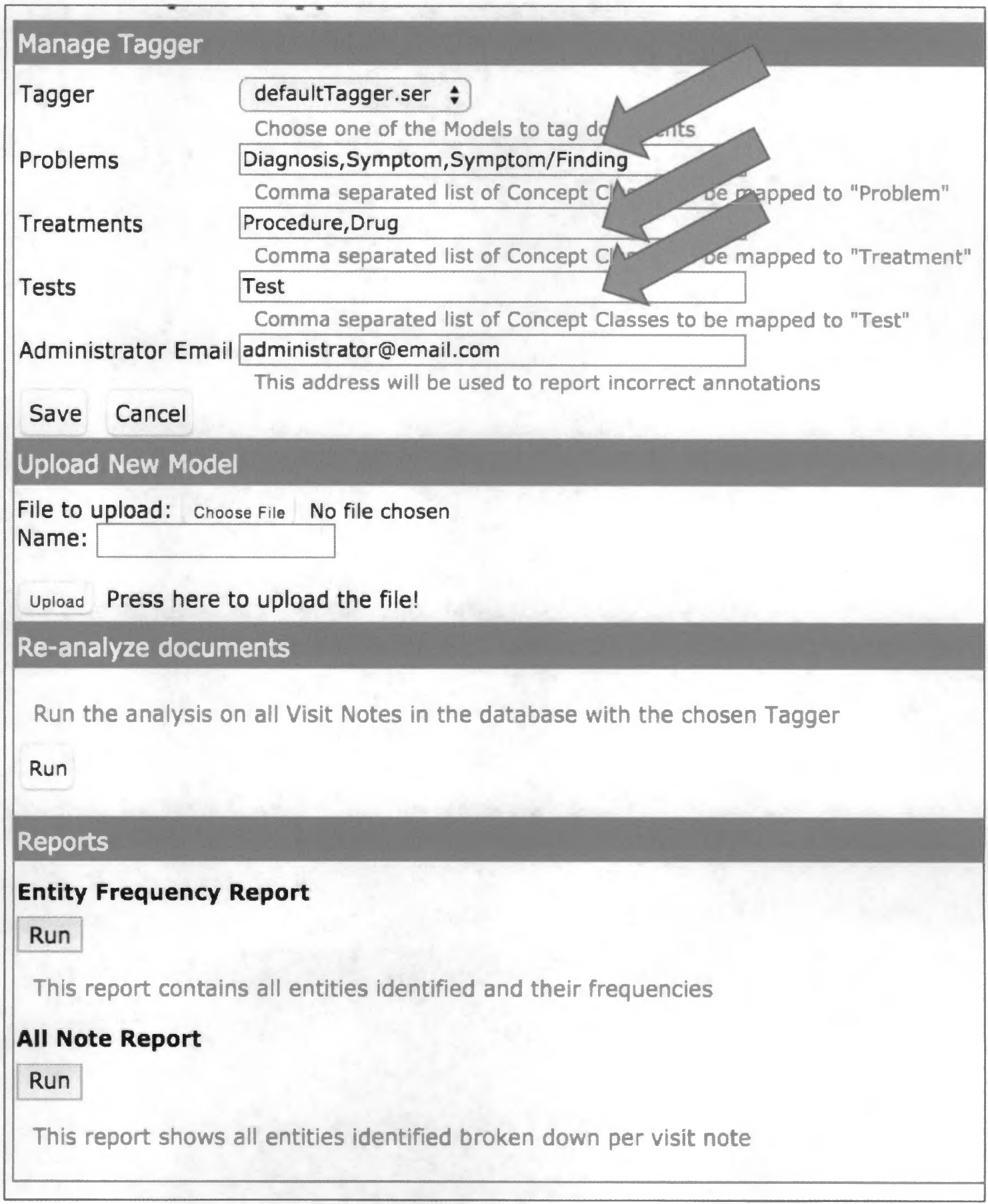


Figure 15 shows how to navigate to the Concept Class Manager from the Administration page and figure 16 shows the list of Concept Classes:

\begin{tabular}{|c|c|c|c|}
\hline \multicolumn{4}{|c|}{ Fiaure 15: Administration paqe - findina Concept Classes page } \\
\hline & Home I Find/Create Patient & Dictionary & Administration \\
\hline \multicolumn{4}{|l|}{ Administration } \\
\hline Users & Concepts & & Modules \\
\hline Manage Users & View Concept Dictionary & & Manage Modules \\
\hline Manage Roles & Manage Conceat Druas & & Module Properties \\
\hline Manage Privileges & Manage Proposed Concepts & & \\
\hline Manaqe Alerts & Manage Concept Classes & & OpenMRS Atlas \\
\hline Patients & $\frac{\text { Manage Concept Datatydes }}{\text { Manage Conceot Sources }}$ & & Manage Atlas Marker \\
\hline Manace Patients & $\begin{array}{l}\text { Manade Conceot Sources } \\
\text { Manage Conceot Stoo Word }\end{array}$ & & eneration \\
\hline Manage Tribes & Manage Reference Terms & & hage Patient Identifier Sources \\
\hline Find Patients to Merqe & Manoge acrefrence terims & & Auto-Generation Options \\
\hline Manaqe Identifier Types & Forms & & View Loq Entries \\
\hline Manage Patient Identifier Sources & Manage Forms & & \\
\hline $\begin{array}{l}\text { Auto-Generation Options } \\
\text { View Log Entries }\end{array}$ & Manage Fields & & $\begin{array}{l}\text { Metadata Mapping } \\
\text { Confiqure }\end{array}$ \\
\hline Person & Merge Duplicate Fields & & Data Exchange Module \\
\hline Manage Persons & HL7 Messages & & Export \\
\hline Manage Relationship Types & Manage HL7 Sources & & Import \\
\hline Manage Person Attribute Types & Manage Queued Messages & & Calculation Module \\
\hline Visits & $\begin{array}{l}\text { Manage Held Messages } \\
\text { Manage HL7 Errors }\end{array}$ & & Manage Calculation Registrations \\
\hline Manace Visit Types & Manage HL7 Archives & & \\
\hline $\begin{array}{l}\text { Manage Visit Attribute Trpes } \\
\text { Confiaure Visits }\end{array}$ & Migrate HL7 Archives & & Settinas \\
\hline
\end{tabular}

Figure 16: Concept Classes shown inside red box

\begin{tabular}{|c|c|}
\hline $\begin{array}{l}\text { Name } \\
\text { Test } \\
\text { Procedure } \\
\text { Druq } \\
\frac{\text { Diagnosis }}{\text { Finding }} \\
\underline{\text { Anatomy }} \\
\underline{\text { Question }} \\
\text { LabSet } \\
\underline{\text { MedSet }} \\
\underline{\text { ConvSet }} \\
\underline{\text { Misc }} \\
\frac{\text { Svmptom }}{\text { Svmptom/Finding }} \\
\underline{\text { Specimen }} \\
\frac{\text { Misc Order }}{\text { Frequency }} \\
\frac{\text { Pharmacologic Druq Class }}{\text { Units of Measure }} \\
\text { Delete Selected Concept Classes }\end{array}$ & $\begin{array}{l}\text { Description } \\
\text { Acq. during patient encounter (vitals, labs, etc.) } \\
\text { pescribes a clinical procedure } \\
\text { prug } \\
\text { fonclusion drawn through findings } \\
\text { Practitioner observation/finding } \\
\text { Anatomic sites / descriptors } \\
\text { puestion (eg, patient history, SF36 items) } \\
\text { erm to describe laboratory sets } \\
\text { ferm to describe medication sets } \\
\text { erm to describe convenience sets } \\
\text { ferms which don't fit other categories } \\
\text { Patient-reported observation } \\
\text { pbservation that can be reported from patient or found on exam } \\
\text { Body or fluid specimen } \\
\text { Prderable items which aren't tests or drugs } \\
\text { A concept used for capturing frequency information such as for medication ordering. } \\
\text { flass of medications based on pharmacologic properties as opposed to therapeutic properties } \\
\text { for prescribing and dispensing }\end{array}$ \\
\hline
\end{tabular}




\subsection{Analyzing and Re-analyzing Visit Notes}

When the module is first installed, there might be a corpus of visit notes existing in the current database. The module does not automatically run its analysis on these documents. Once installed, it will run the analysis on all Visit Notes that are subsequently submitted. Our module provides the ability to retroactively run the text analysis on Visit Notes currently in the Database.

To analyze all Visit Notes in the database:

1. Navigate to the "Manage Visit Notes Analysis" page from the Administration Page.

Figure 17: Re-running Visit Note analysis

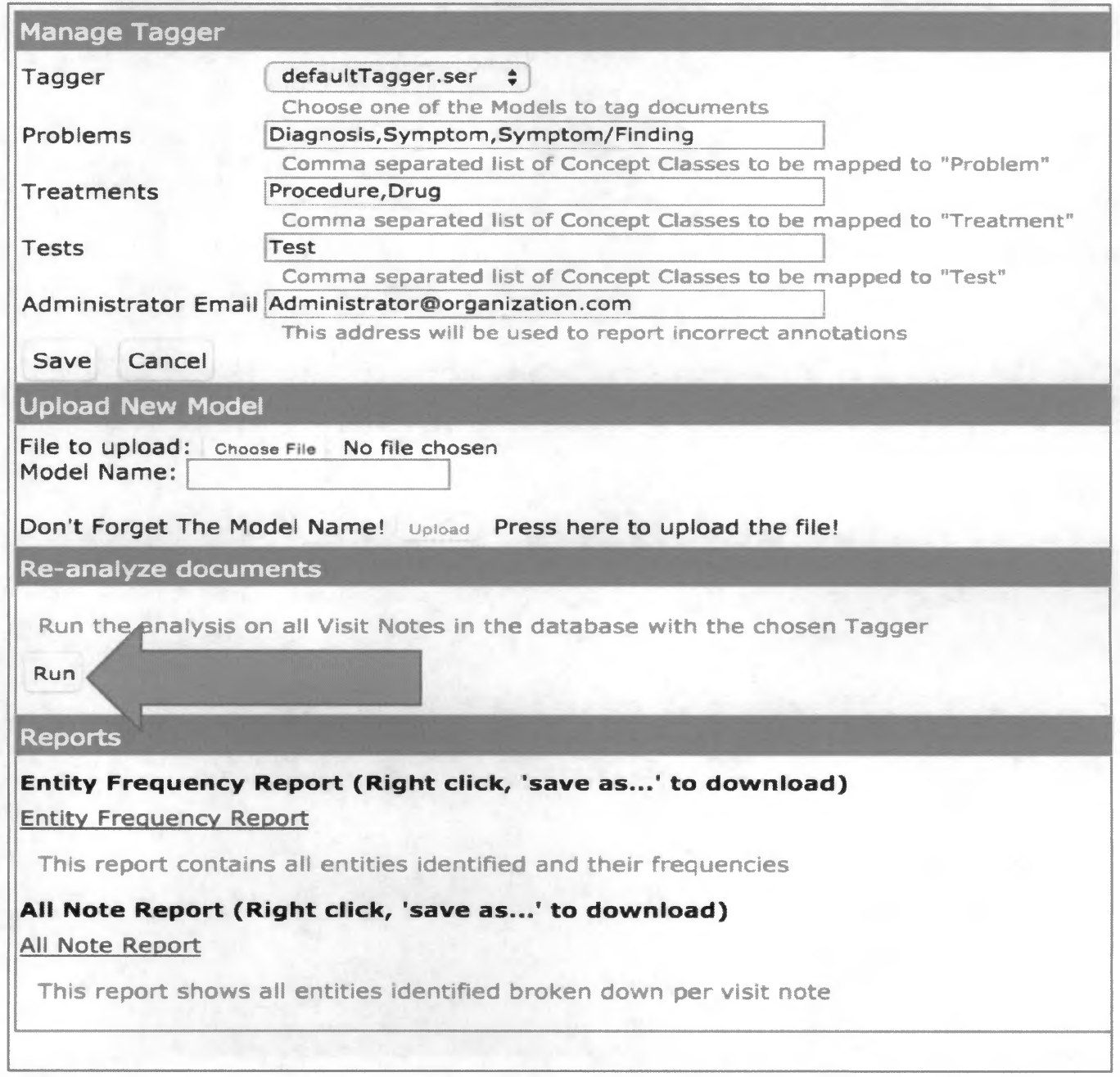


2. Select "Run" to run the text analysis on all Visit Notes in the database, figure 17. NOTE: As there may be a very large number of Visit Notes in the database, this job may take a while and consume considerable resources. We recommend running this job during low demand, low traffic periods.

3. Results from this analysis will now be visible through the "Visit Notes Analysis" action on the patient dashboard, figures 18 and 19.

Figure 18: Patient Dashboard shows how to find the Visit Notes Analysis module

\begin{tabular}{|c|c|c|c|}
\hline OpenMRS & & admin & Inpatient Ward - Logout [5 \\
\hline \multicolumn{4}{|l|}{ ศ J John D Patient } \\
\hline $\begin{array}{l}\text { John D Patient Male } 40 \text { year(s) }(01, j a n .197 \\
\text { Given Middle Fannly Name }\end{array}$ & 75) Edil Show Contact info - & & Patient id \\
\hline \multicolumn{4}{|c|}{ Active Visit - 29 Jul 2015 02:42 PM Outpatient } \\
\hline K DIAGNOSIS & VISITS & $\stackrel{c}{e}$ & \\
\hline Anaemia & Ioday & Active-Outparient & Current Visit Actions \\
\hline VITALS & allergies & 2 & $\begin{array}{l}\text { (1) End Visit } \\
\text { y Visit Note }\end{array}$ \\
\hline None & Unknown & & Capture Vitals \\
\hline APPOINTMENTS & & & General Actions \\
\hline None & & & $\begin{array}{l}+ \text { Add Past Visit } \\
\text { Merge Visits } \\
\text { Chart Search } \\
\text { Visit Notes Analysis }\end{array}$ \\
\hline
\end{tabular}


Figure 19: Results of NER analysis visible from the Visit Notes Analysis page

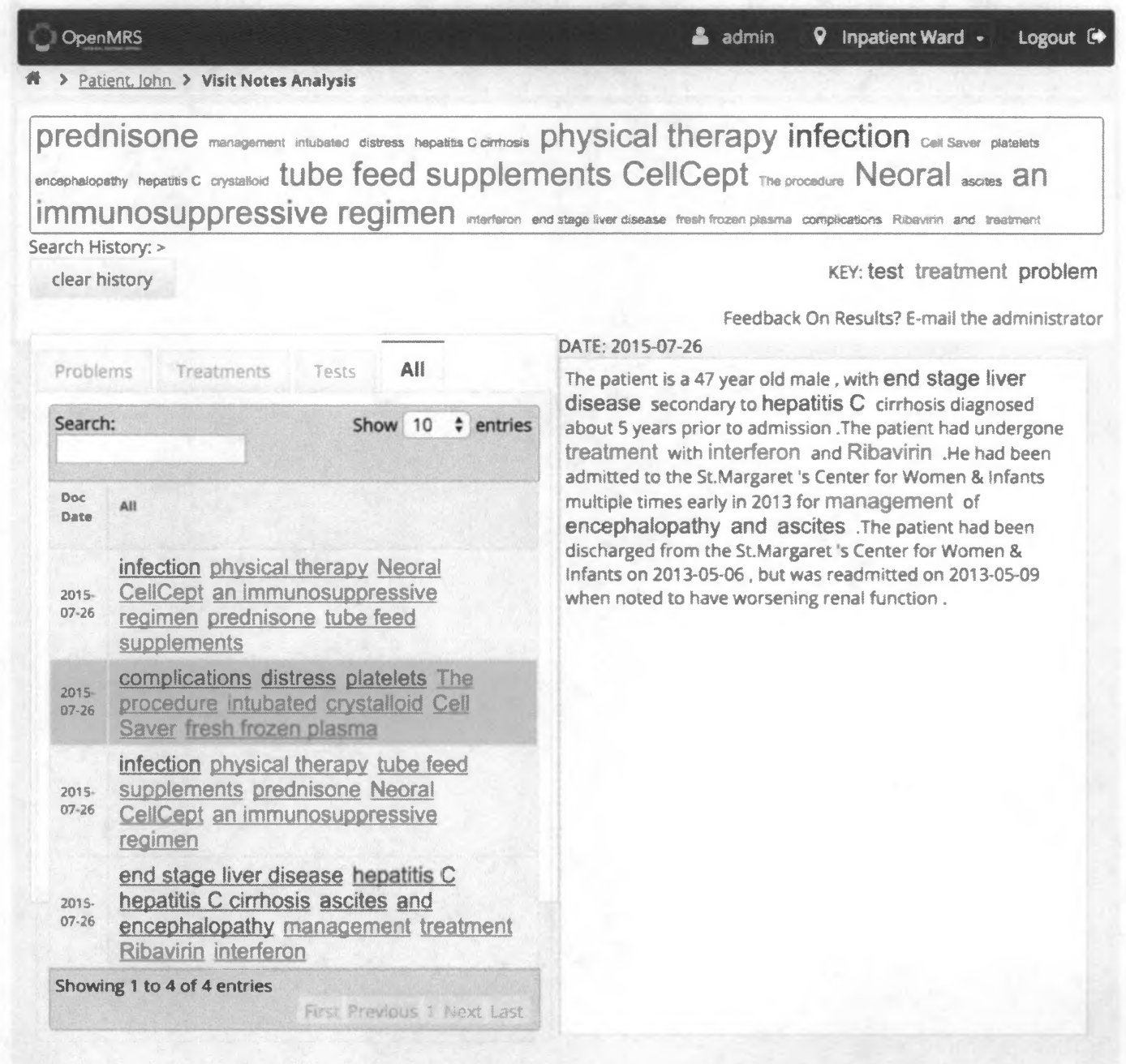

\subsection{Adding a New Model}

The companion application "Visit Note Annotator and Trainer" allows the user to build a new model to improve the module's ability to recognize medical entities. See section 6 of this guide for instructions on building a new model. Once the Trainer has built the model file, follow these steps to upload the model into the module. 
Uploading a new Model:

1. Navigate to the "manage Visit Notes Analysis" page from the OpenMRS Administration page.

2. Select "Choose File" and find the model file on your local file system, figure 20.

Figure 20: Uploading a new model

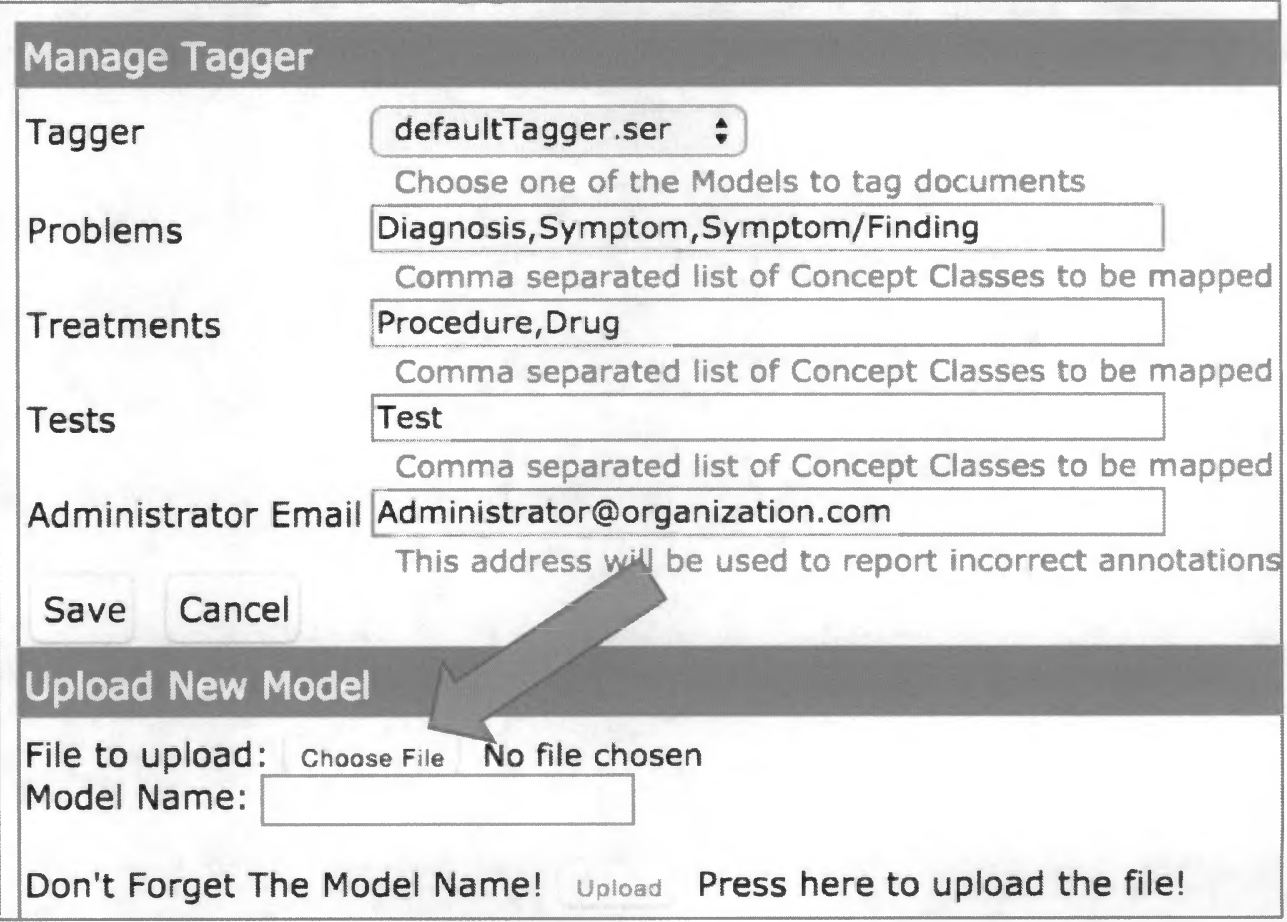

3. Input a name for your model, figure 21 .

4. Select "upload," figure 21.

5. The new model is now uploaded into the module and can be chosen for tagging in the future 


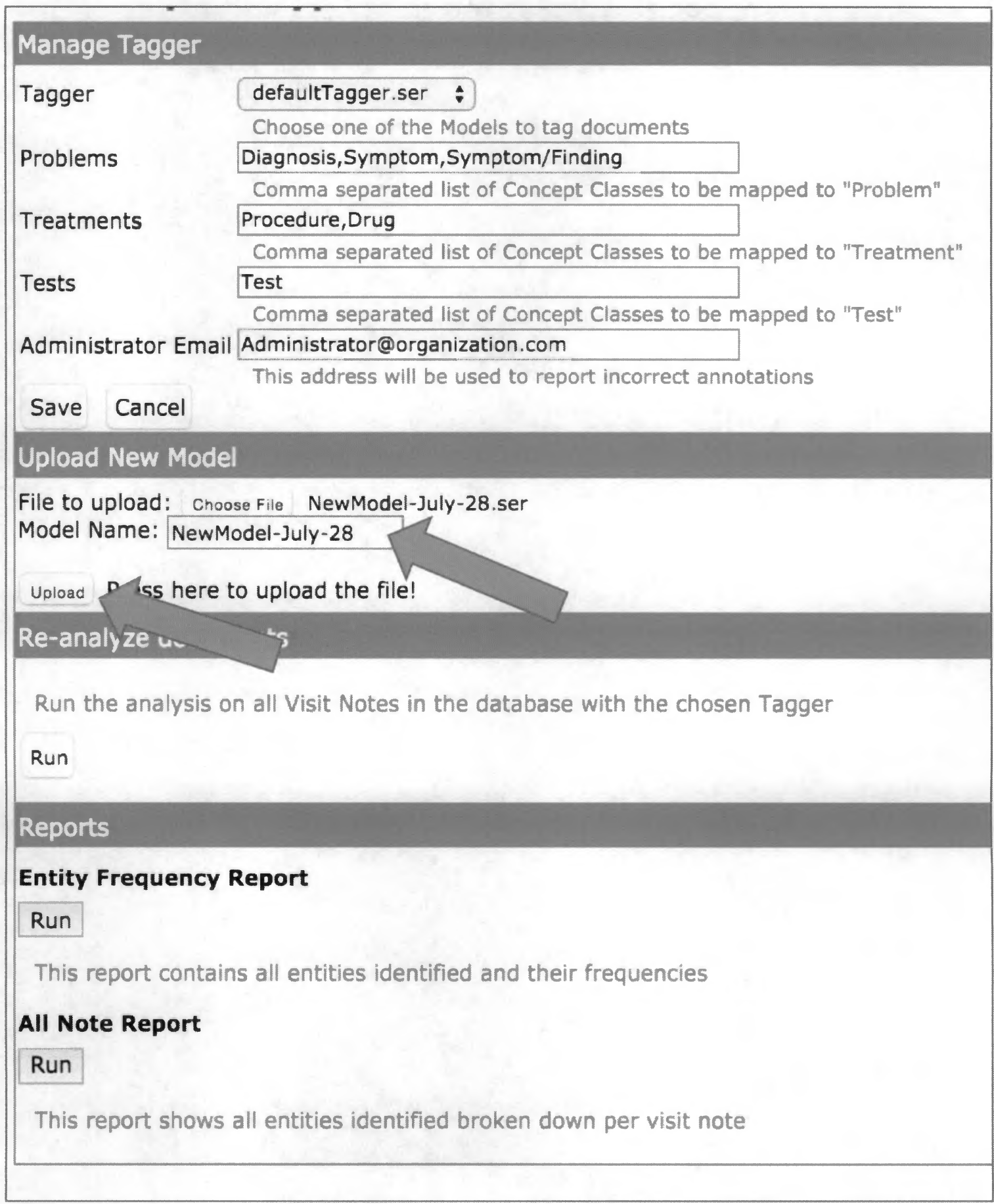




\subsection{Choosing a Model}

This module is distributed with a default tagging model and allows for new models to be uploaded. To configure which tagger your module will use, follow these instructions

Choosing a Tagger:

1. Navigate to the Manage Visit Notes Analysis page. 
Figure 22: choosing a tagger from the dropdown

\begin{tabular}{|l|l|} 
Manage Tagger & NewModel-July-28 \\
Problems & Choose one of the Models to ag documents \\
Tiagnosis,Symptom,Symptom/Finding & Comma separated list of Concept Classes to be mapped to "Problem" \\
Treatments & Procedure,Drug \\
Tests & Comma separated list of Concept Classes to be mapped to "Treatment" \\
Administrator Email & Administrator@organization.com \\
Save Cancel & This address will be used to report incorrect annotations
\end{tabular}

Figure 23: Select your tagger

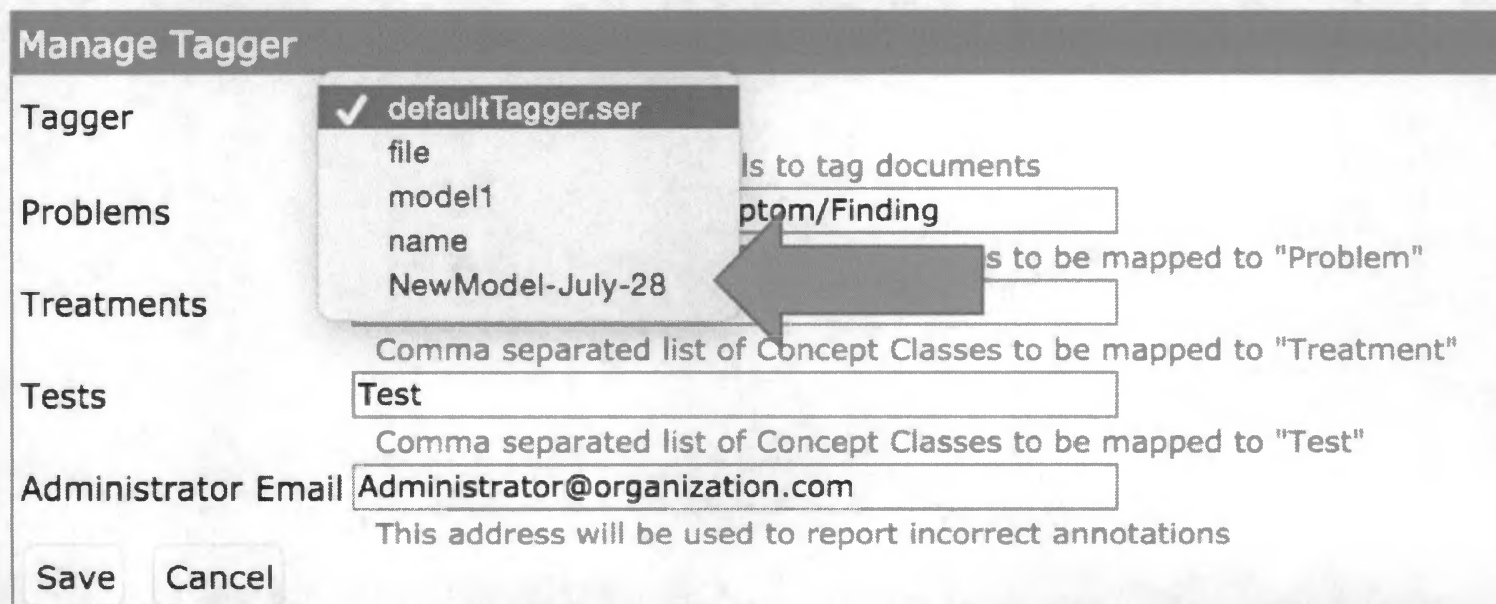

2. select the dropdown list under "Manage Tagger," figures 22 and 23.

3. Save this new setting by clicking the "save" button, figure 24 .

4. NewModel-July-28 will now be used to tag new Visit Notes.

5. Optionally, the admin can now follow the steps in section 3.2 to re-analyze the existing corpus of visit notes using the new model. All old models are saved (see the dropdown menu in figure 23) and can be chosen for use by following the steps in this section. 
Figure 24: Save this new setting

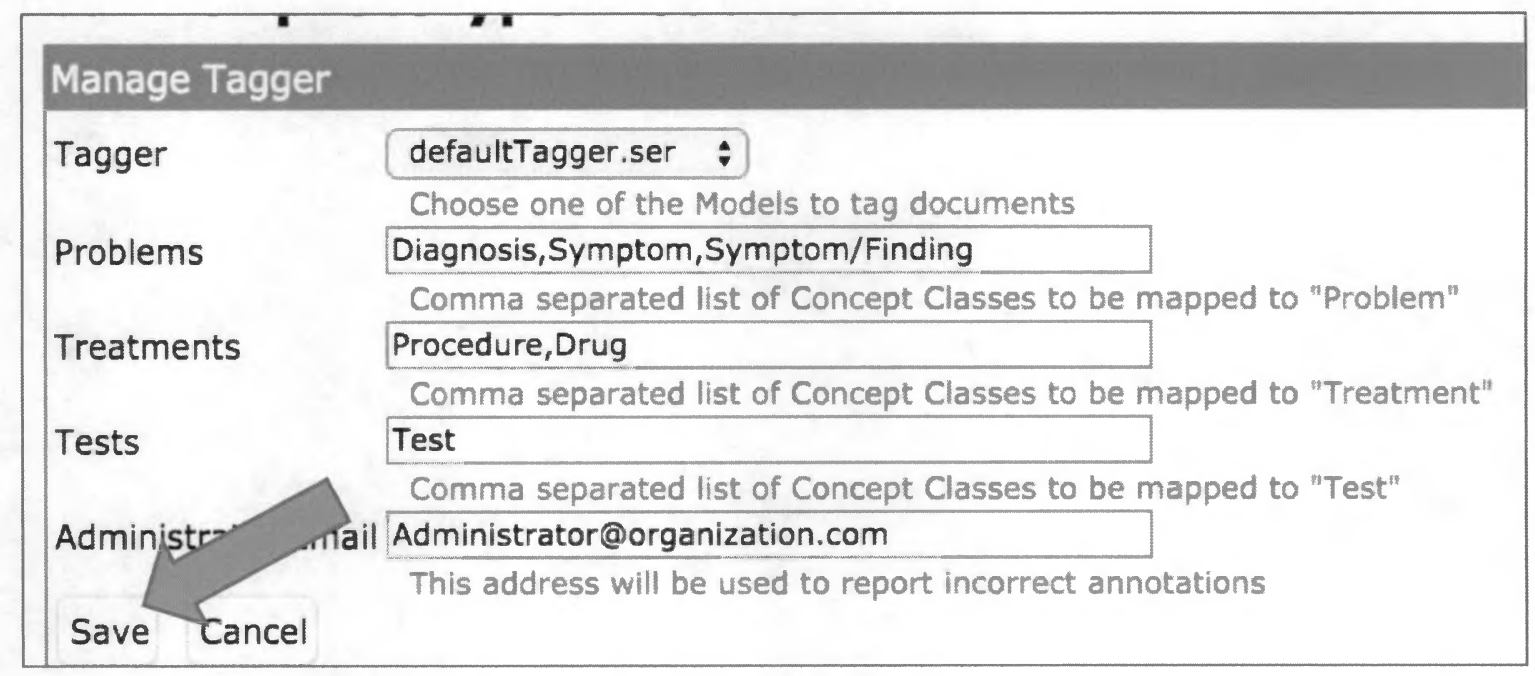

\subsection{Running Reports}

The Visit Notes Analysis module allows you to run two reports at this time. The Entity Frequency Report and the All Notes Report.

The Entity Frequency Report generates a csv file with all entities and their entity type along with the number of occurrences of that entity in the entire visit note corpus. Output is in the format:

Entity, type, frequency

For example:

$$
\begin{aligned}
& \text { Nausea, problem, } 5 \\
& \text { cd4, test, } 4
\end{aligned}
$$

The All Notes Report generates a csv that shows all notes analyzed in the system and the entities in them. This note shows one note per line in the format: 
entities\&\&types is a vertical bar separated list of all entities found in the note DocID is a unique document identifier. An example line in this report:

$3,1,2015-07-2914: 42: 48.0$, nausea\&\&problem|vomiting\&\&problem

To run the reports, follow these instructions;

Running Reports:

1. Navigate to the Manage Visit Note Analysis page

2. Find the section labeled "Reports," figure 25.

3. This section contains links for the Entity Frequency Report and the All Notes Report 
Figure 25: Running Reports

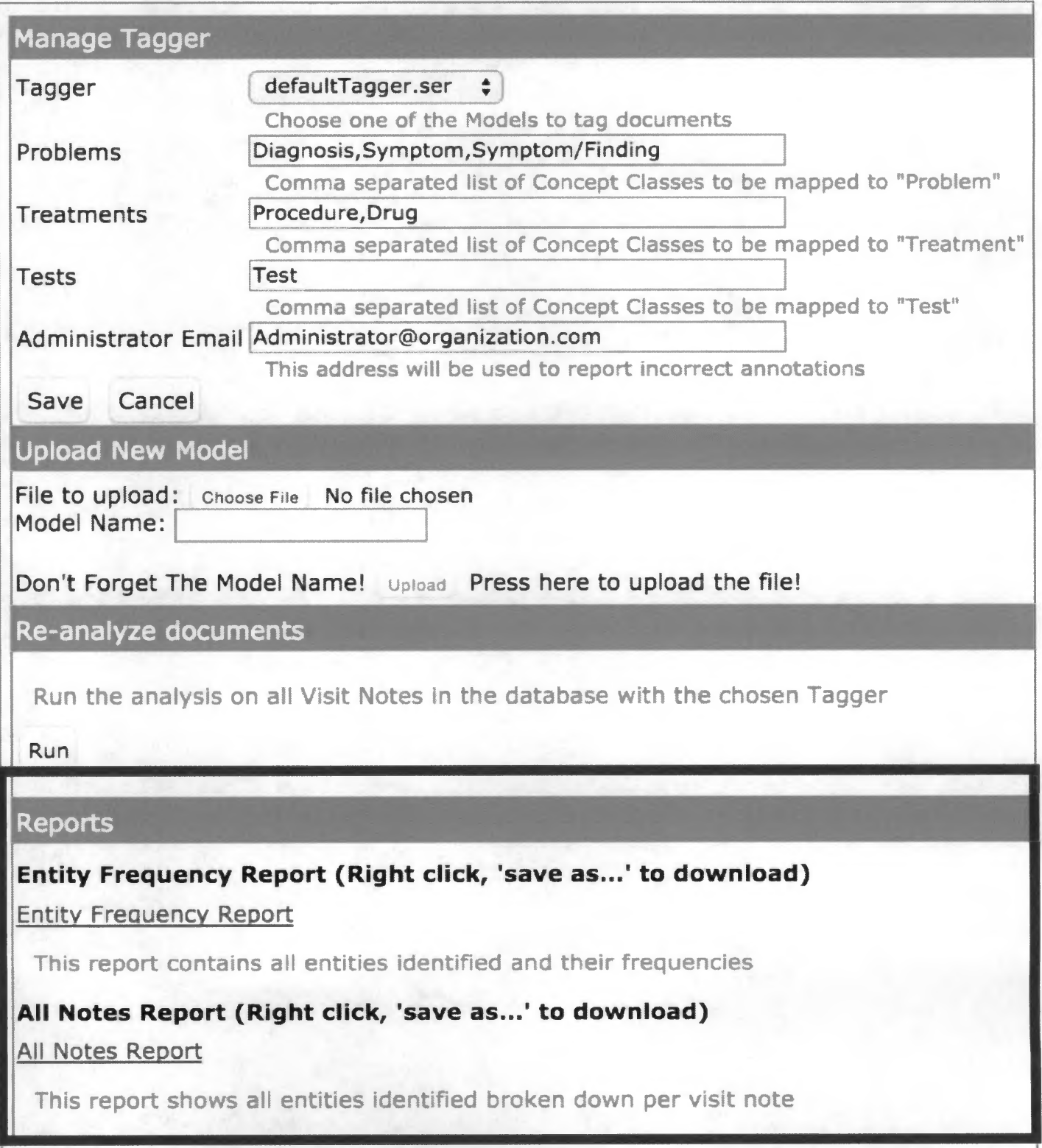

4. Clicking on the link will render the report in the browser, figures 26 and 27. 


\section{Reports}

Entity Frequency Report (Right click, 'save as...' to download) Entity Frequency Report

This report contains all entities identified and their frequencies

\section{All Notes Report (Right click, 'save as...' to download)} All Notes Report

This report shows all entities identified broken down per visit note

Figure 27: Entity Frequency Report

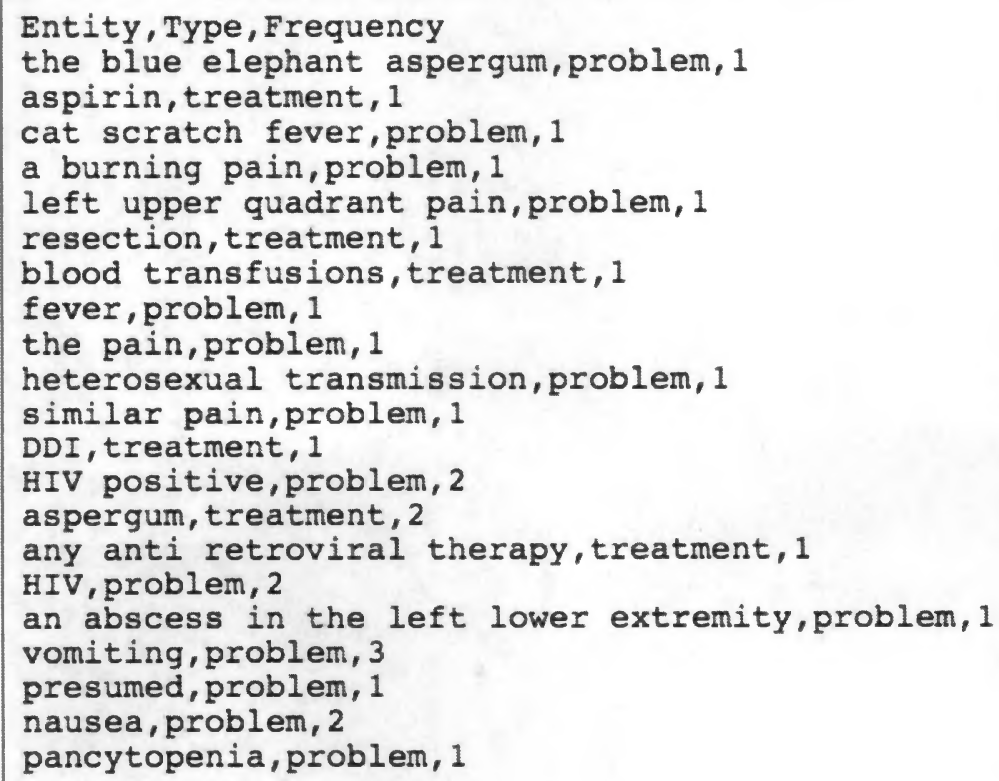

5. Right click on the link and select "Save as..." to download the report to your local machine. 


\section{Visit Note Annotator User Interface}

The UI consists of 6 major components. They are labeled in figure 28 , followed by a brief description. Usage details are provided in Section 5. The components are as follows:

1. Help

2. Populate

3. Visit Notes List

4. Visit Note Annotation Editor

5. Change Log

6. Controls

Figure 28: Application with UI components labeled

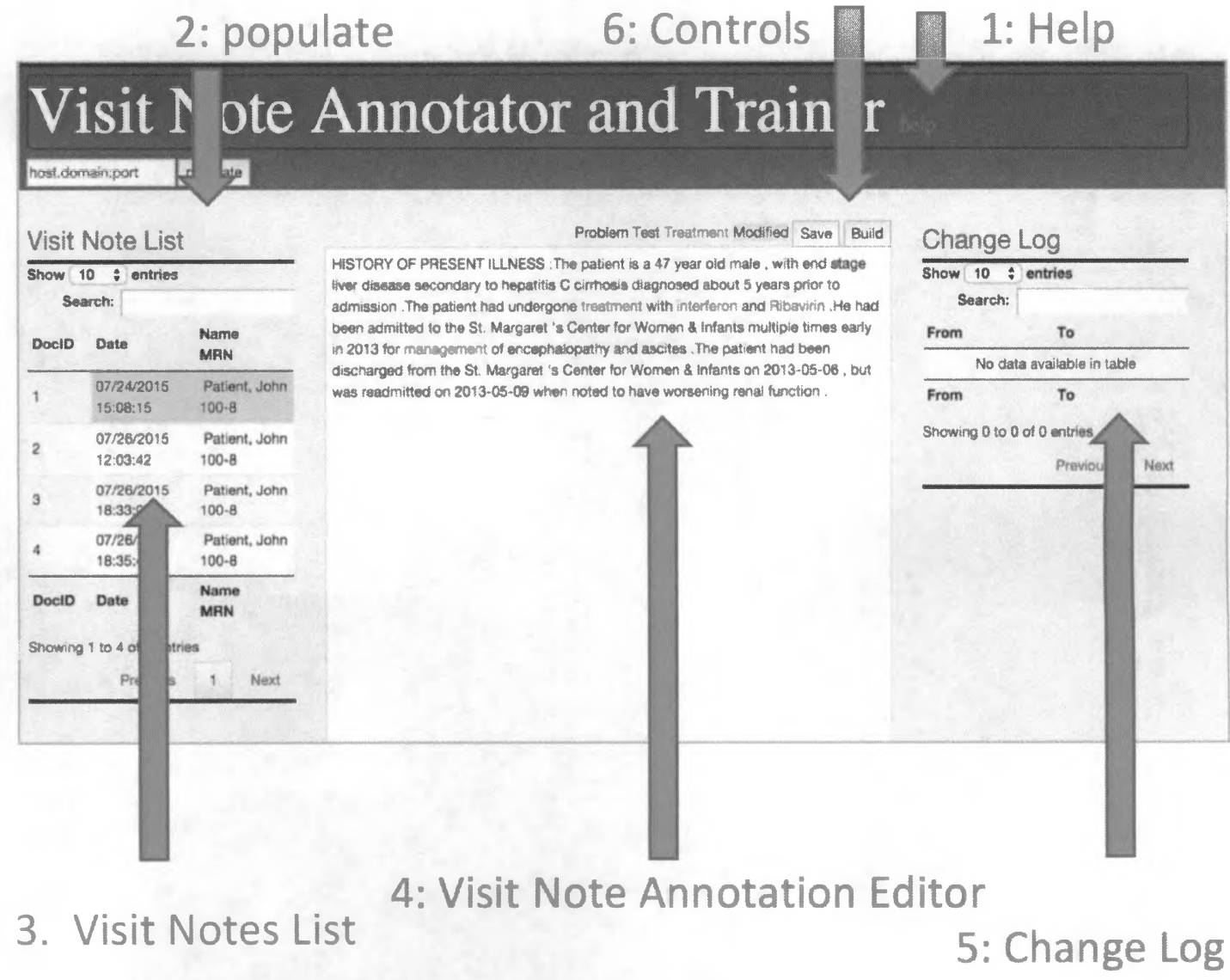




\subsection{Help}

Clicking on the help button shows six popover tool tips to assist the user. The next time the user clicks the mouse, these popovers disappear. Figure 29 shows this.

Fiqure 29: Application with help tool tips showing

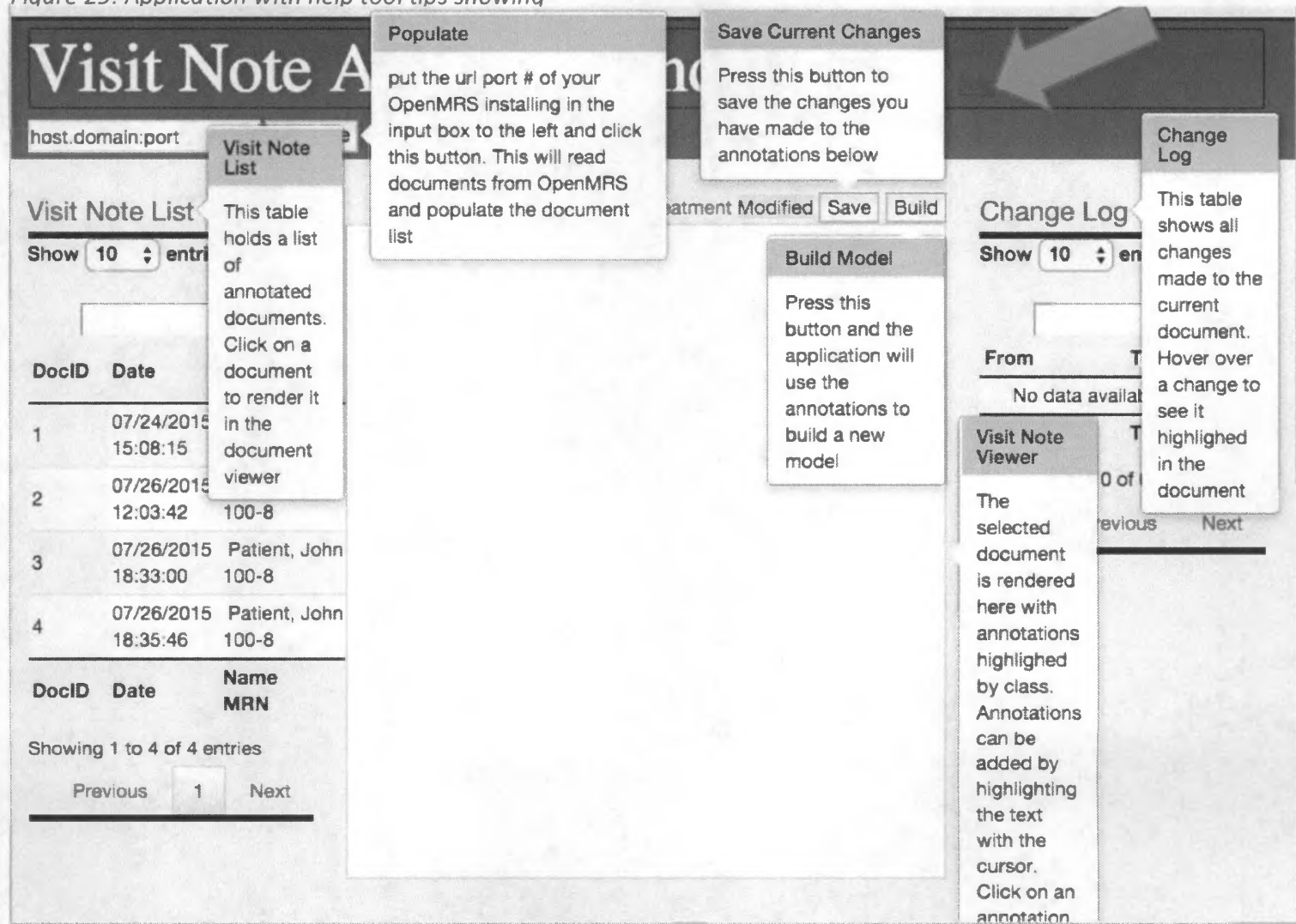

\subsection{Populate}

The Populate component is used to communicate with the OpenMRS installation to read in Visit Notes. More details are provided in section 5. 


\subsection{Visit Notes List}

The Visit Notes list shows the visit notes that were imported from OpenMRS. This list is used to select a document to annotate. Three data points are provided for each Visit Note: The DocID, The date/time of the note, Patient name/Medical Record Number. When a note is selected for editing, it will be highlighted in the Visit Note List

\subsection{Visit Note Annotation Editor}

The editor allows the user to add new annotations to the Visit Note text as well as correct or delete existing annotations.

\subsection{Change log}

The Change Log keeps a record of changes made to the current Visit Note being displayed with the option to undue the changes.

\subsection{Controls}

The controls allow the user to save current changes as well as build the new model based on all documents an all annotations currently in the app.

\section{Visit Note Annotator Usage}

\subsection{Import Data from OpenMRS}

This application does not read from the OpenMRS database directly. Once the Visit Notes Analaysis module is installed, an HTTP URL is exposed when an administrator navigates to the Manage Visit Notes Analysis page. The web app then uses this endpoint URL to request the Visit Note Data from OpenMRS. 
Steps to populate the Visit Notes List are the following:

1. Administrator navigates to Manage Visit Notes Analysis page within OpenMRS

Fiqure 30: OpenMRS Administration paqe with Manaqe Visit Note Analysis hiahliahted

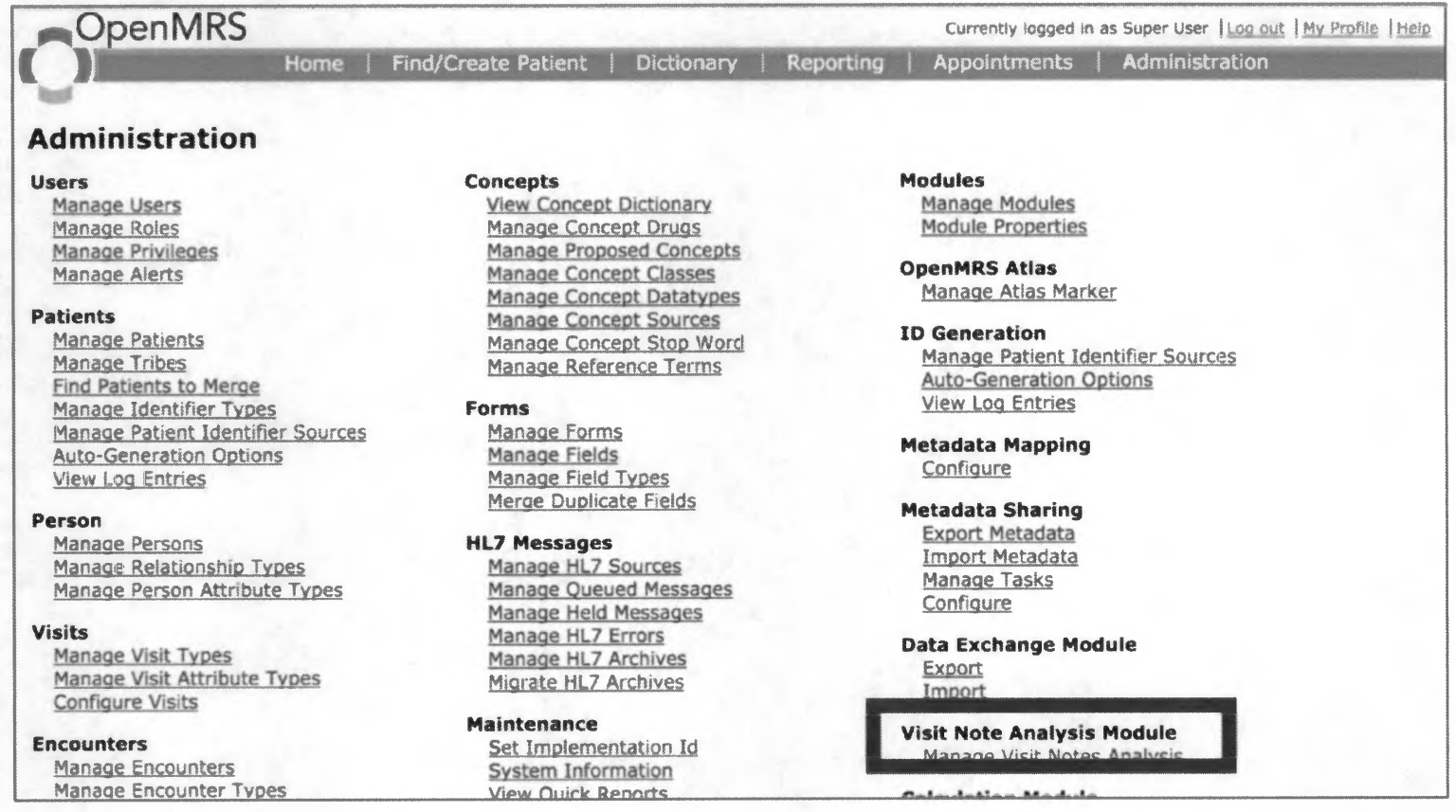




\section{Once here, the URL endpoint can be accessed by the application.}

Figure 31: Manage Visit Notes Analysis page, with URL:port\# of installation highlighted

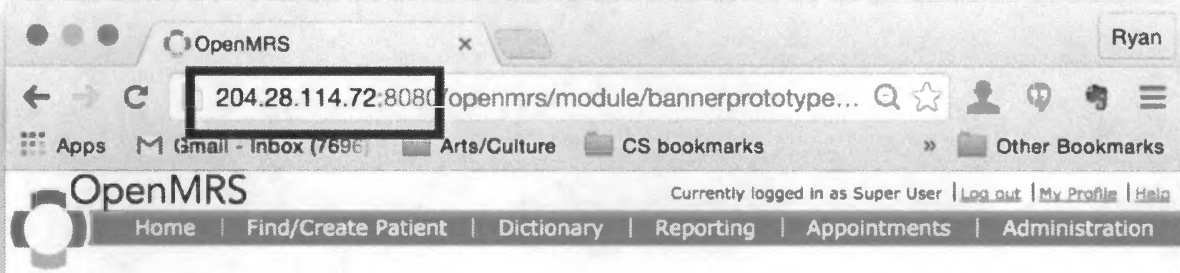

Admin / Manage module

\section{banner prototype Module}

\section{Manage Tagger}

Tagger default Tagger ser

Choose one of the Models to tag documents

Probiems Diagnasis, Symptom, Symptom/Finding

Comma separated list of Concept Classes to be mapped to "Problem"

Treatments Procedure, Drug

Tests

Comma separated list of Concept Classes to be mapped to "Treatment"

Administrator Email Administrator *organization.com

This address witl be used to neport incorrect annotations
Ad

Save Cancel

\section{Upload New Model}

File to upload: Chooso Fia No fie chosen

Model Name:

Don't Forget The Model Name! ysiond Press here to upload the file

Re-analyze documents

Run the analysis on all Visit Notes in the database with the chosen Tagger

Run

\section{Reports}

Entity Frequency Report (Right click, 'save as...' to download)

Entity Freouency Report

This repart contains all entities identified and their frequencies

All Notes Report (Right click, 'save as...' to download)

All Notes Report

This report shows all entities identified broken down per visit note 
3. Enter the URL and port number into the input box next to the "populate" button in the web applications. The URL and Port number are highlighted in figure 31.

Figure 32: Visit Note Annotator and Trainer app with OpenMRS URL:Port\# in "populate" input

\section{OpenMRS Visit Note Analysis Module Companio}

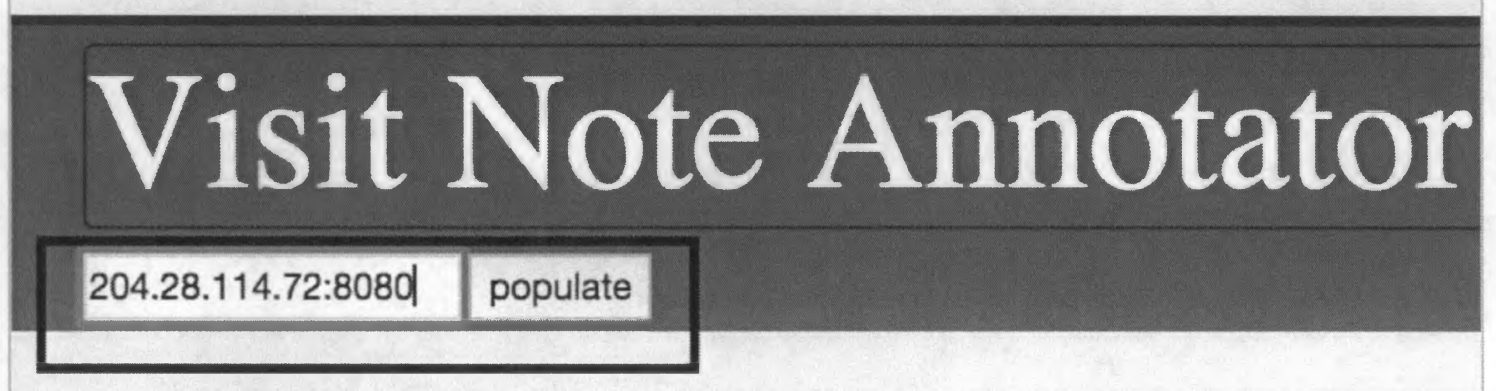

4. Click the "populate" button. 
5. The documents from OpenMRS will now appear in the Visit Note List, figure 33.

Fiaure 32: visit notes bopulated from ODenMRS

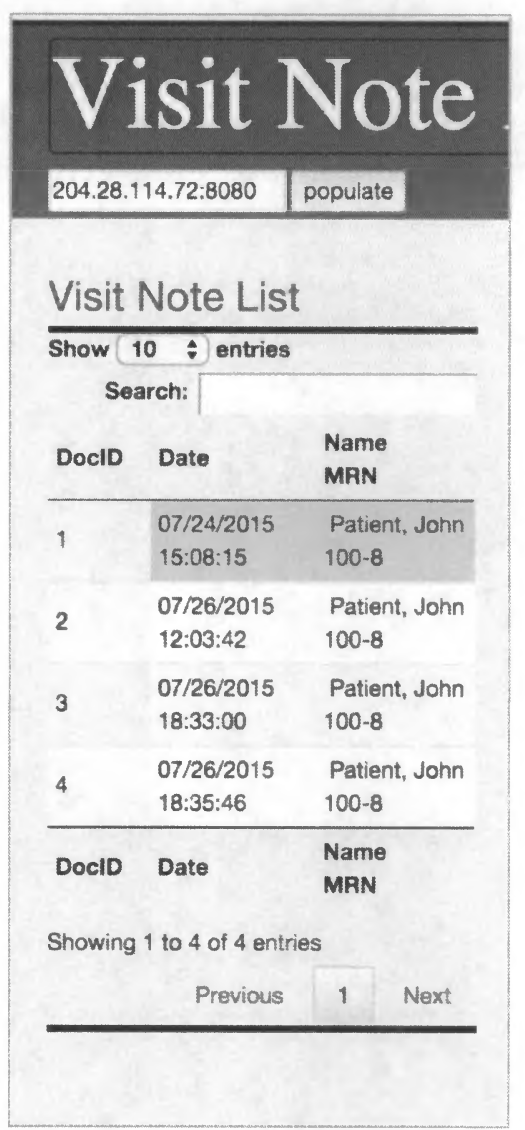




\subsection{Selecting a Visit Note}

The user can search for a specific visit note with the Search Box or file through them sequentially with the "previous" and "next" buttons. Once the desired document is in view, clicking on the document will render it in the Editor.

Figure 34: Document selected with text and annotations rendered

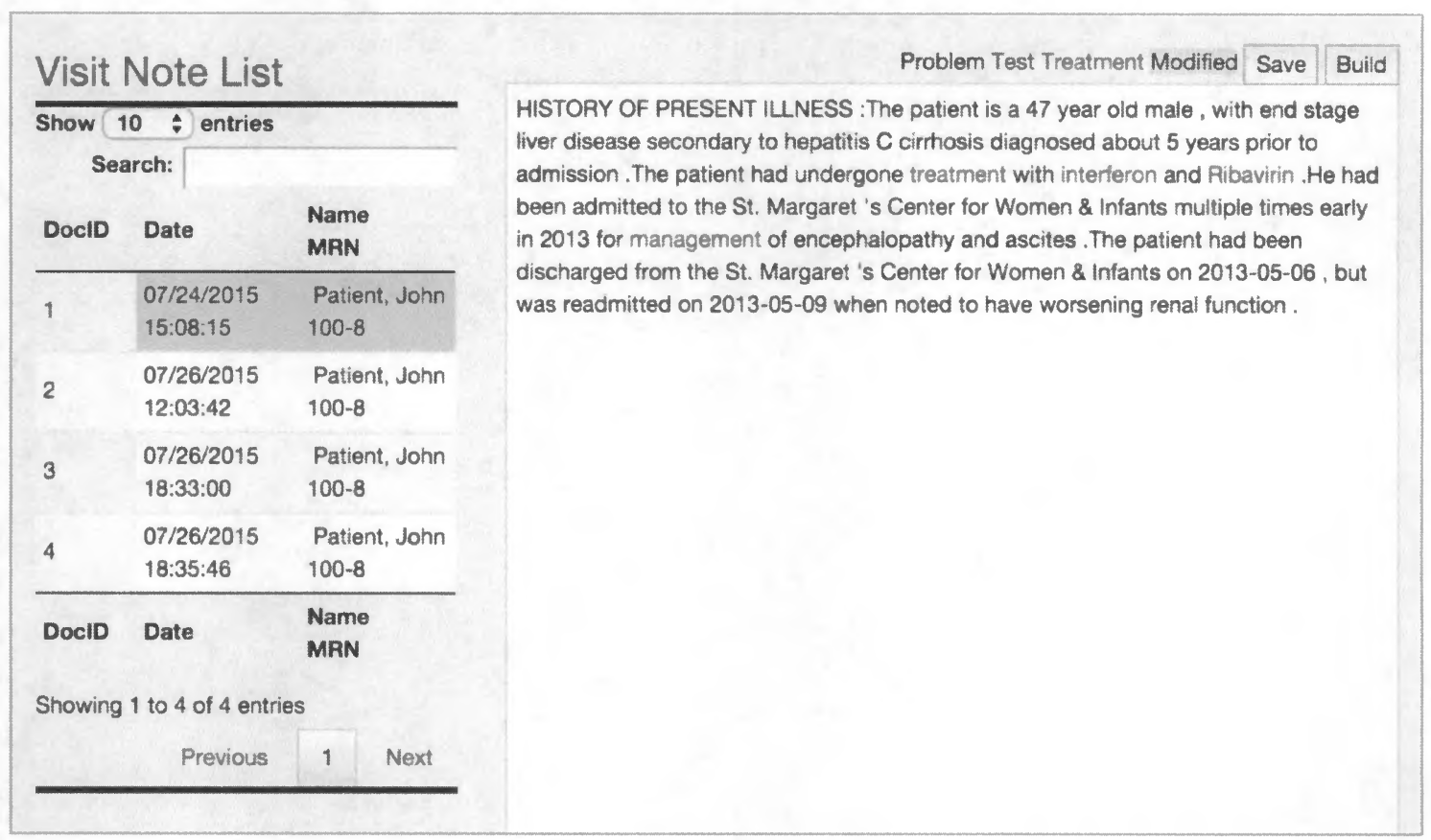

\subsection{Editing Visit Note Annotations}

To edit the annotations of a Visit Note, the user can click on an annotation to either change the annotation type, or remove it from the annotations list, figure 35. 
Fiqure 35: Editina annotations. hepatitis C cirrhosis selected

Problem Test Treatment Modified Save Build

HISTORY OF PRESENT ILLNESS :The patient is a 47 year old male, with end stage liver disease secondary to hepatitis $C$ cirrhosis diagnosed about 5 years prior to admission. The patient had undergone treatment with interferon and Ribavirin. He had been admitted to the St. Margaret 's Center for Women \& Infants multiple times early in 2013 for management of encephalopathy and ascites. The patient had been discharged from the St. Margaret 's Center for Women \& Infants on 2013-05-06, but was readmitted on 2013-05-09 when noted to have worsening renal function.

\section{Choose Concept Type For}

hepatitis C cirrhosis

Problem Treatment Test None

Any changes made by the user will be reflected in the Visit Note rendering as well as the

Problem Test Treatment Modified Save Build

HISTORY OF PRESENT ILLNESS :The patient is a 47 year old male, with end stage liver disease secondary to hepatitis $\mathrm{C}$ cirrhosis diagnosed about 5 years prior to admission. The patient had undergone treatment with interferon and Ribavirin. He had been admitted to the St. Margaret 's Center for Women \& Infants multiple times early in 2013 for management of encephalopathy and ascites. The patient had been discharged from the St. Margaret 's Center for Women \& Infants on 2013-05-06, but was readmitted on 2013-05-09 when noted to have worsening renal function .

Change Log
\begin{tabular}{rll} 
Show $10 \div$ entries \\
Search: & & \\
From & To & \\
\hline $\begin{array}{lll}\text { hepatitis C } & \text { hepatitis C } & \text { undo } \\
\text { cirrhosis } & \text { cirnosis } & \text { To }\end{array}$ \\
\hline From & To
\end{tabular}

Showing 1 to 1 of 1 entries

Previous 
Change Log. Here the user changed the concept type of hepatitis C cirrhosis from "problem" to "treatment." See figure 36.

The user can add a new annotation by highlighting the text. Once a text is highlighted, the same entity-type selection box will appear, allowing the user to select the annotation type, figure 37.

Fiaure 37: User hiahliahed "worsenina renal function" and is oromoted to choose an entitv tvoe

Problem Test Treatment Modified Save Build

HISTORY OF PRESENT ILLNESS :The patient is a 47 year old male, with end stage liver disease secondary to hepatitis $\mathrm{C}$ cirrhosis diagnosed about 5 years prior to admission. The patient had undergone treatment with interferon and Ribavirin. He had been admitted to the St. Margaret 's Center for Women \& Infants multiple times early in 2013 for management of encephalopathy and ascites. The patient had been discharged from the St. Margaret 's Center for Women \& Infants on 2013-05-06, but was readmitted on 2013-05-09 when noted to have worsening renal function.

\section{Choose Concept Type For}

worsening renal function

Problem Treatment Test None




\subsection{Reviewing Edits}

The user can use the change log to review and undo annotation edits that have been made. Hovering the mouse over a change highlights it in the text, figure 38.

Figure 38: User hovers mouse over Change Log entry, corresponding entity is highlighted

Problem Test Treatment Modified Save Build

HISTORY OF PRESENT ILLNESS : The patient is a 47 year old male, with end stage liver disease secondary to hepatitis $C$ cirrhosis diagnosed about 5 years prior to admission. The patient had undergone treatment with interferon and Ribavirin. He had been admitted to the St. Margaret 's Center for Women \& Infants multiple times early in $\mathbf{2 0 1 3}$ for management of encephalopathy and ascites. The patient had been discharged from the St. Margaret 's Center for Women \& Infants on 2013-05-06, but was readmitted on 2013-05-09 when noted to have worsening renal function .

\begin{tabular}{|c|c|c|}
\hline \multirow{2}{*}{\multicolumn{3}{|c|}{$\begin{array}{c}\text { Show } 10 \quad \\
\text { Search: }\end{array}$}} \\
\hline & & \\
\hline \multicolumn{3}{|l|}{ From } \\
\hline $\begin{array}{l}\text { hepatitis C } \\
\text { cirnosis }\end{array}$ & $\begin{array}{l}\text { hepatitis } \\
\text { cirrhosis }\end{array}$ & undo \\
\hline worcening renal & worsening & \\
\hline function & $\begin{array}{l}\text { renal } \\
\text { function }\end{array}$ & undo \\
\hline From & To & \\
\hline
\end{tabular}

Selecting the "undo" button will reverse the corresponding edit change.

Here the user has selected "undo" and "hepatitis C cirrhosis" has reverted from the Figure 39: User selects "undo" and hepatitis C cirrhosis is revered from treatment back to test. The change log entry is deleted.

Problem Test Treatment Modified Save Build

HISTORY OF PRESENT ILLNESS :The patient is a 47 year old male, with end stage liver disease secondary to hepatitis $\mathrm{C}$ cirnosis diagnosed about 5 years prior to admission. The patient had undergone treatm fith interferon and Ribavirin. He had been admitted to the St. Margaret 's Center fo. 0 \& Infants multiple times early in 2013 for management of encephalopathy and as. The patient had been discharged from the St. Margaret 's Center for Women. was readmitted on 2013-05-09 when noted to have worsen renal function

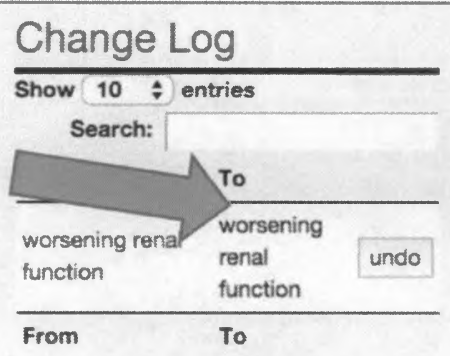

"treatment" to "problem" and the entry has been removed from the Change Log. Figure 39 


\subsection{Save Annotations}

When the user is satisfied with the changes made to the text, they can save the changes by clicking on the save button. If the user navigates to another note without saving, they will be prompted to save.

Figure 40: User has saved annotations.

\begin{tabular}{l} 
Problem Test Treatment Modified Save \\
$\begin{array}{l}\text { HISTORY OF PRESENT ILLNESS :The patient is a } 47 \text { year old male, with end } \\
\text { liver disease secondary to hepatitis } \mathrm{C} \text { cirrhosis diagnosed about } 5 \text { years prior to } \\
\text { admission. The patient had undergone treatment with interferon and Ribavirin. He had } \\
\text { been admitted to the St. Margaret 's Center for Women \& Infants multiple times early } \\
\text { in } 2013 \text { for management of encephalopathy and ascites. The patient had been } \\
\text { discharged from the St. Margaret 's Center for Women \& Infants on 2013-05-06, but } \\
\text { was readmitted on 2013-05-09 when noted to have worsening renal function. }\end{array}$ \\
\hline
\end{tabular}

\subsection{Build Model}

When the user is satisfied with all the annotations on all the documents under scrutiny. They can use the documents and annotations to build a new model. Clicking on the build button will begin model computation, figure 41 .

Fiqure 41: Select "build" to use text and annotations to compute new model.

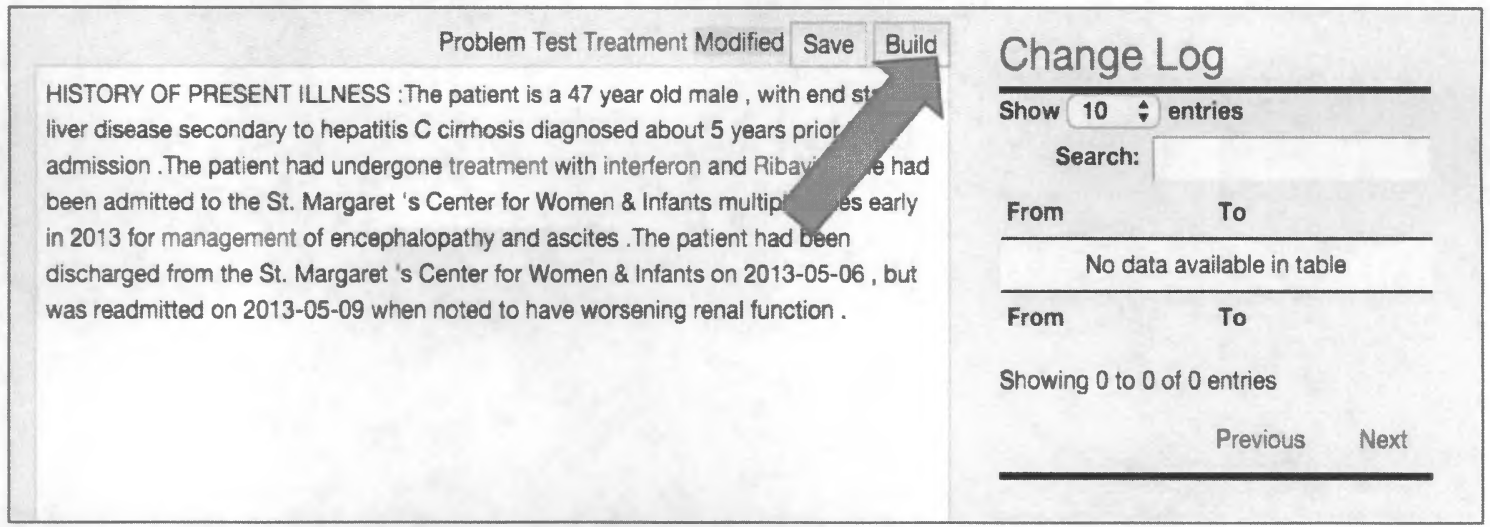

NOTE: This may take some time, depending on the number of documents 


\subsection{Export Model}

When the training process is complete, the user will be notified and a "download" button will appear. Clicking the download button will allow the user to download the new model.

Figure 42: The model has been computed and is available for download.

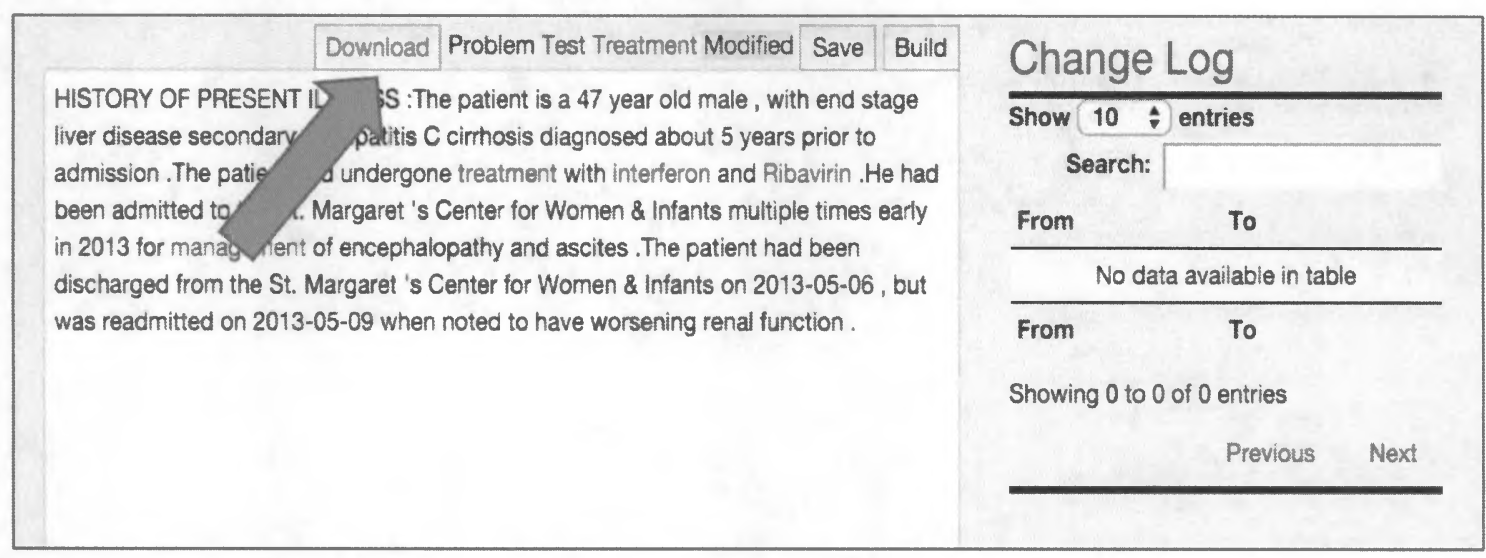

The user can now refer to section 3 of this guide for instructions on how to load this model into OpenMRS and use it for tagging Visit Note text. 
Appendix 3:

Visit Notes Analysis

Developer's Guide 

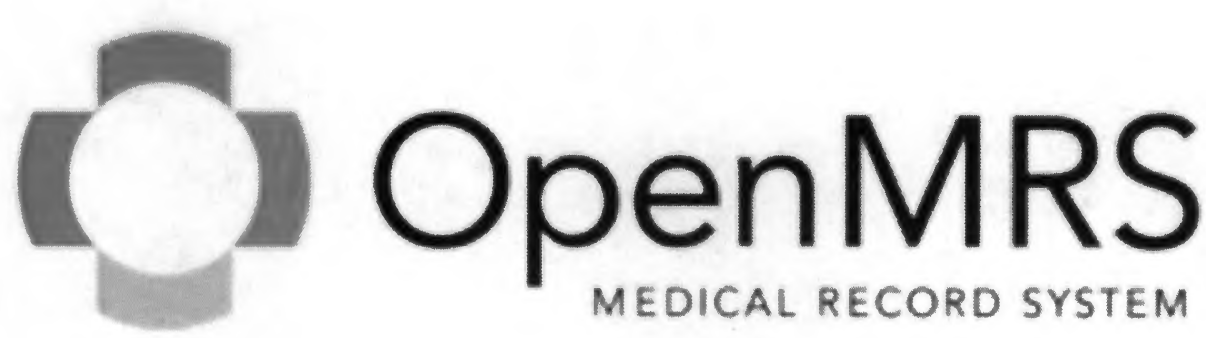

\section{Visit Note Analysis Module Developer's Guide}

Ryan Eshleman

Advisors: Dr. Barry Levine

Dr. Hui Yang 
Table of Contents

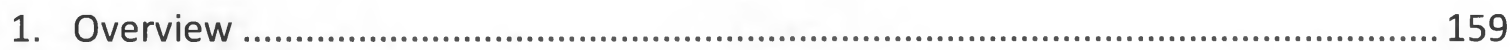

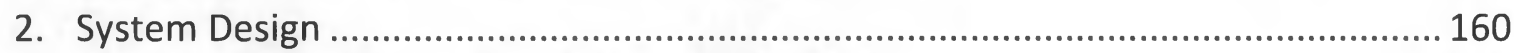

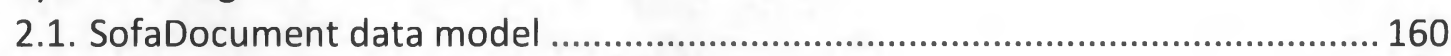

2.2. Named Entity Recognition Algorithm........................................................ 162

2.3. Visit Note Processing with AOP ................................................................. 163

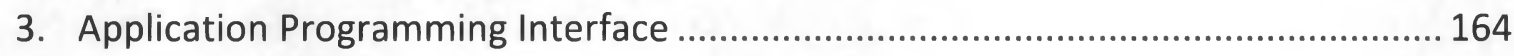

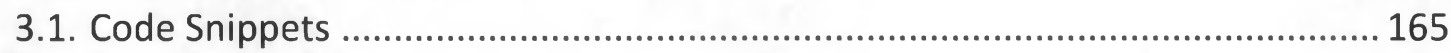

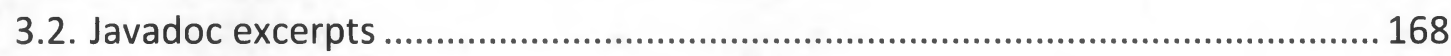

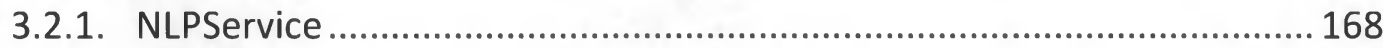

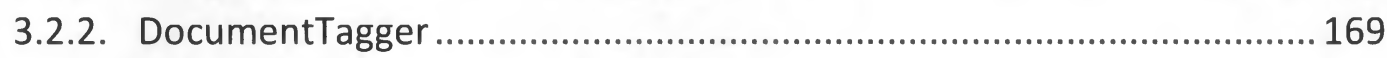

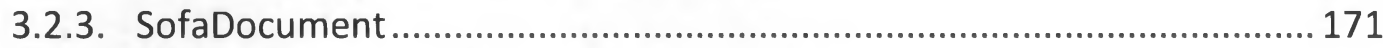

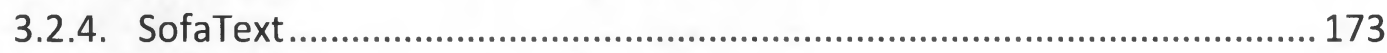

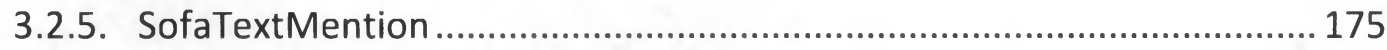

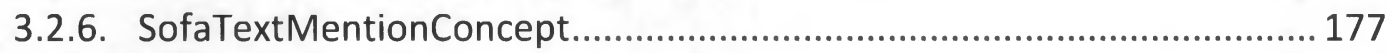

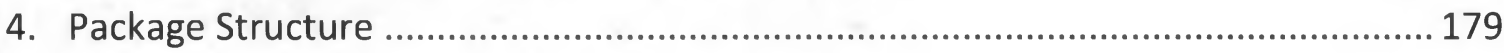




\section{Overview}

The Visit Notes Analysis module was built to provide Named Entity Recognition (NER) capabilities to OpenMRS to help the system extract more information from Visit Note text as well as other potential sources of unstructured text.

Part of the challenge of enhancing OpenMRS's ability to analyze plain text is how can the user gain the most value out of the analysis. Our module provides one use, summarizing and visualizing the information in a patient note, however we understand that there are many possible uses of NER.

It is with this understanding that we write the Developer's Guide in order to provide the future developers with the background necessary to either continue and refine the development of this module, or leverage the NER API provided by this module in order to build new and better projects.

In this guide you will find three sections

1. System Design: A high level description of the three main components working under the hood to carry out the module's NER functions.

2. Application Programming Interface: Details and Examples of the main Java classes used to support the NER functionality

3. Package Structure: A diagram of the package structure with brief descriptions of each package. 


\section{System Design}

There are three major system design components that a developer should be familiar with in order to continue development with this module. Those components are:

1. The SofaDocument data model

2. The Named Entity Recognition algorithm

3. Processing Visit Notes with Aspect Oriented Programming

\subsection{SofaDocument data model}

The module adds four database tables to the OpenMRS datamodel corresponding to the SofaDocument data hierarchy ('sofa' is shorthand for Subject of Analysis). The data stored in these tables is accessible via the service layer through the service class NLPService. The data model is shown in figure 1.

Figure 1: SofaDocument Data Model

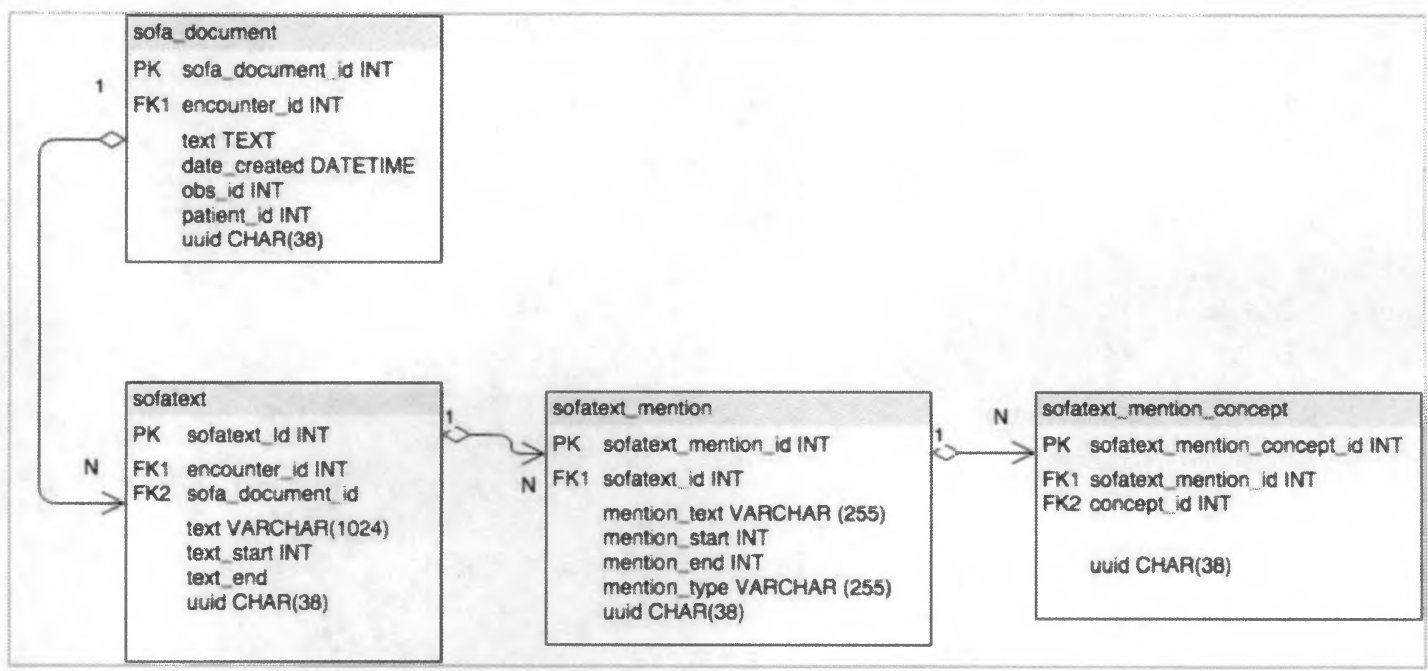

This data model forms a hierarchical structure used to maintain the results of the analysis of a Visit Note. Roughly the tables form this correspondence: 


$$
\begin{array}{ll}
\text { sofa_document } & \Rightarrow \text { Visit Note } \\
\text { sofatext } & \Rightarrow \text { sentence in Visit Note } \\
\text { sofatext_mention } & \Rightarrow \text { entity identified in a sentence } \\
\text { sofatext_mention_concept } & \Rightarrow \text { corresponding OpenMRS Concept for } \\
& \text { the entity, if it exists. Empty otherwise. }
\end{array}
$$

Leveraging the NLPService class and Hibernate ORM mappings (www.hibernate.org), we can read sofa_documents from the database into Java Objects. The corresponding data hierarchy of Java Objects is shown in figure 2.

Figure 2: SofaDocument data hierarchy

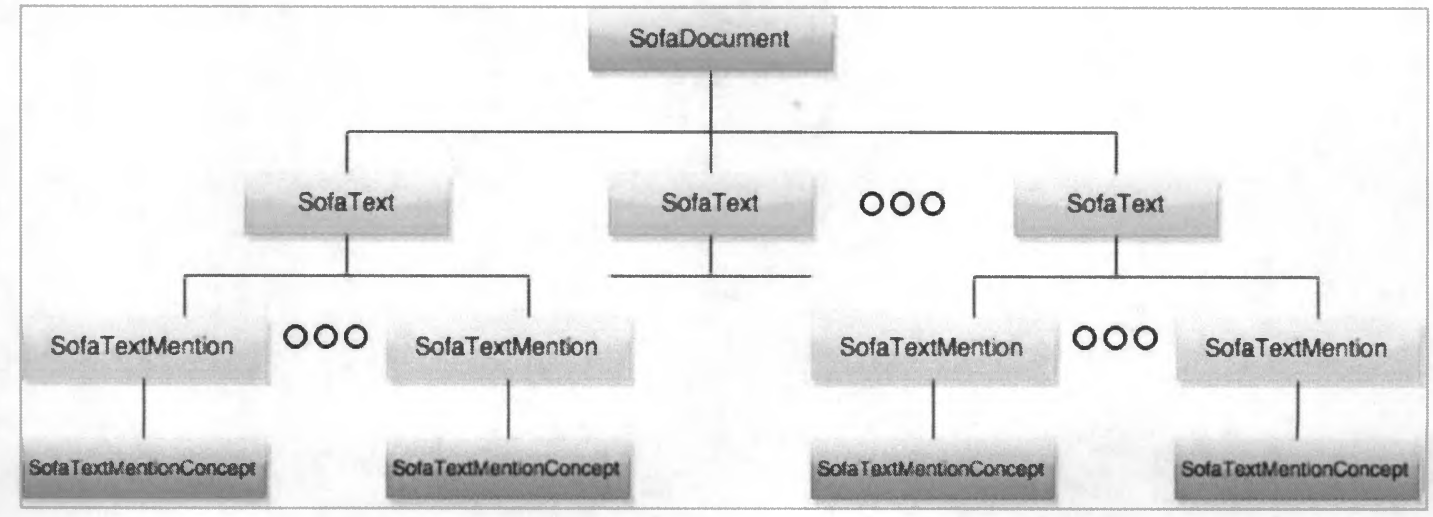

One SofaDocument can contain many SofaText objects. One SofaText can contain many SofaTextMention objects. One SofaTextMention can contain a SofaTextMentionConcept object.

A concrete example of this hierarchy is shown in figure 3 
Figure 3: concrete example of SofaDocument data hierarchy

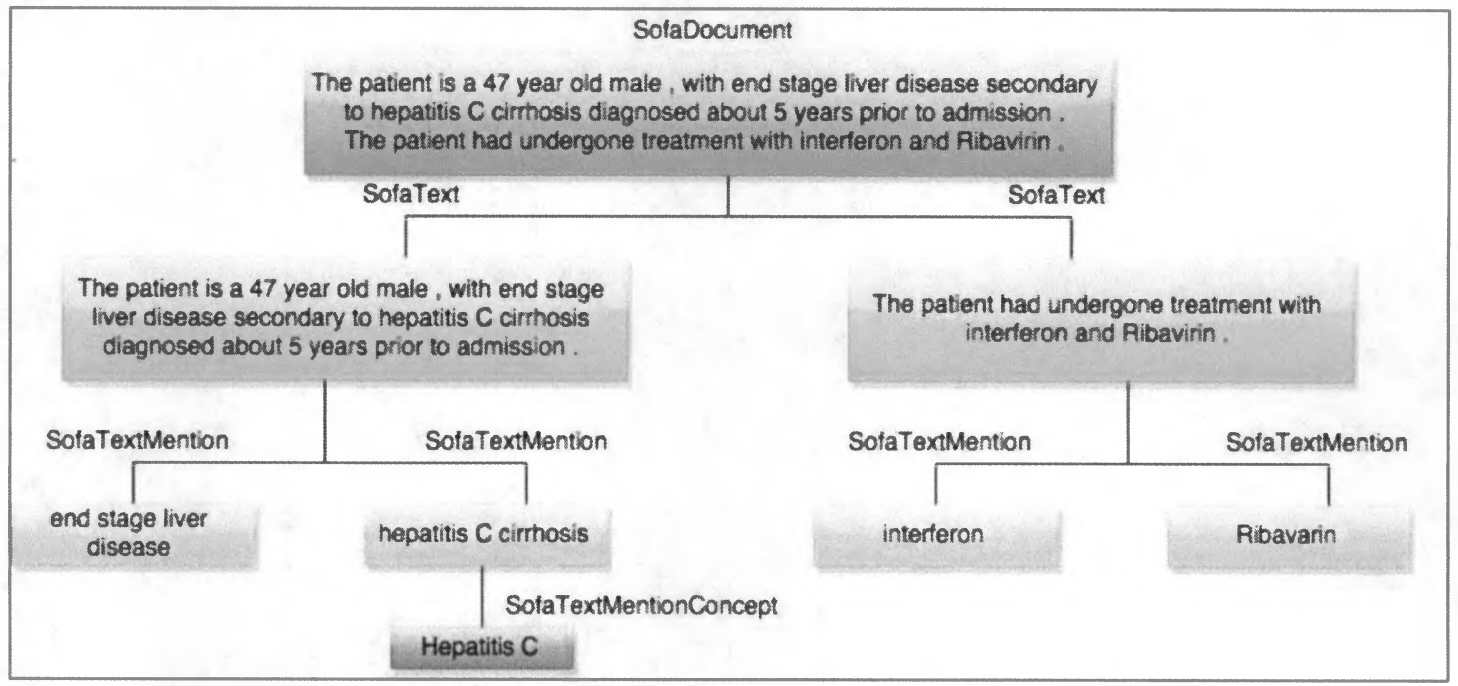

Figure 4: Named Entity Recognition Algorithm

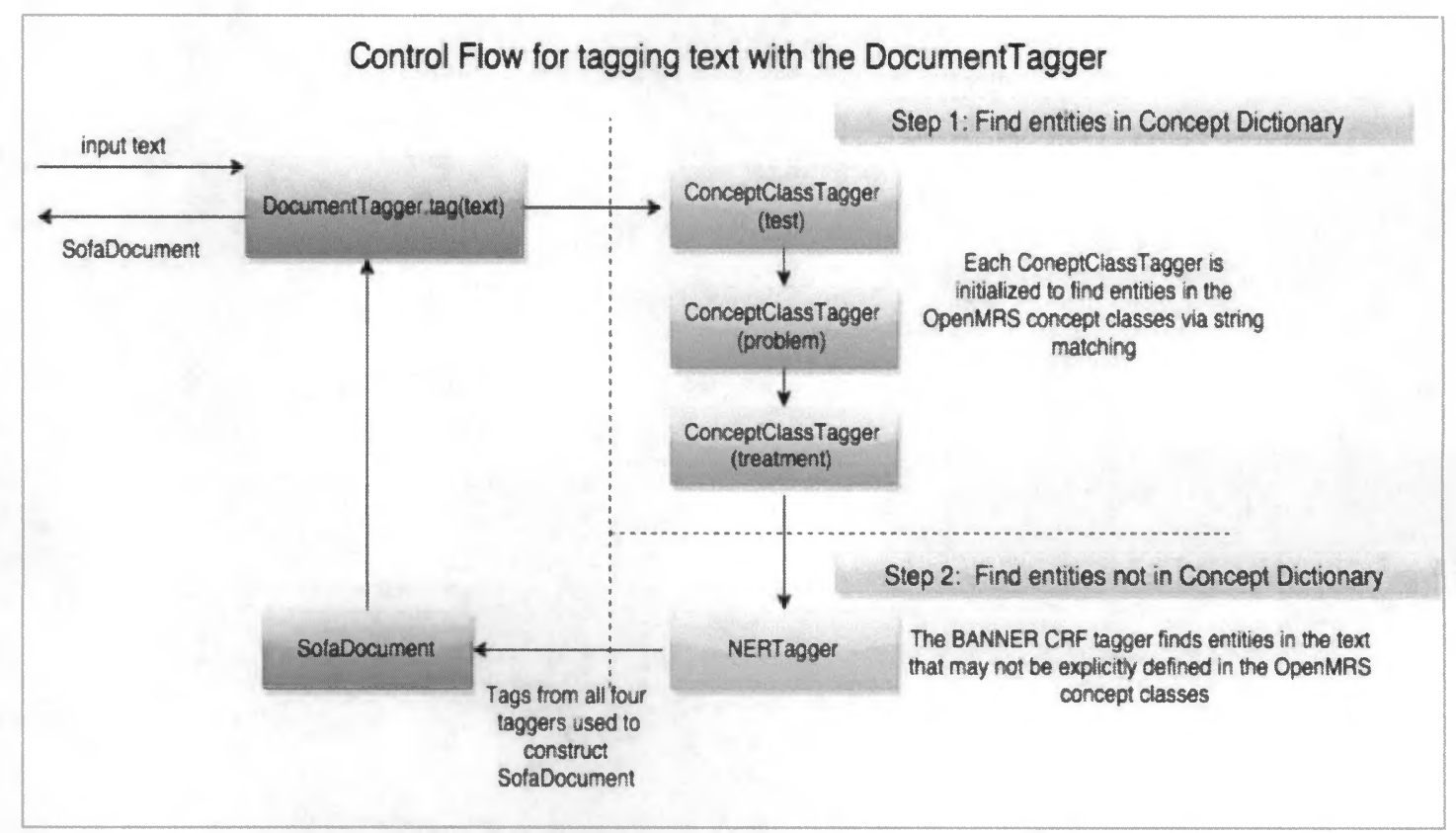

\subsection{Named Entity Recognition Algorithm and Implementation}

The Named Entity Recognition algorithm takes place in two steps. The first step uses the concept class mappings described in section 3.1 to identify concepts and their 
synonyms within the text. The second step uses the machine learning algorithm Conditional Random Fields provided by BANNER (banner.sourceforge.com) to find entities that may not be explicitly noted in the Concept Dictionary. Figure 4 diagrams this process. BANNER was chosen for this module after it showed superior performance when compared with several other open source NER systems.

\subsection{Visit Note Processing with Aspect Oriented Programming} Visit notes are analyzed as they are submitted to the system via Spring's support for Aspect Oriented Programming (https://wiki.openmrs.org/display/docs/OpenMRS+AOP). When a user submits a visit note through the Visit Notes page, the VisitNoteAdvice class interrupts the control flow and processes the text of the Visit Note, saving the results for future presentation. Figure 5 shows this process.

Figure 5: AOP processing of Visit Notes

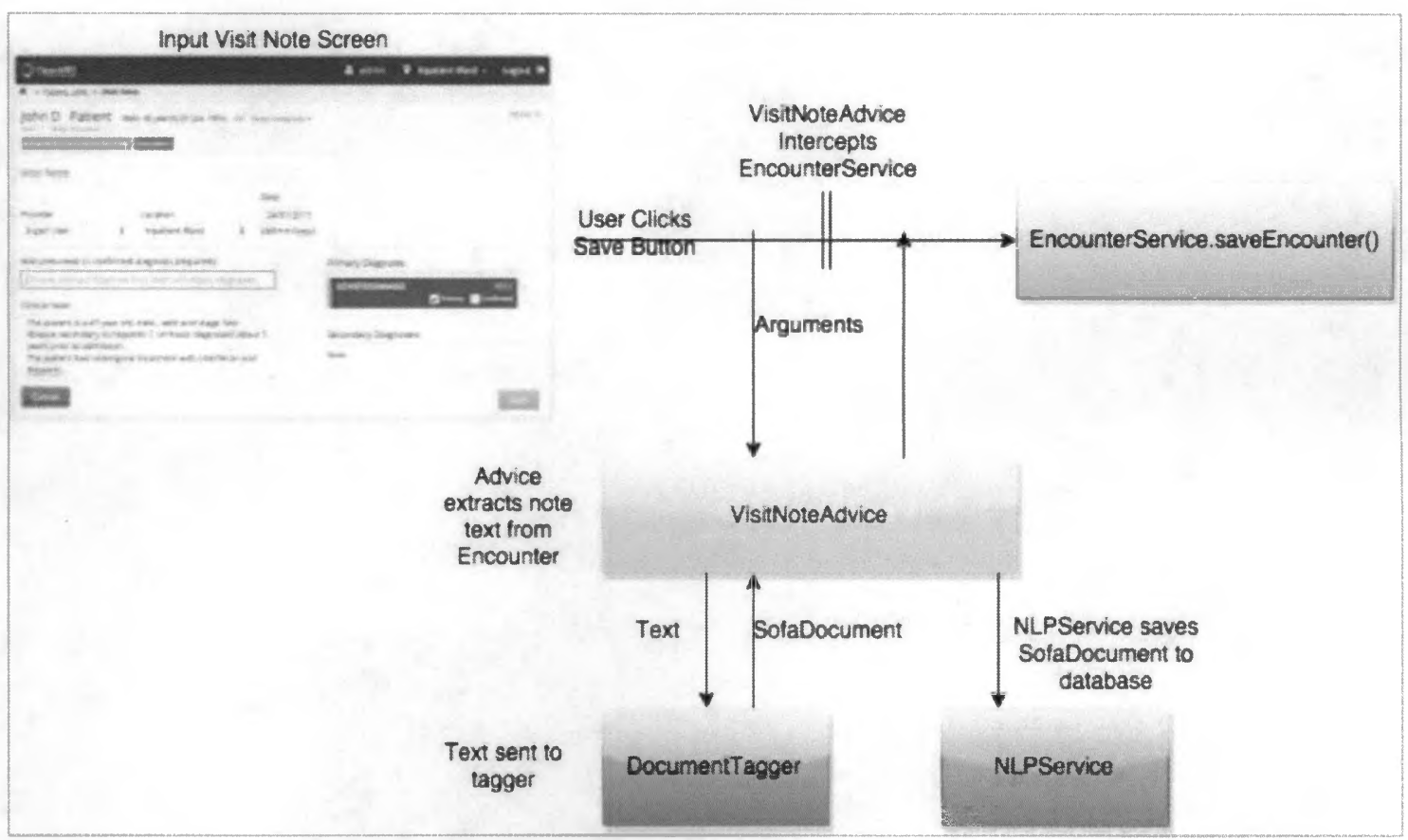


These three components, the data model, the algorithm, and the control flow of capturing a Visit Note make up the foundation of the module.

\section{Application Programming Interface}

There are three main components of the API a developer can use to extend or build on top of this module. Those components are the NLPService class for loading/storing data objects, the SofaDocument and related classes for manipulating the data and the DocumentTagger class for performing NER on strings of text. Below are brief descriptions of each component followed by illustrative code snippets. Finally we provide excerpts from the JavaDocs for the related classes.

NLPService class: This service manages storing and retrieving SofaDocument objects and related data in the database.

SofaDocument, SofaText, SofaTextMention, and SofaTextMentionConcept classes: Objects of these classes are used to manipulate the text data and annotations.

DocumentTagger class: This class provides a simple interface to the developer to execute the NER functionality of the module. 


\subsection{Code Snippets}

The following are three code snippets that illustrate how to use the core API functions.

1. Using the DocumentTagger class to annotate a text string and print out all "problem" entities found in that string:

Figure 6: Tag text with DocumentTagger

\section{Code:}

//text to be analyzed

String text $=$ "The patient is a 47 year old male with "

+ "end stage liver disease secondary to hepatitis C "

+ "cirrhosis diagnosed about 5 years prior to admission";

DocumentTagger tagger $=$ new DocumentTagger () ;

Output:

> end stage liver disease

hepatitis $\mathrm{C}$ cirrhosis 
2. Tag a string of text and save it to the database using the NLPService class.

3. Retrieve all SofaDocuments for patient whose patient_id is 100 , print out the text of the documents.;

Fiqure 7: Tag a string, then save it to the Database using the NLPService

Code:

Patient patient $=$ Context.getPatientService().getPatient(100);

String text $=$ "The patient is a 47 year old male with "

+ "end stage liver disease secondary to hepatitis C "

+ "cirrhosis diagnosed about 5 years prior to admission";

DocumentTagger tagger $=$ new DocumentTagger () ;

SofaDocument sofaDocument $=$ tagger .tagDocument $($ text $)$;

//set the patient associated with the SofaDocument

sofaDocument. setPatient(patient);

//use the NLPService to save the SofaDocument to the DB

Context.getService(NLPService.class). saveSofaDocument(sofaDocument); 
Fiqure 8: Retrieve SofaDocument objects associated with a patient, print the text.

Code:

Patient patient $=$ Context.getPatientService().getPatient(100);

//use NLPService to read all documents for patient from DB

List $<$ SofaDocument $>$ documents $=$ Context.getService(NLPService.class)

-getSofaDocumentsByPatient(patient);

//DocumentTagger iterate over and print all documents

//in this case, we only have 1 document saved

for(SofaDocument $d$ : documents)

System.out.println(d.getText())

Output:

> The patient is a 47 year old male with end stage liver disease secondary to hepatitis $C$ cirrhosis diagnosed about 5 years prior to admission

4. Internally to the DocumentTagger object, code from the BANNER library is being executed to contribute annotations to the text. Here, tagger is an instance of the BANNER CRFTagger class, tokenizer is an instance of the BANNER Tokenizer class, Sentence and Mention are also classes from the BANNER library. Figure 10 gives this example of the CRFTagger tagging a sentence, the result is a list of Mention objects. 
Figure 30: example of BANNER library code

Sentence new_sentence = new Sentence("This is a new sentence containing the problem diabetes");

tokenizer, tokenize (new_sentence);

tagger.tag(new sentence):

\subsection{Javadoc Excerpts}

Note: Many of these classes reference a banner.tagging.Mention object. This object is part of the result of the NER tagging performed by BANNER. It contains information like mention text and mention type that are used in many of the following classes.

\subsubsection{NLPService}

Figure 11: Javadoc excerpts for NLPService

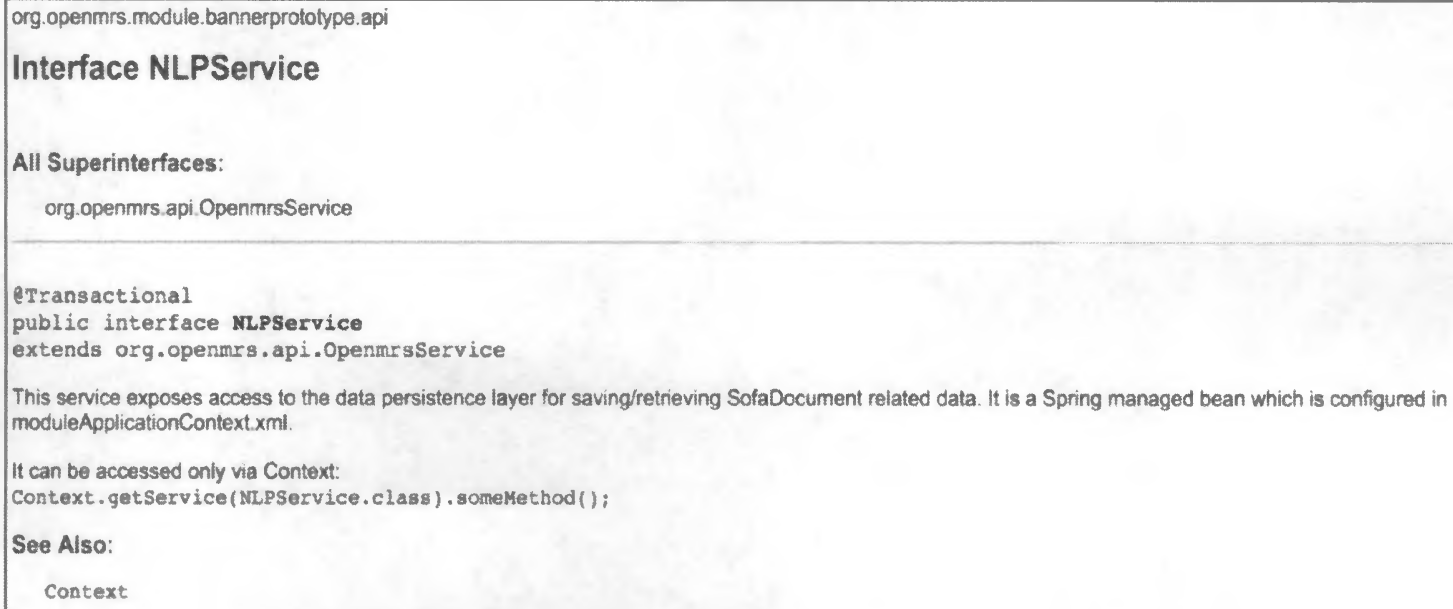




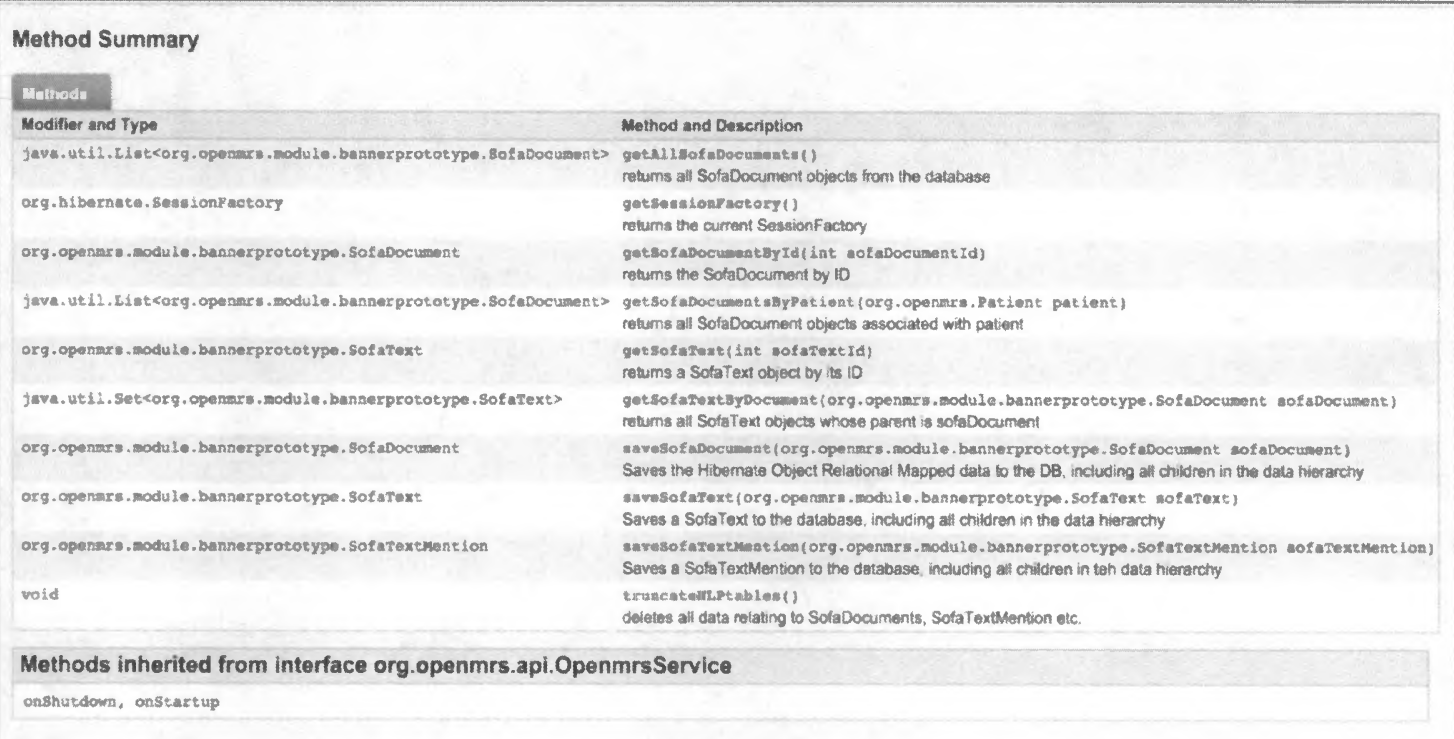

\subsubsection{DocumentTagger}

Figure 12: Javadoc excerpts for DocumentTagger class

org.openmrs.module.bannerprototype.nlp

\section{Class DocumentTagger}

java.lang. Object

org.openmrs.module.bannerprototype.nip.DocumentTagger

All Implemented Interfaces:

java.io.Serializable

public class DocumentTagger

extends java.lang.Object

implements java.io.Serializable

This class exposes the Named Entity Recognition functionality of the module.

This class contains four internal Named Entity Recognizers, three ConceptClass Tagger objects

Each ConceptClass Tagger is initialized to recognize entities in text based on the concept class mappings

set in the Manage Visit Notes Analysis page accessible through the OpenMRS Administration page.

These mappings are stored and read via global properties.

The fourth tagger is an NERTagger object which uses the BANNER CRF library and model specified in the Manager Visit Notes Analysis page 


\section{Method Summary}

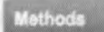

org.openmis. module. bannerprototype.SofaDocument tagDocusent (java.1ang.String document)

This method executes the NER algorithm to find mentions of medical entities within the input text

\section{Methods inherited from class java.lang.Object}

eqquals, getclass, hashcode, notify, notifynil, tostring, wait, wait, wait

\section{Constructor Detail}

\section{DocumentTagger}

public DocunentTagger()

\section{Method Detail}

\section{tagDocument}

public org.openms. module. bannerprototype. Sofabocument tagDocument ( java. lang.String document)

This method executes the NER algorithm to find mentions of medical entities within the input text. Input is a simple String object. Output is a populated SofaDocument including all children in the SofaDocument data hierarchy.

\section{Parameters:}

document - Text to be tagged

Returns:

SofaDocument fully initialized with text and Named Enfities recognized 


\subsubsection{SofaDocument}

SofaDocument objects are created as a result of the DocumentTagger.tag() method. When a visit note is saved by OpenMRS an encounter is generated/saved along with it. Visit Notes are captured and analyzed via AOP when EncounterService.saveEncounter() is called. The encounter associated with this visit note is recorded in the SofaDocument.

Figure 13: Javadoc Excerpts for SofaDocument class

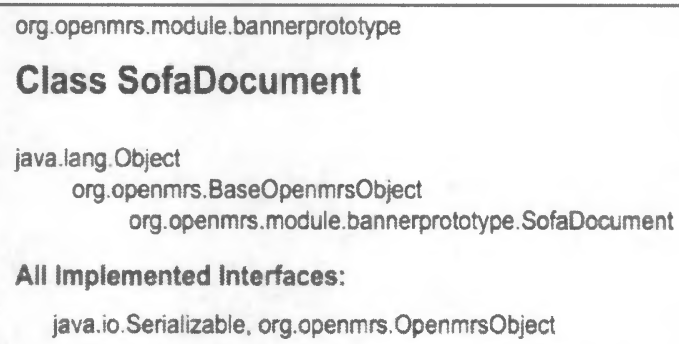

Author:

ryaneshleman

See Also:

Serialized Form

\section{Constructor Summary}

Constructors

Constructor and Description

Sof́abocument ()

Default constructor 


\begin{tabular}{|c|c|}
\hline Methods & \\
\hline Modifier and Type & Method and Description \\
\hline sofarext & $\begin{array}{l}\text { addSofaText ( SofaText sofaText) } \\
\text { add a SofaText object to the set of SofaText objects for this SofaDocument }\end{array}$ \\
\hline java.util.List<BofaTextMeation> & $\begin{array}{l}\text { getalumentions () } \\
\text { Retums all of the SofaTextMention objects found in all the SofaText objects for this } \\
\text { SofaDocument }\end{array}$ \\
\hline java. lang.String & $\begin{array}{l}\text { getAnnotatedima, () } \\
\text { Helper method to generate HTML for controller. }\end{array}$ \\
\hline java.util.Date & getDateCreated() \\
\hline org.openmrs. Encounter & $\begin{array}{l}\text { getBncounter() } \\
\text { Visit Notes are saved via the EncounterService. saveEncounter() method, the } \\
\text { encounter field records the associated encounter. }\end{array}$ \\
\hline java. lang. Integer & $\begin{array}{l}\text { get Id ( ) } \\
\text { This method is required to implement BaseOpenmrsObject, it returns the } \\
\text { sofaDocumentld value }\end{array}$ \\
\hline org.openmrs.Patient & getPatient () \\
\hline java.util.List<sofaTextMention> & $\begin{array}{l}\text { getProblemMentions () } \\
\text { Retums all of the SofaTextMention objects with type 'problem' found in all the SofaText } \\
\text { objects for this SofaDocument }\end{array}$ \\
\hline int & $\begin{array}{l}\text { get Sof aDocuaentId () } \\
\text { sofaDocumentld is a unique identifier for the SofaDocument }\end{array}$ \\
\hline java.util.Set<Sofarext> & getsofarext() \\
\hline java.util.List<SofaTextMention> & $\begin{array}{l}\text { getTestMeations () } \\
\text { Returns all of the SofaTextMention objects with type 'test' found in all the SofaText } \\
\text { objects for this SofaDocument }\end{array}$ \\
\hline java.lang.String & $\begin{array}{l}\text { get Text ( ) } \\
\text { Get the text of the SofaDocument. }\end{array}$ \\
\hline java.util.List<SofarextMention> & $\begin{array}{l}\text { get TreatmeatMentioas () } \\
\text { Returns all of the SofaTextMention objects with type 'treatment' found in all the } \\
\text { SofaText objects for this SofaDocument }\end{array}$ \\
\hline void & setDateCreated(java.util. Date dateCreated) \\
\hline void & setEncounter (org. openmrs. Encounter encounter) \\
\hline void & setId(java.lang.Integer arg0) \\
\hline void & setPatient (org.openmrs.Patient patient) \\
\hline void & setSofaDocumentId(int sofaDocumentId) \\
\hline void & setSofaText (java.util.Set<BofaText> sofaText) \\
\hline void & setrext(java.lang.String text) \\
\hline
\end{tabular}

\section{Methods inherited from class org.openmrs.BaseOpenmrsObject}

equals, geturid, hashcode, setUuid, tostring

\section{Methods inherited from class java.lang.Object}

getclass, notify, notifyAll, wait, wait, wait 


\subsubsection{SofaText}

Figure 14: Javadoc excerpt for SofaText class

org.openmrs. module bannerprototype

\section{Class SofaText}

java.lang. Object

org.openmrs. BaseOpenmrsObject

org.openmrs.module bannerprototype.SofaText

All Implemented Interfaces:

java.io.Serializable, java.lang.Comparable, org.openmrs. OpenmrsObject

public class SofaText

extends org.openmrs.Baseopenmrsobject

implements java.io.Serializable, java.lang.Comparable

This class holds sentence level annotations contained in the SofaDocument

Author:

ryaneshleman

See Also:

Serialized Form

\section{Constructor Summary}

Constructors

Constructor and Description

sotatext ()

Default Constructor

sofarext (java.lang.String text)

sofarext (Java.lang.String text, int textStart, int textEnd) 


\begin{tabular}{|c|c|}
\hline \multicolumn{2}{|l|}{ Methods } \\
\hline Modifier and Type & Method and Description \\
\hline \multirow[t]{2}{*}{ boolean } & addBannerMontion (banner.tagging. Mention m) \\
\hline & $\begin{array}{l}\text { BANNER mentions have a lower priority so use this method to add a mention made by BANNER to a } \\
\text { SofaText, it will not be added if the mention subsumes or is subsumed by a current mention }\end{array}$ \\
\hline \multirow[t]{2}{*}{ void } & $\begin{array}{l}\text { addMentionabdConcepts (banner.tagging. Mention m, } \\
\text { java.util.List<org.openmrs. Concept> concepts) }\end{array}$ \\
\hline & $\begin{array}{l}\text { This method adds a new Mention and associated concepts to a SofaText, there can be overlap between } \\
\text { concept names and multiple concepts can match a string, instead of choosing one concept, we record } \\
\text { them all. }\end{array}$ \\
\hline \multirow[t]{2}{*}{ int } & comparezo (java. lang. Object st) \\
\hline & $\begin{array}{l}\text { retums } 0 \text { if two SofaText objects start at the same index, otherwise returns the distance in characters } \\
\text { between the start index of the two SolaTexts being compared. }\end{array}$ \\
\hline \multirow[t]{2}{*}{ java.lang.string } & getAnnotatedsma( ) \\
\hline & $\begin{array}{l}\text { Helper method to generate HTML, will be moved to controller in future, the html contains the text of the } \\
\text { SofaText object with each mention wrapped in a span tag }\end{array}$ \\
\hline \multirow[t]{2}{*}{ org. openars. Encounter } & getrncounter () \\
\hline & $\begin{array}{l}\text { Visit Notes are saved via the EncounterService saveEncounter() method, the encounter field records the } \\
\text { associated encounter. }\end{array}$ \\
\hline \multirow[t]{2}{*}{ java.lang. Integer } & getId() \\
\hline & returns sofa Textid value, this method is required to implement BaseOpenmrsObject \\
\hline \multirow[t]{2}{*}{ java,util.collection<? extends sofarextmention> } & getProbleass () \\
\hline & retums all SofaTextMention objects with type "problem" in this SofaText object \\
\hline \multirow[t]{2}{*}{ Sofabocument } & getsofabocunent() \\
\hline & retums the parent SofaDocument object for this SofaText \\
\hline \multirow[t]{2}{*}{ int } & getsofaTextId() \\
\hline & retums the unique identifier for this SofaText object \\
\hline \multirow[t]{2}{*}{ java.uti1.Set<SofarextMention> } & getSofarextMention() \\
\hline & $\begin{array}{l}\text { retums the mentions found in this SofaText, there can be overlap between concept names and multiple } \\
\text { concepts match a string, instead of choosing one, we record them all. }\end{array}$ \\
\hline \multirow[t]{2}{*}{ java.util.collection<? extends sofarextMention> } & getresta() \\
\hline & returns all SofaTextMention objects with type "test" in this SofaText object \\
\hline \multirow[t]{2}{*}{ java.lang.String } & getrext() \\
\hline & retums the text string for this SofaText obj \\
\hline \multirow[t]{2}{*}{ int } & getTextEnd() \\
\hline & retums the index into the parent SofaDocument that the SofaTExt ends at \\
\hline \multirow[t]{2}{*}{ int } & getTextstart \{\} \\
\hline & retums the index into the parent SofaDocument that the SofaText begins at \\
\hline \multirow[t]{2}{*}{ java.util.Collection<? extends sofazextrention> } & getrreatments () \\
\hline & retums all SofaTextMention objects with type "treatment" in this SofaText object \\
\hline void & setEncouster (org.openrars. Encounter encounter) \\
\hline void & set Id (java. lang. Integer id) \\
\hline void & setsofaDocument ( Sof aDocument sofabocument) \\
\hline vold & setsofarextId(int sofaTextId) \\
\hline void & setsofarextmention (java.util. Set<sofarextMention> sofaTextMention) \\
\hline void & $\begin{array}{l}\text { setrext ( java. lang. String text) } \\
\text { sets teh text string for this SofaText obj }\end{array}$ \\
\hline void & setTextEnd(int textEnd) \\
\hline void & set Textstart (int textStart) \\
\hline
\end{tabular}




\subsubsection{SofaTextMention}

The NER algorithm executed in DocumentTagger.tag() may find multiple OpenMRS Concepts in a single mention. This is a consequence of overlapping concept names/synonyms in the Concept Dictionary. Instead of forcing the algorithm to choose one Concept, we record them all.

Figure 15: Javadoc excerpt for SofaTextMention class

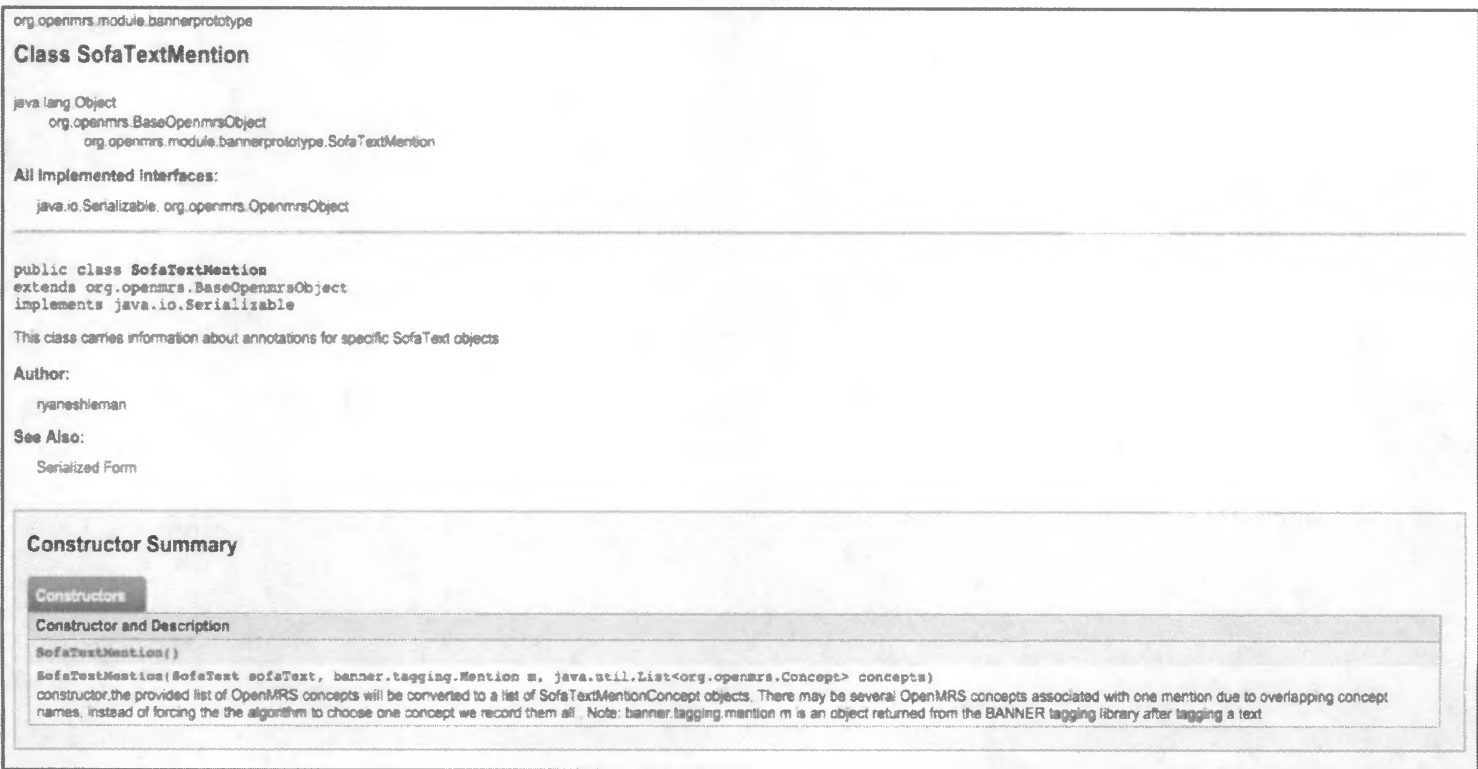




\begin{tabular}{|c|c|}
\hline \multicolumn{2}{|l|}{ Mothods } \\
\hline Modifier and Type & Method and Description \\
\hline \multirow[t]{2}{*}{ vold } & addconcepts ( fava.ut11.List<org.openmrs. Concept> concepts) \\
\hline & $\begin{array}{l}\text { Add list of associated OpenMRS Concept objects associated with this SofaTextMention, these Concepts are identified } \\
\text { during the first String matching phase of the NER algorithm in DocumentTagger.tag() }\end{array}$ \\
\hline \multirow[t]{2}{*}{ java.util.List<org.openms. Concept> } & getConcepts() \\
\hline & $\begin{array}{l}\text { heiper method that returns the OpenMRS Concept objects associated with this SofaTextMention by extracting them } \\
\text { from the SofaTextMentionConcept objects, Concepts are identified during the first String matching phase of the NER } \\
\text { algorithm in DocumentTagger.tag() }\end{array}$ \\
\hline \multirow[t]{2}{*}{ java.lang. Integer } & get Id () \\
\hline & retums sofaTextMentionld value, this method is required to implement BaseOpenmrsObject \\
\hline \multirow[t]{2}{*}{ int } & getwantionBad () \\
\hline & returns the index into the SofaTExt where the mentions ends \\
\hline \multirow[t]{2}{*}{ int } & getMentionstart () \\
\hline & retums the index into the SofaText where the mention starts index of start of mention within SofaText \\
\hline \multirow[t]{2}{*}{ java.lang.String } & getMentionText() \\
\hline & return the text string associated with this mention, for example 'cirthosis' \\
\hline \multirow[t]{2}{*}{ java.lang.String } & getMeationType() \\
\hline & returns the mention type as a string, for example "problem" \\
\hline \multirow[t]{2}{*}{ sofarext } & get Sofarext() \\
\hline & retums the parent SofaText for this object \\
\hline \multirow[t]{2}{*}{ java.util.Set<SofaTextilentionConcept> } & getsofaTextMentloaconcept () \\
\hline & $\begin{array}{l}\text { returns the children SofaTextMentionConcept objects associated with the SofaTextMention object, it may be an empty } \\
\text { set. }\end{array}$ \\
\hline \multirow[t]{2}{*}{ int } & getsofaTextmeationid() \\
\hline & unique identifier for this SolaTextMention object \\
\hline void & setId (java.lang. Integer id) \\
\hline void & setMeationend (int mentionsnd) \\
\hline vold & $\begin{array}{l}\text { wotMentionstart (int mentionstart) } \\
\text { set start index of mention }\end{array}$ \\
\hline void & aetreationtext (java.lang.String aentionText) \\
\hline void & setNentiontype(java. lang.String mentiontype) \\
\hline void & wetSofaText (SofaText sofaText) \\
\hline void & set \&ofaTextMentionConcept (java.util. Set<SofaTextMentionConcept) sofaTextMentionConcept) \\
\hline void & setsofarextMentionId(int sofaTextMentionid) \\
\hline
\end{tabular}




\subsubsection{SofaTextMentionConcept}

Figure 16: Javadoc excerpt for SofaTextMentionConcept class

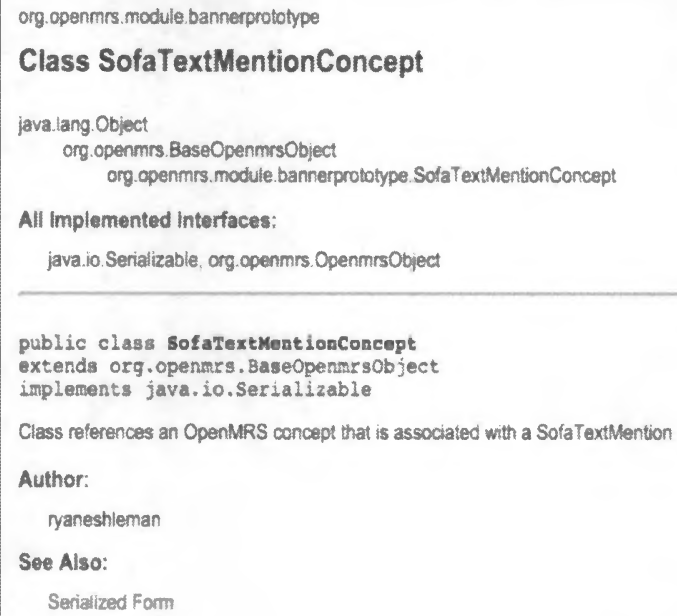




\section{Method Summary}

\section{Welhods}

Modlfier and Type

org. openmrs. Concept

Method and Description

int

getcoacept ()

retums the OpenMRS concept associated with this object

getConceptrd ()

java.lang. String

retums the id associated with the OpenMRS Concept contained in this object

getconcoptwame(1)

get the name of the concept. for example "tuberculosis"

java. lang. Integer

get Id ()

retums sofeTExtMentionConceptld value, required to implement Base OpenmrsObject

SofaTextllention

getsofatextment (os ()

get the parent Sola TextMention

Int

getsofarextluentionconceptld()

unique identifier for this object

set Concept forq. openars. Concept concept

void

setConceptid (int conceptId)

setCoaceptirane ( Java.lang.String conceptName)

set the concept Name

cet Id ( java. Iang. Integer argo)

setsofarertMention (SofaTextMontion sofaTextMention)

setSofaTextMentionCoaceptId (int sofaTextMentionConceptId)

void

Methods inherited from class org.openmrs.BaseOpenmrsObject

equals, getUuid, hashcode, setuuid, tostring

Methods inherited from class java.lang.Object

getclass, notify, notifyAl, wait, wait, wait 


\section{Package Structure}

Module package structure, some common directories and folders are omitted. For example the location of .js/css files and pages/fragments.

Figure 35: Module API package structure

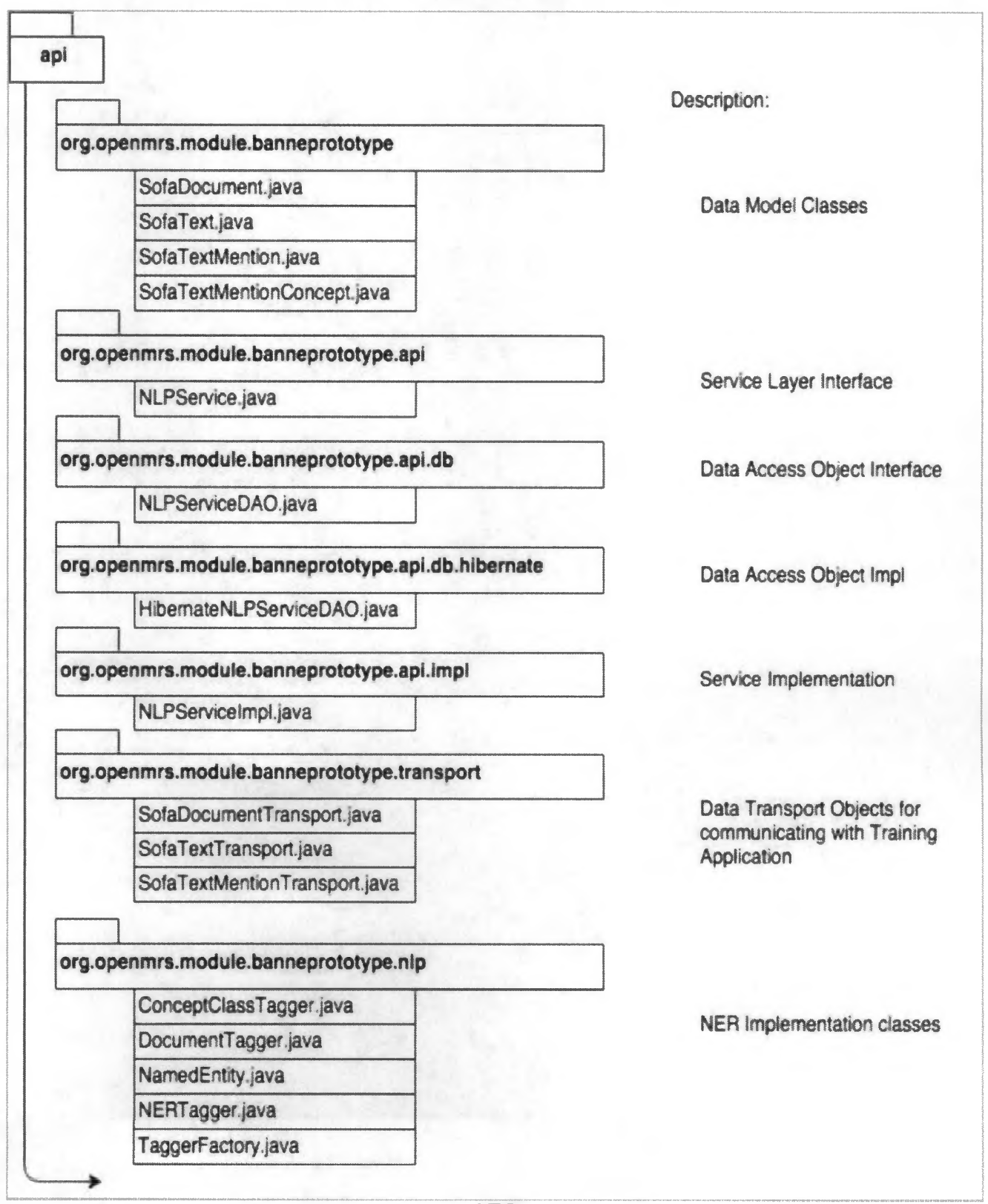


Figure 36: Module Omod package structure

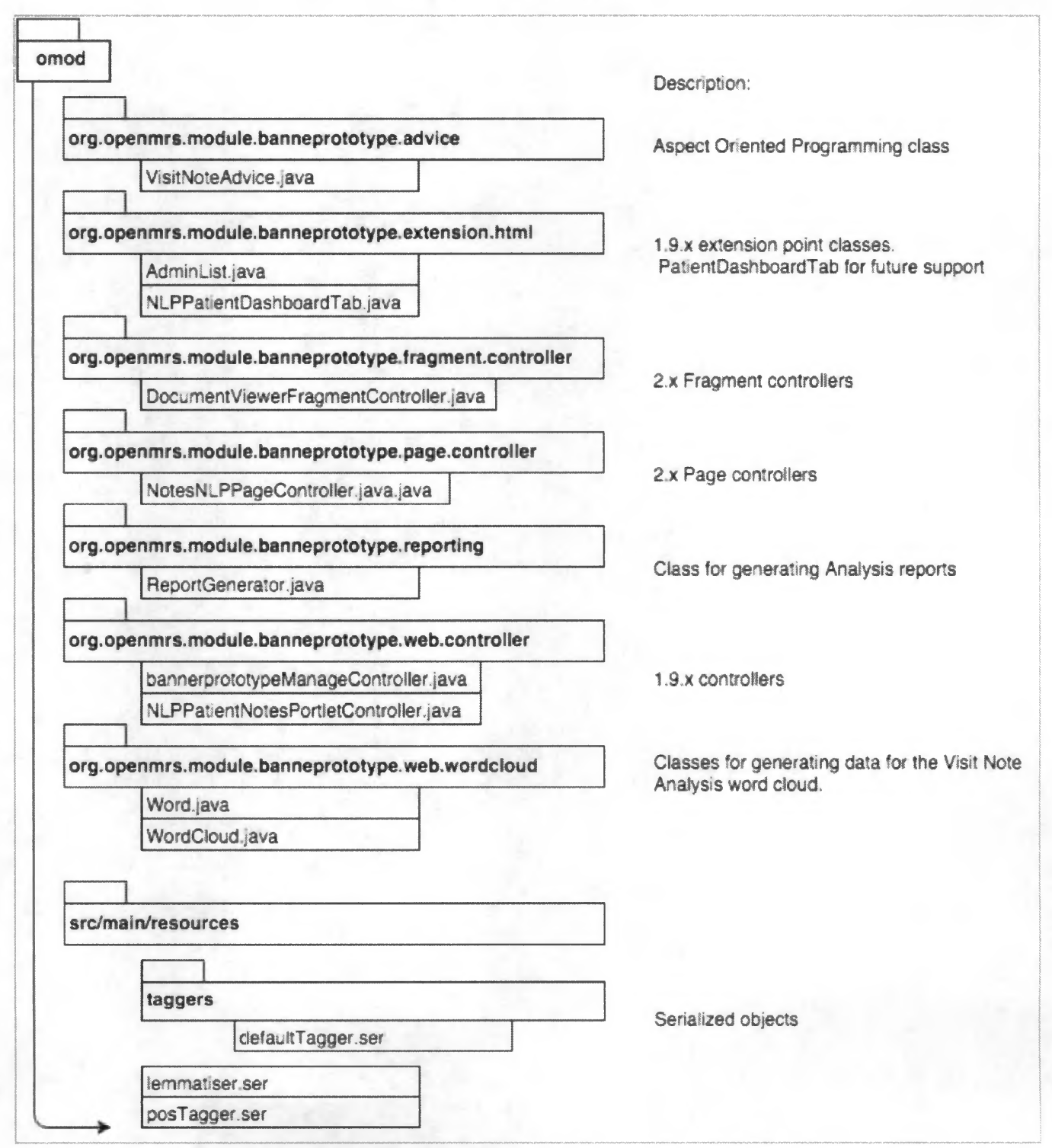


Appendix 4:

Review of Graphical Models for Named Entity Recognition 


\section{A Review of Graphical Models for Named Entity Recognition}

In this appendix, we will provide a brief overview of some of the graphical modelling techniques used in Named Entity Recognition (NER) and Natural Language Processing to model text as sequential data. Text is often modeled sequentially because of the observation that the order in which a word appears in a sentence has a strong effect on the meaning and function of the word within the sentence. This information is often lost with other text processing approaches, such as Bag-of-Words[11] where sequence is completely thrown out.

In general, the sequential model for NER takes the text sequence as input, and attempts to assign "states" to each word in the sequence. For example, given a two state model where they two possible states are "problem" and "other." The state assignments for the sentence "The patient was diagnosed with Hepatitis C" would be The ${ }^{\text {other }}$ patient ${ }^{\text {other }}$ was $^{\text {other }}$ diagnosed ${ }^{\text {other }}$ with $^{\text {other }}$ Hepatitis ${ }^{\text {problem }} C^{\text {problem }}$. The models discussed in this appendix are, in essence, different models designed to arrive at the proper state sequence, given an input text. The document presents the main ideas behind the different models, and we would refer the reader to the references for a more in depth discussion of these topics. 
The rest of this appendix is structured as follows. Section 1 provides an introduction to Markov Chains, which is a fundamental component of the subsequent models. The following sections build on each other to present more and more sophisticated models. Section 2 presents Hidden Markov Models which extends the Markov Chain by adding the notion of hidden states. Section 3 describes Maximum Entropy Markov Models, which expand the feature set that the model can employ. Finally, Section 4 introduces Conditional Random Fields which addresses some problems inherent in the previous models.

\section{Introduction and background on Markov Chains}

Many machine learning algorithms and statistical approaches have been developed with the intention of assigning a class to a set of input data (for example Random Forests [1], Support Vector Machines[2], and Neural Networks [3]) or predicting values given an input of observations. For example, a regression model [4] may be used to predict the value of a house given a number of characteristics about that house, such as square footage and number of rooms. These are certainly important tasks with a wide range of applications. This approach to data analysis does make a very strong assumption about how the data is generated. Classifiers assume that the sequence in which the data was generated has no effect on the classification. As we described earlier, this sequence information often plays a very important role in NER and NLP and the sequence agnostic assumption of traditional classification algorithms often makes them inadequate for the task. 
Traditional classification leaves open the whole domain of real world systems that produce output in the form of a signal, or sequence of observations. Underlying properties of these signals can vary. A signal can be discrete in nature as in letters from an alphabet and parts of speech. A signal can also be continuous, as in temperature measurements and audio samples. Observations made about a signal are highly dependent on the sequence in which the observation was made. For example, the toy observation sequence $[1,-1,1,-1]$ is much different than $[1,1,-1,-1]$

This sequentially dependent data requires a different kind of model than our standard classifiers in order to properly characterize the data. There are generally two types of models that can be used for these kinds of signals, deterministic and statistical models. The deterministic models take known information about the signal, for example that it is a sinusoid, or the sum of exponentials, and only require that some parameters be estimated, such as amplitude and frequency. The second category of sequential model is the statistical model, which assumes that the signal can be characterized by a parametric random process and that the parameters can be accurately estimated in a principled way. A Markov process [5] is an example of a statistical process.

In the fields of research relating to text and language processing, the use of Markov Processes and their derivatives have been found to be very successful in practice. A Markov process, or Markov Chain is a probabilistic finite state machine with transition probabilities associated with transitions from $\mathrm{S}_{\mathrm{i}}$ to $\mathrm{S}_{\mathrm{j}}$. Formally, a Markov Chain is defined by $N$ states $S_{1}, S_{2}, \ldots S_{n}$, and $N x N$ transition probabilities $a_{i j}=P\left(q_{t}=S_{j} \mid q_{t-1}=S_{i}\right)$ where $q_{t}$ is 
the state of the machine at time $t$. In words, $a_{i j}$ is the probability that the machine in state $\mathrm{S}_{\mathrm{i}}$ will transition to state $\mathrm{S}_{\mathrm{j}}$. A simple example of a Markov chain is given below and is a Model for transition probabilities between states of the weather.

Figure 4: Markov Chain

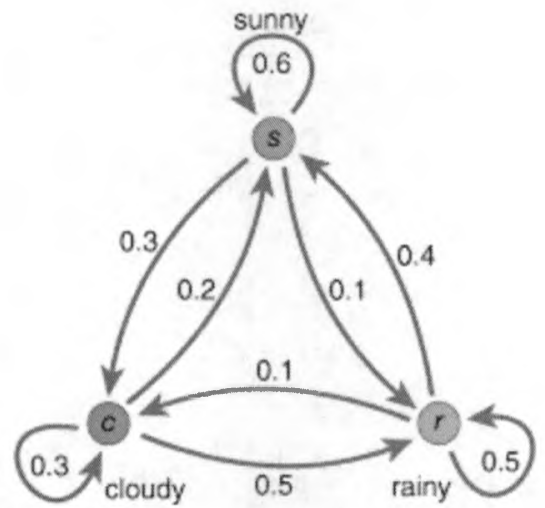

Source: http://bit-player.org/wp-content/extras/markov/art/weather-model.png

Here, the three states of the model are:

1. sunny

2. cloudy

3. rainy

And the transition probabilities $\mathrm{a}_{\mathrm{ij}}$ can be easily represented as a transition matrix $\mathrm{A}$ as follows:

$$
\mathrm{A}=\left\{\mathrm{a}_{\mathrm{ij}}\right\}=\left[\begin{array}{lll}
.6 & .3 & .1 \\
.2 & .3 & .5 \\
.4 & .1 & .5
\end{array}\right]
$$

Where $A[1][1]$ is the probability that a machine in state 1 , sunny, will transition to state 1,sunny, which is .6. Using this weather model, we can compute the probability of the 
weather pattern sunny-sunny-cloudy-rainy-sunny (assuming the first state is sunny, or, $\mathrm{P}\left(\mathrm{q}_{1}\right.$ $=$ Sunny $)=1)$. This corresponds to the sequence $O=\left\{S_{1}, S_{1}, S_{2}, S_{3}, S_{1}\right\}$. So, the probability of the observation sequence $\mathrm{O}$, given our markov model of the weather is:

$$
\begin{aligned}
\mathrm{P}(\mathrm{O} \mid \text { model }) & =\mathrm{P}\left(\mathrm{S}_{1} \mid \mathrm{S}_{1}\right) \mathrm{P}\left(\mathrm{S}_{2} \mid \mathrm{S}_{1}\right) \mathrm{P}\left(\mathrm{S}_{3} \mid \mathrm{S}_{2}\right) \mathrm{P}\left(\mathrm{S}_{1} \mid \mathrm{S}_{3}\right) \\
& =\mathrm{a}_{11} * \mathrm{a}_{12} * \mathrm{a}_{23} * \mathrm{a}_{31} \\
& =.6 * .3 * .5 * .4 \\
& =.036
\end{aligned}
$$

To fully specify our model and get rid of our start state assumption, we need to describe start state probabilities $\pi_{\mathrm{i}}=\mathrm{P}\left(\mathrm{q}_{1}=\mathrm{S}_{\mathrm{j}}\right)$. In our weather model, $\pi_{1}=1$ and $\pi_{2}=\pi_{3}=$ 0 . But these numbers could be any values between 0 and 1 so long as the sum of the $\pi$ values satisfies the stochastic constraint of summing to 1 .

Markov chains allow us to ask a number of interesting questions, for example, one can compute the probability that the model will remain in state $\mathrm{S}_{1}$ for exactly $\mathrm{N}$ number of days. This corresponds to the observation sequence $\mathrm{O}=\left\{\mathrm{S}_{1}, \mathrm{~S}_{1}, \ldots, \mathrm{S}_{1}, \mathrm{~S}_{\mathrm{i}} \neq \mathrm{S}_{1}, \mathrm{i}=\mathrm{N}+1\right\}$. That is $\mathrm{N}$ instances of $\mathrm{S}_{1}$ and then a transition out of $\mathrm{S}_{1}$.

The ability to ask and answer complex questions given Markov Processes has lead to an interest in developing new models on top of it to ask even more complex questions relating to among other things, Text Processing and Information Extraction.

In the following sections, we will review three such models, Hidden Markov Models, Maximum Entropy Markov Models, and Conditional Random Fields. 


\section{Hidden Markov Models}

Markov chains are powerful and allow us to model sequential observations, as described above. However, with these models, we assume that the observations correspond directly to the state. For example, our state was sunny, so our observation was sunny. But there are some situations where the state itself is not directly observable, so the observations that we make are not the states of the model. The state can only be observed through some probabilistic process that is a function of the state itself. In this case, the state is hidden and we now have to expand our model to fit these new restrictions. The result is what we call a Hidden Markov Model [6].

A classic example to illustrate HMMs is "The Urn and the Ball" example. In the Urn and the Ball, we assume that we have $\mathrm{N}$ glass urns in a room and that each urn is filled with a large number of colored balls. Each ball can be 1 of $M$ unique colors. The distribution of the color of balls in each urn is known and independent of the distributions of balls in the other urns. We make observations in the following way, a genie picks an urn according to an initial urn distribution and chooses a ball from the urn according to the distribution of balls in the chosen urn. The color of this ball is the first observation. The genie replaces the ball to its originating urn, then, through some random process, the genie chooses the next urn and grabs another ball. This is the second observation. This process 
is repeated and the observations are collected. At each time t of the sequence, we are never explicitly told which urn the genie is extracting the ball from. At the end of the process, we have a finite sequence of colors. Given this finite color sequence, we can use an HMM to estimate the sequence of urns, or states, that the colors were extracted from.

A Hidden Markov Model has five components. The number of states N, the number of observations $\mathrm{M}$, the state transition probabilities $\mathrm{A}$, the per state observation probabilities $\mathrm{B}$, and the initial state distributions $\pi$. Formally, an HMM is defined as:

$$
\begin{aligned}
& \mathrm{HMM}=\{\mathrm{N}, \mathrm{M}, \mathrm{A}, \mathrm{B}, \pi\} \\
& \mathrm{N}=\text { number of states } \\
& \mathrm{M}=\text { number of observations } \\
& \text { A } \quad=\left\{\mathrm{a}_{\mathrm{ij}}, \ldots\right\} \quad \text { where } \mathrm{a}_{\mathrm{ij}}=\mathrm{P}\left(\mathrm{q}_{\mathrm{t}+1}=\mathrm{S}_{\mathrm{j}} \mid \mathrm{q}_{\mathrm{t}}=\mathrm{S}_{\mathrm{i}}\right) \\
& \mathrm{B} \quad=\left\{\mathrm{b}_{\mathrm{j}}(\mathrm{k})\right\} \quad \text { where } \mathrm{b}_{\mathrm{j}}(\mathrm{k})=\mathrm{P}\left(\mathrm{K} \text { at } \mathrm{t} \mid \mathrm{q}_{\mathrm{t}}=\mathrm{S}_{\mathrm{j}}\right) \\
& \text { And } \mathrm{K} \text { is an observation } 1 \leq \mathrm{k} \leq \mathrm{M} \\
& \pi \quad=\left\{\pi_{\mathrm{i}}\right\} \quad \text { where } \pi_{\mathrm{i}}=\mathrm{P}\left(\mathrm{q}_{1}=\mathrm{S}_{\mathrm{i}}\right)
\end{aligned}
$$

It is often simplified to:

$$
\lambda=\{\mathrm{A}, \mathrm{B}, \boldsymbol{\pi}\}
$$

In relating this to our Urn and Ball example, we would say that $\mathrm{N}$ is the number of Urns, $\mathrm{M}$ is the number of colors that a ball can take, A corresponds to the transition probabilities the genie uses to pick a new state $\mathrm{q}_{\mathrm{t}+1}=\mathrm{S}_{\mathrm{j}}$ from state $\mathrm{S}_{\mathrm{i}}$. B is the distribution of ball/colors in each urn and $\pi$ is the probability for each state that the genie will pick it first. 
To generate an observation sequence, given a specified HMM, we take the following steps. 1. Choose the initial state $\mathrm{q}_{\mathrm{t}}=\mathrm{S}_{\mathrm{i}}$ where $\mathrm{t}=1$, based on the initial distributions in $\pi .2$. Generate an observation using the per-state distributions given in $b_{i}(k)$. 3. Transition to a new state $\mathrm{q}_{t+1}=\mathrm{S}_{\mathrm{j}}$ based on the transition probabilities given in A. 4. Increment $t=t+1$. Repeat steps 2-4 until desired number of observations are generated.

This leads us to the 'Three Basic Problems of HMMs. These problems are solved in order to make real world applications of HMMs possible. The problems are the following: 1. Given an observation sequence $\mathrm{O}$ and an HMM $\lambda$, how do you compute $\mathrm{P}(\mathrm{O} \mid \lambda)$. 2. Given an observation sequence $\mathrm{O}$ and $\operatorname{HMM} \lambda$, how do we find the state sequence $\mathrm{Q}=\left\{\mathrm{q}_{1}, \mathrm{q}_{2}, \ldots, \mathrm{q}_{\mathrm{n}}\right\}$ that is 'most optimal.' 'Most optimal' also needs to be defined. This step can be considered the inference step, where a trained model is used to find the most likely sequence of states, or tags, for a set of observations. In an NER application, states would be tags, and observations would be words in a sentence. 3 . Possibly the most important problem, given a sequence of observations how do we learn the HMM parameters in order to maximize $\mathrm{P}(\mathrm{O} \mid \lambda)$.

To address problem 1: find $\mathrm{P}(\mathrm{O} \mid \lambda)$, One can come up with a simple naïve approach of enumerating all possible state sequences and summing $\mathrm{P}\left(\mathrm{O} \mid \mathrm{Q}_{\mathrm{i}}, \lambda\right) \mathrm{P}\left(\mathrm{Q}_{\mathrm{i}} \mid \lambda\right)$ for each sequence $\mathrm{Q}_{\mathrm{i}}$. We can break the computation down into its components. Given a state sequence, one can easily compute $\mathrm{P}(\mathrm{Q} \mid \lambda)$ by the method given above for regular Markov Models. To find $\mathrm{P}(\mathrm{O} \mid \mathrm{Q}, \lambda)$ we compute the product of $\mathrm{b}_{\mathrm{i}}\left(\mathrm{O}_{\mathrm{i}}\right)$ for every state in $\mathrm{Q}$. Any student of algorithms will look at this brute force approach and see that its time complexity 
of $\mathrm{O}\left(2 \mathrm{tn}^{\mathrm{t}}\right)$ is infeasible and quickly becomes intractable for even moderate values of $\mathrm{n}$ and t.

Fortunately, there exists a dynamic programming approach that reduces the complexity from $\mathrm{O}\left(2 \mathrm{tn}^{\mathrm{t}}\right)$ to $\mathrm{O}\left(\mathrm{tn}^{2}\right)$. Which, given $\mathrm{N}=5$, and $\mathrm{T}=100$, reduces the number of computations from $\sim 10^{72}$ to $\sim 2500$ ! This approach is called the Forward-Backward Procedure and is defined by the recurrence relation:

Forward Variable $\alpha$ defined as:

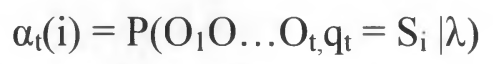

Forward Variable initialized as:

$$
\alpha_{1}(\mathrm{i})=\pi_{\mathrm{i}} \mathrm{b}_{\mathrm{i}}\left(\mathrm{O}_{1}\right), \quad 1 \leq \mathrm{i} \leq \mathrm{N}
$$

Recurrence step:

$$
\begin{array}{r}
\alpha_{\mathrm{t}+1}(\mathrm{i})=\left[\sum_{i=1}^{N} \alpha_{\mathrm{i}}(\mathrm{i}) \mathrm{a}_{\mathrm{ij}}\right] \mathrm{b}_{\mathrm{j}}\left(\mathrm{O}_{\mathrm{t}+1}\right) 1 \leq \mathrm{t} \leq \mathrm{T}-1 \\
\quad 1 \leq \mathrm{j} \leq \mathrm{N}
\end{array}
$$

Finally:

$$
\mathrm{P}(\mathrm{O} \mid \lambda)=\sum_{i=1}^{N} \alpha_{\mathrm{T}}(\mathrm{i})
$$

Using the recurrence, we effectively build an $\mathrm{N} x \mathrm{~T}$ grid corresponding to the values of the forward variable computed from time $\mathrm{t}=1$ to time $\mathrm{t}=\mathrm{T}-1$ for all states. Each index in the grid corresponds to the probability of arriving at state $\mathrm{N}$ at time $\mathrm{t}$. Once the grid has been constructed up to time $\mathrm{T}-1$, we can find the final value by summing up the probabilities of 
arriving at $\mathrm{O}_{t}$ from all $\mathrm{N}$ states at time $\mathrm{T}-1$. This gives us the final probability of our observation sequence $\mathrm{O}$ given $\lambda$.

The second of the three basic HMM problems is regarding how to find an 'optimal' state sequence given an observation sequence $\mathrm{O}$ and an $\operatorname{HMM} \lambda$. The first sub question to answer is, how do we define an optimal sequence. Two optimality criteria come to mind. We can optimize for the largest number of individually correct states or for the most likely complete sequence of states. To optimize for the largest number of correct states, we need to introduce two new definitions. The Backward Variable $\beta$ is a corollary to the Forward Variable and is defined as:

$$
\beta_{\mathrm{t}}(\mathrm{i})=\mathrm{P}\left(\mathrm{O}_{\mathrm{t}} \mathrm{O}_{\mathrm{t}+1} \ldots \mathrm{O}_{\mathrm{T}} \mid \mathrm{q}_{\mathrm{t}}=\mathrm{S}_{\mathrm{i}}, \lambda\right)
$$

Where the Forward Variable was used to construct a probability grid from time $t=1$ to $t=$ T, one can think of the Backward Variable as being used to construct this probability grid in the reverse direction, from $t=T$ to $t=1$. To tie these two variables together we can observe that:

$$
\mathrm{P}(\mathrm{O} \mid \lambda)=\sum_{i=1}^{N} \alpha_{\mathrm{t}}(\mathrm{i}) \beta_{\mathrm{t}}(\mathrm{i}) \quad 1 \leq \mathrm{t} \leq \mathrm{T}-1
$$

The second variable to define is the probability of being in state $\mathrm{i}$ at time $\mathrm{t}$ :

$$
\gamma_{\mathrm{t}}(\mathrm{i})=\mathrm{P}\left(\mathrm{q}_{\mathrm{t}}=\mathrm{S}_{\mathrm{i}} \mid \mathrm{O}, \lambda\right)
$$

Accordingly, we can compute $\gamma_{\mathrm{t}}(\mathrm{i})$ as the following: 


$$
\gamma_{\mathrm{t}}(\mathrm{i})=\frac{\alpha_{t}(i) \beta_{t}(i)}{P(O \mid \lambda)}
$$

Now we can use $\gamma_{\mathrm{t}}(\mathrm{i})$ to find the sequence of states that maximizes the number of correct states as simply:

$$
\mathrm{q}_{\mathrm{t}}=\operatorname{argmax}\left(\gamma_{\mathrm{t}}(\mathrm{i})\right) \quad 1 \leq \mathrm{t} \leq \mathrm{T}-1
$$

This optimization criterion and method may be usefully in some situations, but most commonly, the desired outcome is to find the most likely continuous state sequence for a given $\mathrm{O}$ and $\lambda$. In other words, to optimize $\mathrm{P}(\mathrm{S} \mid \mathrm{O}, \lambda)$. As with the solution to problem 1 , the naïve approach would be to enumerate all possible state sequences, which has an intractably large number of computations. And again, as with problem 1, there is a dynamic programming approach to solve it. The algorithm is called the Viterbi Algorithm and bears a strikingly resemblance to the Forward-Backward procedure. The main difference is that in the FB procedure, $\alpha_{t+1}(i)$ is defined as the sum of the transition probabilities from time $\alpha_{t}$ to $q_{t+1}$, in the Viterbi algorithm, we use the maximum value of the transition from $t$ to $\mathrm{t}+1$ and $\mathrm{q}_{\mathrm{t}+1}$. Or, a little more formally, we replace the forward variable, $\alpha$, with a new variable

$$
\begin{aligned}
& \delta_{\mathrm{t}}(\mathrm{i})=\max \mathrm{P}\left(\mathrm{q}_{1} \mathrm{q}_{2} \ldots \mathrm{q}_{\mathrm{t}=\mathrm{I}}, \mathrm{O}_{1} \mathrm{O}_{2} \ldots \mathrm{O}_{\mathrm{t}} \mid \lambda\right) \\
& \delta_{\mathrm{t}+1}(\mathrm{i})=\left[\max \delta_{\mathrm{t}}(\mathrm{i}) \mathrm{a}_{\mathrm{ij}}\right] * \mathrm{~b}_{\mathrm{j}}\left(\mathrm{O}_{\mathrm{t}+1}\right)
\end{aligned}
$$

Using this variable, we can construct an $\mathrm{N} \mathrm{x} \mathrm{T} \mathrm{grid,} \mathrm{as} \mathrm{in} \mathrm{the} \mathrm{FB} \mathrm{procedure,} \mathrm{from} \mathrm{time} \mathrm{t}=$ 1 to $\mathrm{t}=\mathrm{T}$. This will give us the maximum probability of a state sequence, and if we keep track of the transitions that gave us max values at each step, we can use a backtracking 
procedure in order to retrace the algorithms steps and determine the most likely state sequence.

Problem 3, which can be viewed as learning the model parameters given an observation sequence, is the most involved of the 3 problems. Unfortunately, no analytic approach has yet been discovered to solve this problem and the values must be estimated. Moreover, the estimation procedure, based on the EM algorithm[7], produces locally optimum parameter values. To begin, we define $\xi_{t}(i, j)$ as the probability of transitioning from $S_{i}$ to $S_{j}$ from $t$ to $t+1$. Or:

$$
\xi_{t}(i, j)=P\left(q_{t}=S_{i}, q_{t+1}=S_{j} \mid O, \lambda\right)
$$

which can also be written using our forward and backward variables as:

$$
\xi_{\mathrm{t}}(\mathrm{i}, \mathrm{j})=\frac{\alpha_{t}(i) a_{i j} b_{j}\left(O_{t+1}\right) \beta_{t}(j)}{P(O \mid \lambda)}
$$

We can re-estimate our model parameters by essentially using Maximum Likelihood Estimates. We can estimate a couple of transitions values as

$$
\begin{aligned}
& \sum_{t=1}^{T-1} \gamma_{t}(i)=\text { Expected transitions from } \mathrm{S}_{\mathrm{i}}\left(\text { expected occurrences of } \mathrm{S}_{\mathrm{i}}\right) \\
& \sum_{t=1}^{T-1} \xi_{\mathrm{t}}(\mathrm{i}, \mathrm{j})=\text { expected transitions from } \mathrm{S}_{\mathrm{i}} \text { to } \mathrm{S}_{\mathrm{j}}
\end{aligned}
$$

Using these estimations we can estimate parameter values of $\lambda$ : 


$$
\begin{aligned}
& \bar{\pi}_{i}=\gamma_{1}(\mathrm{i}) \\
& \overline{a_{i j}}=\text { Expected transitions from } \mathrm{S}_{\mathrm{i}} \text { to } \mathrm{S}_{\mathrm{j}} / \text { expected transitions from } \mathrm{S}_{\mathrm{i}}
\end{aligned}
$$

$$
\overline{b_{i}(k)}=\frac{\sum_{t=1}^{T, t_{-} o_{t}=k}}{\sum_{t=1}^{T} \gamma_{t}(j)}=\text { expected number of times in state } \mathrm{j} \text { and observing } \mathrm{K} / \text { times in } \mathrm{j}
$$

If we view this as an adaptation of the Expectation Maximization algorithm, we can say that computing the expected values is the E step and assigning the model parameter values to their new estimates is the $\mathrm{M}$ step. Iterating over the $\mathrm{E}$ and $\mathrm{M}$ step guarantees a convergence to local optimum parameter values for the HMM model.

The HMMs can vary in topology, or shape of the graph, for example, they can be ergodic, which means there exist non-zero transitions probabilities between all states. They can also form a linear-chain, also known as Bakis models, that are convenient for modeling a signal whose properties change over time. In this kind of model, once a state has been transitioned out of, it cannot be returned to.

Another characteristic that can be changed between models is the form of the observation distributions. Up to this point, we have used discrete state probabilities, but one can also use continues distributions, for example, a Gaussian Mixture Model.

The HMM can be use used in Named Entity Recognition by structuring words in a document as observations and underlying states modeled to include states such as 
"problem", "treatment", "test," and "other." Given labeled training data where the word/states are labeled given the above states, one can train an HMM to find the most likely state sequence given the sequence of words, and in so doing, identify words that fall into the four categories. Additionally, HMMs have been used for Part of Speech (POS) tagging [12]. The Apache OpenNLP library [13] implements HMMs to perform various tasks, such as POS tagging and sentence boundary detection.

\section{Maximum Entropy Markov Models}

Hidden Markov Models allow us to expand the capabilities of the models from stochastic state transition to a twice stochastic model, with stochastic transitions and stochastic state-dependent observations, greatly increasing the expressive power. However, the data we are concerned with has much more information available than can be incorporated into an HMM. We are limited to just the observation (word) and the state (part of speech, for example). There's no way to include information like "this word is capitalized," or "this is the second word in the sentence."

In addition to the limited expressiveness of the feature set, HMMs are modeling a joint distribution between the state transitions $\mathrm{P}\left(\mathrm{s}^{\prime} \mid \mathrm{s}\right)$ and observation $\mathrm{P}\left(\mathrm{o} \mid \mathrm{s}^{\prime}\right)$. In fact, for the problems we approach in text processing, we are given the observation sequence and want to predict the state sequence. So it is more appropriate to our problem to condition 
the state transitions on the current state, and the next observation. There is no need to directly model the probability of the observation sequence.

Enter Maximum Entropy Markov Models [8]. MEMMs replace the joint distribution model with conditional functions $\mathrm{P}\left(\mathrm{s} \mid \mathrm{s}^{\prime}, \mathrm{o}\right)$. One can think of these functions as representing state transitions, instead of states and observations. How this is carried out in practice is by training $|\mathrm{S}|$ Maximum Entropy [9] models, one for each state, that serve to predict the probability of transitioning into the state given a feature set. At each time step, all of the classifiers compute the probability of transitioning into their state given the current values of the feature set. The state with the highest probability is then transitioned into.

Because we have added this new layer of classification functions, we have gained the freedom to expand the feature set and we are no longer limited to just states and observations. Features can include elements like "this is the last word of the sentence" or "there is a hyphen here." Doing this greatly expands the power of the model to take advantage of the available information.

The maximum entropy functions are an exponential model of the form

$$
\mathrm{P}_{s^{\prime}}(s \mid o)=\frac{1}{Z\left(o, s^{\prime}\right)} \exp \left(\sum_{a} \lambda_{a} f_{a}(o, s)\right)
$$

Where $\mathrm{f}$ is a set of features and $\lambda$ is a set of weights to be learned. $\mathrm{Z}\left(\mathrm{o}, \mathrm{s}^{\prime}\right)$ is a normalizing value to ensure the distributions sum to 1 . 
One would imagine that given such alterations to the standard HMM model, you would be required to devise new training and execution algorithms. If we look back to the three basic questions of HMMs, the same questions apply to MEMMs. Luckily, the solutions for HMMs are very similar to the solutions for MEMMs. We used the recursively defined forward variable in the dynamic programming solutions to the first problem. With MEMMs, we make a slight alteration to the forward variable to include the new maximum entropy function defined above,

$$
\alpha_{t+1}(s)=\sum_{s^{\prime} \in S} \alpha_{t}\left(s^{\prime}\right) \cdot \mathrm{P}_{s^{\prime}}\left(s \mid o_{t+1}\right)
$$

With this, the forward-backward algorithm and Viterbi algorithm are very similar to HMMs.

Again, for solving the third basic problem, the solution for MEMMs is very similar to HMMs. The paper [8] gives the approach the name "Generalized Iterative Scaling" but it is essentially a modification of Expectation Maximization.

Adapting HMMs to better suit the problems encountered in Text Processing and Information Extraction significantly boosts the performance of the models on tasks such as segmenting FAQ text into the Questions and the Answers. MEMMs are recommended for POS tagging in [14] for its ability to incorporate more information into the tagging decision. 


\section{Conditional Random Fields}

So far in our march towards better sequence labeling algorithms, we have started with stochastic graphical models, Markov Chains, and expanded them in include stochastic observations which better suit our problem of modeling an underlying state sequence from a sequence of observations, giving us Hidden Markov Models. However, a peculiar problem arises in the sequential modeling of state transitions called Label Bias.

The Label Bias problem says that a model will be biased towards state sequences with lower entropy, meaning state sequences that have encountered fewer branches during transitions. The reason behind the problem is that while computing state sequences, the probability mass of a given state in the sequence is distributed among possible transition states, but if there is only one possible transition, the entire probability mass will be transferred to the state, instead of divided between several states. This is because the probability of the transition is 1 . When there is only a single possible transition, the observation at that state is effectively ignored, because regardless of what the observation was, there is only one possible transition.

To address this problem and others, Conditional Random Fields [10] were developed. To replace the per-state transition functions in MEMMs, CRFs use a joint distribution model over the entire sequence of states given a particular sequence of observations. Doing so alleviates the directed graph sequence nature of MEMMs and there is no longer a Label Bias problem. 
As with MEMMs, as set of feature functions is defined, but unlike MEMMs, these functions are now conditioned on the entire state sequence, not just an individual state. One can define a set of CRF features that mimic exactly an MEMM. This makes MEMM's a special case of the more general CRF model. The general difference between the two models can be observed in the diagram:
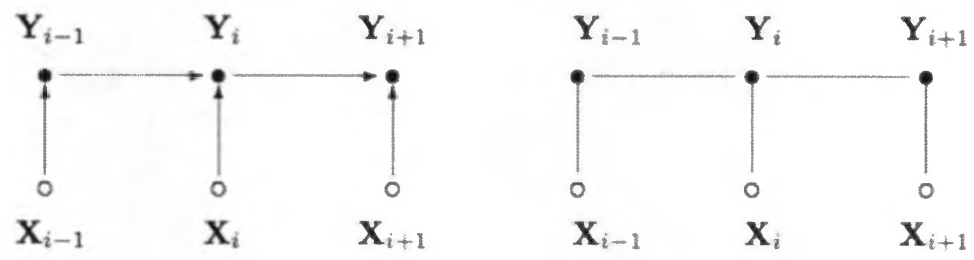

The MEMM on the left shows that a state $Y_{i}$ is conditioned on $Y_{i-1}$ and $X_{i}$ where the CRF on the right is a joint model conditioned on the state sequence $\mathrm{Y}$ and observation sequence X.

An additional benefit of the CRF model over MEMMs is that the training functions converge to a global optimum, whereas MEMMs converge to a local optimum, however, the training procedure takes much longer than MEMMs.

Because the feature set of CRFs is conditioned on the entire state sequence, it allows us to define features that represent long range dependencies spanning the entire state sequence. An empirical benefit of this approach is that in the context of the Named Entity Recognition problem, CRFs tend to outperform HMMs and MEMMs while identifying entities that are several words long. 
The BANNER [14] biomedical named entity recognition system is built on top of the CRF implementation distributed with the MALLET machine learning toolkit [15]. We performed empirical evaluations of four NER systems, two of which employ Markov models in the underlying implementation before a "dictionary lookup" phase. These two systems are MetaMap [16] and cTAKES [17]. The fourth system, MGrep [18], has an undisclosed underlying mechanism. The results from our evaluations showed that the CRF-based BANNER system had the best performance on our evaluation corpus. While many implementation factors are at play that affect individual system performance, we believe that BANNER's superior performance is at least partially a result of its application of the CRF model.

\section{References}

[1] Breiman, Leo. "Random forests." Machine learning 45.1 (2001): 5-32.

[2] Hearst, Marti A., et al. "Support vector machines." Intelligent Systems and their Applications, IEEE 13.4 (1998): 18-28.

[3] Hornik, Kurt, Maxwell Stinchcombe, and Halbert White. "Multilayer feedforward networks are universal approximators." Neural networks 2.5 (1989): 359-366

[4] Bates, Douglas M., and Donald G. Watts. Nonlinear regression: iterative estimation and linear approximations. John Wiley \& Sons, Inc., 1988.

[5] Gardiner, Crispin W. Handbook of stochastic methods. Vol. 4. Berlin: Springer, 1985

[6] Rabiner, Lawrence R., and Biing-Hwang Juang. "An introduction to hidden Markov models." ASSP Magazine, IEEE 3.1 (1986): 4-16.

[7] Moon, Tood K. "The expectation-maximization algorithm." Signal processing magazine, IEEE 13.6 (1996): 47-60.

[8] McCallum, Andrew, Dayne Freitag, and Fernando CN Pereira. "Maximum Entropy Markov Models for Information Extraction and Segmentation." ICML. Vol. 17. 2000.

[9] Berger, Adam L., Vincent J. Della Pietra, and Stephen A. Della Pietra. "A maximum entropy approach to natural language processing." Computational linguistics 22.1 (1996): 39-71

[10] Lafferty, John, Andrew McCallum, and Fernando CN Pereira. "Conditional random fields: Probabilistic models for segmenting and labeling sequence data." (2001).

[11] Wallach, Hanna M. "Topic modeling: beyond bag-of-words." Proceedings of the 23rd international conference on Machine learning. ACM, 2006. 
[12] Collins, Michael. "Discriminative training methods for hidden markov models: Theory and experiments with perceptron algorithms." Proceedings of the ACL- 02 conference on Empirical methods in natural language processing-Volume 10. Association for Computational Linguistics, 2002.

[13] https://opennlp.apache.org/

[14] http://banner.sourceforge.net/

[15] http://mallet.cs.umass.edu/

[16] Aronson, Alan R., and François-Michel Lang. "An overview of MetaMap: historical perspective and recent advances." Journal of the American Medical Informatics Association 17.3 (2010): 229-236.

[17] Savova, Guergana K., et al. "Mayo clinical Text Analysis and Knowledge Extraction System (cTAKES): architecture, component evaluation and applications." Journal of the American Medical Informatics Association 17.5 (2010): 507-513

[18] Dai, M., N. H. Shah, and W. Xuan. "An efficient solution for mapping free text to ontology terms. AMIA Summit on Translational Bioinformatics." San Francisco CA (2008). 